\title{
Compendium of Instrumentation Frontier Whitepapers on Technologies for Snowmass 2013
}

September 13, 2013

\section{Conveners: M. Demarteau, R. Lipton, H. Nicholson, I. Shipsey}

A. Albayrak-Yetkin, J. Alexander, M. Artuso, R. Ball, M. Battaglia, C. Bebek, J. Beene, Y. Benhammou, E. Bentefour, M. Bergevin, A. Bernstein, B. Bilki, E. Blucher, G. Bolla, D. Bortoletto, N. Bowden, G. Brooijmans, K. Byrum, G. Cancelo, C. Chang, J. Chapman, D. Christian, W. Cooper, J. Corso, C. Da Via, S. Dazeley, P. Debbins, G. Deptuch, S. Dhawan, V. Di Benedetto, S. Dye, J. Estrada, H. Evans, E. Etzion, J. Fast, C. Ferretti, B. Fleming, K. Francis, P. Friedman, H. Frisch, M. Garcia-Sciveres, C. Gatto, G. Geronimo, G. Gilchriese, C. Grant, A. Grillo, E. Grunendahl, P. Gorham, G. Gutierrez, C. Haber, G. Haller, C. Hast, U. Heintz, D. Hitlin, C. Hogan, M. Hohlmann, G. Jennings, M. Johnson, H. Kagan, C. Kenney, R. Khanna, V. Khristenko, F. Krennrich, J. Learned, D. Levin, R. Lipton, T. Liu, D.

Lissauer, D. Lynn, D. MacFarlane, S. Majewski, J. Maricic, P. Marleau, A. Mazzacane, A. Mestvirisvilli, N. Mokhov, M. Moshe, M. Newcomer, M. Narian, K. Nishimura, D. Nygren, E. Oberla, Y. Onel, A. Para, S. Parker, V. Polychronakos, E. Ramberg, B. Rebel, J. Repond, D. Reyna, A. Ronzhin, R. Rusack, A. Ryd, H. Sadrozinski, C. Sanzeni, S. Seidel, A. Seiden, I. Schmidt, A. Shenai, Y. Silver, W. Smith, A. Sonnenschein, D. Southwick, L. Spigel, M. Stanitzki, S. Striganov, D. Su, R. Sumner, R. Svoboda, M. Sweany, R. Tayloe, N. Terentiev, J. Thom, W. Trischuk, M. Vagins, K. Van Bibber, G. Varner, R. Varner, J. Va'vra, H. Von der Lippe, R. Wagner, C. Weaverdyck, H. Wenzel, M. Wetstein, R. Wigmans, D. Winn, A. White, L. Xia, Z. Ye, M. Yeh, T. Yetkin, J. Yoo, J. Yu, B. Zhou, R. Zhu

\section{Contents:}

- Beam Test Facilities

- Sensor Compendium 
- Needs for the Energy Frontier

- Needs for the Intensity Frontier

- Needs for the Cosmic Frontier

- Monolithic Pixel Sensors

- Hybrid Pixel Detector Challenges for next Generation Frontier Experiments at the LHC

- 3D Architecture Pixel Sensors

- Diamond Sensors

- Ultra-fast Silicon Detectors

- Next Generation Strip Detectors for the Energy Frontier

- Next Generation Strip Detectors for the Intensity Frontier

- Low Mass Support and Cooling for Future Collider Tracking Detector

- Development of Large-Area Photosensors for Future HEP Experiments

- Solid State Photodetectors

- Crystal Detectors

- The Large Area Picosecond Photodetector (LAPPD) Project

- Transition Edge Sensors for High Energy Physics

- Instrumentation challenges for Direct Detection of Dark Matter

- 3D Technologies for Large Area Trackers

- 3D Geometry Sensors

- Use of Flat Panel Microchannel Plates in Sampling Calorimeters with Timing

- Combination of Active Edge and 3D Electronics Technologies

- Diamond Sensors

- A Differential Time-of-Flight Technique for Collider Detectors

- Future Developments in Gigasample-per-second Waveform Sampling Application Specific Integrated Circuits

- Opportunities for HEP Technology Evaluation in Smaller-Scale NP Experiments

- Applications of Laser Interferometry in Fundamental Physics Experiments 
- Large arrays of SiPMs for HEP and industry

- Ultra-Low Index and Rad-Hard Total Internal Reflection Claddings for High Numerical Aperture Scintillating, Waveshifting, Cerenkov, or Clear Optical Fibers, Capillaries, and Plates

- Monolithic Pixel Sensors

- Novel Compact Accelerator-Based Neutron and Gamma Sources for Detector Calibration

- The Next Generation of Photo-Detectors for Science in the Cosmic, Intensity and Energy Frontiers

- Secondary Emission Calorimetry: Fast and Radiation-Hard

- Quartz Capillary Cladding Anthracene and Polycyclic Aromatic Hydrocarbon(PAH)-Core Scintillating/WLS Fibers for High Rates and Radiation Damage Resistance

- Micro-Pattern Gas Detectors for Calorimetry 


\section{Beam Test Facilities}

\section{J. Yu (Univ. of Texas at Arlington), C. Hast (SLAC National Lab.) and E. Ramberg (FNAL)}

Physics Driver: All physics in the future requires dramatically improved functionalities to meet the challenges in various experimental conditions. The high luminosity requirements for precision measurements will demand detectors with fast response. The high energy and/or high beam flux environments require radiation hard detectors. The level of precisions demanded by the future physics goals requires high energy and position resolution detectors. These new and significantly improved detectors necessitate considerable R\&D before they can be used in actual experiments in all frontiers. Beam test facilities provide essential test grounds beyond the simulation and bench tests for R\&D crucial for future experiments.

Time Frame: Short, Medium \& Long

Physics Justification: All types of detectors - starting from vertex to tracking, to particle ID and calorimetry to muon detectors - face considerable challenges to meet the needs of the future experiments. For example, precise determination of Higgs properties at the LHC and the Linear Collider require unprecedented energy resolution of calorimeters and radiation hard detectors. Neutrino and rate decay experiments require improved particle identification capabilities and challenges in the recently popular liquid argon TPCs. Searches for dark matter and dark energy in space based experiments require large area, light-weight detectors. All these detectors will have to be studied at beam test facilities to extensively test them and verify their functionalities.

Technical Requirements: Beam test facilities need to meet the anticipated demand for testing detector capabilities thoroughly. They will need to be able to provide various species of particles - electrons, muons, pions, kaons, protons and, if possible, neutral hadrons, including neutrons, with momentum tags. They must be able to provide as wide a range of particle momenta as possible and should provide multiple experimental areas for simultaneous running of beam test experiments. The beam lines must be able to provide sufficiently narrow momentum bites to reduce the systematic uncertainties resulting from particle momentum. The facility must also provide beam line instrumentation for in-situ beam particle identifications, along with a plug-in infrastructure, such as HV supplies, low voltage suppliers, DAQ and trigger systems and computer network. Adjustable beam flux from few $\mathrm{Hz}$ to few $\mathrm{MHz}$ is desirable to meet the requirements for various running conditions.

Current Technical Capabilities: While different facilities have different capabilities, many existing beam test facilities already meet most of the technical requirements above. More sophisticated functionalities related to beam timing trigger signal, neutron tagged beams and finer beam flux adjustments need more improvement. The biggest challenges, though, stems from the fact that these beam test facilities are not the primary missions of the laboratory and thus the operation in many cases are limited.

\section{Key R\&D Directions:}

- Providing neutral hadrons with momentum tag

- Flexible and adjustable beam timing structure 


\title{
Snowmass Whitepaper
}

August 8, 2013

\section{Sensor Compendium}

\author{
Marina Artuso $^{(a)}$ and Abraham Seiden ${ }^{(b)}$
}

(a) Syracuse University

(b) University of California Santa Cruz

\begin{abstract}
Sensors play a key role in detecting both charged particles and photons for all three frontiers in Particle Physics. The signals from an individual sensor that can be used include ionization deposited, phonons created, or light emitted from excitations of the material. The individual sensors are then typically arrayed for detection of individual particles or groups of particles. Mounting of new, ever higher performance experiments, often depend on advances in sensors in a range of performance characteristics. These performance metrics can include position resolution for passing particles, time resolution on particles impacting the sensor, and overall rate capabilities. In addition the feasible detector area and cost frequently provides a limit to what can be built and therefore is often another area where improvements are important. Finally, radiation tolerance is becoming a requirement in a broad array of devices. We present a status report on a broad category of sensors, including challenges for the future and work in progress to solve those challenges.
\end{abstract}




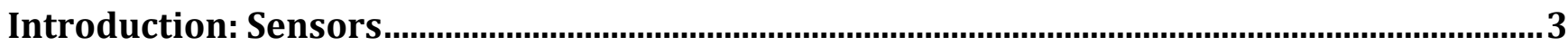

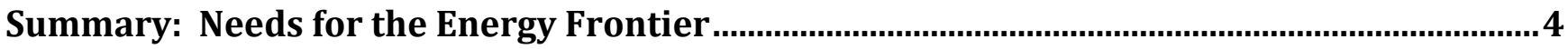

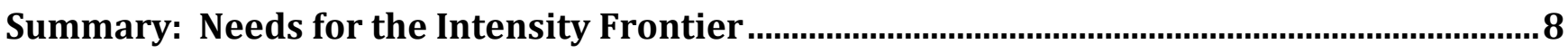

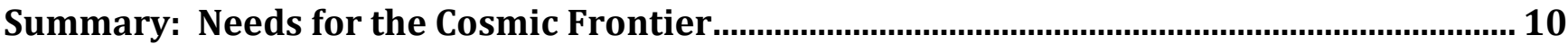

Monolithic Pixel Sensors .......................................................................................................... 12

Hybrid Pixel Detector Challenges for next Generation Frontier Experiments at the LHC... 19

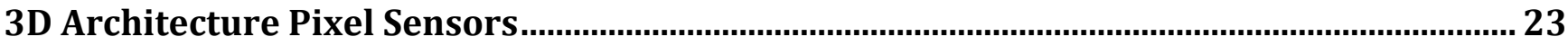

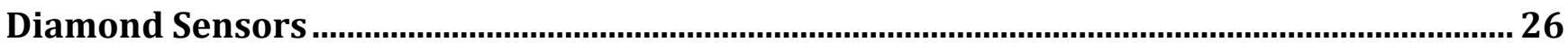

Ultra-fast Silicon Detectors (4D-UFSD) ……............................................................................ 31

Next Generation Strip Detectors for the Energy Frontier...................................................... 37

Next Generation Strip Detectors for the Intensity Frontier ........................................................44

Low Mass Support and Cooling for Future Collider Tracking Detectors .................................. 46

Development of Large-Area Photosensors for Future HEP Experiments............................... 52

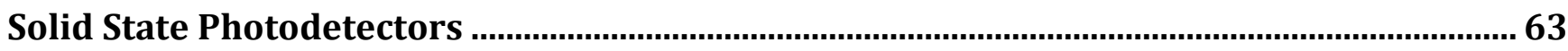

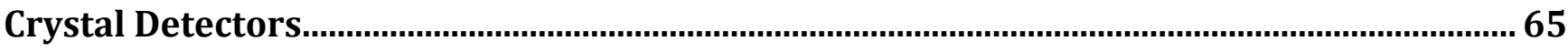

The Large Area Picosecond Photodetector (LAPPD) Project............................................................ 69

Transition Edge Sensors for High Energy Physics ................................................................... 73

Instrumentation challenges for Direct Detection of Dark Matter............................................... 76 


\section{Introduction: Sensors}

\section{Contribution of Marina Artuso (Syracuse) and Abe Seiden (UC Santa Cruz)}

Sensors play a key role in detecting both charged particles and photons for all three frontiers in Particle Physics. The signals from an individual sensor that can be used include ionization deposited, phonons created, or light emitted from excitations of the material. The individual sensors are then typically arrayed for detection of individual particles or groups of particles.

Mounting of new, ever higher performance experiments, often depend on advances in sensors in a range of performance characteristics. These performance metrics can include position resolution for passing particles, time resolution on particles impacting the sensor, and overall rate capabilities. In addition the feasible detector area and cost frequently provides a limit to what can be built and therefore is often another area where improvements are important. Finally, radiation tolerance is becoming a requirement in a broad array of devices. The system performance also depends crucially on the electronics chain used for readout. In many cases issues such as mechanical support, cooling, cabling and voltage distribution are critical to good performance. Some specific findings and recommendations are given below.

Findings and Recommendations: Summary

1. Low mass, high radiation requirements imply thinner detectors with lower signal levels, thus implying low noise electronics, with zero-suppressed readout (because of the high channel count) implemented with discriminators featuring low and stable thresholds. This will require significant work on electronics design as well as use of the latest chip technologies. A large number of sensor choices are under investigation, which have different advantages in terms of radiation hardness, low mass, and high speed targeted to the different emphases of the three frontiers.

2. Fine pitch, connector-less, reliable electrical connections are key to achieve the low mass-fine pitch detector systems that are planned for future applications. This is an area in which much more work could be done.

3. The goal of minimizing the detector mass implies an integrated system design incorporating cooling and power distribution as primary design considerations. A coordinated effort to develop new materials, establish a data base of their properties, and implement the standardization of their quality assurance certification is key to multiple science frontiers and has a dramatic spin-off potential.

4. Cosmic frontier applications span a very large spectrum of technologies and techniques but often have in common the requirement for low and well known background levels. A community wide approach to radiopure materials and assay is likely to be very beneficial. Some of the novel sensors being developed are Transition Edge Sensors and Microwave Kinetic Induction Detectors, thick CCDs, and large Germanium detectors. Low activity photomultipliers are very important for experiments using noble liquid detectors.

5. A number of other photon detection and timing devices are very important for progress at several of the frontiers. This includes solid state photodetectors, and a number of devices with significantly improved timing capabilities.

6. Crystal material suitable for large scale calorimeters, capable of withstanding high fluences, and featuring faster time response fulfill a central role in intensity and energy frontier applications. 


\section{Summary: Needs for the Energy Frontier}

\section{Contribution of Daniela Bortoletto (Purdue University)}

A next application of sensors for the Energy Frontier can be expected to be for the vertex detectors and trackers for the upgrades of CMS and ATLAS. The increased luminosity of the LHC poses severe challenges. Since the luminosity of the LHC will increase, the number of interactions per crossing will also rise from 20 to at least 140 and possibly 200 every $25 \mathrm{~ns}$. In order to keep the occupancy below $1 \%$, we must reduce each cell size, and increase the granularity, and therefore the number of readout channels. This trend requires the development of smaller detector elements and it introduces challenges since it necessitates many channels equipped with fast and thus power dissipating frontend electronics. Especially in the layers closest to the interaction region, the next generation of detectors must also reach high rate capabilities using integrated circuits and materials with increased tolerance to radiation dose and single event upsets.

Future trackers must function in this environment while maintaining or improving their performance. Pixel detectors are essential to seed tracking, select the primary vertex of hardscatter events of interest, and mitigate the effect of the pileup. To fully identify many physics signatures, trackers and vertex detectors must provide excellent position and momentum measurements for high- p $_{\mathrm{T}}$ leptons. They also have to establish that leptons are in fact isolated. The material in the tracker and the vertex detectors affects the efficiency and resolution to detect isolated electrons, due to bremsstrahlung and nuclear interactions. Therefore it is critical to reduce this degradation by diminishing the material. The performance of b-tagging algorithms based on displaced vertices also requires controlling the tails in the impact parameter measurement due to errors in pattern recognition and hadronic interactions within the active volume of the vertex detector. Therefore the upgrades strive to achieve a larger channel count while maintaining a careful control on the material inside the tracking. This has profound implication on the mechanical design of the support structure, the powering scheme, and the cooling.

For pixel vertex detectors we must develop sensors capable to survive doses of about $2 \times 10^{16}$ $\mathrm{neq} / \mathrm{cm}^{2}$ or provide easy replacement of the innermost pixel layers. The pixel readout chip must handle rates of about $1 \mathrm{GHz} / \mathrm{cm}^{2}$. This requires smaller, faster pixel chips, with increased functionality. Both CMS and ATLAS are focusing on the development of new electronics using the $65 \mathrm{~nm}$ CMOS technology. Thin planar sensors [1] operated at high voltage (about 700V for 75 $\mu \mathrm{m}$ ) show $60 \%$ charge collection efficiency after $10^{16} \mathrm{neq} / \mathrm{cm}^{2}$. Non-planar 3D sensors have been shown to be radiation hard up to doses of $5 \times 10^{15} \mathrm{neq} / \mathrm{cm}^{2}$ and they will used in a new inner pixel layer, the ATLAS IBL[2]. R\&D should occur to further increase their radiation hardness. The reduced bias voltage needed to operate 3D sensors is also of great interest since it could allow lower mass cooling systems. Diamond remains an attractive option, even if concerns continue to exist regarding material availability and decreased signal collection after irradiation due to 
trapped charge forming a polizarization field. Because of the smaller charge collected in diamond with respect to silicon, to take full advantage of diamond detectors new very low threshold readout chips must be developed. The DIAPIX development [3] is going in this direction and aims at using 3D electronics to achieve a threshold of about $1000 \mathrm{e}-$. For hybrid pixel detectors the smaller pixels are likely to have an area of about $2500 \mu \mathrm{m}^{2}$. This enables increased resolution and better two-track separation. The pixel size and the material could be further reduced using monolithic pixels. However, the development of radiation hard CMOS pixels for hadron colliders is at a very early stage.

The next generation trackers will cover an area of about $200 \mathrm{~m}^{2}$, similar to the one installed in the current CMS detector. Also for the outer layers of the tracker, the increased luminosity requires the development of smaller size devices such as macro-pixel, strixels, or short strips, that will also augment the achievable resolution. Pitch reduction will also improve the tracker performance at high $\mathrm{p}_{\text {T. }}$ The higher tracker granularity, especially the decrease in strip length down to centimetres or even millimetres, poses new challenges to sensor channel connectivity to the readout [4]. This can already be seen for example in the progress of the module design of the silicon micro-strips for the ATLAS upgraded tracker, where the sensitive strip length shrinks from $12 \mathrm{~cm}$ (obtained by daisy chaining two sensors) to $2.4 \mathrm{~cm}$, forcing the placement of the hybrids directly over the surface of the sensor. This example of a connectivity problem is solved using "traditional" methods, but the anticipated higher channel density would certainly benefit from novel solutions such as multi-metal routing, dielectric-metal interposers, combined use of bump and wire bonding, through silicon via (TSV) structures on electronics and/or sensors. Double metal structures are in use since LEP and the Tevatron and also today in the LHCb VELO detector. This last example shows a complex routing of inner short strips to the outer edge of the sensors by means of second metal lines. This routing can lead to charge losses or an increase in noise. For short, such as strips (strixels) in the range of millimetres, bump bonding seems to be advantageous but a more elaborate routing to dedicated bonding areas may be beneficial to avoid covering the whole sensor with chips. This would reduce the overall mass and cost of the system. $\mathrm{R} \& \mathrm{D}$ is needed to better understand the performance and limitation of routing in multi metal layers, in-between channels using the first metal, and using deposited thin dielectric and metal layers (e.g. Multi-Chip Module deposited, MCM-D). MCM-D is a technology that can be applied to silicon strip modules and promises advantages in terms of integration complexity and material budget. It allows integrating the front-end hybrid, pitch adaptor and wire bonds on the silicon sensor by depositing dielectric and metal layers on silicon, where traces and vias are etched with high resolution to produce a PCB like structure. Initial positive results have been obtained with MCM-D layers but further work for improving the density of lines and contacts needs to be performed.

One of the key issues for strip sensors deployed in a future tracker is to withstand fluences of the order of $2 \times 10^{15} \mathrm{n}_{\text {eq }} \mathrm{cm}^{-2}$. While these specifications are not as stringent as for pixel detectors, $R \& D$ is still necessary in order to find cost effective radiation hard sensors. Dedicated R\&D has 
been conducted both by ATLAS and CMS. The CERN-RD50 collaboration has also been instrumental in the study of the radiation issues for the HL-LHC. The measurements that have been conducted indicate that the n-on-p sensors match the requirements of the HL-LHC. Some advantages have been identified using oxygen enriched MCz material. CMS is also starting to evaluate the potential of deploying sensors fabricated on 8" wafers at Infineon.

An important requirement of future trackers motivated by the luminosity increase is the need for intelligent trackers providing fast momentum information to select events of interest. R\&D is ongoing on several options including reading out the tracker data only in regions of interest, and on detector data reduction by using the correlations between top and bottom sensors necessary to measure the track curvature, measuring the $\mathrm{p}_{\mathrm{T}}$ using associative memories.

The importance of reducing the material is especially critical for $\mathrm{e}^{+} \mathrm{e}^{-}$colliders but, as stated above, it is also important for the HL-LHC. R\&D for the ILC and CLIC pixel detectors has focused on using CMOS or SOI (Silicon on Insulator) technologies that combine particle detection and read-out electronics in the same device [5]. The adoption of these commercial technologies is especially interesting since it could also bring significant cost savings. The $90 \mathrm{~nm}$ and $65 \mathrm{~nm}$ CMOS technology are currently being evaluated as a promising solution to the integration of highdensity circuitry and high-speed functionalities in a small pixel. Present CMOS sensors do not have a deep depletion volume and are using only the shallow depletion depths produced by the junction built in voltage as sensor volume. For hadron collider applications the most significant issues to be solved are radiation hardness and the possibility to deplete the sensitive layer. Highvoltage CMOS pixels are currently being pursued for the LHC upgrades since the strong electric field in the fully depleted volume allows the fast charge collection needed for radiation tolerance. It also reduces the cluster size and improves two-track separation. The SOI process developed by KEK with Lapis Semiconductor using a high-resistivity handle wafer ensures large signals, low collection electrode capacitance, and low-power and therefore could provide radiation tolerant devices.

Strip or mini-strip sensors have larger cells size than typical pixel sensors, and thus higher capacitance and noise. Therefore sensors must collect a large signal from a significant depleted volume. To exploit the SOI or CMOS potential in these systems, novel design concepts have to be developed to combine small electronic feature size with high depletion voltage for the sensor bulk, without impacting on the performance of the electronics processed above the sensitive volume. R\&D must be directed towards the application of these technologies to strip or mini-strip sensors to increase the efficiency of future trackers. Since a mini-strip cell size is relatively large this could yield the possibility of implementing additional intelligence in the available area such as large buffer sizes, sparsification, ADC, and trigger logic. This approach could help to reduce the material of tracker systems and solve the complex routing for the strixel/macro-pixel geometry. $R \& D$ in this area would be beneficial for the outer tracker and the inner pixel region of the LHC detectors and is relevant for future $\mathrm{e}^{+} \mathrm{e}^{-}$colliders. 


\section{References}

[1] A.Macchiolo et al, arXiv : 1210.7933

[2] The ATLAS IBL Collaboration, "Prototype ATLAS IBL Modules using the FE-I4A Front-End Readout Chip", submitted to JINST, http:// arxiv.org /abs/1209.1906.

[3] G. Chiodini,"Radiation detectors based on synthetic diamond", Incontri di Fisica delle Alte Energie (IFAE2012). Università di Ferrara, April 11-13, 2012.

[4] G. Casse and F. Hartmann, Trends and perspectives for large area tracking systems, contribution to the European Strategy group.

[5] M. Battaglia et al., Nucl. Instrum. and Meth. A716 (2013), 29. 


\section{Summary: Needs for the Intensity Frontier \\ Contribution of Marina Artuso (Syracuse), Matt Wetstein (UChicago/ANL), M. C. Sanchez (Iowa State/ANL)}

Experiments planned for the "intensity frontier" encompass a broad spectrum of studies and technologies. Experiments optimized for neutrino detection will explore the neutrino mass hierarchy, search for $\mathrm{CP}$ violation and non-standard interactions, and elucidate the nature of neutrinos (Dirac or Majorana). In additions, large scale neutrino experiments will increase sensitivity to proton decay. Extremely rare muon and tau decay experiments will search for violation of charged lepton quantum numbers. Rare and CP-violating decays of bottom, charm, and strange particles will allow to probe new physics at very high mass scale and will be complementary to direct searches for new phenomena at the high energy frontier in our effort to unravel the mass scale and nature of new physics.

A key element in detector systems designed to study heavy quark and charged lepton decays is precision tracking and vertexing that relies on the most advanced Si based micropattern detectors. The subsystem in closer proximity to the interaction region involve pixel detectors, either monolithic (DEPFET) [1] or hybrid [2]. These subsystems are complemented by silicon microstrip detectors, where the sensor is generally based on a proven technology, but where the "material challenge" to minimize the non-active material crossed by the charged particles is of utmost importance.

Requirements on the front end electronics processing information from Si devices for heavy quark experiments at the intensity frontier shows a general trend towards faster readout and synergy with the energy frontier. For example, the BelleII readout electronics for the strip detector is based on the asic developed for CMS. LHCb presents an interesting evolution of this concept, with fast analog readout and a processing block that allows to push the data out of the detector in real time, and thus allows for a purely software based trigger selection.

The reconstruction and identification of photons, electrons and neutral pions is generally based on Ring Imaging Cherenkov detectors, which require R\&D on faster photon detectors. An alternative based on picosecond time of flight technologies is gaining a lot of attention and may be a very attractive solution for $\mathrm{K}, \mathrm{p}$, and $\pi$ identification in certain momentum ranges.

Photosensors provide essential functionality for HEP experiments in a wide variety of contexts. Increasingly, the demands of HEP experiments are pushing beyond standard capabilities of conventional phototubes. Photosensors are typically used in three broad categories of detector systems: (1) Large water Cherenkov tanks for full event reconstruction in neutrino and proton decay experiments, (2) Ring Imaging Cherenkov (RICH) and Direct Internally Reflected Cherenkov (DIRC) detectors used for particle identification in heavy flavor collider experiments, (3) and simple time-of-flight(TOF) systems used for vertex reconstruction and time-of-flight 
mass spectroscopy in colliders. The demands on these photosensors vary widely from one experiment to the next, but can be divided between the two main types of operational conditions: 1) Low-rate environments such as neutrino, double beta decay, and proton decay experiments. Under these conditions low cost coverage, improved resolution for event reconstruction, and single photon counting capabilities are most important for background rejection, signal selection efficiency, tracking, and momentum resolution. 2) High-rate environments typical of collider based experiments. Here photodetectors need to be robust over years of operation under intense radiation, and capable of handling high event rates per unit area per unit time without saturating. Readout electronics must be designed to handle the high rates and track multiplicities.

Common to all experimental categories is the need to instrument large areas economically and the need for improvements in the combined timing and imaging resolutions.

\section{REFERENCES}

[1] T. Abe et al., Belle II Technical design report, arxiv:1011.0352 (2011)

[2] R. Aaij et al., Letter of Intent for the LHCb Upgrade, CERN-LHCC-2011-001 (2011). 


\section{Summary: Needs for the Cosmic Frontier Contribution of James Fast (PNNL)}

Experiments planned for the "cosmic frontier" span a tremendously vast spectrum of technologies and techniques from low-background experiments deep underground to vast airshower detectors, to telescope and satellite-based survey instruments imaging the cosmos across a broad range of the electromagnetic spectrum. The primary aims of this frontier are the discovery and characterization of dark matter and elucidation of the nature of dark energy. In addition, experiments are probing the highest energy particles and photons in the universe.

The dark matter "generation 2" experiments are currently in the final phase of R\&D with a downselect expected this winter to a set of 2-3 technologies to be fully developed for the next decade of searches. While there is a theoretical bias towards higher masses (several 10's or hundreds of $\mathrm{GeV}$ ), there continue to be tantalizing excesses of events in several experiments that are consistent with a low-mass Weakly Interaction Massive Particle (WIMP) candidate. Since the nature of the dark matter remains a mystery, it is critical that the suite of experiments continues to probe the full spectrum of possible interactions - axions, spin-dependent and spinindependent couplings. Low radiometric background materials and assay techniques to verify that the materials used meet background goals is a common challenge across nearly all of these experiments. While the background requirements are not as strict as those for neutrinoless double-beta decay searches, they are nonetheless quite difficult to achieve. A community-wide approach to radiopure materials and assay is likely to be required as the generation 2 experiments are scaled up and generation 3 experiment concepts are developed.

Dark matter searches use a variety of approaches that utilize all three potential signals from a detector material - phonons, ionization and scintillation - in general trying to exploit two of the three to provide discrimination between WIMP recoil events and ionization events. Hence the sensor research program spans high-fidelity ionization spectrometers, high-performance phonon detectors such as the Transition Edge Sensor (TES) coupled to Superconducting Quantum Interference Devices (SQUIDs), and low-background PMTs operable at cryogenic temperatures for scintillation detection in liquid noble gas detectors. In addition, novel new approaches such as thick CCDs with extremely low thresholds are being pursued to extend the experimental reach to much lower WIMP masses.

Experiments observing the cosmic microwave background (CMB) are focusing R\&D efforts on cryogenic Transition Edge Sensors (TES) and Microwave Kinetic Induction Detectors (MKID) that rely on superconducting phase transitions to measure minute temperature rises due to

interactions of the CMB photons with the sensors. Scaling up to large arrays is a significant challenge for next-generation experiments. 
Finally, there are the large air-shower arrays that will benefit from low-cost, large area photosensors that are under development for many applications across all of the high-energy physics frontiers. 


\section{Monolithic Pixel Sensors}

\section{Contribution of Marco Battaglia (UC Santa Cruz)}

The standard figure of merit for vertex tracking accuracy in collider experiments is the accuracy on the impact parameter, $\sigma_{I P}$, defined as the distance of closest approach of the particle track to the colliding beam position. This can be parametrised as: $\sigma_{\mathrm{IP}}=\mathrm{a}+\mathrm{b} / \mathrm{p} \sin ^{\mathrm{k}} \theta$, where $\mathrm{p}$ is the particle momentum, $\theta$ the track polar angle and $\mathrm{k}=3 / 2$ for the $\mathrm{R}-\phi$ and $5 / 2$ for the $\mathrm{z}$ projection. The identification of hadronic jets originating from heavy quarks is best achieved by a topological reconstruction of the displaced vertex structure and the kinematics associated to their decays. The ability in reconstructing these sequences of primary, secondary and tertiary vertices depends on the impact parameter resolution. This is related to the single point resolution of the sensors and their geometry. The figure of merit for the resolution is the distribution of the impact parameter values for charged particles originating from $b$ hadron decays. It is useful to recall that the impact parameter does not depend, to a first approximation, on the $b$ hadron energy, since the angle of production of the track in the lab frame is proportional to $(\beta \gamma)^{-1}$, which compensates the $\beta \gamma$ term in the $b$ hadron decay length. The average impact parameter value is $\sim 220 \mu \mathrm{m}$, with a $0.15(0.24)$ fraction of tracks having impact parameter below 15 (35) $\mu \mathrm{m}$. The asymptotic resolution that appears in $\sigma_{I P}$ is typically proportional to the sensor point resolution, the tracking lever arm and, to a lesser extent, the radial position, $\mathrm{R}$, of the first layer. The multiple scattering coefficient, b, scales with sqrt(d) $R / p$, where $d$ is essentially the material thickness of the beam pipe and the first layer. The tracking lever arm is constrained by detector volume, number of electronics channels and cost. The minimum detector radius is typically defined by radiation or particle density conditions from particle fluence and beam-induced backgrounds and it is accelerator dependant. This was $63 \mathrm{~mm}$ at LEP and $25 \mathrm{~mm}$ at SLC, is $30-50 \mathrm{~mm}$ at the LHC, with plans to further reduce it, and should be $15-30 \mathrm{~mm}$ at future lepton colliders.

The two main drivers for the sensor pixel pitch, $\mathrm{P}$, are single point resolution and two-track separation, both scaling as $\mathrm{P}$, and local occupancy, scaling as the pixel area (i.e. proportional to $\mathrm{P}^{2}$ for square pixels) times the readout time. As a result of this, starting with the current LHC detectors, fast time-stamping, required by occupancy, has become part of the overall optimization and the space-time granularity has to be considered as the appropriate parameter space for characterizing any future vertex detectors.

Monolithic pixel sensors, where the Si sensitive volume and the charge sensing and possibly part of the readout circuitry are implanted in the same Si wafer, have emerged at the end of the '90s in the framework of the R\&D towards a future linear collider as the way forward towards thin $\mathrm{Si}$ sensors with very high granularity and small pixel capacitance, well beyond the technological capabilities for the hybrid pixel sensors then under development for application in the LHC experiments. This development was made possible by the progress in deep sub-micron CMOS processes and the availability of commercial processes with thick epitaxial layers aimed at 
sensors for optical imaging and consumer digital cameras, where CMOS image sensors have overtaken CCDs in terms of market share.

Monolithic pixels offer less mass than hybrid pixel detectors, lower pixel capacitance (and thus noise) and a single chip solution without bump bonding [1]. Combining particle detection and (at least part of) front-end electronics (FEE) in the same device is a prominent advantage for monolithic CMOS pixels, but this may also turn into a limitation since their manufacturing relies on industrial processes optimized for commercial products, which may depart substantially from those needed for charged particle detection. However, CMOS industry is evolving in a direction which allows CMOS pixels to progressively approach their real potential. Developments towards future vertex detector systems rely on the advances in the CMOS technology to cope with the demanding requirements of future collider experiments. To this purpose, many R\&D paths are being pursued in the vertex detector community, with the US playing an important, although not yet a leading, role. The shrinking of the feature size of CMOS transistors is the most apparent outcome of the evolution of microelectronics. This is being exploited in the design of new pixels where advanced functions can be performed in the circuitry implemented in each pixel to provide the required signal-to-noise ratio and to handle the high data rate. These functions include amplification, filtering, calibration, discriminator threshold adjustment, data sparsification and time stamping.

CMOS sensors for particle tracking were initially designed following the classical guidelines of the NMOS-only 3-transistor cell of image sensors to collect the charge generated in an almost fieldfree epitaxial layer. Now, thanks to CMOS scaling, more functions can be included in the pixel cell, including correlated double sampling and hit binary information from a discriminator. This leads itself to achieve zero suppression at the pixel level, which is a highly desirable feature given the large number of channels foreseen and the fast readout of the pixel matrix. New strategies are also being devised in terms of the needed readout architecture, introducing a higher degree of parallelism to speed up the transfer of information from pixels to the chip periphery. Another way to comply with a high hit rate is to adopt the solutions developed for hybrid pixel sensors. This requires that the classical continuous-time analog signal processing chain (charge-sensitive preamplifier, shaping filter) is integrated in the pixel cell, together with the digital blocks needed for a high-speed sparsified readout of the matrix [2].

The implementation of complex circuitry in the pixel cell requires a technique to insulate the transistors from the collection volume, to avoid parasitic charge collection and cross talk. This can be done using large deep $\mathrm{N}$-well electrodes as sensing elements or by profiting from the thin oxide layer of the silicon-on-insulator (SOI) technology $[3,4]$ insulating the CMOS electronics to use full CMOS capabilities, without degradation of the charge collection efficiency.

These technologies make it possible to implement advanced readout architectures, that can be tailored to different applications. However, special care needs to be taken to avoid analog-digital coupling. In the last few years, successful results have been reported for the 130nm CMOS technology node [5] and the $200 \mathrm{~nm}$ SOI process [4]. Recently, the $180 \mathrm{~nm}$ CMOS process featuring 
a nearly $20 \mu \mathrm{m}$ thick epitaxial layer with resistivity exceeding $1 \mathrm{k} \Omega \mathrm{cm}, 6$ to 7 metallization layers and additional P-wells offers the possibility to use both types of transistors inside the pixels, thus allowing for a substantial increase of the in-pixel micro-circuit complexity [6]. The 90nm and $65 \mathrm{~nm}$ CMOS technology nodes are currently being evaluated as a promising solution to the integration of high density circuitry and high speed functionalities in a small pixel. High-voltage CMOS pixels [7] were pioneered several years ago with a larger feature size process [8] and their development is currently pursued on smaller feature size in the perspective of tracker upgrades at the LHC.

Besides device scaling, advanced CMOS technologies bring along other desirable features that pixel R\&D projects are presently exploring. Metal interconnections are isolated by low-k dielectrics to reduce parasitic capacitance and time constants of transmission lines. This may be exploited by minimizing the electronic functions in the pixel cell and by connecting each pixel to signal processing blocks in the chip periphery. Thanks to the very high integration density of the CMOS process, this peripheral readout strategy may require just a small silicon area outside the pixel matrix itself. New strategies are also being devised in terms of the needed readout architecture, introducing a higher degree of parallelism to speed up the transfer of information from pixels to the chip periphery.

Monolithic CMOS and DEPFET technologies offer us sensors with thin active layers, pixel cells of $\mathrm{O}(10 \times 10) \mu \mathrm{m}^{2}$ in size with a typical capacitance of $\mathrm{O}(1) \mathrm{fF}$ and 10-40 e- ENC of noise and significant data processing capabilities implemented in-pixel or on-chip. Costs are low, as long as standard CMOS processes are used, and multi-project runs are widely available, also for several specialised processes.

The optimal pixel size for HEP applications is driven by practical considerations such as the readout-out rate and charge diffusion limit, but pixel pitches of $5 \mu \mathrm{m}$ and below are technically feasible and beneficial for specific imaging applications. An active thickness of $15-20 \mu \mathrm{m}$ is sufficient for achieving a detection efficiency $>99 \%$ with CMOS pixels, even at operating temperatures in excess of $40^{\circ} \mathrm{C}$. Back-thinning technologies, such as grinding and chemical etching now commonly available as commercial service, have been successfully applied to obtain 40-50 $\mu$ m-thick CMOS sensors for the STAR HFT at RHIC and in the R\&D for linear colliders and B-factories. Finally, the monolithic pixel technology is gaining ground in applications at the LHC, for example in the ALICE upgrade where the baseline design is based on monolithic pixels to obtain ladders with an overall material budget $0.3-0.4 \% \mathrm{X}_{0}$ / layer and the sensor contributing $\sim 15 \%$. DEPFET sensors can be thinned to less than $100 \mu \mathrm{m}$ in the sensitive region, using wafer bonding technology, retaining a frame which ensures sufficient stiffness of the mechanical module. Thin DEPFETs are currently under development for BELLE-II, where the target thickness is $75 \mu \mathrm{m}$, and first results have already been obtained for prototypes successfully thinned to 50 $\mu \mathrm{m}$. Beyond HEP there is a broad range of applications where monolithic pixel sensors have already been successfully tested, demonstrating superior performances compared to the 
detectors currently in use. They include X-ray imaging and spectroscopy from light sources and FELs to satellite astronomy, biological autoradiography and plasma diagnostics; low energy electron imaging for transmission electron microscopy and accelerator beam monitoring; optical biological imaging and low momentum particle detection in nuclear physics and real-time dose delivery assessment in hadrontherapy [9] .

The use of a moderate- to high-resistivity thin substrate is the latest step in the process development exploited by monolithic pixel sensors. The depleted sensitive layer enhances both the amount of collected charge and the collection time also reducing the charge carriers spread, compared to the almost field-free epitaxial layer of the standard CMOS active pixel sensors, without compromising the sensor competitive thickness. Given that the reverse bias voltage, $V_{d}$, required to deplete a given thickness $d$ scales as $d^{2} / \rho$, where $\rho$ is the Si resistivity, a modest $V_{d}$ of $\mathrm{O}(10 \mathrm{~V})$ is sufficient to fully deplete a thin layer of high resistivity Si. Operating the detector fully depleted has advantages both in terms of signal charge and of collection time, thus improving the performance after irradiation by more than an order of magnitude, reducing at the same time the cluster size at the advantage of better two-track separation. Large signal-to-noise ratios are essential for near $100 \%$ efficiency and for implementing features such as time stamping. This can be achieved either with specialized CMOS processes featuring a resistivity in excess of $1 \mathrm{k} \Omega \mathrm{cm}$, or with the SOI process on high resistivity handle wafer. Both approaches have been successfully exploited in monolithic pixel R\&D ensuring signal-to-noise values of 30-40 from minimum ionizing particles at room temperature. The beam telescope developed within the EUDET EU project uses sensors based on high resistivity CMOS pixels thinned to $50 \mu \mathrm{m}$ [10]. They provide a $\sim 3 \mu \mathrm{m}$ single point resolution at a rate of $\sim 10^{4}$ frames/s over an active surface of about $2 \mathrm{~cm}^{2}$ paved with $670 \mathrm{k}$ pixels and can cope with a flux of particles exceeding $10^{6} / \mathrm{cm}^{2} \mathrm{~s}$. The Silicon-OnInsulator process with a high-resistivity handle wafer, developed by an international consortium to which both LBNL and FNAL contribute significantly, offers further appealing opportunities by removing the inherent limitations of bulk CMOS processes. After the pioneering effort in a $3 \mu \mathrm{m}$ CMOS process [11], a commercial $0.2 \mu \mathrm{m}$ SOI process has been made available since a few years by a collaboration of KEK with Lapis Semiconductor Inc. (formerly OKI Semiconductors), Japan [12]. The high resistivity sensitive volume ensures large signals with no interconnections, low collection electrode capacitance and a low-power, potentially radiation tolerant device $[4,13]$. The R\&D has now successfully solved the transistor back-gating problem. Prototype thin SOI pixels of $14 \mu \mathrm{m}$ pitch have been successfully operated overdepleted with a $200 \mathrm{GeV} \pi$ beam at CERN giving a spatial point resolution of $1-2 \mu \mathrm{m}$ and particle detection efficiency above $98 \%$ [14].

Based on a more specialized technology, DEPFET pixels have been developed since the late 80's [15] and integrate a p-MOS transistor in each pixel on the fully depleted bulk. Electrons, collected in the internal gate, modulate the transistor current. Their low input capacitance ensures low noise operation and makes them suitable for a broad range of applications from collider detectors [16] to X-ray astronomy [17]. Thick DEPFET devices of pixel pitch of 20-30um have demonstrated a spatial point resolution of $\sim 1 \mu \mathrm{m}[18]$. 
The design of the electronics for monolithic pixels has to be adapted to the limited amount of collected charge and the need to minimize power. In principle, for monolithic pixel sensors collecting charge from the thin epitaxial layer, the $\mathrm{Q} / \mathrm{C}$ ratio and power consumption might not be necessarily better than that of hybrid pixel detectors. A power consumption and a $Q / C$ improvement can be achieved by minimizing the pixel capacitance $\mathrm{C}$, through a decrease of the size of the collection electrode, and by increasing the charge collection and depletion depth with a moderate- to high-resistivity substrate. Analog and digital power consumption typically differ by less than an order of magnitude in pixel detectors for HEP applications. Analog power reduction by an improved $\mathrm{Q} / \mathrm{C}$ or by power pulsing has therefore to be matched by a similar reduction in digital power. Power pulsing schemes also for the digital readout are considered, in particular for applications at the ILC due to the low duty cycle. Architectures limiting the off-chip data rate and minimizing the number of operations are also under study. As an example, in the LePix project pursued by an European collaboration centered at CERN [19], the in-pixel circuitry is reduced to minimize the pixel capacitance and data from different pixels are combined in different projections and immediately transmitted to the readout electronics on the periphery of the chip. The projections are chosen carefully to minimize ambiguities. Simulations indicate that occupancies of $\sim 50 \sim$ hits $/ \mathrm{cm}^{2}$ can be handled and only $4 \mathrm{~N}$ signals need to be treated at the periphery for an NxN pixel matrix with four projections. An important component of the power consumption is the off-detector data transmission, and any progress to optimize power/data rate in this area will be beneficial to new applications. Time stamping may be key for reducing accelerator-induced backgrounds and $\mathrm{O}(1 \mathrm{~ns})$ resolution has been included in studies for multi$\mathrm{TeV}$ collisions at CLIC and a Muon Collider. More relaxed performances of O(10-100 ns) might be applicable for monolithic pixels at LHC and the ILC. The Chronopixel architecture aims at implementing time stamping of $\sim 300 \mathrm{~ns}$ and data sparsification in the pixel cell of a CMOS monolithic sensor using only NMOS transistors, thus avoiding to depend on special processes [20]. The TimePix architecture, already successfully implemented in hybrid sensors and under study for the LHCb VELO upgrade, may represent an adavantageous starting point for the development of a multi-tier 3D sensor attaining ns resolution.

3D integration technologies promise to provide a very elegant way for implementing advanced readout architectures of a pixel matrix [21]. It offers a solution to the conflict between high pixel granularity and fast readout requirements by integrating heterogeneous technologies with individual tiers, one dedicated to charge collection and others providing signal processing and data reduction with dedicated architectures. Digital electronics can be removed from the silicon layer where the sensing electrodes and the analog front-end circuits are located, eliminating interference due to substrate coupling. In a purely digital layer, the designer is allowed to integrate various advanced readout concepts, such as time-ordered readout of hit pixels, in a "data push" or triggered way, enabling a pixel matrix to deal with a hit rate of $100 \mathrm{MHz} / \mathrm{cm}^{2}$ with a readout efficiency close to $100 \%$ [22]. There are several approaches to 3D integration differing in terms of the minimum allowed pitch of bonding pads between different layers and of vertical TSVs across the silicon substrate. The HEP community focused a large effort on the design of 3D 
integrated circuits with the "via first" technology, where the TSVs are etched in the silicon wafers in the early stages of CMOS fabrication [23], provided by Tezzaron/GlobalFoundries ${ }^{1}$ with significant leadership provided by Fermilab. With the first functional device now at hand, after several production cycles, this is becoming a very promising approach. Even with less aggressive 3D technologies, such as the so-called "via last" processes where TSVs are fabricated on fully processed CMOS wafers, a significant advantage can be gained in most cases, as only one or two connections are needed between the analog and digital blocks of a single pixel cell, and the digital layer can use low-density peripheral TSVs to reach backside bonding pads for external connection. Devices based on the "via last" technique are being studied within the AIDA EU project [24].

Sensors of the thickness routinely achievable with monolithic technologies and back-thinning $(\sim 50 \mu \mathrm{m})$ are compatible with obtaining a material budget of $\sim 0.1 \% \mathrm{X}_{0} /$ layer, needed to meet the value of $\mathrm{b} \sim 10 \mu \mathrm{m} / \mathrm{GeV}$ for the multiple scattering term in the impact parameter resolution, identified as a requirement at a linear collider and also at second-generation b-factories. For sensors of this thickness, the ladder support is of major importance for counteracting the chip warping and insuring the module stability and planarity. In the design of thin ladders for applications at B-factories, STAR and the linear collider with thin sensors, as well as those for an LHC upgrade, the Si ceases to be the largest single factor in the overall layer material budget which becomes the cable routing signals, power and clocks. Therefore, there seems to be limited interest in pushing the sensor thickness below $\sim 50 \mu \mathrm{m}$ in HEP applications. In order to progress towards modules with overall smaller total material budget, sensor stitching, with clocks and signals routed on metal lines in the chip, appears a promising path. The development of very light double-sided ladders, which are advantageous mechanically and offer hit correlation to reject low energy particles from beam background, appears promising. The PLUME collaboration is addressing some of the challenges related to ladders based on thin monolithic pixel sensors.

\section{References}

[1] E. Fossum et al., IEEE Transactions on Electron Devices, 44 (10) (1997), 1689.

R. Turchetta et al., Nucl. Instrum. and Meth. A458 (2001), 677.

[2] G. Traversi et al., Nucl. Instrum. and Meth. A572 (2007), 396.

[3] W. Kucewicz et al., Nucl. Instrum. A542 (2005).

Tezzaron Semiconductor, Naperville, IL, USA;

GlobalFoundries Inc. Milpitas, CA, USA 
[4] Y. Arai et al., Nucl. Instrum. and Meth. A 623 (2010), 186.

[5] N. Neri et al., Nucl. Instrum. and Meth. A623 (2010), 195

[6] S. Zucca et al., Nucl. Instrum. and Meth. A10.1016/j.nima.2012.12.106, in press.

[7] I. Peric, Nucl. Instrum. and Meth. A 650 (2011), 158.

[8] C. Kenney et al., Nucl. Instrum. and Meth. A342 (1994), 59.

[9] M. Battaglia et al., Nucl. Instrum. and Meth. A716 (2013), 29.

[10] J. Aguilar, et al., arXiv:1201.4657.

[11] W. Kucewicz et al., Nucl.Instrum. and Meth. A542 (2005), 172.

[12] Y. Arai et al., Nucl. Instrum. and Meth. A636 (2011), S31.

[13] M. Battaglia et al., Nucl. Instrum. and Meth. A583 (2007), 526.

[14] M. Battaglia et al., Nucl. Instrum. and Meth. A676 (2012), 50.

[15] J. Kemmer, G. Lutz, Nucl. Instrum. and Meth. A253 (1987) 356.

[16] S. Rummel et al., Nucl. Instrum. and Meth. A623 (2010) 189.

[17] A. Stefanescu et al., Nucl. Instrum. and Meth. A624 (2010) 533.

[18] L. Andricek et al., Nucl. Instrum. and Meth. A638 (2011), 24.

[19] S. Mattiazzo et al., Nucl. Instrum. and Meth. A10.1016/j.nima.2012.10.098, in press.

[20] C. Baltay et al., arXiv:1109.2811v1.

[21] R. Yarema et al., Nucl. Instrum. and Meth. A617 (2010), 37.

[22] A. Gabrielli et al., Nucl. Instrum. and Meth. A658 (2011), 141.

[23] R. Yarema et al., Nucl. Instrum. and Meth. A617 (2010), 37.

[24] G. Traversi et al., Journal of Instrumentation, 7 (2012), p. C072007. 


\section{Hybrid Pixel Detector Challenges for next Generation Frontier Experiments at the LHC}

\section{Contribution of Gino Bolla (Purdue) and M. Garcia-Sciveres (LBNL)}

Hybrid pixel technology is used at the LHC in several experiments (ATLAS, ALICE, CMS) and it is the baseline for small radius tracking at planned upgrades. Such technology provides the highest radiation tolerance and rate capability available today. The rate capability is a property mainly of the integrated circuit, as are tolerance to ionizing radiation dose and single event upsets. Tolerance to bulk damage radiation dose is a property of the sensor.

The most advanced hybrid pixel system now being built is the ATLAS IBL upgrade that will be installed in the experiment in 2014. The FE-I4 chip meets the specification of $\sim 300 \mathrm{MHz} / \mathrm{cm}^{2}$ data rates and 300 Mrad of ionizing radiation dose while both planar and 3D sensors has been tested and proven reliable up to the required integrated fluence of $5 \times 10^{15} \mathrm{neq} . / \mathrm{cm}^{2}$. Moreover the ionizing radiation tolerance of FE-I4 (and therefore the 130nm CMOS process used) has been tested to double the required value.

For a High Luminosity LHC the requirements on rate and radiation essentially triple. For the readout chip the driving challenge is meeting the increased rate (order $1 \mathrm{GHz} / \mathrm{cm}^{2}$ ). This implies a need for smaller and faster pixels and at the same time more in-pixel memory and functionality. A readout chip with a higher density process than $130 \mathrm{~nm}$ CMOS is therefore needed. Both 3D chip fabrication and smaller feature size CMOS processes have been investigated. The currently favored solution is $65 \mathrm{~nm}$ CMOS. The main challenges of producing a new chip in this technology are the effort needed to carry out the design and to qualify the new technology to the $1 \mathrm{Grad}$ radiation dose level. We expect $65 \mathrm{~nm}$ CMOS to be rad hard up to such doses, but the qualification work is nevertheless large, because one must, among other things, validate the full digital library in order to enable digital logic synthesis.

A large format pixel IC in $65 \mathrm{~nm}$ will contain $\sim 500$ million transistors, which is more than any single core computer processor ever had (only multi-core processors broke the 1 billion transistor barrier). An IC of this magnitude would need a very large design effort even without considering the extreme radiation tolerance requirement. Such an ambitious goal can only be achieved within our community by pooling resources across experiments and across countries into one focused collaboration. Prototyping in $65 \mathrm{~nm}$ will also be costly and avoiding effort duplication is therefore important. For these reasons a new CERN RD collaboration is being formed. It will focus on the development of a next generation of pixel readout chips in $65 \mathrm{~nm}$. This collaboration will bring together the pixel chip design communities of ATLAS and CMS. This effort will benefit other users of $65 \mathrm{~nm}$ technology as well. In particular design of pixel chips meeting requirements for ILC or CLIC. A separate white paper on IC design is being prepared, covering all HEP activities. 
Purely from a radiation tolerance standpoint, pixel sensor R\&D is very active and both $3 \mathrm{D}$ and planar pixel technologies are being prototyped to meet the $1.5 \times 10^{16} \mathrm{neq} / \mathrm{cm}^{2}$ challenge. The core of this work is done within the RD50 collaboration. Separate white papers are addressing this. This also falls in the category of challenging, but already being addressed.

While rate and radiation are paramount, they are not the only challenges for the next generation of pixel detectors. There are new ones for the next generation of detectors that were not present in the devices that are presently installed along the LHC ring.

- An emerging physics requirement is the need for much better 2-track separation in order to maintain tracking efficiency and $b$-tagging performance for highly boosted jets.

- Reducing mass becomes more important to improve performance in high pileup.

- More efficient power distribution (via on-detector voltage conversion) becomes inescapable to build larger, lower mass detectors.

- Much higher detector output bandwidth will be needed to accommodate higher readout rates (higher rates for the lowest level trigger).

Finally, building larger detectors requires lowering cost and scaling up fabrication capacity. An interesting cost challenge is in fact to realize the potential of flip-chip assembly to be the lowest cost method for fabricating hybrid detectors, as explained later.

Improving 2-track separation requires not only smaller pixels. Already going to higher rate capability required reducing the pixel size, but for separating tracks there is a lower limit to the useful pixel size. This is given by the extent of the charge deposit in the sensor, which is in turn given by the sensor thickness. The $65 \mathrm{~nm}$ pixel chip design is targeting pixel dimensions of order $25 \mathrm{um} \times 150 \mathrm{um}$. For such pixels to be useful the sensor thickness should not exceed $100 \mu \mathrm{m}$. While thin sensors well below $100 \mu \mathrm{m}$ have already been fabricated there is still a need for Sensor $R \& D$ in this direction in order to establish cost-effective means of mass production for such reduced thickness. Moreover more physics understanding is needed to determine whether this development will fulfill the requirements. Continuing to scale hybrid technology to thinner sensors and smaller pixels is problematic. There is a fundamental limitation. The interconnect capacitance in a bump bonded system sets a minimum value for the noise, hence the sensor thickness cannot be reduced further than $\mathrm{S} / \mathrm{N}$ allows. To go thinner the only solution is a monolithic system- either a CMOS sensor or 3D integrated chip plus sensor (no bumps).

$\mathrm{R} \& \mathrm{D}$ into radiation hard CMOS pixels is an actively growing area and could become viable on a timescale needed for the HL-LHC. Two approaches are being pursued: the fully monolithic approach of a CMOS readout chip that is also its own sensor, and the hybrid approach where a rad-hard CMOS sensor replaces the transitional diode based planar of 3D sensor. This latter 
approach takes advantage of all existing infrastructure, but implements a device with an active layer of just 10 to $20 \mu \mathrm{m}$, solving the S/N problem with internal amplification on the CMOS sensor. Interconnection to the readout chip can also be bump-less using capacitive coupling, which simplifies assembly.

Bump bonding interconnection, which may seemingly also need to scale in order to accommodate smaller pixels, is in fact not a challenge. This is because the technology used for present LHC detectors already has an areal density of 40,000 bumps $/ \mathrm{cm}^{2}$ (which correspond to a 50x50 $\mu \mathrm{m}$ squared pixel), which was underutilized. For comparison, the target pixel density of the above mentioned $65 \mathrm{~nm}$ chip is 27,000 bumps $/ \mathrm{cm}^{2}$.

The mass reduction challenge has driven work on mechanics and cooling. $\mathrm{CO}_{2}$ evaporative cooling has been demonstrated and is the baseline for HL-LHC. R\&D into other methods continues in order to see if one can still do better. Recent advances in carbon composites include development of thermally conductive carbon foam.

Power distribution work focuses on the two options of serial connection or DC-DC conversion. For the case of hybrid pixel detectors, DC-DC conversion is only low enough mass if integrated inside the readout chip, or if the readout chip power consumption can be reduced by an order of magnitude. In-chip DC-DC conversion is challenging for conversion ratios greater than 2 or 3 , due to voltage limitations of the CMOS processes used. However a factor of 2 or 3 is not enough for high efficiency power delivery.

Ideally one would like to read out a pixel detector at the full LHC interaction rate of $40 \mathrm{MHz}$, but this is not considered feasible. One could argue that developing a way to read out a detector with $1 \mathrm{GHz} / \mathrm{cm}^{2}$ rate at $40 \mathrm{MHz}$ is therefore a challenge that is not being addressed, and would likely be transformative if solved. Readout at 0.5-1 MHz is currently being considered. This would require output data links of 1 or $2 \mathrm{~Gb} / \mathrm{s}$ per chip for an inner layer at the HL-LHC. This is a practical value for a single serial output in $65 \mathrm{~nm}$ technology. The main challenge at such bandwidth is from the cables, not the chip design. Optical transmission directly from the chip could also be a solution, but note that the trend in new pixel detectors is to increase reliability by moving optical elements as far away as possible from the interaction point.

In the area of cost, the relevant question to pose is: why aren't hybrid pixel detectors cheaper than strip detectors? The whole point of flip chip bump bonding in industry was to reduce assembly cost relative to wire bonding. The mass production cost of readout integrated circuits per unit area is low. A hybrid pixel sensor provides 2 coordinates with a single sensor, while two strip sensors are needed to do the same job. Sensor costs should be half, and flip chip bonding should be cheaper than wire bonding. It could be that as industrial competition increases, the cost of hybrid pixels will in fact naturally come down below that of strips for the same detector area. It is a challenge to understand if this will happen and prepare to take advantage of it. There are currently several manufacturers in Europe and Japan scaling up bump bonding capabilities. A 
major factor in the manufacturing cost is the size of the readout chip and the number of chips per sensor, as the flip chip costs scale with the number of chips, not with physical area. The advent of reticle size chips has already greatly lowered the hybrid pixel cost relative those of the first generation of LHC experiments. 


\section{D Architecture Pixel Sensors}

\section{Contribution of Cinzia Da Via (Manchester) and C. Kenney (SLAC)}

\section{Future Requirements and Challenges}

Planned upgrades to the LHC will result in increasingly harsh radiation environments close to the interaction point. A lepton collider would have similar challenges. Existing semiconductor sensor technologies cannot operate effectively over the duration of an experiment under these radiation conditions. One path to meet this challenge is through the use of 3D or non-planar geometry sensors. Development of such sensors has been on-going since the 1990s and is still an active area of study with a growing impact beyond the Energy Frontier. The selection of 3D-geometry sensors for the ATLAS insertable B-layer of pixels during the 2013-2014 shutdown is an indication of the promise of such technologies. Such sensors can also offer faster charge collection, when for example structured as parallel trenches, than traditional planar sensors. Fast, charge collection maybe also be useful for beam monitors or in time-of-flight systems.

The higher density of tracks resulting from the large multiplicity of interactions per beam crossing will necessitate smaller pixels in order to maintain an acceptable occupancy. To achieve a finer resolution in the $\mathrm{Z}$ direction means thinner sensors to prevent low-angle tracks from hitting many pixels. Thinner sensors will also reduce multiple scattering and photon conversions. Since leakage current is proportional to the thickness of the sensor, power and heat generation will be accordingly less as well. The table below summarizes the attributes that will be needed for a vertex sensor a decade from now.

\begin{tabular}{|l|l|l|l|l|l|l|l|}
\hline & $\begin{array}{l}\text { Radiation } \\
\text { Tolerance }\end{array}$ & $\begin{array}{l}\text { R- } \varphi \\
\text { Spatial } \\
\text { Resolution }\end{array}$ & $\begin{array}{l}\text { Z Spatial } \\
\text { Resolution }\end{array}$ & $\begin{array}{l}\text { Radiation } \\
\text { Length }\end{array}$ & $\begin{array}{l}\text { Time } \\
\text { Resolution }\end{array}$ & $\begin{array}{l}\text { Active } \\
\text { Edges }\end{array}$ & $\begin{array}{l}\text { Integrated } \\
\text { Cooling }\end{array}$ \\
\hline $\begin{array}{l}\text { Vertex } \\
\text { sensors }\end{array}$ & $\begin{array}{l}1 \times 10^{17} \\
\mathrm{n} / \mathrm{cm}^{2}\end{array}$ & $25 \mu \mathrm{m}$ & $25 \mu \mathrm{m}$ & $<0.2 \%$ & $<20 \mathrm{~ns}$ & $\begin{array}{l}<10 \\
\mu \mathrm{m}\end{array}$ & Yes \\
\hline
\end{tabular}

\section{Most promising R\&D paths}

Many attributes of 3D sensors must be improved to optimize the system-level performance of future vertexers. Active-edge or thin-edge designs help to minimize material budget and allow for full hermetic coverage. Devices with insensitive borders under 10 microns have been fabricated and confirmed in test beams. This can have a dramatic effect on tracking efficiency. Incorporation of such techniques into production devices is critical. 
A significant improvement in radiation tolerance may be achievable by incorporating limited internal gain into the sensor via avalanche multiplication. This would be accomplished by performing extensive calculations to explore the device geometry, biasing, and diffusion parameter space. Prototype runs to confirm the simulation results with follow-on irradiations and beam tests to qualify the concept and final sensors would be needed.

Radiation tolerance could also be improved by reducing the distance between $\mathrm{n}$ and $\mathrm{p}$ type electrodes. This involves using the most advanced plasma-etching machines and carefully developing the deep-etch recipes to maximize the achievable aspect ratio. Studying techniques to make the electrodes sensitive to ionizing tracks would help both the track efficiency and allow closer electrode spacing. It should be noted that the 3D architecture has been extended to diamond sensors and that this is an active area of research.
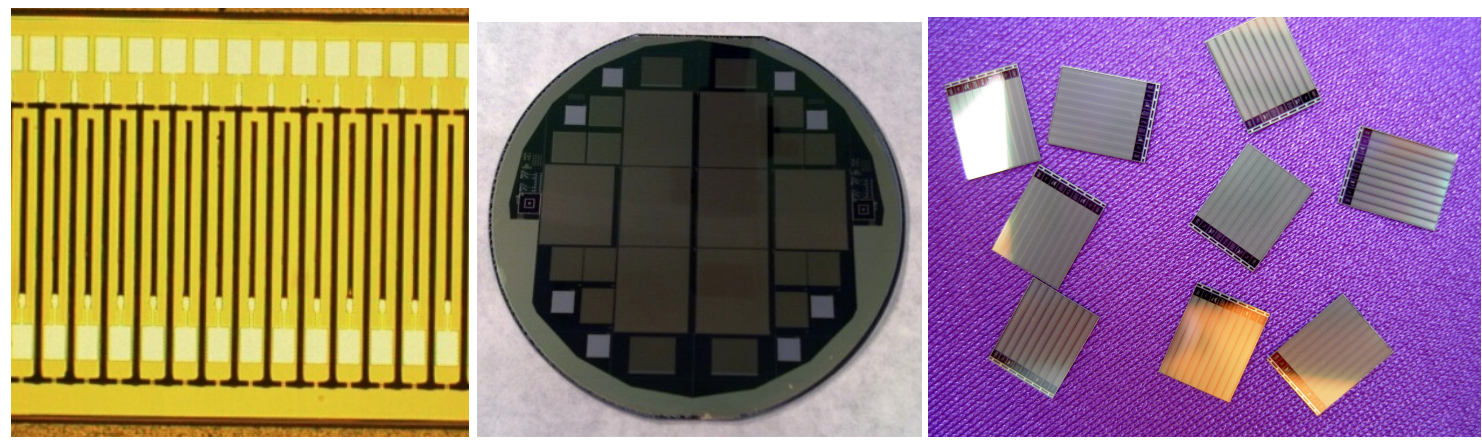

Figure 1. Interleaved, fine-spaced trenches for fast response made at Stanford (left), production wafer with ATLAS IBL sensors made at CNM (center), ATLAS FE-I3 3D sensors with active edges (right).

An important feature of 3D sensors are their moderate power dissipation after irradiation, due to the reduced bias voltage needed to get the electric field across the full substrate thickness. This can prove crucial in the design of a low mass system where services, including cooling, might introduce a considerable amount of mass. Micromachining, the same technology used for etching the 3D sensors electrodes, can be used to etch micro-channels, or micro-grooves, in the post process thinned electronics chip substrate. Images of prototype cooling channels integrated into silicon chips are shown in Figure. 2.
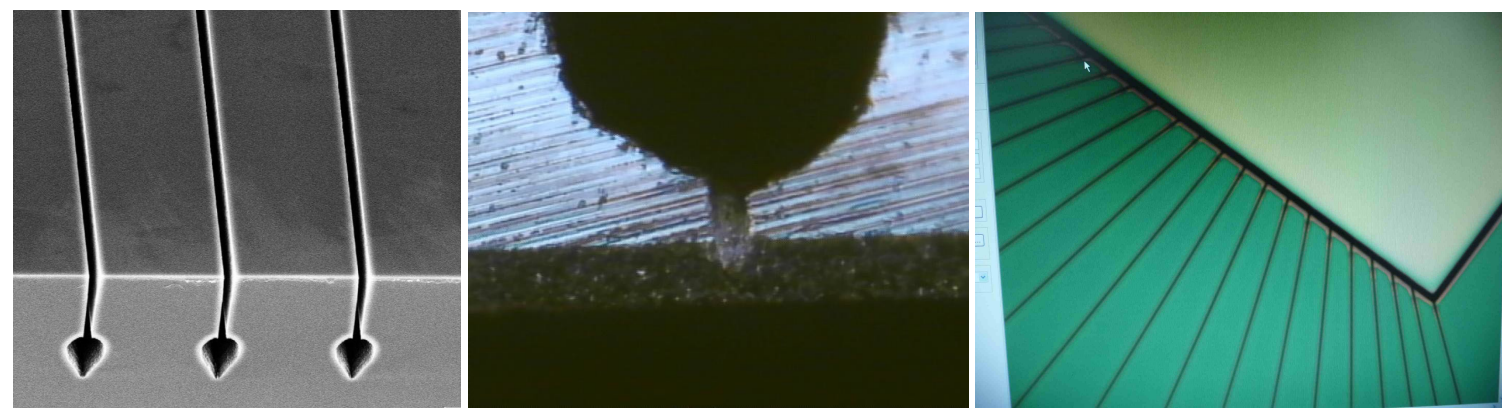
Figure 2. Micro-channels etched at FBK for the SUPERB project in collaboration with F. Bosi and co-workers in silicon. The micro-channel is $\sim 50$ microns in its larger section. (left) close up showing a glass film sealing the micro-channel aperture. (center) micro-cooling channels emptying into a input manifold made at Stanford (right).

\section{Scalability and Costs}

In preparation for construction of the ATLAS insertable B-layer, a consortium of foundries (e.g. CNM, FBK, SINTEF, and Stanford) was organized and has proved very successful in scaling up production of 3D sensors. As the community gains more experience the yields have gone up dramatically with a concordant decrease in cost per unit of sensor area. The micro-machining techniques employed in their fabrication have become ubiquitous in industry and so the opportunity to further partner with commercial vendors is growing. 


\section{Diamond Sensors}

\section{Contribution of Harris Kagan (OSU), William Trischuk (Toronto)}

Luminosity monitors, beam monitors and tracking detectors in future collider experiments, including LHC upgrades must operate in radiation environments several orders of magnitude harsher than those of any current detector. As the environment becomes harsher detectors not segmented, either spatially or in time, become challenged to separate signal from background. Present tracking detectors close to the interaction region are based on highly segmented silicon sensor technology. Chemical Vapour Deposition (CVD) diamond has a number of properties that make it an attractive alternative for high-energy physics detector applications. Its large band-gap $(5.5 \mathrm{eV})$ and large displacement energy ( $42 \mathrm{eV} /$ atom) make it a material that is inherently radiation tolerant with very low leakage currents and can be operated near room temperature simplifying the mechanical support and cooling necessary. The drawback of using diamond is that it only provides $60 \%$ of the number of charge carriers, per unit radiation length of thickness, relative to silicon. However the steady luminosity increase at colliders, in particular at the LHC, justify considering diamond as a replacement for $\mathrm{Si}$ in the innermost layer(s) of the vertex trackers. CVD diamond is being investigated by a number of groups for use very close to LHC interaction regions, where the most extreme radiation conditions are found. Here we describe the requirements for inner tracking sensors at future colliders, highlight the $R \& D$ on diamond sensors underway and conclude with a discussion of scalability and cost.

In order to continue the physics studies at the LHC it is imperative that precision tracking continue to be present at the smallest possible radii. In practice, this means the sensors must continue to operate efficiently after fluences of $10^{16}$ charged particles per $\mathrm{cm}^{2}$. At these inner radii minimizing the amount of material in the tracker is paramount as excess scattering and pair conversion significantly compromise pattern recognition and further exacerbates occupancies. Diamond sensors do not require extensive cooling and, being low $\mathrm{Z}$, present less material per unit thickness than silicon or other solid-state tracking media. Of course, to be viable it must be possible to pixelate the diamond sensors, read them out with integrated VLSI electronics and produce reasonably large arrays affordably.

Table 1 shows a list of sensor requirements that would make diamond a viable alternative for future energy frontier trackers. In what follows we describe the R\&D aimed at demonstrating that diamond is a viable sensor material for these applications. Although diamond sensors have recently been targeted at collider detector applications, their first use in HEP was in B-factory background monitoring. They continue to be good candidates for beam diagnostics at intensity frontier machines. 
Table 1: Diamond sensor specifications

\begin{tabular}{|l|l|}
\hline Requirement & Specification \\
\hline Sensor size & $5 \times 5 \mathrm{~cm}^{2}$ \\
\hline Sensor Thickness & 400 microns \\
\hline Minimum charge mean free path & 250 microns \\
\hline Minimum average charge before irradiation & 9000 electrons \\
\hline $\begin{array}{l}\text { Minimum mean free path/ } \\
\text { charge after } 10^{16} \mathrm{~cm}^{-2}\end{array}$ & 150 microns/ \\
\hline Minimum signal/threshold after $10^{16} \mathrm{~cm}^{-2}$ & 5400 electrons \\
\hline Single hit efficiency & 3 \\
\hline Spatial resolution & $>99.9 \%$ \\
\hline Maximum operating voltage & $<14 \mu \mathrm{m}$ \\
\hline Maximum total leakage current (@1000 V) & $1000 \mathrm{~V}$ \\
\hline
\end{tabular}

Significant progress in diamond sensor R\&D has been made over the last five years as the LHC experiments have come on line. All four LHC experiments include diamond beam monitors to protect them from un-stable beam conditions. The most sophisticated of these, in the ATLAS experiment, are also used as the default luminosity counters providing collision and background counting rates that have been stable to better than $1 \%$ over the first three years of LHC operation. Reading out the diamond with sub-ns shaping-time electronics has been crucial to achieving this stability in ATLAS as it allows a clear distinction between signals in-time with bunch crossings and background particles that arrive out-of-time. The RD42 collaboration has performed extensive irradiations of CVD diamond material with protons ranging from $26 \mathrm{MeV}$ to $24 \mathrm{GeV}$, pions at $300 \mathrm{MeV}$ and reactor neutrons. Figure 1 shows the change of carrier mean free path (signal produced when MIPs traverse the sensor) in state-of-the-art CVD diamond material (typical mean free paths of 300 microns in PCVD material) showing superior radiation tolerance to fluences beyond $10^{16}$. 


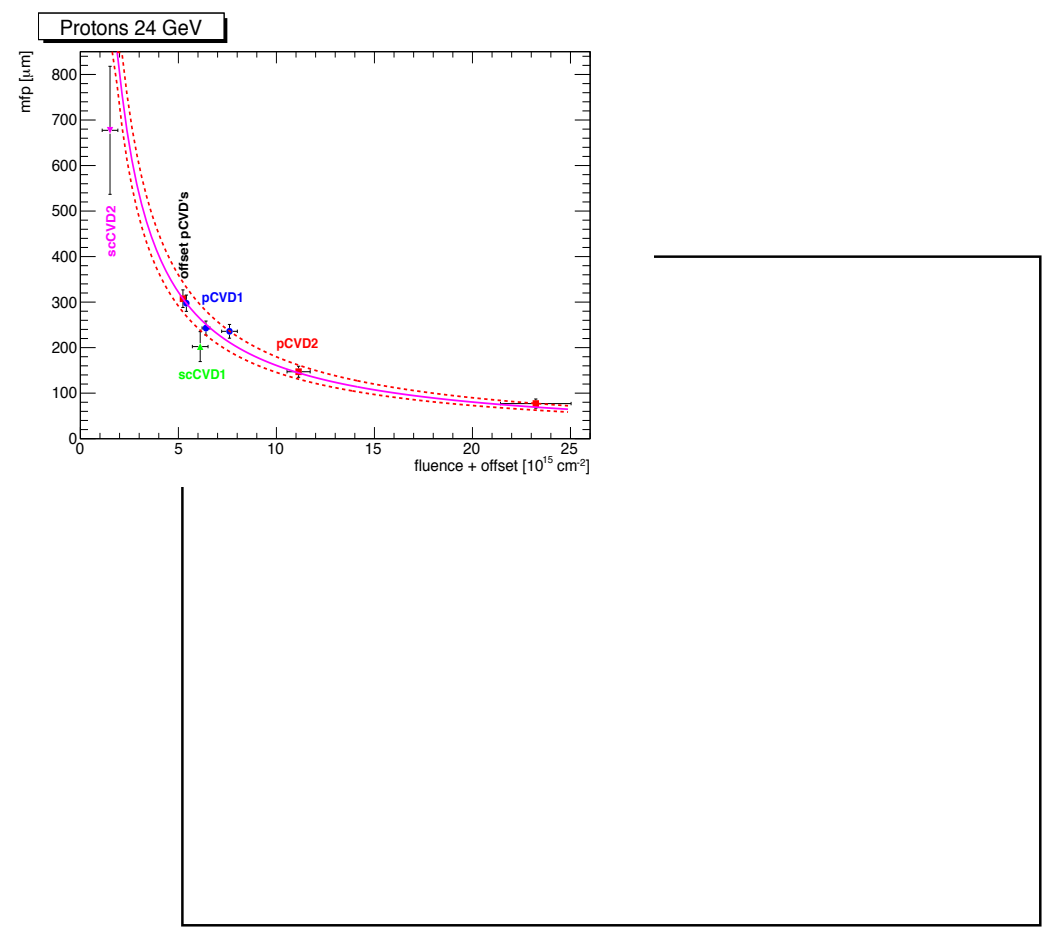

Figure 1: Mean free path of charge carriers in CVD diamond sensors vs. fluence of $24 \mathrm{GeV}$ protons. All types follow a common degradation due to the appearance of additional traps. pCVD material is offset to reflect the level of traps in its as-grown state.

Over the last five years there has also been good progress on scaling up the growth of CVD diamond material. Several manufacturers have demonstrated the capability of growing wafers $10 \mathrm{~cm}$ in diameter (or larger) with charge collection distances that are suitable for single MIP tracker applications. As a result prices have started to come down although they still remain higher than for silicon sensors - although silicon sensors that might survive the dose necessary to survive in future LHC pixel detectors are more expensive due to the elaborate processing they require. In addition to learning how to grow the materials manufacturers have learned that surface preparation is important to the overall quality of the sensors and the long-term survival of electrical contacts to the resulting sensors. The CMS experiment is preparing a set of forward pixel telescopes, based on the largest single crystal CVD diamond sensors currently available 0.5 x $0.5 \mathrm{~cm}^{2}$ ) for installation during the current LHC machine stop. In turn ATLAS is preparing a pixel Diamond Beam Monitor using larger poly-crystalline CVD diamond sensors $\left(1.8 \times 2.1 \mathrm{~cm}^{2}\right)$ on a similar timescale. Both of these projects have demonstrated routine large-scale bumpbonding of VLSI pixel readout chips to diamond sensors and aim to demonstrate routine tracking in the most demanding environment yet explored, less than $5 \mathrm{~cm}$ from the LHC beamline and $1 \mathrm{~m}$ from their, respective, LHC interaction points. A number of studies have been performed to characterise the tracking performance with diamond sensors. Charge sharing has been demonstrated with the diamond strip trackers giving position resolutions of better than $9 \mu \mathrm{m}$ [1] and similar resolution has been seen in 50um wide pixels in small single crystal pixel devices [2]. Resolution has been shown to improve in polycrystalline CVD sensors after irradiation as the introduction of defects improves the material uniformity [3]. 
The development of diamond sensors for HEP applications has piggy backed on industrial production capabilities for other applications. CVD substrates have applications in thermal management, X-ray windows and, potentially, dosimetry (with Carbon being a better match for human tissue than other solid-state sensor materials). Over the past ten years the RD42 collaboration has worked with a dozen different manufacturers. At the present time we have two viable suppliers (Element6 and II-VI Ltd.). Both have the capability to produce tracker quality substrates and have demonstrated the capacity to produce several hundred $\mathrm{cm}^{2}$ per year of sensor material. Figure 3 shows the size of the various diamond systems that have been installed over the last 10 years. The current capacity, and the potential manufacturers have to increase that capacity, should be sufficient to build two or three tracking layers at radii up to $10 \mathrm{~cm}$ in both the multi-purpose LHC experiments prior to the ultimate luminosity running in the next decade.

Diamond sensors have proven highly reliable replacements for silicon in every HEP application they've been used in over the past 10 years. In each case they've allowed measurements that were unexpected when they were first proposed. We expect that they will continue to revolutionize the way we do tracking in high occupancy, high radiation environments at the high luminosity LHC and beyond.

\section{References}

[1] W. Adam et al., Nucl. Inst. Meth. A565 (2006) p278.

[2] M. Mathes et al., JInst 3 (2008) P12002.

[3] W. Adam et al., Nucl. Inst. Meth. A514 (2003) p79 


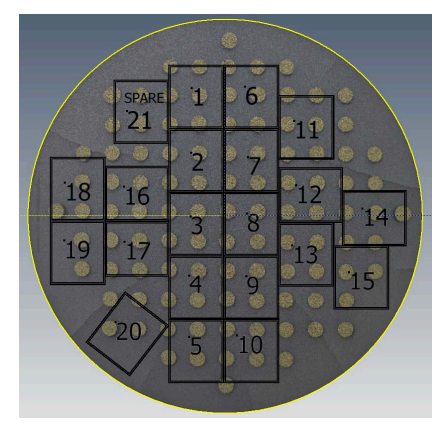

Figure 3: Active area of various diamond detector systems that have come in to operation over the last ten years. The open symbols show tracking devices that are currently under construction.

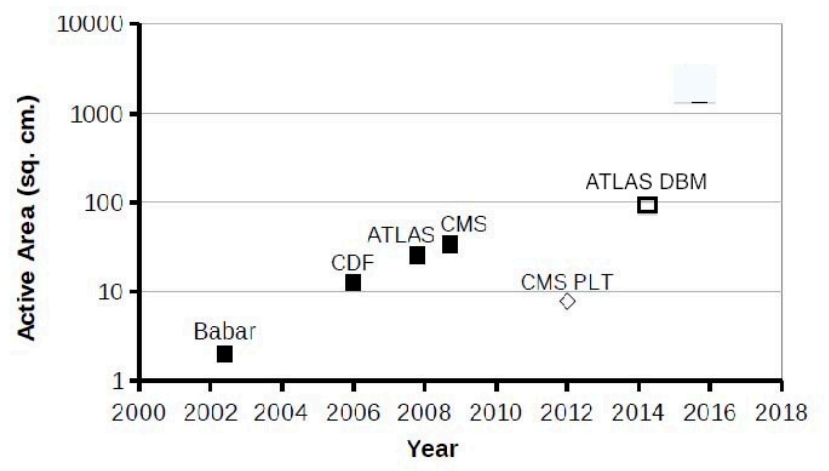




\section{Ultra-fast Silicon Detectors (4D-UFSD) \\ Contribution of Hartmut Sadrozinski (UC Santa Cruz)}

\section{Principle of 4D-UFSD}

The ultimate goal of 4D-UFSD is to establish a new paradigm for space-time particle tracking by developing a single device that matches the performances of the most accurate position and time detectors. The goal is to obtain a position resolution of $\sim \mathbf{1 0} \boldsymbol{\mu} \mathbf{m}$ while measuring the arrival time of the particle with an uncertainty of $\sim \mathbf{1 0}$ ps. In the past, precise tracking devices have determined time quite poorly while good timing devices are too large for accurate position measurement.

The goal is to develop the $4 \mathrm{D}$ capability by augmenting the superb position resolution of a segmented silicon sensor with high-resolution timing information.

The 4D-UFSD principle is based on

a) Use of thinned silicon pixel/strip sensors to reduce the charge collection time.

b) Increase the charge presented to the read-out electronics by employing charge multiplication within the sensor bulk.

c) Develop a read-out system that will match the silicon sensor rate capability, segmentation and time resolution.

\section{Gain in Silicon Sensors}

Given that the drift velocity in silicon saturates at about $10^{7} \mathrm{~cm} / \mathrm{sec}$, the collection time of electrons inside a silicon layer of $\sim 300 \mu \mathrm{m}$ is $\sim 3 \mathrm{~ns}$. Much faster silicon sensors need therefore to be very thin which means that the charge collected from a thin active layer might not be sufficient to achieve good time resolution. We propose to exploit charge multiplication to increase the charge yield of very thin silicon sensors so that they can generate ultra-fast timing signals [1]. The past observation by several RD50 groups [2] of moderate gain in irradiated silicon sensors, and the new measurements on un-irradiated pad detectors with optimized implants showing gain of about 10 [3][4] creates the opportunity for producing thin, very fast silicon sensors, which can work at extremely high-rates without dead-time issues.

It has been demonstrated that in silicon sensors the charge multiplication factor $\alpha$, which is responsible for the charge gain, has an exponential dependence on the inverse of the electric field [5]. At the breakdown field, $E_{\max }=270 \mathrm{kV} / \mathrm{cm}, \alpha \sim 0.7 / \mu \mathrm{m}$ for electrons and $\alpha \sim 0.1 / \mu \mathrm{m}$ for holes, which limits the maximum achievable gain g. $N_{0}$ electrons, drifting a distance $d$, with a charge multiplication factor $\alpha$ become $N_{\text {Tot }}$ electrons: 


$$
N_{\text {Tot }}=N_{0} e^{\alpha * d}=g N_{0}
$$

For example, electrons drifting $5 \mu \mathrm{m}$ in the maximum field generate a gain $\mathrm{g}=\mathrm{N}_{\text {Tot }} / \mathrm{N}_{0}=33$. Changing the field by $5 \%$ changes the gain by more than a factor 2 .

\section{Sensor Thickness: Capacitances, Collected Charge, Collection Time, Gain}

The thickness of the active area determines several key parameters of the sensor. Considering two possible geometries, (i) a $50 \mu \mathrm{m}$ x $50 \mu \mathrm{m}$ pixel and (ii) a one mm-long strip with $50 \mu \mathrm{m}$ pitch, Table 1 shows the backplane capacitance, the number of electrons that form the signal based on [6], the collection time, and the gain required to reach an acceptable signal level, 2000 electrons for the pixel, and 12000 electrons for the strip sensor [7].

Taking values of the inter-strip capacitance for existing pixel sensors (200 fF) and n-on-p strip sensors ( $1 \mathrm{pF} / \mathrm{cm})$ as a guideline, a 4D-UFSD thickness where the backplane capacitance would not dominate are $2 \mu \mathrm{m}$ for pixels and $5 \mu \mathrm{m}$ for strips.

From the values shown in Table 1, pixel sensors offer very attractive combinations of moderate gain, small capacitance, and short collection time. Due to the high value of backplane capacitance, strip sensors cannot be made as fast as pixel sensors; however, a "mini-strip" of 1-2 mm long offers a quite fast collection time, $50-100 \mathrm{ps}$, with a moderate value of capacitance ( $1 \mathrm{pF})$.

\begin{tabular}{|c|c|c|c|c|c|c|}
\hline $\begin{array}{c}\text { Thickness } \\
\text { [um] }\end{array}$ & \multicolumn{2}{|c|}{ BackPlane Capacitance } & Signal & Coll. Time & \multicolumn{2}{c|}{ Gain required } \\
\hline Pixels [fF] & Strips [pF/mm] & [\# of e-] & [ps] & for 2000 e & for 12000 e \\
\hline 2 & 125 & 2.5 & 80 & 25 & 25 & 149 \\
5 & 50 & 1.0 & 235 & 63 & 8.5 & 51 \\
10 & 25 & 0.50 & 523 & 125 & 3.8 & 23 \\
20 & 13 & 0.25 & 1149 & 250 & 1.7 & 10.4 \\
100 & 3 & 0.05 & 6954 & 1250 & 0.29 & 1.7 \\
300 & 1 & 0.02 & 23334 & 3750 & 0.09 & 0.5 \\
\hline
\end{tabular}

\section{Sensor Processing Options}

An important research activity is the optimization of the sensors for stable processing and operations. This includes efforts to both better quantify what is physically happening in the sensor and to tailor the field to optimize the performance for timing purposes and for minimum material. The gain phenomenon in segmented silicon sensors is in need of deeper understanding, and then to translate this understanding into processing.

\section{a. Wafer type p-on-n vs. n-on-p}

Several arguments favor n-on-p sensors (which collect electrons) over p-on-n (which collect holes): the collection of electrons is faster, electrons have a factor of 10 larger multiplication gain [5] and there have been many studies on radiation-hardness of p-type sensors for the LHC upgrade [7][8][9]. 


\section{b. Epitaxial and Thinned Float Zone}

Thin epitaxial sensors are easily produced since they consist of a low-resistivity electrode $\left(\mathrm{n}^{++}\right)$ implanted in a high-resistivity $\mathrm{p}^{-}$epitaxial layer of silicon, deposited on a thick low-resistivity $\mathrm{p}^{++}$ substrate. Given the low costs and quick manufacturing turn-around time, thin epitaxial sensors are an ideal low-cost vehicle for prototyping, e.g. for optimizing the field configuration and other key parameters. Ultimately one wants to eliminate the thick "handle wafer" and migrate to thinned FZ sensors. This process uses a FZ wafer of the usual thickness of several hundreds of micro-meters and removes the excessive material at the backside by etching. Several groups have produced back-etched thin sensor, down to $15 \mu \mathrm{m}$ thickness, using a selective etch to create ribs for mechanical strength [10][11].

\section{c. Planar and 3D}

Planar sensors collect charges in implants on the surface, while 3D sensors [12] collect charges in columns implanted in the sensor bulk. For 3D sensors, there are good theoretical arguments [13] and supporting their use as fast sensors with gain. The main problem might be that it would be difficult to achieve the tuning of the field around the narrow columns with the precision described in the next section.

\section{d. Tuning of the Electrical Field}

The field profile in the sensors is determined by the doping profile and the bias voltage. Fine tuning of the doping profile is required to simultaneously satisfy the different requirements.

\section{i. Minimum Field}

One of the requirements is that in order for the electrons to be moving at the highest possible velocity, the field needs to exceed $25 \mathrm{kV} / \mathrm{cm}$, the field required for saturating the drift velocity [14].

\section{ii. Depth Doping Profile}

In February 2013, RD50 groups [3][4] have reported gain in un-irradiated pad sensors using specialized deep n++ implants with underlying p+ diffusion [15] (Fig. 1). Adopting the same principle to thin sensors, one can establish a high-field region close to the collecting $n++$ implant providing the charge multiplication, with a somewhat lower field region in the back of the sensors providing the charge to be amplified. Figure 2 shows the principle of such a field configuration. An important property of this figuration is that the "amplification field" strength is essentially independent of the bias voltage, thus providing for stable operation and low currents. Charges generated in the bulk and drifting to the $n++$ implant have to pass through this high filed area and will be amplified. Tuning the doping density within this amplification field will be a major R\&D task. 

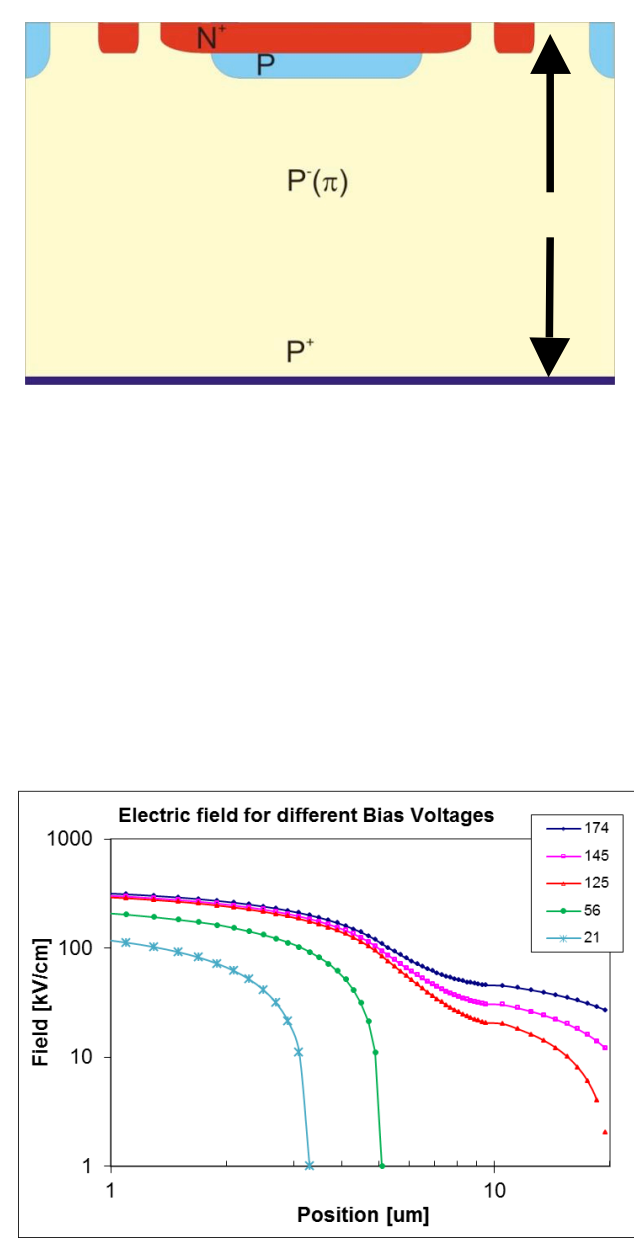

\section{iii. Lateral Doping Profile}

Moving from pads to segmented sensors and pixels raises the problem of lateral field uniformity across the $n++$ implants. Without careful design of the doping profile, charge multiplication happens only in small parts of the sensor, for example near the implant edges where the field lines become very dense. Thus only the charges arriving at the implant edges would be amplified at moderate bias voltages, and when the bias is raised to achieve charge multiplication at the center of the implants, the strip edges would experience electrical breakdown. It is therefore necessary to change the geometry of the field lines inside a sensor in order to prevent this problem, while achieving a large enough volume with high field. By tailoring the depth and/or doping concentration of the additional $\mathrm{p}^{+}$diffusion layer, it is possible to adjust the maximum 
electrical field across the strip width, which can lead to approximately equal multiplication mechanism across the electrode.

\section{References}

[1] H. F.-W. Sadrozinski, "Exploring charge multiplication for fast timing with silicon sensors", $20^{\text {th }}$ RD50 Workshop, Bari, Italy, May 30 - June 1, 2012, and references therein. https://indico.cern.ch/conferenceOtherViews.py?view=standard\&confId=175330

[2] RD50 collaboration, http://rd50.web.cern.ch/rd50/and references in [1].

[3] M. Baselga Bacardit, et al, "Fabrication of new p-type pixel detectors with enhanced multiplication effect in the n-type electrodes", 8 ${ }^{\text {th }}$ Trento Workshop, Feb 18-20, 2013.

http://indico.cern.ch/contributionDisplay.py?contribId=13\&sessionId=4\&confId=226647

[4] G. Kramberger, private communication.

[5] W. Maes, K. De Meyer and R. Van Overstraeten, "Impact ionization in Silicon, a Review and Update”, Solid-State Electronics Vol. 33, No. 6, pp. 705-718, 1990.

[6] S. Meroli, D. Passeri and L. Servoli, "Energy loss measurement for charged particles in very thin silicon layers”, 11 JINST 6 P06013.

[7] A. Affolder, et al., "Silicon detectors for the sLHC", Nucl.Instrum.Meth.A658:11-16, 2011.

[8] M. Bruzzi, H. F.W. Sadrozinski, A. Seiden, "Comparing radiation tolerant materials and devices for ultra rad-hard tracking detectors”, Nucl.Instrum.Meth.A579:754-761, 2007.

[9] Y. Unno, et al., "Development of n-on-p silicon sensors for very high radiation environments", Nucl.Instrum.Meth.A636:24-30, 2011.

[10] M. Lozano, "IMB-CNM Radiation detectors activities", AIDA Project kick-off meeting, CERN, Feb 2011. http://indico.cern.ch/conferenceDisplay.py?confld=125221

[11] M. Beimforde, et al., "First characterizations of thin SOI and epitaxial n-in-p sensors, 14th RD50 Workshop, Freiburg, Germany, 2009. http://indico.cern.ch/conference0therViews.py?view=cdsagenda\&confId=52883

[12] S. I. Parker, C. J. Kenney, and J. Segal, “3D-A proposed new architecture for solid state radiation detectors", Nucl. Instr. Meth, A395:328-343, 1997.

[13] S. Parker, et al., "Increased speed: 3D Silicon Sensors; Fast Current Amplifiers", IEEE Trans. Nucl. Sci., no 58 (2011) 404. 
[14] G. Kramberger, et al., "Electric field and space charge in neutron irradiated n+-p sensors", 19th RD50 Workshop CERN, Switzerland, Nov 21- 23, 2011.

http://indico.cern.ch/getFile.py/access?contribId=7\&sessionId=1\&resId=0\&materialId=slid es\&confId $=148833$

[15] P. Fernandez, et al, "Simulation of new p-type strip detectors with trench to enhance the charge multiplication effect in the n-type electrodes", Nucl. Instr. Meth, A658:98-102, 2011. 


\section{Next Generation Strip Detectors for the Energy Frontier Contribution of Daniela Bortoletto (Purdue University)}

The introduction of Silicon Microstrip detectors in particle physics has been critical for many major discoveries from the top quark to the Higgs. Future experiments operating at high-energy colliders will continue to rely on this technology since the robust tracking and detailed vertex reconstruction that can be achieved with solid state detectors are of central importance to the physics that can be accessed at future hadron colliders including the HL-LHC and the HE-LHC. Solid state detectors can maintain low occupancy, and provide precise and efficient determination of charged-particle momenta which impacts the measurement of all physics objects from leptons, to charged hadrons, jets, and photon conversions. In order to be sensitive to physics involving the third generation and higher mass objects we must achieve efficient $b$ tagging for high momentum jets.

\section{Low mass mechanical supports and cooling systems}

The material in the current tracking systems pose one of most severe limitation on the performance of the detector. It is often dominated by electronics and service components and for example in CMS it presents a major problem especially in the transition region between barrel and end-cap. Furthermore at the HL-LHC the number of particles produced is expected to increase substantially, causing higher occupancy, and increased radiation damage. As a result the power consumption of new trackers is expected to be even larger. $\mathrm{CO}_{2}$ cooling systems are expected to enable a reduction of material inside the tracking by providing an effective and low mass alternative to the current cooling infrastructure. R\&D for the development of new integrated approaches, including powering, and cooling is therefore critical for the HL-LHC.

ATLAS has developed a strawman design of the strip system for the HL-LHC [1]. The stave (petal) is the basic mechanical element of the barrel (end-cap). The barrel module consists of a low mass central core that provides mechanical rigidity and houses the common electrical, optical and cooling services. In order to reduce the material, modules are constructed by directly gluing kapton flex hybrids to silicon sensors with electronics-grade epoxy. The design uses the large cross-sectional area of the hybrid and sensor to the stave core for cooling. The cooling pipes are embedded in a carbon fiber honeycomb sandwiched between two carbon fiber facings. The carbon fiber has both high tensile modulus and high thermal conductivity. The \% radiation length is 1.98 for the barrel (1.60 for the end-caps) in comparison with $2.48 \%$ for the barrels (3.28\% for the end-caps) for the HL-LHC and the current SCT respectively.

ATLAS is conducting R\&D to develop a reliable and low-mass connection method for the several thousand $\mathrm{CO}_{2}$ connections needed [1]. Several options are being investigated, including direct orbital welding of thin-wall Ti tubes; brazing stainless end-pieces to the $\mathrm{Ti}$ and welding to 
standard stainless welding connectors; and developing low mass in-house connectors that can be disconnected and re-used. For in-situ welding, tests are under way to ensure the front-end electronics are not damaged by the process.

CMS is also conducting $R \& D$ on the mechanical modules [2,3]. The concepts under development enable the correlations between top and bottom sensors necessary to provide trigger information. They include strip-strip ( $\mathrm{p}_{\mathrm{T}}-2 \mathrm{~S}$ ) and pixel-strip ( $\left.\mathrm{p}_{\mathrm{T}}-\mathrm{PS}\right)$ modules. The current implementation of the $\mathrm{p}_{\mathrm{T}}$-PS modules has an average radiation length of $2 \%$. A more elegant but challenging approach to the construction of a pT module is represented by the Vertically integrated Pixel-Strip module (or $\mathrm{p}_{\mathrm{T}}$-VPS). In this design one 3D chip reads out the pixelated sensor and the short-strip sensor, connected by analogue paths through an interposer, and implements the correlation logic. The use of vertical connectivity removes all constraints between module dimensions and sensor segmentation. On the other hand, the interconnection technologies are challenging: feasibility, reliability and yield for large-surface assemblies need to be verified, especially considering the demanding operating conditions inside CMS. In addition, in this concept an interposer covers the entire surface of the module, defining the spacing between the two sensors, providing the top-to-bottom connectivity and carrying at the same time power lines and readout signals. The realization of a lightweight interposer with the needed thickness (about $1 \mathrm{~mm}$ ) and electrical and mechanical properties is a key issue to realize a module with an acceptable material density.

\section{Electronic Readout}

The R\&D for the readout of the HL-LHC strip tracker is currently focused on the $130 \mathrm{~nm}$ CMOS process. The CMS CBC2 ASIC [4] is designed to instrument double layer modules in the outer tracker, consisting of two overlaid silicon sensors with aligned microstrips, and incorporates the logic to identify L1 trigger primitives in the form of "stubs" as described in section 5. The ROC will adopt bump-bonded C4 pads, so that routing and pitch adaptation of the input signals from the sensors can be made in an advanced hybrid technology. The logic analyses on adjacent strips from the same sensor allow rejecting wide clusters of hits associated with low-momentum tracks. The coincidence between inner sensors and outer sensors can be adjusted according to the radii and the position of the strips along the module. Care has been taken to keep the power consumption low. The power consumption of the stub finding logic depends on occupancy, maximum cluster width and coincidence window width; however simulations in pessimistic conditions ( $4 \%$ strip occupancy, maximum cluster and coincidence widths) indicate a value of less than $50 \mu \mathrm{W} /$ channel. A total power of less than $300 \mu \mathrm{W} /$ channel for $5 \mathrm{pF}$ load has been achieved. ATLAS has prototyped the ABC130 (130nm CMOS ASICs) [5] which is compatible with both serial powering and DC-DC convertors.

A challenge of the electronic chain is the transmission of large volume of data. Both ATLAS and CMS are currently planning to use the GigaBit Transceiver (GBT) radiation-hard bi-directional 4.8 $\mathrm{Gb} / \mathrm{s}$ optical fiber link [6] to transmit the data between the counting room and the experiments. 
Currently R\&D is needed to provide a version of the low power version of the GBT which will be designed using $65 \mathrm{~nm}$ technology and will only use $1 / 4$ of the power of the current GBT, i.e. but otherwise will use the same protocol and achieve the same speed.

\section{Power Distribution}

Future Collider trackers require large granularity and therefore the power delivery problem is especially challenging. Both serial powering and DC-DC powering are being considered by ATLAS while CMS is focusing only on the latter. Serial powering uses shunt regulators on each module to generate the voltages needed by the front-end ASICs. A Serial Power Protection ASIC (SPP) is being designed [7] that in combination with pass transistors built into the ABC130 will act as a shunt regulator. Prototype staves have been built also with commercial shunt regulators.

DC-DC conversion is a realistic option for improving power delivery efficiency. A buck converter is a circuit topology that can transform DC power using only one inductor. The energy is pumped into the magnetic field at the higher voltage and withdrawn at the lower voltage. If the buck converter operates at a high frequency, the required inductance can be quite small, and an air core inductor can be used in high magnetic fields. CMS is already producing buck converter prototypes, named AC PIX V8, based on the most recent ASIC prototype, AMIS4 [8]. A toroidal plastic-core inductor with an inductance of $450 \mathrm{nH}$ is used, and the switching frequency is configured to $1.5 \mathrm{MHz}$. The fully populated converter boards have a weight of about $2 \mathrm{~g}$. The DCDC converters are equipped with a shield.

DC-DC regulators are used for a large variety of commercial applications and therefore large R\&D investments have been made by the semiconductor industry over the last 50 years. The industry continually strives to produce more efficient and higher frequency power semiconductor devices. Furthermore portable devices such as smart phones must produce several different voltages and therefore they require very small, lightweight DC-DC converters. Currently several companies are developing Gallium Nitride on Silicon which will enable DC to DC converters operating at higher frequencies than Silicon devices. GaN devices are expected to be rad hard and will be used in space and satellite hardware and in military electronics. It is important for future experiments to look if some of the industry advances can be used effectively in future trackers [9].

\section{Sensor and Radiation Hardness}

One of the key issues for strip sensors deployed in a future tracker is to withstand fluences of the order of $2 \times 10^{15} \mathrm{n}_{\mathrm{eq}} \mathrm{cm}^{-2}$. While these specifications are not as stringent as for pixel detectors, R\&D is still necessary in order to find cost effective radiation hard sensors. Dedicated R\&D has been conducted both by ATLAS and CMS. The CERN-RD50 collaboration has also been instrumental in the study of the radiation issues for the HL-LHC.

CMS is conducting a complete measurement campaign to qualify sensor technologies suitable for the HL-LHC [10]. Sensors on 6-inch wafers were produced by Hamamatsu Photonics with different substrates, thickness, implants and geometries. Three different technologies for the 
substrate production are investigated, with different active thicknesses: Float-zone $(120,200$, $300 \mu \mathrm{m})$, Magnetic Czochralski $(120 \mu \mathrm{m})$ and epitaxial $(50,100 \mu \mathrm{m})$. For each substrate technology wafers were produced with three doping types (n-type bulk, p-type bulk with p-spray or p-stop isolation). Multi-geometry strip detectors are the largest structures with $3 \mathrm{~cm}$-long strips. Arrays of long pixels (approximately $1 \mathrm{~mm}$ long) that can be read out by a bump-bonded chip and used to directly measure the track z coordinate are also been implemented.

ATLAS has focused the R\&D on strip sensors that are AC-coupled with n-type implants in a p-type float-zone silicon bulk (n-in-p FZ). These sensors collect electrons and they do not undergo radiation induced type inversion. The standard sensors are about $320 \pm 15 \mu \mathrm{m}$ thick, but the sensor thickness could be reduced to $250 \mu \mathrm{m}$ for a higher cost [1].

The initial measurements that have been conducted indicate that the n-on-p sensors match the requirements of the HL-LHC. There has also been progress in the understanding of the field distribution inside heavily irradiated sensors using Edge-TCT [11] to extract the velocity profiles. This can help developing a precise method for predicting the electrical performances of the devices up to the maximum fluence anticipated for a given experiment and to accurately predict expected performances of heavily irradiated sensors in term of signal, noise and evolution after irradiation.

\section{Intelligent Trackers}

As the luminosity of accelerators increases it becomes increasing challenging to maintain the present level of physics performance of the detectors and the low trigger thresholds necessary for studying the Higgs boson. Therefore new triggering and data collection approaches are required to improve filtering and data throughput. Several approaches are under considerations.

One option aims at reducing the volume of tracker data to be read out with the definition of a Region-of-Interest (RoI) set by the calorimeter and muon Level-0 triggers. This option can only be implemented in a split Level-0/Level-1 trigger architecture. This is the baseline design for the ATLAS L1 track trigger for the HL-LHC [1]. The RoI-driven L1Track trigger would provide all tracks in a number of relatively small regions of the inner tracking detector. The full data from the tracker cannot be read out at the full beam-crossing rate or even at a Level-0-Accept rate of $500 \mathrm{kHz}$. The regional readout design of the track trigger addresses this problem by starting from the Level-0 trigger RoIs and then reading only a subset of the tracker data. Conceptually, this is similar to the current design of the ATLAS Level-2 trigger, FTK [12], except that between the Level-0-Accept and the Level-1 decision the data are buffered on detector in the inner detector readout electronics. One of the main advantages of the RoI-driven L1Track design is that it has little impact on the inner detector layout that, apart from the depth of the front-end buffers, can be optimized almost independently from the design of the trigger. The main challenge for the RoI-driven L1Track trigger is to fit within the ATLAS Phase II trigger latency constraints. 
A more challenging concept relies on finding track segments that would be used as primitives for a self seeded track trigger $[13,14,15]$. This requires specially designed and instrumented trigger layer pairs that would be used to identify high transverse momentum (high- $\mathrm{p}_{\mathrm{T}}$ ) candidate track segments with low latency to seed the first level track trigger with candidate collisions for full readout. The rejection of low $\mathrm{P}_{\mathrm{T}}$ tracks relies on correlating signals in two closely spaced sensors since the distance between the hits in the $\mathrm{x}-\mathrm{y}$ plane is correlated with the curvature of the track

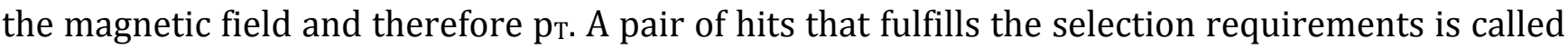
a "stub". This method places constraints on the geometrical layout of the tracker. For a given $\mathrm{p}_{\mathrm{T}}$, the distance between the hits forming the stub is larger at larger radii. If the module is placed in end-cap configuration, the same discriminating power is obtained with a larger spacing between the two sensors, compared to a barrel module placed at the same radius. CMS is currently considering a long barrel and a barrel plus end-cap layout. . In order to implement isolation cuts in the Level-1 selection, the Tracker will have to provide information on all tracks above $2 \mathrm{GeV}$, with a precision in the position of the collision vertex along the beam axis of at least $1 \mathrm{~mm}$.

Two different architectures for the CMS L1 track reconstruction are also considered. One using hits with a small cluster size and pattern recognition with a content addressable memory (Associative Memory, AM) which compare the hits with pre-stored track patterns. Research and development is necessary to develop new generations of CAM chip regardless of whether such a chip will be used for efforts such as an FTK upgrade or for the L1Track pattern recognition [16].

In a second implementation that favors a long barrel geometry, the transverse momentum of charged particles is determined from the correlation between stacked double strip layers. The stacked trackers are composed by one or more double pixel layers, spaced approximately one millimeter apart. A pair of stacks spaced a few centimeters apart is called a "double stack". When hits are registered on both stack members within a stack and the separation in of these hits is consistent with a high-p т $_{\text {T }}$ particle, the set of hits become known as a "stub", the basic trigger primitive of a stacked-tracker geometry. Correlated stubs on two or more stacks are called a "tracklet". Pattern-based hit correlation of properly built clusters of hits provide L1 trigger primitives to the hardware trigger. These can be combined together in a projective geometry to perform a rough tracking to be implemented online, returning rough $\mathrm{p}_{\mathrm{T}}$, direction, and vertex information for a candidate track. The modules required implementing this approach benefit by using 3D connectivity, which incorporate through-silicon-vias to provide correlation between the tiers to find stubs [3].

In general $R \& D$ is needed to improve the link technology and transfer large amount of data in shorter times. Recently the first prototype of a $60 \mathrm{GHz}$ wireless Multi-gigabit data transfer topology is currently under development at University of Heidelberg using IBM $130 \mathrm{~nm}$ SiGe HBT BiCMOS technology. This could delivering multi-gigabit per second data rates, and therefore it could be a potential solution to the challenging data transfer rate from highly granular tracking detectors. 


\section{Summary}

Even if the requirements on the ATLAS and CMS strip trackers are not as severe as for the pixel detectors, there are many issues that remain very challenging. Furthermore since the trackers for the CMS and ATLAS upgrade will cover large areas, special attention must be places on integration, powering, cooling, and material support. The R\&D areas are summarized in Table I.

Table I: Requirement for silicon microstrip detectors for the HL-LHC upgrade

\begin{tabular}{|c|c|c|c|c|c|c|}
\hline Issues & System & Sensors & FEE & $\begin{array}{l}\text { Optical } \\
\text { links }\end{array}$ & $\begin{array}{l}\text { Mechanics \& } \\
\text { Cooling }\end{array}$ & Powering \\
\hline $\begin{array}{l}\text { Radiations } \\
\text { Hardness }\end{array}$ & & $\begin{array}{l}2 \mathrm{E} 15 \\
\mathrm{n} / \mathrm{cm}^{2}\end{array}$ & $130 \mathrm{~nm}$ & $65 \mathrm{~nm}$ & $\begin{array}{l}\text { Certify } \\
\text { materials }\end{array}$ & $\begin{array}{l}\text { DC-DC / } \\
\text { serial } \\
\text { power }\end{array}$ \\
\hline $\begin{array}{l}\text { Reduce \% } \\
\text { radiation } \\
\text { Length }\end{array}$ & & $\begin{array}{l}\text { Low cost } \\
\text { thinner } \\
\text { sensors } \\
250 \mu \mathrm{m}\end{array}$ & $\begin{array}{l}\text { Reduce } \\
\text { power/ } \\
\text { channel }\end{array}$ & $\begin{array}{l}\text { Low } \\
\text { power } \\
\text { links }\end{array}$ & $\begin{array}{l}\text { Low mass } \\
\text { support } \\
\text { material } \\
\text { CO2 cooling }\end{array}$ & $\begin{array}{l}\text { Low mass } \\
\text { component } \\
\mathrm{s}\end{array}$ \\
\hline Occupancy & $1 \%$ & $\begin{array}{l}\text { Reduce } \\
\text { Strip } \\
\text { length/ } \\
\text { introduce } \\
\text { micropixels }\end{array}$ & & $\begin{array}{l}\text { High } \\
\text { bandwidth }\end{array}$ & & \\
\hline Resolution & & $\begin{array}{l}\text { Pitch } 50 \\
\mu \mathrm{m}\end{array}$ & & & Alignment & \\
\hline Connectivity & & C4 bonding & & & & \\
\hline $\begin{array}{l}\text { Tracker } \\
\text { information } \\
\text { for level } 1\end{array}$ & & $\begin{array}{l}\text { Edgeless, } \\
\text { Slim edge }\end{array}$ & & $\begin{array}{l}\text { Increase } \\
\text { bandwidth }\end{array}$ & $\begin{array}{l}\text { TSV } \\
\text { 3D electronics }\end{array}$ & \\
\hline Integration & $\begin{array}{l}\text { Facility } \\
\text { to } \\
\text { build } \\
\text { and } \\
\text { access }\end{array}$ & $\begin{array}{l}\text { Reduce } \\
\text { type of } \\
\text { sensors }\end{array}$ & & & $\begin{array}{l}\text { Reduce } \\
\text { Module types }\end{array}$ & \\
\hline
\end{tabular}




\section{References}

[1] Letter of Intent for the Phase-II Upgrade of the ATLAS Experiment, CERN-2012-022, LHCC-I023, December 2012.

[2] S. Abbaneo and A. Marchioro, A hybrid module architecture for a prompt momentum discriminating tracker at HL-LHC, JINST 7 C09001 (2012). D. Abbaneo, Upgrade of the CMS tracker with tracking trigger, JINST 6 C12065 (2011)

[3] R. Lipton, 3D technology for intelligent trackers, JINST 5 C10006 (2010).

[4] D. Braga, G. Hall, L. Jones, P. Murray, M. Pesaresi, M. Prydderch and M. Raymond, CBC2: $a$ microstrip readout ASIC with coincidence logic for trigger primitives at HL-LHC, JINST 7 C10003 (2012).

[5] W. Dabrowski et al., Design and performance of the ABCN-25 readout chip for ATLAS Inner Detector Upgrade, IEEE Nucl. Sci. Symp. Conf. Rec. (2009) 373.

[6] P. Moreira et al., The GBT Ser-Des ASIC prototype , JINST 5 C1102 (2010)

[7] M. Bochenek and W. Dabrowski, Prototype linear voltage regulators for the ABC130 front-end chip for the ATLAS Inner Tracker Upgrade, JINST 8 C01037 (2013)

[8] L. Feld, M. Fleck, M. Friedrichs, R. Hensch, W. Karpinski, K. Klein,1 J. Sammet and M. Wlochal, $A D C$-DC conversion powering scheme for the CMS Phase-1 pixel upgrade, JINST 8 C02024 (2013)

[9] Satish Dhawan and Richard Sumner, Detector Powering in the 21st Century Why stay stuck with the Good old 20th Century methods, Physics Procedia 37 ( 2012 ) 181 - 189

[10] A. Dierlamm, Silicon sensor developments for the CMS Tracker upgrade, JINST 7 C01110 (2012)

[11] Gregor Kramberger, V. Cindroa I. Mandic, M. Mikuž, M. Milovanovic, M. Zavrtanik, Electric field modeling in heavily irradiated silicon detectors based on Edge-TCT measurements, POS (VERTEX 2012), 022.

[12] ATLAS Collaboration. FTK: a hardware track finder for the ATLAS trigger Technical Proposal, 2010. ATL-D-ES-0036; https://edms.cern.ch/document/1064724/1.0.

[13] G Boudoul, A Level-1 Tracking Trigger for the CMS upgrade using stacked silicon strip detectors and advanced pattern technologies, JINST 8 C01024 (2013).

[14] A Schöning, A self seeded first level track trigger for ATLAS, JINST 7 C10010 (2012).

[15] E. Salvati, A level-1 track trigger for CMS with double stack detectors and long barrel approach, JINST 7 C08005 (2012).

[16] G. Deputch et al. Development of 3D Vertically Integrated Pattern Recognition Associative Memory, (VIPRAM). FERMILAB-TM-2493-CMS-E-PPD-TD , 2011.

[17] H K Soltveit, R Brenner, A Schöning and D Wiedner, Multi-gigabit wireless data transfer. 


\section{Next Generation Strip Detectors for the Intensity Frontier Contribution of Marina Artuso (Syracuse University)}

Silicon strip sensors are now the standard building block of charged particle tracking detectors in several different applications including Intensity Frontier experiments. We will summarize the purpose, goals, and challenges of the next generation of such devices being currently designed.

Silicon microstrip detectors for "intensity frontier" applications must meet requirements that are similar in nature, although quantitatively different, than the ones planned for "high energy frontier" applications. Two different implementations are discussed here, one being planned for the LHCb upgrade and the other for Belle II. The main requirements are summarized in Table I.

Table I: Requirements for silicon microstrip detectors for the UT Tracking Module in the LHCb upgrade and for the SVD of Belle II

\begin{tabular}{|l|l|l|}
\hline Requirement & LHCb UT [1] & Belle II SVD [2] \\
\hline $\begin{array}{l}\text { Radiation fluence } \\
\text { expected }\end{array}$ & $2 \times 10^{14} \mathrm{n}_{\mathrm{eq}} / \mathrm{cm}^{2}$ & \\
\hline technology & $\begin{array}{l}\text { Single sided n-in-p or p-in- } \\
\mathrm{n}\end{array}$ & Double-sided \\
\hline pitch & $100-200 \mathrm{~mm}$ & \\
\hline Strip length & $5-10 \mathrm{~cm}$ & Double metal layer \\
\hline Fan-out & Double metal layer or & $50 \mathrm{~ns}$ \\
\hline $\begin{array}{l}\text { Front end electronics } \\
\text { timalog section peaking }\end{array}$ & $25 \mathrm{~ns}$ & $\begin{array}{l}\text { Analog pipeline non-zero } \\
\text { suppressed }\end{array}$ \\
\hline Front end readout scheme & $\begin{array}{l}\text { Zero suppressed digital 3- } \\
5 \text { bit ADC }\end{array}$ & $0.58 \% \mathrm{X}_{0} /$ layer \\
\hline Average radiation length & $<1 \% \mathrm{X}_{0} /$ layer & $0.35 \mathrm{~W} / 128$ channels \\
\hline Power dissipation & & \\
\hline
\end{tabular}


Although the experimental environments in which these two detector operate are different, similar requirements need to be addressed:

1. The Belle II detector, in order to maximize the spatial information achievable with a single silicon layer, utilizes double sided readout to extract more information from a single $\mathrm{Si}$ layer.

2. The LHCb design is including a "shaped inner edge," similar to the VELO design, to achieve closer proximity to the beam pipe [3].

3. Radiation resistance considerations are relevant to both detector systems, although they are much more demanding in a hadron machine. In the case of the LHCb upgrade it is important to withstand highly non-uniform irradiation. In addition, as fan-out of the signal connections may be implemented through a double layer of metallization, possible radiation effects induced by this layer need to be established [4].

4. A minimization of the overall material budget is critical to optimize tracking efficiency and resolution. This can be achieved only with a design that tightly integrates the electrical, cooling, and mechanical specifications.

5. In order to cope with the higher occupancy (Belle II) and to achieve the necessary data acquisition speed (LHCb), fast shaping times are needed.

6. The requirement of pushing the data out of the detector at $40 \mathrm{MHz}$ implies that the $\mathrm{LHCb}$ tracking system needs to implement the zero suppression algorithm in the front end ASIC.

Major R\&D items:

1. Front end electronics for fast data acquisition system

2. Low mass system design (synergistic with the chapter on low mass mechanics and cooling)

3. Low mass interconnection scheme preserving fast data rate

4. Qualification of all the components for the necessary level of radiation resistance.

\section{References}

[1] Letter of Intent for the LHCb Upgrade, CERN-LHCC-2011-001 (20122)

[2] Belle II Technical Design Report, KEK-Report-2010-1

[3] The LHCb Detector at LHC, Journal of Instrumentation 3 S08005 (2008) doi:10.1088/17480221/3/08/S08005

[4] Radiation damage in the LHCb Vertex Locator, Affolder, A (Liverpool U.) et al. arXiv:1302.5259 (2013) 


\section{Low Mass Support and Cooling for Future Collider Tracking Detectors}

\section{William Cooper (Fermilab), Carl Haber (LBNL), David Lynn (Brookhaven National Lab)}

\section{Introduction:}

Future high luminosity (HL) and high energy (HE) colliders present a variety of technical challenges for the development of low mass support structures and cooling systems. These challenges derive from the need to operate large area sensor arrays with high channel counts, at low temperature, and in high radiation fields.

Physics reach is being extended in both HE and HL colliders due to increased cross section and interaction rates. These both lead to increased particle multiplicity. As a result the innermost tracking detectors, typically silicon but potentially diamond or other materials, are subject to unprecedented radiation dose exposures. To decrease detector leakage currents (and hence electronic noise), and stabilize depletion voltage changes, these detectors will need to operate at ever decreasing temperatures. Increased multiplicity also results in the need for finer detector segmentation and thus higher readout electronics density. Therefore future detectors will have greater power densities that will require efficient cooling. Table 1 lists typical operating or environmental conditions for high luminosity tracking detectors.

\begin{tabular}{|l|l|}
\hline \multicolumn{2}{|l|}{ Operating/Environmental Conditions } \\
\hline Max Operating Temperatures & $40^{\circ} \mathrm{C}$ \\
\hline Min Operating Temperatures & $-50^{\circ} \mathrm{C}$ \\
\hline Ionizing Radiation TID & $50-1000 \mathrm{Mrad}$ \\
\hline Non-ionizing radiation Levels & $1-10 \cdot 10^{15} \mathrm{n}_{\mathrm{eq}} / \mathrm{cm}^{2}$ \\
\hline Magnetic Fields & $>2 \mathrm{~T}$ \\
\hline Typical Sensor Area & $100 \mathrm{~m}{ }^{2}$ \\
\hline Typical Power Densities & $\sim 100 \mathrm{~mW} / \mathrm{cm}^{2}$ \\
\hline Total Power & $100 \mathrm{KW}$ \\
\hline
\end{tabular}

Table 1: The typical environmental and operating conditions for HE and HL collider tracking detectors. 
The size and scale of the detectors at HL and HE must be large to generate enough hit points, over sufficient lever arm, to permit good track and momentum reconstruction. Typical tracking detector sizes can exceed $100 \mathrm{~m}^{2}$ of silicon. The mechanical support must therefore be similar in scale, and consequently must efficiently utilize the support material to reduce overall detector radiation length. This serves to reduce multiple scattering and conversions. Efficient material use requires structures to perform dual (or triple) roles of providing support, cooling, and/or electronic functions such as shielding and signal/power distribution. Use of low-Z materials that have a high stiffness to mass ratio is required. Table 2 lists general requirements for support structures for HL and HE tracking detector supports.

\begin{tabular}{|l|}
\hline Requirements \\
\hline Long radiation length materials \\
\hline High stiffness/mass ratio (i.e. stability) \\
\hline Radiation tolerant materials \\
\hline Dual (or triple) purpose elements \\
\hline Large scale manufacturability \\
\hline Minimal thermal and humidity effects \\
\hline
\end{tabular}

Table 2: General requirements for mechanical supports for HL and HE collider tracking detectors.

\section{Materials:}

In general, low-Z materials are to be preferred for use in support structures as these will typically have longer radiation lengths. Polymers such as plastics have long radiation lengths as well. A material is usually selected for its stiffness and/or thermal conductivity. All materials need to be qualified as radiation hard to levels somewhat higher than the total radiation dose expected over the lifetime of the detector.

Table 3 lists some materials used in tracking detectors for mechanical support and cooling. Their relative merits in terms of stiffness, thermally conductivity, and radiation length are indicated with " $\checkmark$ "s. Generally metals are to be avoided relative to other materials as their performance, with the exception of beryllium, is lower. (Beryllium suffers from high cost and safety issues that limit its use). Materials derived from carbon have found wide use for their overall favorable properties and versatility. Ceramics may be used in limited quantities when simultaneously electrical isolation and high thermal conductivity is desired. Polymers find application when 
complex shapes are needed (either via machining or injection molding) and stiffness is not the primary driver.

\begin{tabular}{|l|l|l|l|}
\hline & Structural (stiff) & Thermally Conductive & Low (long) Radiation Length \\
\hline Carbon based materials & & & \\
\hline Carbon Foam & $\checkmark$ (as core material) & $\checkmark \checkmark$ & $\checkmark \checkmark \checkmark$ \\
\hline Carbon Fiber Reinforced Plastic & $\checkmark \checkmark \checkmark$ & $\checkmark \checkmark \checkmark$ & $\checkmark \checkmark$ \\
\hline Carbon Honeycomb & $\checkmark \checkmark$ (as core material) & X & $\checkmark \checkmark \checkmark$ \\
\hline TGP & X & $\checkmark \checkmark \checkmark$ & $\checkmark \checkmark$ \\
\hline Polymers/Plastics & & & \\
\hline Peek & $\checkmark$ & X & $\checkmark \checkmark$ \\
\hline Liquid Crystal Polymer & $\checkmark$ & X & $\checkmark \checkmark$ \\
\hline Ceramics & & & \\
\hline Alumina & $\checkmark \checkmark \checkmark$ & $\checkmark$ & $\checkmark$ \\
\hline Beryllia & $\checkmark \checkmark \checkmark$ & $\checkmark \checkmark \checkmark$ & $\checkmark \checkmark$ \\
\hline Aluminum Nitride & $\checkmark \checkmark \checkmark$ & $\checkmark \checkmark \checkmark$ & $\checkmark$ \\
\hline Metals & & & \\
\hline Beryllium & $\checkmark \checkmark \checkmark$ & $\checkmark \checkmark \checkmark$ & $\checkmark \checkmark$ \\
\hline Aluminum & $\checkmark \checkmark$ & $\checkmark \checkmark \checkmark$ & $\checkmark$ \\
\hline Titanium & $\checkmark \checkmark$ & $\checkmark$ & X \\
\hline Stainless Steel & $\checkmark \checkmark$ & $\checkmark$ & \\
\hline
\end{tabular}

Table 3: Typical materials and their relative performance in terms of stiffness, thermal conductivity, and radiation length.

\begin{tabular}{|l|l|}
\hline$\checkmark \checkmark \checkmark$ & excellent \\
\hline$\checkmark \checkmark$ & good \\
\hline$\checkmark$ & decent \\
\hline$X$ & poor \\
\hline
\end{tabular}

\section{Structures}

With the focus mainly, and naturally, on carbon based composite materials, a great variety of structures and arrangements have already been studied, and many more may be envisioned for the future. Figure 1 shows a prototypical support and cooling structure fabricated from predominately carbon based materials. This particular structure targets a two layer pixel tracking system for a HL collider. In application, a group of these would surround the interaction region. The structure is constructed as an I-beam for rigidity. Note the structure's multi-purpose functions. It supports two radii of (pixel) sensors while providing cooling to each. Carbon fiber re-enforced plastic facesheets provide stiffness and thermal conductivity. Carbon foam provides a thermally conductive path from the metal pipes to the facesheets. 


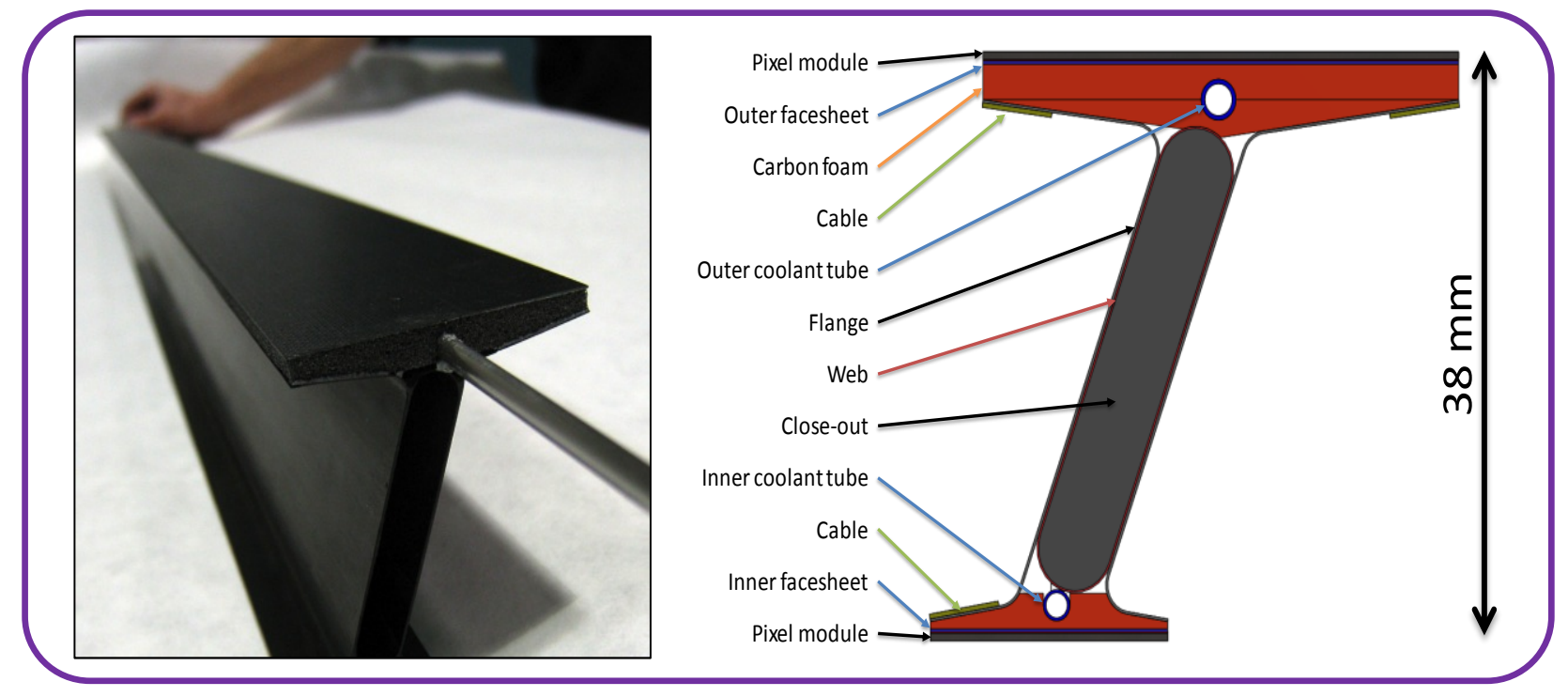

Figure 4. Carbon composite structure that supports and cools two radii of pixel detectors.

This structure includes many of the generic features of future configurations; these include embedded cooling, high thermal conductivity inserts, engineered materials, and integrated electrical services.

\section{R\&D Directions}

There are a variety of $R \& D$ directions which could have significant impact on the thermal, mechanical, and electrical performance of support and cooling systems.

- Multiple Functions: Future R\&D will continue to have mechanical structures perform multiple functions. For example there is ongoing work to make use of the electrical conductivity of carbon fiber facesheets to provide electrical shielding of sensors from high frequency clock signals as well as from fields generated from DC-DC converters. R\&D in this area would focus, for example, on material properties, co-curing, and new ways to integrate materials and services together. Figure 2 shows and example from current R\&D.

- Improved materials: This is a broad topic, central to the undertaking. It covers novel materials such as new ceramics, new carbon or other types of low density foams, adhesives, and high modulus fibers, among others.

- Engineered composites: The combination of existing and new materials into composite structures has already been fruitful. This R\&D extends that line to new arrangements and configurations which could yield improved, or even novel, properties. Examples are laminate orientations and mixed materials.

- Materials science and nanotechnology: The materials science and nanotechnology community may offer approaches and materials which could have significant impact on R\&D for future 
trackers. For example, recent work has indicated that very small percentage dispersions of nanomaterials in polymers can have dramatic effects on thermal conductivity and strength. This may lead to improved adhesives, and matrices for composite pre-pregs. Such preparations, and the processes need to create them, are state-of-the-art. It is important that our community engage where possible with chemists and materials scientists to further these developments.

- Spin-offs: As in the past, other fields may benefit from developments originating in HEP. Collaboration, in particular with the Basic Energy Sciences, Nuclear Physics, and Accelerator communities should be encouraged. New ideas can emerge from this interplay. Additional funding sources can sustain R\&D capabilities through the inevitable funding cycles.

- Measurement techniques and QA: Characterization of materials and structures is also key to understanding their performance both in R\&D and through fabrication. New and emerging tools should be considered, studied, and evaluated. Among these are 3D metrological methods, acoustical microscopy, large area automated inspection, thermal imaging, and precision survey, both static and dynamic.

- Simulation and Modeling: Analytical and numerical models of materials and composites are key tools in the development process. Recent work on finite element analysis of low density carbon foam has led to a predictive understanding of the dependence of thermal conductivity on density. Engagement with industrial and academic collaborators in this area should be encouraged.

- 3D printing: This is an emerging area which has received considerable attention. It has already been applied to create prototypical structures in a number of HEP applications. It should be watch as it develops further and in particular as new materials became available as printing media.

- Materials Database: Identifying and specifying materials of interest, and their properties, can sometimes be difficult to coordinate. For design, modeling, and simulation, it is important that workers use common and agreed upon values. A public access database of materials properties should be established. The database would include physical, structural, thermal, and radiation hardness properties. As time progresses, the database could be extended to include specific processes and techniques to match materials to low-mass detector requirements.

During the next ten years and beyond, a cooperative effort by industry, academic institutions, and national laboratories is essential to develop the materials, structures, tools, fabrication techniques, and thermal mechanical systems that will be required in next-generation and 
future detectors. Industrial involvement helps ensure that the latest materials and fabrication techniques are known and will be cost effective in appropriate quantities. Involvement of academic institutions and national laboratories is essential to monitor industrial developments for their applicability to physics needs, encourage crucial developments, and develop and test state-of-the-art new materials and processes. A cooperative effort among the three research and development communities helps minimize duplication of facilities and effort, thereby allowing development to proceed in a timely and cost-effective manner.

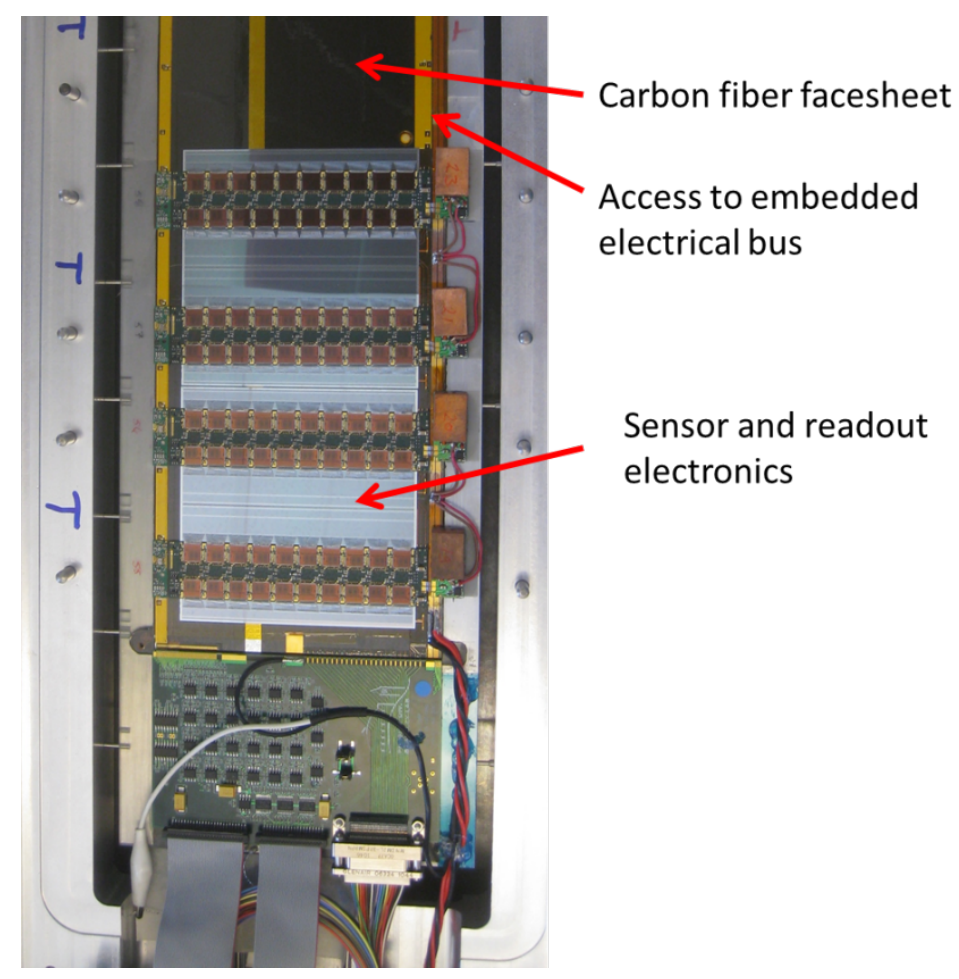

Figure 2. A current multiple function example. Sensor and readout electronics is mounted on structural laminate of carbon fiber facesheets, an embedded electrical bus cable, carbon foam and honeycomb core, and coolant carrying channels. The top face-sheet serves also as an electrical shield layer. 


\title{
Development of Large-Area Photosensors for Future HEP Experiments
}

\author{
M. Wetstein (UChicago/ANL), J. Va'vra (SLAC), M. C. Sanchez \\ (Iowa State/ANL)
}

\section{Introduction}

Photosensors provide essential functionality for HEP experiments in a wide variety of contexts. Increasingly, the demands of HEP experiments are pushing beyond standard capabilities of conventional phototubes. In this whitepaper, we examine the experimental needs of future HEP Experiments and how advances in photodetector technologies might impact their physics reach.

Photosensors are typically used in three broad categories of detector systems: (1) Large Cherenkov arrays and water Cherenkov tanks for full event reconstruction in neutrino experiments, proton decay, and detecting atmospheric showers from cosmic rays; (2) Ring Imaging Cherenkov (RICH) and Detectors of Internally Reflected Cherenkov light (DIRC) detectors used for particle identification in heavy flavor collider experiments; (3) and simple time-of-flight (TOF) systems used for vertex reconstruction and time-of-flight mass spectroscopy in colliders. The demands on these photosensors vary widely from one experiment to the next, but can be divided between the two main types of operational conditions:

1) Low-rate environments such as neutrino, double beta decay, and proton decay experiments. Under these conditions low-cost coverage, improved resolution for event reconstruction, and single photon counting capabilities are most important for background rejection, signal selection efficiency, tracking, and momentum resolution.

2) High-rate environments typical of collider based experiments. Here photodetectors need to be robust over years of operation under intense radiation, and capable of handling high event rates per unit area per unit time without saturating. Readout electronics must be designed to handle the high rates and track multiplicities.

Common to both experimental categories is the need to instrument large areas economically and the need for improvements in the combined timing and imaging resolutions.

\section{Physics Needs and Desired Detector Capabilities}

Here we describe some of the areas ripe for improvements in photosensor technology and provide a few examples of physics issues to be addressed by these developments.

\section{Cost per Area Coverage and Light Collection Efficiency}


Given the large scales of future collider (eg Atlas and CMS) and neutrino experiments (approaching megatons), a substantial improvement in the cost-per-area coverage of photodetectors can have a positive impact on large experiments. While this is not the only factor, improvements that allow better light collection and area coverage per unit cost can address key physics needs within the currently tight budgetary environment. For physics processes with low light yields, such as the detection of supernova neutrinos in large water Cherenkov detectors, better photon statistics translate directly to better energy resolution and improved sensitivity to the $\mathrm{MeV}$-scale charged particles near the Cherenkov threshold [1]. The Daya Bay II collaboration provides another interesting example where light collection would enable new physics reach. In this experiment, a full spectrum analysis of the oscillation signal at many energies over the $60 \mathrm{~km}$ baseline would allow discrimination between the normal and inverted neutrino mass hierarchies. Thus the detectors for Daya Bay II would require excellent energy resolutions: 3 $\mathrm{MeV} / \mathrm{sqrt}(\mathrm{E})$ compared with $8 \mathrm{MeV} / \mathrm{sqrt}(\mathrm{E})$ in the comparable Kamland detector [2]. In the case of heavy flavor physics, the ability to replace multiple MCP-photosensors with single, large-area MCPs might also lower costs without compromising either time or imaging resolution.

For the same area coverage, light collection can be improved by advances in photocathode quantum efficiency. Not only the average quantum efficiency, but the spectral response of photocathodes is also of interest. Further gains can be made using light collectors, devices such as Winston Cones and wavelength shifting plastics. These allow for improved light collection with the same number of photosensors. Finally, in the context of large water Cherenkov experiments, it may be possible to employ full imaging optics such as parabolic and spherical reflective geometries. These approaches trade some angular phase space for increased area coverage. With the addition of precision timing to the spatial image, it may be possible to build a complete image, along with corrections for near field optical distortions from non-parallel incident light.

\section{Imaging Capabilities}

Conventional PMTs are single pixel detectors. Position of the incident photons cannot be resolved except insofar as they hit a particular PMT. Photodetectors with spatial granularity would bring needed capabilities to HEP experiments. For example, such photosensors can be employed in the context of imaging optics, where the pattern of Cherenkov light is mapped to a smaller surface area by reflectors. In addition, Cherenkov- and scintillation-based neutrino detectors can make better use of the fiducial volume near the edge of the detector is it is possible to resolve photon positions within single photosensors. These capabilities are also important in RICH and DIRC systems, where the geometry and time-of-flight of the Cherenkov light is used for particle identification.

\section{Precision Timing and Imaging Capabilities}

Many measurements in particle physics are limited by the time resolution of particle detectors. Timeof-flight systems approaching few picosecond resolutions would allow mass-spectroscopy and thus efficient flavor identification of high energy jets in collider experiments. 
In many cases, the combination of precision timing and spatial resolutions is as important as the timing itself, allowing the measurement of event kinematics in terms of 4-vectors rather than 3-vectors. Measurements of the relative arrival times of particles with resolutions approaching single picoseconds over a drift distance of $1.5 \mathrm{~m}$ could be combined with track information to determine particle masses and thus the quark composition of hadronic jets. Timing measurements can also be used to match high energy gammas to their respective interaction vertices, providing a useful capability for collider experiments interested in measuring certain Higgs, KK, and gauge mediated SUSY signatures [3].

Similarly, in neutrino experiments where Cherenkov light is present, the combination of precision position and time information can be used to reconstruct tracks and EM showers with high resolution, which is important in reducing backgrounds for electron neutrino selection, as well as discrimination between gammas and electrons [4].

Heavy flavor physics experiments rely on technologies such as RICH (Ring Imaging CHerenkov) and DIRC (Direct Internally Reflected Cherenkov) detectors, where the geometric and timing properties of Cherenkov light emitted by high energy particles passing through thin radiators are used to separate between particles of similar momentum but differing mass. DIRC detectors, in particular, rely on timing to correct for chromatic dispersion effects on these Cherenkov rings. In all of these cases the ability to accurately measure both the positions and times of each arriving photon is very important [58].

\section{Single Photon Counting}

In neutrino experiments, energy is largely measured by light yield. Accurate counting of individual photons in Cherenkov detectors is a necessary ingredient. Identification of single photons can be greatly improved by the combination of narrow pulse height distribution with fine-grained spatial and time separation. In low rate contexts, the ability to separate between photons in space and time enables essentially digital photon counting. In addition, this capability also enables improved separation between direct light and scattered light, prompt Cherenkov and scintillation light, and photons from multiple tracks. Precision photon counting could also improve the sensitivity of large Water Cherenkov detectors to low energy charged particles, close to the Cherenkov emission threshold, where observing small numbers of emitted photons above dark noise becomes challenging.

\section{Noise and Radiopurity}

Photosensors are used in a variety of low-rate experiments. For deep underground neutrino experiments, dark rates from spontaneous firing of photodetectors -typically from the photocathodereduce the signal-to-noise from Cherenkov and scintillation light [9]. The combination of coincidence triggers and time vetoes (in the case of beam neutrinos) can greatly ameliorate this effect. However, for rare, non-accelerator events with low light yields, dark rates can present a problem. The ability to make photosensors from very radio-pure materials is also an important capability for keeping backgrounds down in dark matter and double beta decay experiments [10]. 


\section{Robustness}

Many HEP experiments subject photosensors to extreme conditions, particularly low temperatures, high pressures, and high radiation environments. Continued work on the implosion mechanisms stresstesting and containment are important for vacuum photosensors in deep water and ice applications. It is also important that photosensors can survive exposures to cryogenic temperatures such as those of liquid argon. Even functional aspects of the sensor, such as the resistivity of the photocathode and gain stages can depend on temperature and must be optimized for low-temperature operation.

\section{Radiation Hardness}

In most collider-based environments, photosensors will be subject to unprecedented radiation doses. In Belle-II, detectors will see luminosities of $10^{36} \mathrm{~cm}^{2} \mathrm{sec}^{1}$, with neutron doses up to $\sim 10^{11}-10^{12} / \mathrm{cm}^{2}$, charged particle doses of $\sim 5 \times 10^{11} / \mathrm{cm}^{2}$, and $\sim 5 \times 10^{11} / \mathrm{cm}^{2}$ in just 10 years. In the central region of the Atlas Detector at 1 meter from the interaction point, a detector will see gammas and charged particles at a rate of $\sim 10^{5} / \mathrm{cm}^{2}$, and neutrons at $\sim 10^{6} / \mathrm{cm}^{2}$. These conditions require an R\&D to develop radiation hard materials, such as fused silca and optical glues for DIRC-based detectors, SiPMT-based detectors with a good resistance to large neutron doses, photocathodes with a resistance to the radiation (a choice between bialkali and multi-alkali), electronics and many other materials. For example, SiPMTs develop a large noise rate and the pulse height resolution damage after a neutron dose of $\sim 10^{10} / \mathrm{cm}^{2}$. [11]

\section{Rate Handling and Dynamic Range}

Many high-rate experiments test the rate handling limits of photosensors. At RHIC and the LHC, the Star and Alice Experiments will see track multiplicities of 10,000 per event at a rate of $1 \mathrm{kHz}$. The TORCH Experiment in $\mathrm{LHCb}$ will see a rate of roughly $10 \mathrm{MHz} / \mathrm{cm} 2$ and $10 \mathrm{e} 11$ photons per quartz detector per second [12], [13]. Optimizing photodetectors to operate at high rates without saturation or drop in gain is very important. In many contexts, particularly those of atmospheric Cherenkov arrays, photosensors must also be able to handle very wide ranges in light intensity [13].

\section{Recent and Ongoing Efforts}

\section{Work with Conventional PMTs}

The performance of large-area conventional phototubes has been studied in detail by a diversity of neutrino of potential experiments using large, monolithic, optical detectors [14 - 17].

As an example, recent design work by the Long Baseline Neutrino Experiment (LBNE) collaboration [14] targeting a 200-kton Water Cherenkov detector, resulted in the selection of the 12-inch Hamamatsu R11780 PMT. This is a 12" PMT available with peak quantum efficiency of $32 \%$ at 390 $\mathrm{nm}$. The investigation of these tubes showed that they are good candidates for large optical detectors, due not only to their excellent performance but also a mechanical construction that can withstand high water pressures [11]. The performance measurements done showed peak-to-valley ratios greater than 2 , transit time spreads around $1.3 \mathrm{~ns}$ and late-pulsing probabilities of less than 5\% [11]. While the LBNE 
large Water Cherenkov design using 45,000 of these will not be going forward, the R\&D is relevant to other possible optical detectors.

Similarly the HyperK working group [16] is working on the selection of phototubes for a 0.56 megaton fiducial Water Cherenkov detector, 10 times larger than the Super-Kamiokande detector. A fundamental requirement of this experiment is to be sensitive to a broad range of energies: from low $\mathrm{MeV}$ energies for supernova and solar neutrinos to atmospheric neutrinos in the GeV range. The baseline candidates for the Hyper-Kamiokande experiments are 99,000 large 20-inch PMT similar to those used in Super-Kamiokande (Hamamatsu R3600). Each of this would need to be surrounded by a protective case to avoid any accident cause by a chain-reaction starting from one phototube implosion. Other possibilities are of course being considered a 8 or 10-inch tube with a quantum efficiency of $40 \%$ which is almost double that of the R3600, the number of phototubes required would 1.4 times the number required for $20 \%$ coverage for the 20 -inch models. Finally development is on-going on hybrid photodetectors (HPDs) which uses a an avalanche photodiode instead of metal dynodes. These photodetectors provide high performance in timing and gain uniformity as well as fast response and better signal to noise ratio. Transit time spread for $1 \mathrm{PE}$ have been measured to be approximately $0.6 \mathrm{~ns}$ for the 8-inch version and predicted to be $1.1 \mathrm{~ns}$ for the 20 -inch version. These devices also demonstrate excellent photon separation with a peak to valley ratio of 8.9 compared to 1.9 in the conventional PMTs. The dark current in these devices might however be limiting for low energy physics in large water detectors.

Other collaborations such as LENA [15], Icecube [17], SNO [18] and MiniBoone [19] have done similarly detailed characterization studies of currently available photodetectors.

Work has also been done in designing elements that would enhance light collection efficiency such as Winston cones, wavelength shifting discs, and lensing systems. Such approaches can increase light collection up to $20-25 \%$, usually at the cost of effective fiducial volume due to limited angles of acceptance, or decreased timing resolution [16], [20].

\section{LAPPD Collaboration}

The Large Area Picosecond Photodetector (LAPPD) has developed flat-panel, large-area (8"x8") MCPdetectors for use in a variety of HEP contexts. These detectors consist of 8 " $\mathrm{x} 8$ " square MCPs made by thin-layer deposition of enhanced secondary electron emissive materials on substrates made from drawn glass capillary tubes. The vacuum package is based on flat-panel, sealed-glass technology, with no external pins. A single high voltage connection is made to the top window, which extends out of the vacuum region of the glass envelope. Operational voltages are set by a series of resistive spacers, and DC current through the MCP stack exits through the same stripline anode as the fast signal from MCP pulses. The delay-line, anode pattern consists of silver micro-striplines, silk-screened onto the bottom glass plate, and a frit-base vacuum seal is made over those striplines, between the detector wall and the bottom plate. The nominal LAPPD design addresses the entire detector system, including front-end electronics. The LAPPD collaboration has developed low-power ASICS, capable of sampling fast pulse 
shapes with $1.5 \mathrm{GHz}$ analog bandwidth and up to 15 Msamples/sec sampling rates. Preliminary funding for this commercialization of the LAPPD design has been awarded through the DOE's STTR (Small business Tech TRansfer) program [21]. Further reading on the LAPPD collaboration can be found later in this report.

\section{MLAP Collaboration}

The Microchannel-Plate-Based Large Area Phototube Collaboration is a Chinese effort developing large spherical phototubes with small, 33mm MCPs as the gain stage. A pair of back-to-back MCP Chevrons share a single anode. Field lines from the photocathode focus photoelectrons on these MCPs. The microchannel plates are used as a gain stage, not for imaging purposes. Thus the MLAPC collaboration hopes to achieve reduced costs by taking MCPs which are adequate for amplification but flawed for use in image tubes. Another interesting aspect of the project is the combination of a transmission-mode and reflection-mode photocathode. Photons incident on the upper hemisphere may produce photoelectrons through the semitransparent cathode on top or they may pass through to the bottom layer, containing a thicker reflection mode photocathode. This is expected to increase the effective single-photon detection efficiency. [22]

\section{Hybrid-APDs}

This is a vacuum tube with enclosed solid state sensor of the avalanche photo-diode (APD). For example, in RICH application, Cherenkov photons enter through the entrance window and generate photoelectrons from a bialkali photocathode. Photoelectrons are accelerated in the electric field of 7-10 $\mathrm{kV}$, and injected into the avalanche photodiode (APD). The APD provides an additional gain of $\sim 40 \mathrm{x}$. The Hamamatsu HAPD for the FRICH Belle-II detector consists of four APD chips, each of which is pixelated into $6 \times 6$ pads for a position measurement, and each pad has a size of $4.9 \mathrm{~mm} \mathrm{x} 4.9 \mathrm{~mm}$. Small HAPD, for example a small Hamamatsu R10647U-01 with bialkali photocathode, have achieved an impressive single photon transit time spread of $\sigma \sim 28 \mathrm{ps}$ when illuminated over $8 \mathrm{~mm}$ dia. area, so they could be considered for some timing applications as well. [23 - 25]

\section{SiPMTs (G-APDs or MMPCs)}

These detectors could be used for RICH detector applications, especially their implementation in the array form. One of their weaknesses, especially for B-factory applications, is their sensitivity to neutron background. Presently, after a does of $\sim 10^{10}$ neutrons $/ \mathrm{cm}^{2}$, these detectors become very noisy. One has to also pay attention to temperature and voltage stability. The present timing resolution limit for a single $3 \mathrm{~mm} \times 3 \mathrm{~mm}$ SiPMT with a $3 \mathrm{~cm}$-long quartz radiator is $\sigma \sim 16 \mathrm{ps} /$ track. One area especially interesting is a development of digital SiPMTs, for example by Philips Company, where each SiPMT micro-pixel will carry information about timing and pulse height. This will lead to superbly percise detectors, both in position and timing. [26 -28]

\section{MRPCs (multi-gap RPCs)}

For very large TOF systems one may consider MRPC technology, as pioneered by the ALICE detector. Its TOF resolution is close to $\sigma \sim 50 \mathrm{ps} /$ track, which is a great achievement, considering that the 
detector's total area ic close to $\sim 150 \mathrm{~m}^{2}$. It provides a good PID performance below $\sim 1 \mathrm{GeV} / \mathrm{c}$. With more gaps in the MRPC multi-electrode structure, one could push the timing resolution towards $\sigma \sim 10$ $20 \mathrm{ps} /$ track. Similarly the STAR experiment reached a timing resolution of better than $100 \mathrm{ps} /$ track. Its total area was $\sim 50 \mathrm{~m}^{2}$. [29-31]

\section{Ring Imaging and DIRC systems}

The first focusing DIRC (FDIRC) was developed for the SuperB collider, where the photon camera would be outside of magnet. Its new focusing optics will make the photon camera $\sim 25 \mathrm{x}$ smaller than the BaBar DIRC, while it timing resolution with H-8500 MaPMTs will be $\sim 10 \mathrm{x}$ better. The photon camera is outside of the magnetic field, which simplifies the detector requirements. The concept relies on a pixel-based reconstruction of the Chrenkov angle; timing is used only to reduce the background, reduce some ambiguities and do chromatic corrections. It would have a similar performance as the BaBar DIRC, i.e., a $3 \sigma \pi / \mathrm{K}$ separation at $\sim 4 \mathrm{GeV} / \mathrm{c}$. One should note that the present FDIRC camera needs 48 H-8500 PaPMTs, however, it would need only 4 LAPPD 8"x8" detectors. Since the SuperB was cancelled, this detector is now one option for the future experiment at Electron-Ion collider at either BNL or JLAB, if one wants to re-use BaBar quartz radiator bar boxes.

One should also mention a TOP counter development for Belle-II, which relies heavily on the high resolution timing at a level of 50-100ps to make the Cherenkov angle reconstruction, and much less on the pixel information. The MCP-PMT detectors have to operate in the magnetic field. This detector will also pioneer a waveform digitizing electronics.

There is a new effort to develop FDIRC detectors for Panda experiment and future experiments at Electron-Ion colliders. These detectors employ various new focusing optics schemes trying improve the Cherenkov angle resolution and achieve a $3 \sigma \pi / \mathrm{K}$ separation up to $\sim 6 \mathrm{GeV} / \mathrm{c}$. This requires to improve both the single photon Cherenkov angle resolution and a total number of measured Cherenkov photons to achieve a resolution of $\sigma_{\text {track }} \sim 1-1.5$ mrads (BaBar DIRC achieved $\sigma_{\text {track }} \sim 2.5$ mrads). For photon detectors they are investigating either the SiPMT-array readout or a new MCP-PMTs with 3-5 micron holes trying to operate up to $\sim 3$ Tesla (for a solution when the photon camera is inside the magnet). There is a vigorous R\&D program both in simulation and hardware areas. This is certainly a new domain in DIRC-like detector development, if successful.

However, for very high momenta PID applications one has to use a gaseous radiator. An example is the forward RICH R\&D development for the Electron-Ion collider. It uses a CsI photocathode with multiGEM photon detector readout.

Another class of RICH detectors worth of mentioning is based on multi-layer Aerogel radiator, coupled to, for example MCP-PMTs or HAPDs. The refraction indices of different Aerogel layers are chosen so one achieves a focusing of the Cherenkov image. The Belle-II plans to use HAPDs, SuperB was considering MCP-PMTs. SuperB detector could achieve a $3 \sigma \pi / \mathrm{K}$ separation at $\sim 5 \mathrm{GeV} / \mathrm{c}$, which is better that FDIRC barrel performance, which aimed for $3 \sigma \pi / \mathrm{K}$ separation at $\sim 4 \mathrm{GeV} / \mathrm{c}$. The SuperB 
forward RICH detector proposal assumed 550 Planacon MCP-PMTs, but it would take only twelve 8"x8" LAPPD MCP-PMTs, if they would be available. [32-36]

\section{Simple TOF systems}

The most simple TOF concept, as it as it avoids complicated 3D data analysis and minimizes chromatic effects, is based on MCP-PMT detector coupled to polished and side-coated fused silica radiator cubes, which are optically isolated from each other. It requires a large number of MCP-PMT detectors, which increases the cost prohibitively at present. Although the prototype beam test reached $\sigma \sim 14 \mathrm{ps} / \mathrm{track}$, the cost was the reason why SuperB did not choose this type of PID concept. However, if MCP-PMT detectors would become cheap in future, one could revive this concept again because it is simple. For example, it may become possible with LAPPD MCP-PMT detectors [3], [37 - 41].

\section{DIRC-like TOF systems}

The early TOF detectors measured time only and typically used scintillator as a radiator. The recent and future TOF detectors measure not only time but $\mathrm{x} \& \mathrm{y}$ coordinates as well, and use a precise quartz radiator, so that a trajectory of each photon can be calculated precisely. The time is constrained by the Cherenkov light information. They are typically called DIRC-like TOF detectors. Examples are (a) TORCH DIRC detector at LHCb, (b) Panda Disc DIRC detector, or (c) FTOF DIRC-like detector for SuperB, etc. The TORCH detector is expected to achieve $\sigma \sim 15 \mathrm{ps} /$ track, Panda Disc detector is expected to reach $\sigma \sim 50-100 \mathrm{ps} /$ track, and FTOF DIRC-like detector for SuperB was supposed to reach $\sigma \sim 70 \mathrm{ps} /$ track. [42 - 44]

\section{MCP-PMT and SiPMT rate capability and lifetime for B-factories}

As an example, the expected rate of H-8500 MaPMT in FDIRC at SuperB would be about 2-3x10 counts $/ \mathrm{cm}^{2} / \mathrm{sec}$ and it would expected to reach $\sim 0.2 \mathrm{C} / \mathrm{cm}^{2} / 10$ years of operation after integrated luminosity of $\sim 50 \mathrm{ab}^{-1}$. Belle-II TOP counter detector, because one deals with smaller detector area, will reach $\sim 0.8 \mathrm{C} / \mathrm{cm}^{2} / 10$ years of operation. The bench tests show that most MCP-PMTs show a stable single photon operation up to $200-300 \mathrm{kHz} / \mathrm{cm}^{2}$ at gain of $10^{6}$. Some MCP-PMTs, for example Hamamastu R10754-00-L4 with 10 micron holes, can reach $\sim 5 \mathrm{MHz} / \mathrm{cm}^{2}$. This means that in both above examples, detectors will be able to handle the rate. However, a possible problem is with the photocathode quantum efficiency (QE). In one case, one expects that the H-8500 MaPMT has a normal PMT aging behavior, and would be fine with the SuperB. However, in the second case, according to Belle-II studies, the best MCP-PMTs can safely reach $0.8-1 \mathrm{C} / \mathrm{cm}^{2}$ before the QE start showing some degree of deterioration, and this is somewhat close to expected a total dose after integrated luminosity of $\sim 50 \mathrm{ab}^{-1}$. More studies are needed. [45]

\section{Bibliography}

[1] K. Scholberg, Supernova Neutrino Detection, Ann Rev of Nuc and Partl Sci, Vol. 62: 81-103 (2012), arXiv:1205.6003 [astro-ph.IM] 
[2] L. Zhan, Y. Wang, J. Cao, L. Wen, Experimental Requirements to Determine the Neutrino Mass Hierarchy Using Reactor Neutrinos, Phys.Rev. D79 (2009) 073007, arXiv:0901.2976 [hep-ex]

[3] H. Frisch, A Differential Time-of-Flight Technique for Collider Detectors, A White Paper.

[4] M. C. Sanchez and M. Wetstein, Using Large Area Micro-channel Plate Photodetectors in the Next Generation Water Cherenkov Neutrino Detectors. Nuclear Physics B Proceedings Supplements, Volume 229, p. 525-525 (2010). Z. Djurcic M. C. Sanchez, M. Wetstein et al., Whitepaper on using Large-Area Picosecond Photosensors for Neutrino Experiments.

[5] B. Ratcliff, The B Factory Detector for PEP-II: A Status Report, SLAC-PUB-5946, 1992.

[6] I. Adams et al., NIM A 583 (2007) 281.

[7] J. Benitez et al., Status of the Fast Focusing DIRC (fDIRC), NIM A 595 (2008) 104.

[8] K. Nishimura et al., NIM A 701 (2013) 115.

[9] R. Abbasi et al, IceCube Collaboration, Calibration and Characterization of the IceCube Photomultiplier Tubes, NIM A vol 618 issues 1-3 (2010) pp 139-152, arXiv:1002.2442 [astroph.IM]

[10] V. Alvarez et al, NEXT collaboration, Radiopurity control in the NEXT-100 double beta decay experiment: procedures and initial measurements, 2013 JINST 8 T01002, arXiv:1211.3961 [physics.ins-det]

[11] K. Nakamura, Neutron damage of SiPMTs, JPS meeting, 2008.

[12] A. Lehmann et al., Systematic studies of MCP-PMTs, NIM A 639 (2011) 144.

[13] Large Arrays of Air Cherenkov Detectors, A White Paper.

[14] The LBNE collaboration, The Long Baseline Neutrino Experiment (LBNE) Water Cherenkov Detector (WCD) Conceptual Design Report (CDR), http://lbne.fnal.gov/reviews/CD1-CDR.shtml arXiv:1204.2295 [physics.ins-det]. J. Brack et. al., Characterization of the Hamamatsu R11780 12 inch Photomultiplier Tube. NIM A (2013), pp. 162-173. 
[15] M. Wurm, et al., The next-generation liquid-scintillator neutrino observatory LENA, arXiv:1104.5620 [astro-ph.IM].

[16] K. Abe, et al., Letter of Intent: The Hyper-Kamiokande Experiment --- Detector Design and Physics Potential, arXiv:1109.3262 [hep-ex].

[17] The IceCube Collaboration, Astroparticle Physics 35 (2012) 615.

[18] C.J. Jillings, R.J. Ford, A.L. Hallin, P.J. Harvey, R.W. MacLeod, H.B. Mak, P. Skensved, and R.L. Stevenson, The Photomultiplier Tube Testing Facility for the Sudbury Neutrino Observatory, NIM A373 (1996) pp. 421-429

[19] MiniBoone B. T. Fleming, L. Bugel, E. Hawker, V. Sandberg, S. Koutsoliotas, S. McKenney and D. Smith, IEEE Trans. Nucl. Sci. 49, 984 (2002); S. J. Brice et al. [MiniBooNE Collab- oration], Nucl. Instrum. Meth. A 562, 97 (2006) [arXiv:1005.3525 [physics.ins-det]].

[20] N. Buchanan, PMT Light Collection Enhancement for LBNE, talk at TIPP 2011

https://indico.cern.ch/contributionDisplay.py? contribId=143\&sessionId=24\& confId=102998

[21] The LAPPD Collaboration, Large Area Picosecond Photodectors, A White Paper.

[22] S. Qian,

http://ssrc.inp.nsk.su/conf/AFAD2013/presentations/WG3/SenQian--V2.1.pdf

[23] I. Adachi et al., Study of a 144 channel multi-anode Hybrid APD for the Belle-II RICH counter, NIM A 639 (2011) 103.

[24] R. Young, Operating the HAPDs in the LHCb RICH counters, NIM A 639 (2011) 94.

[25] T. Iijima, Status and perspectives of vacuum-based photon detectors, NIM A 639 (2011) 137.

[26] S. Korpar, Status and perspectives of solid state photon detectors, NIM A 639 (2011) 88

[27] R. Pestotnik et al., Module of SiPMTs as a detector of individual Cherenkov photons, NIM A 639 (2011) 99.

[28] Ch. Joram, Silicon Photomultipliers Principle, Performance and Some Applications, talk at Bonn University, Germany, December 2012.

[29] C. Williams, TOF with MRPC, Proceedings of the Erice Workshop, 2003. 
[30] Ming Shao et al., STAR MRPC, NIM A 558 (2006) 419.

[31] Y.J. Sun et al., New Prototype MRPC with Long Strips, arXiv:0805.2459.

[32] J. Va'vra et al., Progress on development of the new FDIRC PID detector, NIM A 718 (2013) 541.

[33] K. Nishimura, The time-of-flight counter for Belle II, NIM A 639 (2011) 177.

[34] C. Schwartz et al., Particle identification for the PANDA detector, NIM A 639 (2011) 169.

[35] SuperB, Technical Design Report: INFN-13-01/PI, LAL 13-01, SLAC-R-1003, 2013.

[36] Belle-II Technical Design Report: KEK Report 2010-1, October 2010.

[37] K. Inami et al, NIM A 560 (2006) 303.

[38] J. Va'vra et al., Beam test of a Time-of-Flight detector prototype, NIM A 606 (2009) 404.

[39] A. Ronzhin et al., Development of a 10 ps level time of flight system in the Fermilab Test Beam Facility, NIM A 623 (2010) 931.

[40] J. Va'vra et al., PID techniques: Alternatives to RICH methods, NIM A 639 (2011) 193.

[41] D. Breton et al., High resolution photon timing with MCP-PMTs: A comparison of a commercial constant fraction discriminator (CFD) with the ASIC-based waveform digitizers TARGET and WaveCatcher, NIM A 629 (2011) 123.

[42] M Charles and R. Forty, TORCH: Time of flight identification Cherenkov radiation, Nucl. Inst. \& Meth. A 639 (2011) 173.

[43] E.N. Cowie et al., “A focusing disc DIRC for Panda,” Nucl. Inst. \& Meth. A 639 (2011) 181.

[44] L. Burmistrov et al., "Test of the DIRC-likeTOFprototype for the SuperB experiment," Nucl. Inst. Meth. A 695 (2012) 83; and "FTOF counter in the forward direction," SuperB, Technical Design Report: INFN-13-01/PI, LAL 13-01, SLAC-R-1003, 2013.

[45] A. Lehmann et al., "Systematic studies of MCP-PMTs,” Nucl. Inst. \& Meth. A 639 (2011) 144. 


\section{Solid State Photodetectors}

\section{Contribution of Roger Rusack (University of Minnesota)}

The conversion of optical signals to electronic signals has been a central feature of high energy physics experimental techniques since the very early days. Initially photomultiplier tubes were the principal method used for this purpose. With the advent of the semiconductor industry silicon photodiodes, CCDs and Avalanche Photodiodes (APDs) have become common tools in HEP experiments. Very low-noise CCDs have been used in astronomy and HEP groups have contributed significantly to their application into the infra-red to enable the detection of the high$z$ galaxies. In HEP experiments the challenge of reading out large numbers of channels at a low cost has been met with solid state photodetectors. The introduction of new photodetector techniques to HEP has tracked progress in industry, in most case preceding common adoption by industry.

In the past few years there has been a revolution in our methods of light detection with the introduction of pixelated avalanche photodiodes operating in the Geiger mode with passive or active quenching circuits. These silicon devices are most commonly known as SIPMs or MPPCs. When a photon strikes a SiPM the pixel goes into Geiger mode, saturates and generates a large current. The sum of the currents from all the struck pixels is added to produce a signal which is linear to the flux of photons impinging on the device. After an interval determined by the device design the pixel is brought back to its original state and is fully efficient. As these devices are made using standard CMOS silicon processing techniques they are relatively cheap to manufacture and have been adopted in many applications both inside and outside HEP. One notable property is that their excess noise factor is small $(\sim 1.3)$, comparable to the best PMTs. This is unlike APDs operating in linear mode where the noise increases linearly with the device gain.

The great advantage of these SiPMs is that they are silicon based, behind which lies a large industry that produces material of extraordinarily high quality and with fabrication facilities found around the world. The disadvantage for some applications in HEP is that they are made with silicon. Thus in some applications where the radiation levels are very high the dark current produced by lattice damage can introduce high levels of background into the detector. To mitigate this it is possible to cool the devices, where one can achieve a factor of two reduction in dark count for every interval of $7^{\circ} \mathrm{C}$ that the device is cooled. However for some real-world applications this can be too difficult a drawback. Another drawback to these devices is that silicon has a very short attenuation length for light in the near UV. This makes their use for the detection of scintillation light from noble liquids difficult (for example Xe or Ar for the detection of dark matter). To circumvent this, wavelength shifters are used, with the concomitant loss in photon detection efficiency. 
Because of their relatively low cost, silicon avalanche photodetectors have been used extensively in the field. The CMS lead tungstate calorimeter is readout with a total of 120,000 linear mode APDs and the whole of the NOvA detector is to be readout with APDs. Some of the applications of SiPMs, actual or planned, are the T2K fine grained detector, the upgraded CMS hadronic calorimeter. Outside of the HEP their use in PET detectors is becoming standard.

For future detectors that are being considered by the field they are certain to have many useful applications, especially where a low cost readout of a large number of channels is required. Nevertheless, the limitations of silicon based devices leads to consideration of alternative materials for photodetectors. These can be considered in terms of wavelength of the light to be detected. Short wavelength detection of light is a central problem in the detection of Čerenkov radiation and silicon is not well adapted to this. The detection of UV light, especially for wavelengths less than $360 \mathrm{~nm}$, is particularly difficult for silicon based devices. This is an area of active research in industry for use in monitoring jet engines and for well logging and there is an active area of research in (4H:SiC) Silicon Carbide, which has a bandgap of $3.23 \mathrm{eV}$.

In industry one of the main interests is the detection of light in the near infrared, specifically at wavelengths of $1300 \mathrm{~nm}$ and $1550 \mathrm{~nm}$ for the detection of modulated light in data communications. Silicon photodetectors are not sensitive in this region due to their bandgap of $1.12 \mathrm{eV}$, corresponding to a wavelength cut off of $1100 \mathrm{~nm}$. Interesting detectors where Germanium, which has a bandgap of $0.66 \mathrm{eV}$, are being investigated. The Ge is grown on a silicon substrate, which maintains the advantages of integrated electronics of silicon, combined with the infra-red detection capabilities of germanium.

In another recent development multi-pixel photo-counters based on GaAs and InGaAs, have been reported. These devices should be intrinsically more radiation hard than silicon and may find a place for their application in calorimeters where the radiation levels are higher than can be sustained by silicon devices.

Specific properties that are likely to be relevant for semiconductor photodetectors, apart from the generic low cost, by application are as follows.

Ultra high-energy cosmic ray detection of Čerenkov radiation:

UV sensitivity, fast signal response.

Calorimeters:

HL-LHC: radiation tolerance, large gain band-width product, large dynamic range

ILC: very low cost, large gain-bandwidth product.

High redshift galaxy detection:

Extended sensitivity into the infra-red. 


\section{Crystal Detectors}

\section{Contribution by Ren-Yuan Zhu (California Institute of Technology)}

Crystal detectors have been used widely in high energy and nuclear physics experiments, medical instruments, and homeland security applications. Novel crystal detectors are continuously being discovered and developed in the academy and in industry. In high energy and nuclear physics experiments, total absorption electromagnetic calorimeters (ECAL) made of inorganic crystals have been known for decades for their superb energy resolution and detection efficiency for photon and electron measurements. Crystal ECAL is the choice for those experiments where precision measurements of photons and electrons are crucial for their physics missions. Examples are the Crystal Ball NaI(Tl) ECAL, the L3 BGO ECAL and the BaBar CsI(Tl) ECAL in lepton colliders, the kTeV CsI ECAL and the CMS PWO ECAL in hadron colliders and the Fermi CsI(Tl) ECAL in space. For future HEP experiments at the energy and intensity frontiers, however, the crystal detectors used in the above mentioned ECALs are either not bright and fast enough, or not radiation hard enough. Crystal detectors have also been proposed to build a Homogeneous Hadron Calorimeter (HHCAL) to achieve unprecedented jet mass resolution by duel readout of both Cherenkov and scintillation light, where the cost of crystal detectors is a crucial issue because of the huge crystal volume required. This section discusses several R\&D directions for the next generation of crystal detectors for future HEP experiments.

\section{Crystal detectors with excellent radiation hardness:}

With $10^{35} \mathrm{~cm}^{-2} \mathrm{~s}^{-1}$ luminosity and 3,000 $\mathrm{fb}^{-1}$ integrated luminosity the HL-LHC presents the most severe radiation environment, where up to $10,000 \mathrm{rad} / \mathrm{h}$ and $10^{15}$ hadrons $/ \mathrm{cm}^{2}$ are expected by the CMS forward calorimeter located at $\eta=3$. Superb radiation hardness is required for any detector to be operationally stable in the HL-LHC environment. Fast timing is also required to reduce the pile-up effect. Cerium doped lutetium oxyorthosilicate $\left(\mathrm{Lu}_{2} \mathrm{SiO}_{5}\right.$ or $\left.\mathrm{LSO}\right)$ and lutetium-yttrium oxyorthosilicate $\left(\mathrm{Lu}_{2(1-\mathrm{x})} \mathrm{Y}_{2 \mathrm{x}} \mathrm{SiO}_{5}\right.$ or LYSO) are widely used in the medical industry and have attracted much interest in the HEP community. With high density $\left(7.1 \mathrm{~g} / \mathrm{cm}^{3}\right)$, fast decay time $(40 \mathrm{~ns})$ and superb radiation hardness against gamma-rays and charged hadrons LSO/LYSO crystals are good crystal detectors for future HEP experiments at both the energy and intensity frontiers. LSO/LYSO crystals have been chosen by the Mu2e experiment. A LSO/LYSO crystal based sampling ECAL has also been proposed for the CMS forward calorimeter upgrade for the HL-LHC. Because of its high density, short light path (as compared to the total absorption ECAL) and excellent radiation hardness a LSO/LYSO crystal based sampling ECAL is expected to provide a compact and stable calorimeter at the HL-LHC.

$R \& D$ issues for crystal detectors with excellent radiation hardness include the following aspects: 
- While the gamma-ray and proton induced radiation damage in crystal detectors has been investigated, only limited data are available for neutron induced damage in scintillation crystals. An R\&D program to investigate radiation damage effects induced by gamma-rays, charged hadrons as well as neutrons, in various crystal detectors is needed to address this issue.

- LSO/LYSO crystals are relatively expensive because of the high cost of the raw material, lutetium oxide, and its high melting point. Other fast scintillators (See Table 1 below) as well as fast scintillating ceramics may also be considered. Development of fast and radiation hard crystal and ceramic detectors is of general interest.

- For an LSO/LYSO based Shashlik ECAL, radiation hard photo-detectors as well as wavelength shifters need also to be developed.

\section{Crystal detectors with fast response time:}

Future HEP experiments at the energy frontier, such as HL-LHC, and the intensity frontier, such as the proposed project $\mathrm{X}$, require very fast crystals to cope with the unprecedented event rate.

Table 1 Basic Properties of Fast Crystal Scintillators

\begin{tabular}{|c|c|c|c|c|c|c|c|c|c|c|}
\hline & LSO/LYSO & GSO & YSO & CsI & $\mathrm{BaF}_{2}$ & $\mathrm{CeF}_{3}$ & $\mathrm{CeBr}_{3}{ }^{1}$ & $\mathrm{LaCl}_{3}$ & $\mathrm{LaBr}_{3}$ & $\begin{array}{c}\text { Plastic } \\
\text { scintillator } \\
\text { (BC 404) } 2\end{array}$ \\
\hline Density $\left(\mathrm{g} / \mathrm{cm}^{3}\right)$ & 7.40 & 6.71 & 4.44 & 4.51 & 4.89 & 6.16 & 5.23 & 3.86 & 5.29 & 1.03 \\
\hline Melting point $\left({ }^{\circ} \mathrm{C}\right)$ & 2050 & 1950 & 1980 & 621 & 1280 & 1460 & 722 & 858 & 783 & $70^{\#}$ \\
\hline Radiation Length $(\mathrm{cm})$ & 1.14 & 1.38 & 3.11 & 1.86 & 2.03 & 1.70 & 1.96 & 2.81 & 1.88 & 42.54 \\
\hline Molière Radius (cm) & 2.07 & 2.23 & 2.93 & 3.57 & 3.10 & 2.41 & 2.97 & 3.71 & 2.85 & 9.59 \\
\hline Interaction Length $(\mathrm{cm})$ & 20.9 & 22.2 & 27.9 & 39.3 & 30.7 & 23.2 & 31.5 & 37.6 & 30.4 & 78.8 \\
\hline Z value & 64.8 & 57.9 & 33.3 & 54.0 & 51.6 & 50.8 & 45.6 & 47.3 & 45.6 & - \\
\hline $\mathrm{dE} / \mathrm{dX}(\mathrm{MeV} / \mathrm{cm})$ & 9.55 & 8.88 & 6.56 & 5.56 & 6.52 & 8.42 & 6.65 & 5.27 & 6.90 & 2.02 \\
\hline Emission Peak $^{\mathrm{a}}(\mathrm{nm})$ & 420 & 430 & 420 & $\begin{array}{l}420 \\
310\end{array}$ & $\begin{array}{l}300 \\
220\end{array}$ & $\begin{array}{l}340 \\
300\end{array}$ & 371 & 335 & 356 & 408 \\
\hline Refractive Index ${ }^{b}$ & 1.82 & 1.85 & 1.80 & 1.95 & 1.50 & 1.62 & 1.9 & 1.9 & 1.9 & 1.58 \\
\hline Relative Light Yield ${ }^{a}, c$ & 100 & 45 & 76 & $\begin{array}{l}4.2 \\
1.3\end{array}$ & $\begin{array}{l}42 \\
4.8\end{array}$ & 8.6 & 141 & $\begin{array}{l}15 \\
49\end{array}$ & 153 & 35 \\
\hline Decay Time ${ }^{a}$ (ns) & 40 & 73 & 60 & $\begin{array}{c}30 \\
6\end{array}$ & $\begin{array}{c}650 \\
0.9\end{array}$ & 30 & 17 & $\begin{array}{c}570 \\
24\end{array}$ & 20 & 1.8 \\
\hline $\mathrm{d}(\mathrm{LY}) / \mathrm{dT} \mathrm{T}^{\mathrm{a}, \mathrm{d}}\left(\% /{ }^{\circ} \mathrm{C}\right)$ & -0.2 & -0.4 & -0.1 & -1.4 & $\begin{array}{c}-1.9 \\
0.1\end{array}$ & $\sim 0$ & -0.1 & 0.1 & 0.2 & $\sim 0$ \\
\hline $\begin{array}{l}\text { a. Top line: slow comp } \\
\text { b. At the wavelength o } \\
\text { c. Relative light yield nc } \\
\text { d. At room temperature } \\
\text { \#. Softening point }\end{array}$ & $\begin{array}{l}\text { ent, bottom } \\
\text { he emission } \\
\text { nalized to th } \\
\left.20^{\circ} \mathrm{C}\right)\end{array}$ & $\begin{array}{l}\text { e: fast } \\
\text { ximum } \\
\text { ght yiel }\end{array}$ & $\begin{array}{l}\text { ponent. } \\
\text { f LSO }\end{array}$ & \multicolumn{7}{|c|}{$\begin{array}{l}\text { 1. W. Drozdowski et al. IEEE TRANS. NUCL. SCI, VOL.55, NO.3 (2008) 1391-1396 } \\
\text { Chenliang Li et al, Solid State Commun, Volume 144, Issues 5-6 (2007),220-224 } \\
\text { http://scintillator.Ibl.gov/ } \\
\text { 2. http://www.detectors.saint-gobain.com/Plastic-Scintillator.aspx } \\
\text { http://pdg.Ibl.gov/2008/AtomicNuclearProperties/HTML_PAGES/216.html }\end{array}$} \\
\hline
\end{tabular}

Table 1 above summarizes basic properties of fast crystal scintillators, including oxides (LSO/LYSO, GSO and YSO), halides $\left(\mathrm{BaF}_{2}, \mathrm{CsI}\right.$ and $\left.\mathrm{CeF}_{3}\right)$ and recently discovered bright and fast 
halides ( $\mathrm{CeBr}_{3}, \mathrm{LaCl}_{3}$ and $\left.\mathrm{LaBr}_{3}\right)$, and compares them to typical plastic scintillator (last column). All fast halides discovered recently are highly hygroscopic so that their application needs extra engineering work. Among the non-hygroscopic crystal scintillators listed in Table $1, \mathrm{BaF}_{2}$ crystals are distinguished with its sub-nanosecond fast decay component which produce high scintillation photon yield in the $1^{\text {st }}$ nano-second, just less than highly hygroscopic $\mathrm{CeBr}_{3}$ and $\mathrm{LaBr}_{3}$. This fast component provides a solid foundation for an extremely fast ECAL made of $\mathrm{BaF}_{2}$ crystals. In addition to the good energy resolution and fast response time the photon direction measurements are also important for neutral pion identification through its two photon decays. Most crystal ECAL built so far has no longitudinal segmentation because of the technical difficulty of imbedding readout device in a total absorption calorimeter. One exception is the Fermi ECAL which is a CsI(Tl) strip based hodoscope. With compact readout devices developed in the last decade this issue is less relevant now. A well designed longitudinally segmented ECAL with fast crystal detectors may provide excellent energy resolution, fast timing and fine photon angular resolution, so would serve well for future HEP experiments in general and those at the intensity frontier in particular.

R\&D issues for crystal detectors with very fast response time include the following aspects:

- The slow scintillation component in $\mathrm{BaF}_{2}$ needs to be suppressed by selective doping and/or selective readout by using solar blind photo-detector. The radiation harness of $\mathrm{BaF}_{2}$ crystals needs to be further improved.

- Development of novel fast crystal detectors with sub-nanosecond decay time, e.g. $\mathrm{CuI}$ etc., is of general interest.

- For a longitudinally segmented crystal calorimeter with photon pointing resolution, R\&D on detector design and compact readout devices are required.

\section{Crystal detectors for excellent jet mass reconstruction:}

The next generation of collider detectors will emphasize precision for all sub-detector systems. One of the benchmarks is to distinguish $\mathrm{W}$ and $\mathrm{Z}$ bosons in their hadronic decay mode. Excellent jet mass resolution is required for future lepton colliders at the energy frontier. Following successful experiences with crystal ECAL, a novel HHCAL detector concept was proposed to achieve unprecedented jet mass resolution by duel readout of both Cherenkov and scintillation light. The HHCAL detector concept includes both electromagnetic and hadronic parts, with separate readout for the Cherenkov and scintillation light and using their correlation to obtain superior hadronic energy resolution. It is a total absorption device, so its energy resolution is not limited by sampling fluctuations. It also has no structural boundary between the ECAL and HCAL, so it does not suffer from the effect of dead material in the middle of hadronic showers. With the dual-readout approach large fluctuations in the determination of the electromagnetic fraction of

a hadronic shower can be reduced. The missing nuclear binding energy can then be corrected 
shower by shower, resulting in a good energy resolution for hadronic jets. Such a calorimeter would thus provide excellent energy resolution for photons, electrons and hadronic jets.

$R \& D$ issues for crystal detectors for the HHCAL detector concept include the following aspects:

- Development of cost-effective crystal detectors is crucial because of the unprecedented volume $\left(70\right.$ to $100 \mathrm{~m}^{3}$ ) foreseen for an HHCAL. The material of choice must be dense, cost-effective, UV transparent (for effective collection of the Cherenkov light) and allow for a clear discrimination between the Cherenkov and scintillation light. The preferred scintillation light thus is at a longer wavelength, and not necessarily bright or fast.

- The HHCAL requires R\&D on detector design and on compact readout devices.

Table 2. Basic properties of candidate crystal detectors for HHCAL

\begin{tabular}{|c|c|c|c|c|c|}
\hline Parameters & $\begin{array}{c}\mathrm{Bi}_{4} \mathrm{Ge}_{3} \mathrm{O}_{12} \\
(\mathrm{BGO})\end{array}$ & $\begin{array}{l}\mathrm{PbWO}_{4} \\
\text { (PWO) }\end{array}$ & $\mathrm{PbF}_{2}$ & PbClF & $\begin{array}{c}\mathrm{Bi}_{4} \mathrm{Si}_{3} \mathrm{O}_{12} \\
(\mathrm{BSO})\end{array}$ \\
\hline$\rho\left(\mathrm{g} / \mathrm{cm}^{3}\right)$ & 7.13 & 8.29 & 7.77 & 7.11 & 6.8 \\
\hline$\lambda_{1}(\mathrm{~cm})$ & 22.8 & 20.7 & 21.0 & 24.3 & 23.1 \\
\hline $\mathrm{n} @ \lambda_{\max }$ & 2.15 & 2.20 & 1.82 & 2.15 & 2.06 \\
\hline$\tau_{\text {decay }}(\mathrm{ns})$ & 300 & $30 / 10$ & ? & 30 & 100 \\
\hline$\lambda_{\max }(n m)$ & 480 & $425 / 420$ & ? & 420 & 470 \\
\hline Cut-off $\lambda(\mathrm{nm})$ & 310 & 350 & 250 & 280 & 300 \\
\hline Light Output (\%) & 100 & $1.4 / 0.37$ & $?$ & 17 & 20 \\
\hline Melting point $\left({ }^{\circ} \mathrm{C}\right)$ & 1050 & 1123 & 842 & 608 & 1030 \\
\hline Raw Material Cost (\%) & 100 & 49 & 29 & 29 & 47 \\
\hline
\end{tabular}

Table 2 above compares basic properties of candidate cost-effective crystal detectors for the HHCAL detector concept, such as $\mathrm{PWO}$, scintillating $\mathrm{PbF}_{2}, \mathrm{PbFCl}$ and $\mathrm{BSO}$. Scintillating glasses and ceramics are also attractive for this detector concept.

\section{Summary}

Crystal detectors have been used widely for decades in high energy and nuclear physics experiments, medical instruments and homeland security applications. Future HEP experiments require bright and fast crystal detectors with excellent radiation hardness. Cost-effectiveness is also a crucial issue for crystal detectors to be used in a large volume. Crystal detectors are also an area of fast development. New materials are continuously being found each year. To face these challenges a thorough R\&D program is required to investigate and develop crystal detectors for future HEP experiments in all frontiers. 


\section{The Large Area Picosecond Photodetector (LAPPD) Project}

\section{Henry Frisch and Matt Wetstein on Behalf of the LAPPD Collaboration}

For decades, the high energy physics (HEP) community has relied on photomultiplier tubes (PMTs) to provide low cost, large-area coverage for a wide variety of detector systems. Increasingly, the demands of HEP experiments are pushing for new imaging capabilities, combined with temporal resolutions far better than PMTs can typically offer. A possible replacement for the PMT is the microchannel plate photomultiplier tube (MCP-PMT) [1], a compact detector capable of micron-level spatial imaging and with single photoelectron time resolutions typically measured in tens of picoseconds [refs 2-5].

The Large Area Picosecond Photodetector (LAPPD) Collaboration [6] is developing large-area, flat panel MCP detectors. Central to the LAPPD project is the use of Atomic Layer Deposition (ALD) to mass-produce MCPs from low cost glass substrates. ALD is a batch process whereby materials can be applied uniformly and conformally to large surface areas in bulk, one molecular mono-layer at a time [7]. The structure of LAPPD-made MCPs is provided by borosilicate glass substrates, cut from hexagonally-packed bundles of drawn capillaries with 20-micron pore structures and open-area ratios of $\sim 70 \%$. The substrates are ALD coated, first with a layer of resistive material and then with a secondary-emitting layer. This approach allows independent optimization of the geometric, resistive, and secondary-emission characteristics of MCPs [refs 8-10].

The complete LAPPD MCP detector comprises a photocathode, the MCPs, a segmented anode, and high voltage distribution, integrated within a sealed, flat-panel vacuum envelope. Signals from the MCPs are received by a microstripline anode structure, optimized for high-bandwidth electronics [11]. This delay-line design greatly reduces the necessary channel count, as electronic resources scale only with the square root of the area. Hit positions are determined by the signal centroid in the direction perpendicular to the striplines and, along the strips, by the difference in the arrival time at the two ends of the striplines. The digitization electronics uses low-power CMOS technology [12]. Arrival times and gains of the pulse trains are measured by waveform sampling, which offers pulse shape and charge measurement, multiple hit capability, as well as the best timing resolution [13]. The entire package is hermetically sealed in a flat-panel glass body [14].

Tests of working LAPPD detector systems have demonstrated 8" MCPs with gains above $10^{7}$. Time resolutions approach single picoseconds in the large signal limit ( $\mathrm{N}$ photoelectrons $>100)$ and better than 50 picoseconds for single photoelectrons. The position of incident photons in the direction parallel to the stripline anode is determined by the difference in signal arrival time at the two ends of our delay line. This differential time resolution was measured to be better than $20 \mathrm{psec}$ for single photoelectrons, which would translate to a spatial resolution of roughly $2 \mathrm{~mm}$ in the direction parallel to the strips. Position in the transverse direction is determined by a weighted centroid of the charge collected on each strip and is measured to be better than $1 \mathrm{~mm}$. [15] 
Nominally LAPPDs will be made with conventional photocathodes. The collaboration has been able to demonstrate 8 " x 8" photocathodes with quantum efficiency above 20\% [16]. As these detectors are commercialized, it is safe to assume that they can be made with QE's comparable to standard phototubes available on the market.

Another major milestone of the project was the production of the PSEC4 ASIC, a fast sampling chip capable of digitizing MCP pulses at 15 giga-Samples/second, with an analog bandwidth of better than 1 $\mathrm{GHz}$ and less than $1 \mathrm{mV}$ noise [12]. The ASIC is made using low-power and scalable CMOS technology. Along with the PSEC4, a full front-end FPGA-based DAQ system is now being tested. The near-complete detector system is now being tested on a "demountable LAPPD", a working seal-glass tube, whose only differences from the final design goal are that: 1) the tube is actively pumped rather than hermetically sealed; 2) the seal between the top window and the tube body is with an O-ring rather than an indium seal; and 3) the photocathode is a thin Aluminum layer rather than a bialkali, as the demountable is assembled in air. The purpose of the demountable LAPPD setup is to test a complete, end-to-end detector system, including our own LAPPD-designed front-end electronics. The system allows readout of all 60 detector channels, 30 per side. A four anode chain constitutes one row of three in a "Super Module" (SuMo) - a large-area detector system designed to reduce channel counts by sharing the same delay line pattern for several MCPs [13].

The Department of Energy awarded a Phase I STTR grant (Section 38) to Incom Inc for technology transfer for LAPPD detectors [17]. At Berkeley Space Science Laboratory a parallel effort is underway to produce MCPs with an alternative ceramic-body design.

LAPPDs may prove useful in a wide variety of non-HEP contexts, such as medical imaging, neutron detection, and homeland security. The HEP community could greatly benefit from the economies of scale achievable with a larger market.

\section{Bibliography}

\section{[1] J.L. Wiza, Microchannelplate Detectors, Nucl. Instrum. Methods 162 (1979) 587-601.}

[2] J. Milnes and J. Howorth, Picosecond Time Response of Microchannel Plate PMT Detectors, Proc. SPIE 5580 (2005) 730-740.

[3] M. Akatsu, Y. Enari, K. Hayasaka, T. Hokuue, T. Iijima, K. Inami, K. Itoh, Y. Kawakami, et al, MCP-PMT Timing Property for Single Photons, Nucl. Instrum. Methods A528 (2004) 763-775.

[4] K. Inami, N. Kishimoto, Y. Enari, M. Nagamine, T. Ohshima, A 5-ps TOF-Counter With an MCP$P M T$, Nucl.Instrum.Methods A 560 (2006) 303-308. 
[5] A. Tremsin and O. Siegmund, Spatial Distribution of Electron Cloud Footprints From Microchannel Plates: Measurements and modeling, Rev. Sci. Instrum. 70, 3282-3288 (1999).

[6] The Large Are Picosecond Photodetector Collaboration: http://psec.uchicago.edu

[7] S.George, Atomic Layer Deposition: An Overview, Chemical Reviews 110 (2010) 111-131.

[8] A. Mane, Q. Peng, J. W. Elam, D. C. Bennis, C. A. Craven, M. A. Detarando, J. R. Escolas, H. J. Frisch, S. J. Jokela, J. McPhate, M. J. Minot, O. H. W. Siegmund, J. M. Renaud, R. G. Wagner, and M. J. Wetstein, A novel atomic layer deposition method to fabricate economical and robust large area microchannel plates for photodetectors, Phys. Procedia, vol. 37, pp. 722-732, 2012.

[9] N. Sullivan, P. de Rouffignac, D. Beaulieu, A. Tremsin, K. Saadatmand, D. Gorelikov, H. Klotzsch, K. Stenton, S. Bachman, R. Toomey, Novel microchannel plate device fabricated with atomic layer deposition, Proceedings of the Ninth International Conference on Atomic Layer Deposition.

[10] S. Jokela, I. Veryovkin, A. Zinovev, J. Elam, Q. Peng, A. Mane, The Characterization of Secondary Electron Emitters for Use in Large Area Photodetectors, Application of Accelerators in Research and Industry AIP Conf. Proc. 1336 (2011) 208-212.

[11] H. Grabas, R. Obaid, E. Oberla, H. Frisch, J. Genat, R. Northrop, D. McGinnes, B. Adams, and M. Wetstein, RF Stripline-anodes for Psec Large-area, MCP-based Photodetectors, Nucl. Instrum. Methods 711, 124-131 (2013). http://psec.uchicago.edu/library/doclib/documents/211

[12] E. Oberla, H. Grabas, J.-F. Genat, H. Frisch, K. Nishimura, and G. Varner, A $15 \mathrm{GSa} / \mathrm{s}, 1.5 \mathrm{GHz}$ Bandwidth Waveform Digitizing ASIC for Fast Detector Readout, accepted for publication in Nucl. Instrum. Methods Phys. Res. A. http://psec.uchicago.edu/library/doclib/documents/218

[13] B. Adams, M. Chollet, A. Elagin, E. Obaid, E. Oberla, A. Vostrikov, M. Wetstein, P. Webster, A Test Facility for Large-area Microchannel Plate Detctor Assemblies Using a Pulsed sub-Picosecond Laser, Rev. $\quad$ Sci. Instrum. 84, $061301 \quad$ (2013); doi: 10.1063/1.4810018 http://psec.uchicago.edu/library/doclib/documents/214

[14] An Internal ALD-Based High Voltage Divider and Signal Circuit for MCP-based Photodetectors draft in preparation $\mathbf{J}$ Inst.

[15] Timing Characteristics of Large-Area Microchannel Plate Detector with Microstripline Anodes draft in preparation for Nucl. Instru. Methods A. 
[16] O. H. W. Siegmund, Member, IEEE, J. B. McPhate, S. R. Jelinsky, J. V. Vallerga, A. S. Tremsin, Member, IEEE, R. Hemphill, H. J. Frisch, R. G. Wagner, J. Elam, A. Mane, and LAPPD COLlABORATION, Large Area Microchannel Plate Imaging Event Counting Detectors With SubNanosecond Timing, IEEE Transactions on Nuclear Science, Vol. 60, No. 2, April 2013

http://psec.uchicago.edu/library/doclib/documents/225

[17] DOE STTR Phase I, Release I Award to Incom, Inc. for LAPPD Commercialization - Fully Integrated Sealed Detector Devices, http://science.energy.gov/sbir/awards/ 


\title{
Transition Edge Sensors for High Energy Physics
}

\author{
Contribution of C. L. Chang, B. Cabrera, J. E. Carlstrom, S. Golwala, K. D. \\ Irwin, A. T. Lee, M. Pyle
}

Transition Edge Sensors

Transition Edge Sensors (TES) are ultra-sensitive thermometers consisting of a thin superconducting film that is weakly heat-sunk to a bath temperature much lower than the superconductor Tc (see Fig. 1, left). The principles of operation are simple to understand. By supplying electrical power to the TES, we can raise the temperature of the sensor so that the film is in the middle of its superconducting-to-normal transition (see Fig. 1, right). If the electrical power is supplied via a voltage bias, a negative feedback loop is established. Small changes to the TES temperature, arising from thermal fluctuations (noise) or changes in the absorbed power from a source (signal), lead to large changes in the TES resistance. The change in resistance creates a canceling effect because increases (or decreases) in temperature produce decreases (or increases) in Joule heating power. This negative electro-thermal feedback is very strong because the transition is very sharp. It linearizes the detector response and expands the detector bandwidth. The fundamental noise arises from thermal fluctuations. At the sub-K operating temperatures for typical TES devices, these fluctuations are small giving the TES excellent resolving power. An extremely important aspect of TES devices is that they are low-impedance $(<1 \Omega)$ and can be multiplexed with modern-day Superconducting QUantum Interference Device (SQUID) multiplexers. This makes TESbased detectors amenable to large array applications.
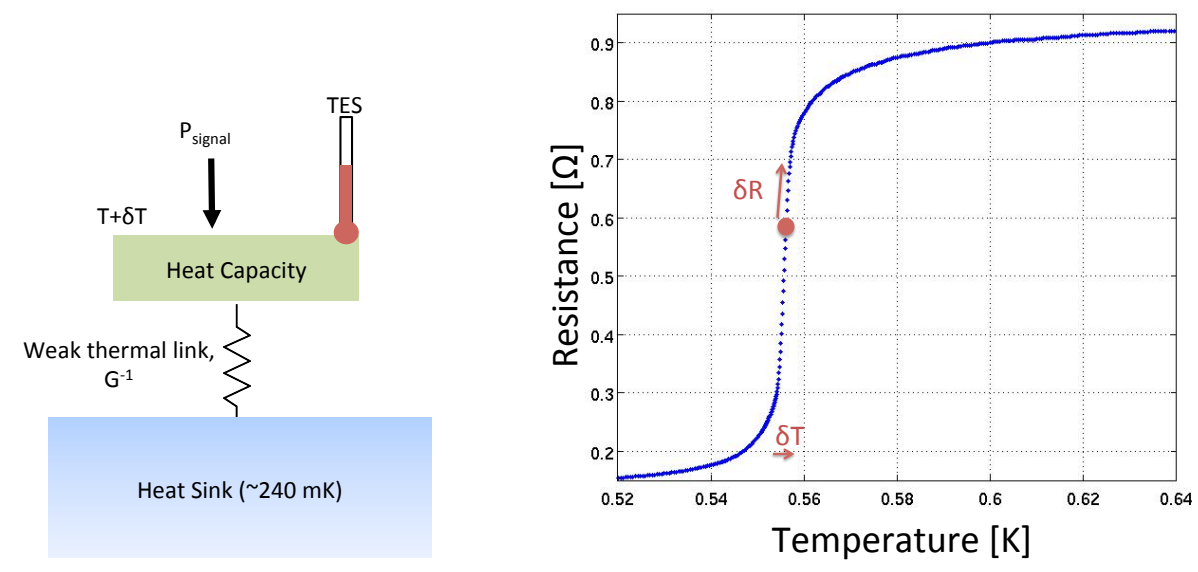

Figure 1: Left: Illustration of a thermal circuit for a typical Transition Edge Sensor (TES) detector highlighting the principles of signal detection. A weakly thermally sunk heat capacity absorbs power, Psignal, which is to be measured. Variations in the absorbed power change the heat capacity's temperature, which is measured by a TES operating under strong electro-thermal feedback. Right: Plot of resistance versus temperature for a typical TES illustrating the principles of negative electro-thermal feedback. The TES is voltage biased into the middle of its superconducting-to-normal transition. Small changes in the TES temperature produce large changes in the TES resistance. Since the TES is voltage biased, an increase (or decrease) in the temperature produces an increase (or decrease) in the resistance leading to a decrease (or 
increase) in the Joule heating power supplied by the bias. This canceling effect corresponds to a strong negative electro-thermal feedback making the current through the TES nearly proportional to Psignal.

\section{TES Detectors and the Cosmic Frontier}

TES detectors were invented within the context of Dark Matter searches. The development of TES detectors with exceptional background rejection has pioneered the field of direct Dark Matter detection. The strong detector history has provided a solid understanding of the fundamental detector physics along with a proven track record of scientific leadership. Over the course of the next decade, TES-based Dark Matter detectors continue to be a promising technology. There is a clear path towards a G3 ton-scale Dark Matter observatory and on-going R\&D focused on reducing the detector energy threshold which would increase the sensitivity of TES Dark Matter detectors to lower mass WIMPs.

Over the last decade, TES bolometers have also had a profound impact on CMB measurement. CMB detector technology is sensitivity limited where the measurement is fundamentally limited by the shot noise of the photons being measured. As such, increases in the sensitivity of experiments is possible only through increasing the size of the focal plane. At the end of the last century, CMB detector technology resembled that of infrared imaging at its inception: limited hybrid focal plane arrays involving a small number of individual detectors. The implementation of the HEP invented TES to CMB bolometers together with modest multiplexing has enabled kilo-pixel focal plane arrays which have ushered in an era of unprecedented CMB surveys. The next decade of CMB measurement and instrumentation development is focused on Stage III ( $>10,000$ detector elements) and Stage IV ( $>100,000$ detector elements) experiments with each stage having an order of magnitude increase in experiment mapping speed. The Stage III and Stage IV sensitivities translate directly into the size of fielded focal planes and TES-based focal planes are the only CMB detector technology with a clear path to Stage IV sensitivities.

\section{Challenges for TES detectors R\&D}

The technology development challenges for the next decade of TES detectors are similar across both applications within the HEP Cosmic Frontier and are broken down as follows:

- Extending the reach of active detector elements For Dark Matter TES detectors, this challenge involves reducing the detector noise by nearly an order of magnitude. Such a reduction would lower the detection threshold by a factor of ten opening up sensitivity to new parameter space for lowmass WIMPs. For CMB bolometers, this challenge involves developing reliable fabrication of highbandwidth ultra-low loss superconducting microstrip which is utilized for coupling the bolometer to mm-wave radiation.

- Large increases in the volume and throughput of detector production Upcoming TES applications for both Dark Matter and CMB experiments require dramatic increases in the fabrication of TES detectors. In order to achieve the required production throughput, access to micro-fabrication resources is required with exclusive control of the thin film deposition systems. Together with developing the fabrication processes and tooling for mass production of TES devices, an extensive program of detector testing, characterization, and quality control is necessary to support the increased production capacity. Additionally, improvements in the multiplexed readout of TES detectors is required for high-bandwidth operation of future TES detector arrays with thousands of elements. 
The US leads the world in the implementation of TES technology to the forefront of HEP science. TES detectors have driven the field of Dark Matter detection and are the best technology for ongoing leadership in CMB experiment. The technical challenges for the next decade of TES development reflect the fact that the superconducting technology critical for future Dark Matter and CMB science is not available through commercial industry. As such, HEP resources and facilities are required to sustain ongoing US leadership in these fields.

\section{Broader Impact}

TES technology is exceptionally versatile with diverse applications beyond those listed above. Over the last decade TES detectors have been implemented in the following:

- measurements of the neutrino mass via both double beta decay and beta decay endpoint measurements

- quantum information and cryptography

- spectrophotometry for optical astronomy

- focal planes for sub-mm astronomy

- detectors for national security include thermal imaging and nuclear non-proliferation

- micro-calorimeter arrays x-ray astrophysics

- $\quad$ spectroscopy at synchrotron light sources

The spectrum of TES applications illustrate how ongoing HEP investment into TES development can produce technologies that are readily exported to programs and disciplines beyond the scope of HEP science. 


\section{Instrumentation challenges for Direct Detection of Dark Matter Contribution of James Fast, Jeter Hall, Eric Hoppe and John Orrell (PNNL)}

In this section we review dark matter sensor technologies exclusive of the liquid noble gas systems. Common to all of these sensors, and the liquid noble gas detectors, is the overarching challenge of ultra-pure materials and assay techniques, covered at the end of this contribution.

\section{HPGe Spectrometers}

Current and planned experiments using high-purity germanium (HPGe) spectrometers include CoGeNT, C4, MAJORANA, and GERDA. While the MAJORANA and GERDA experiments are primarily searches for neutrinoless double-beta decay, if they can achieve the required low energy threshold - even on a fraction of their detector mass - they will be formidable DM detectors. The main challenges for these experiments are background reduction (see materials and assay section) and lowering energy thresholds to $<100 \mathrm{eV}$.

CoGeNT/C4 employs commercially-available PPC (p-type point contact) germanium crystals to drastically reduce the capacitance of the detector element, and with it the contributions from several sources of electronic noise. This results into a present energy threshold of $0.45 \mathrm{keVee}(\sim 2$ keVnr), an order of magnitude improvement over conventional coaxial germanium detectors [cogent1]. The technology is inexpensive and stable enough for a demonstrated continuous operation spanning several years [cogent2], i.e., highly suitable for a search for the annual modulation signature from low-mass WIMPs, testing the DAMA/LIBRA anomaly. For $1 \mathrm{pF}$ capacitances like those obtained in these PPCs, the theoretically reachable electronic noise is however one full order of magnitude lower (around 50 eVee, commonly achieved in state-of-theart x-ray semiconductor detectors). The dominant source of residual noise in past CoGeNT detectors has been identified as parasitic capacitances affecting the first stage of signal amplification. Measures against this source of noise have been implemented in the PNNL-built cryostat for the first $\mathrm{C} 4$ detector [cogent3], about to be tested at the time of this writing. Beyond those, the manufacturer of these PPCs (Canberra Industries) has embarked in studies of further optimization of intra-contact surface passivation and point-contact design, both able to generate

a further noise reduction. A team at the University of Washington works on a new design of pulse-reset preamplifier, again able to improve the noise budget. In addition to these efforts, the University of Chicago group is developing FPGA-based intelligent triggering schemes capable to reduce the effective detector threshold. The goal for future PPC technology is to further reduce the threshold in these devices as close as possible to the 50 eVee $(\sim 0.2 \mathrm{keVnr})$ limit, while increasing the crystal mass by a factor of a few (the first C4 crystal weights in at $1.3 \mathrm{~kg}$, in contrast to the $0.4 \mathrm{~kg}$ CoGeNT PPC presently operating in Soudan). Sensitivity predictions can be found in [cogent3].

\section{Silicon CCDs}

The DAMIC experiment is using fully-depleted thick (320 micron) CCD's developed for astronomical survey instruments (SNAP/JDEM, DES) at LBNL. These sensors have been 
demonstrated in not-so-low background environments at Fermilab and have recently been deployed and are operating at SNOLAB. Initial data collected at SNOLAB indicates that backgrounds have not been reduced compared to shallow underground operation at Fermilab revealing considerable uranium and thorium backgrounds coming from the detector mounting structures. In addition to the common theme of radiometric background reduction, the most significant challenge for this technology will be increasing mass to kg levels. These sensors have extremely low thresholds (few eV) and hence are sensitive to much lower WIMP mass scales than any other technology, and hence are complimentary to all other searches.

\section{Bubble Chambers}

The COUPP/PICASSO experiments utilize bubble chambers to search for WIMP dark matter. Bubble chambers provide a "perfect" threshold cutoff that rejects low ionization loss events coming from electromagnetic backgrounds. COUPP is currently operating a $60 \mathrm{~kg}$ CF3I bubble chamber at SNOLAB.

The primary backgrounds for this technology are surface events coming from alpha-emitting materials in the quartz containment vessel and from neutrons penetrating the shielding. The technology is currently being scaled up to the $\sim 100 \mathrm{~kg}$ level and a $\sim 500 \mathrm{~kg}$ experiment has been proposed. Sensor challenges include vessel radiopurity and fluid purification systems needed to achieve the required background levels.

\section{Solid Xenon}

The solid (crystalline) phase of xenon inherits most of the advantages of using liquid xenon as a detector target material for low energy particles; transparency, self-shielding, absence of intrinsic background, and ionization drift. In the solid phase, even more scintillation light yield $(\sim 60 \mathrm{keV})$ is reported compared to the liquid phase $(\sim 40 \mathrm{keV})$. Operation at sub-Kelvin temperature is natural for the solid phase, using superconducting sensors to read out photon, ionization, and phonon signals.

Most of the properties of solid xenon have been measured in a small volume or in thin film in the early 1970s through 1990s, but there were no systematic studies successfully carried out with large-scale solid xenon. In January 2010 Fermilab grew approximately a kilogram of transparent solid phase of xenon. Ongoing R\&D at Fermilab is focused on scintillation light read out and electron drift in the solid xenon.

There are two remaining challenges for advanced detector development. (1) For solar axion searches, single-crystal xenon is favorable. The University of Enlargen (Germany) is planning to carry out the crystallography study of the solid xenon. (2) For neutrinoless double-beta decay searches, phonon read out from the solid xenon is favorable which requires millikelvin operation with superconducting phonon sensor technology. Characteristics of xenon at millikelvin temperatures have rarely been studied and deserves further R\&D. 


\section{Materials and Assay}

The general approach to radioactive backgrounds is to reduce, shield, and finally discriminate against interactions with known types of ionizing radiation. The menagerie of direct detection experiments is a tribute to the clever approaches that have been developed to discriminate against electronic recoils from gamma and beta backgrounds. Typically, neutron backgrounds are the most insidious because they induce nuclear recoils identical to the expected WIMP signals and self-shielding against neutrons typically cuts a prohibitive amount of the active volume. Many of these material requirements are similar to other rare event searches in these energy ranges, for example low energy neutrino and neutrinoless double-decay experiments. However, the emphasis on neutron backgrounds is different than the emphasis on, say, $2 \mathrm{MeV}$ gamma rays in germanium based neutrinoless double-decay experiments.

\section{Materials}

Since neutrons backgrounds almost always limit an experiment, there is a special emphasis on removing uranium and thorium contamination since they can produce neutrons through spontaneous fission or $(\alpha, n)$ depending on the matrix in which they are embedded. The amount of uranium and thorium contamination allowed in particular materials varies due to the location and matrix, but taking spontaneous fission as an inevitable process, a contamination of $1 \mathrm{mg}$ of ${ }^{238} \mathrm{U}$ results in a production of 1.2 neutrons per day. In context, the next generation dark matter experiments have background goals approaching $<1$ event/year in detectors with fiducial masses of $\sim 1$ ton.

Although there are a variety of dark matter experiments, a convenient dissection of the experiments is that they are made of shielding materials, detector materials, and electronics.

\section{Shielding}

Traditionally, small detectors have used a mixture of lead to shield external gamma rays, and hydrogenated material, such as polyethylene, to shield external neutrons. As dark matter detectors have grown in size, more and more experiments are moving to water based shielding. Large ( $\sim 3$ meter thick) shields are common as these are thick enough to serve as both gamma and neutron shielding.

\section{Detector Materials}

All dark matter detectors consist of some active element, germanium and xenon as examples, which are instrumented and contained in some sort of vessel. Some experiments require pressure vessels and others require vacuum vessels for containment. These vessels must satisfy leak and mechanical constraints. Materials currently used for containment include stainless steel, copper, and titanium. Generally, the radioactivity from these materials must be less than $\sim 10$ $\mu \mathrm{Bq} / \mathrm{kg}$ to meet the specifications of dark matter experiments.

\section{Electronics}

The sensitive volumes are instrumented with various sensors and electronics. Many of the scintillator-based detectors utilize photomultiplier tubes (PMTs) and developing low 
radioactivity light detectors has been an active area of research. However, PMTs are often the dominant source of both neutron and gamma backgrounds in experiments based on the noble elements. Even with their high backgrounds, PMTs offer the low noise, high sensitivity, and large areas required by dark matter experiments, so they are the photodetector used in almost all scintillator based experiments. Other lower background devices such as large area avalanche photodiodes have been successfully deployed in other rare event searches, but the requirement for single photoelectron sensitivity currently precludes their use in dark matter detectors. Silicon photomultipliers are an attractive option, but their small areas and high dark rates are prohibitive.

Other, more basic, electronics components used in almost all dark matter experiments are potential sources of unwanted backgrounds including cables, circuit board substrates, resistors, and capacitors. Devices made from ceramics or with significant glass content typically contain significant uranium, thorium, and potassium contamination. A large amount of effort is spent by each collaboration to screen potential components and substrates to find sufficiently low background pieces. Often the radioactivity requirements are coupled with other unique requirements such as thermal conductivity.

\section{Surface Contaminations}

It is not sufficient to screen materials for bulk contamination of radioactivity. Surface contamination, often from chemical deposition or radon plate out, is an important consideration for many rare event searches. This is especially true for surfaces that face the active detector material. Typical surface contaminants are due to chemical deposition and radon plate out, so these contaminants include isotopes that $\alpha$-decay inducing worries about $(\alpha, n)$ neutron backgrounds from surfaces that do not have line of sight to the sensitive regions of detectors. The accumulation of radon daughters during material storage and handling is an important consideration for dark matter detector construction. These backgrounds can be difficult to assay at levels important for modern dark matter experiments since direct alpha, or low energy beta, screening is the most sensitive to radon daughters such as ${ }^{210} \mathrm{~Pb}$.

\section{Assay}

Assay of materials for radioactive isotopes is required to reduce risk and improve the scientific output of each dark matter experiment. Simulations of specific detector geometries is required to give contamination budgets for specific components. Then each candidate material is assayed to qualify a material or commercial product for a specific detector component. The most common methods of assay are through gamma counting with high purity germanium detectors (HPGe), mass spectroscopy, and neutron activation analysis (NAA). The requirements on sensitivity, sample size, and isotopic analysis will drive the decision on assay technique. High sensitivity HPGe analysis requires large samples, up to a few kilograms of material, whereas mass spectroscopy and NAA can achieve important radioactive contamination levels with $\sim 1 \mathrm{~g}$ samples.

HPGe assay is a useful tool for material assay. The ${ }^{238} \mathrm{U}$ and ${ }^{232} \mathrm{Th}$ decay chains emit characteristic gamma rays, however the decay chains can be broken either through chemical means or at the 
radon break points, so gamma spectroscopy requires care at the stage of interpretation. Pure gamma emitting isotopes such as ${ }^{40} \mathrm{~K}$ are ideal candidates for HPGe assay.

Mass spectroscopy is a useful tool because it will measure exact isotopes of interest and extrapolations through decay chains is not required. Additionally, penetrating gamma rays are not required for elemental identification. Glow discharge mass spectroscopy (GDMS) is a useful tool because a variety of isotopes can be measured at the same time and sample preparation is minimal. However, GDMS has poor sensitivity, so is typically only useful for prescreening materials for more sensitive assay techniques. Inductively coupled plasma mass spectroscopy (ICPMS) is more sensitive to isotopes of interest and is a important tool for modern dark matter experimentalists. The most sensitive ICPMS requires dissolving samples for concentration, and special techniques are required to perform the most sensitive ICPMS on the large variety of materials required for dark matter detectors. ICPMS does suffer from important interferences that reduce the sensitivity for certain isotopes, for example ${ }^{40} \mathrm{~K}$ is difficult due to the argon gas used in ICPMS.

NAA is sensitive to a number of isotopes of interest for the dark matter community. Again this technique can probe specific isotopes of interest, such as ${ }^{238} \mathrm{U}$, without relying on decay chain assumptions. NAA requires careful sample preparation and specialized analysis. NAA, especially when combined with radiochemical separation, can result in some of the best minimum detection levels currently possible.

\section{Custom Materials}

It has been shown that modern metallurgical techniques and special attention to polymerization precursors can reduce the radioactive contamination in materials. Collaborations interested in rare event searches have shown that custom materials can be developed when commercial materials do not meet specifications in terms of radioactivity.

For example, high-Z construction materials can be purified using electrochemical techniques to levels resulting in $<1 \mu \mathrm{Bq} / \mathrm{kg}{ }^{238} \mathrm{U}$ and ${ }^{232} \mathrm{Th}$ activities. Not only can the radioactivity be reduced in these materials, but the mechanical properties can be controlled better than in typical highthroughput industrial techniques. This has been demonstrated for copper by the MAJORANA Collaboration, and could also be accomplished for other important metals such as gold, lead, nickel, tungsten and iridium as well as some metal alloys such as a number of copper alloys.

Custom low background electronics components such as capacitors, resistors, and cabling could be produced in sufficient quantities that the up-front development costs could be shared economically across future experiments reducing the need to constantly assay large numbers of these components.

\section{Summary}

Low background materials are important for dark matter research and their importance will only grow over the next decades. Insufficient care with seemingly insignificant detector components can drastically reduce the scientific impact of experiments [xmass1], however sufficient care can produce experimental backgrounds within desired specifications [exo1]. The ability to source, 
assay, and keep materials free of radioactive contamination is crucial to reducing risk and improving scientific output from the efforts to directly detect WIMP dark matter interactions with terrestrial detectors.

\section{Bibliography}

[cogent1] P.S. Barbeau, J.I. Collar and O. Tench, JCAP 09 (2007) 009; C.E. Aalseth et al., Phys. Rev. Lett. 101 (2008) 251301; Erratum ibid 102 (2009) 109903; Phys. Rev. Lett. 106 (2011) 131301; Phys. Rev. Lett. 107 (2011) 141301.

[cogent2] C.E. Aalseth it et al., arXiv:1208.5737, submitted to Phys. Rev. D.

[cogent3] R.M. Bonicalzi et al., Nucl. Instr. Meth. A712 (2013) 27.

[xmass1] K. Abe et al., Phys. Lett. B719 (2013) 78.

[exo1] M. Auger et al., Phys. Rev. Lett. 109 (2012) 032505. 


\title{
3D Technologies for Large Area Trackers
}

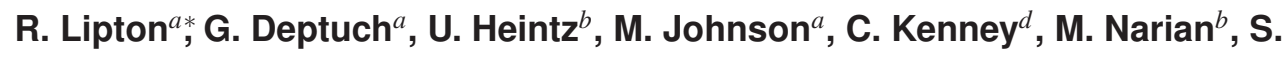

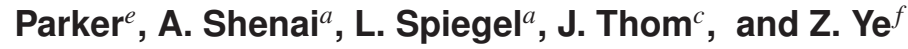 \\ ${ }^{a}$ Fermilab, \\ P.O. Box 500, Batavia, Il , USA \\ ${ }^{b}$ Brown University, \\ Providence, RI, USA \\ ${ }^{c}$ Cornell University, \\ Ithaca, NY, USA \\ ${ }^{d}$ SLAC National Accelerator Laboratory \\ Menlo Park, CA, USA \\ ${ }^{e}$ University of Hawaii, \\ Honolulu, HI, USA \\ ${ }^{f}$ University of Ilinois, Chicago, \\ Chicago, Il, USA \\ E-mail: liptonefnal.gov
}

\begin{abstract}
We describe technologies which can be developed to produce large area, low cost pixelated tracking detectors. These utilize wafer-scale 3D electronics and sensor technologies currently being developed in industry. This can result in fully active sensor/readout chip tiles which can be assembled into large area arrays with good yield and minimal dead area. The ability to connect though the bulk of the device can also provide better electrical performance and lower mass.
\end{abstract}

${ }^{*}$ Correspondingauthor. 


\section{Contents}

$\begin{array}{ll}\text { 1. Introduction } & 1\end{array}$

2. Technologies 2

2.1 3D Circuits 2

2.2 Active Edge Sensors 3

2.3 Oxide Bonding 4

2.4 Interconnect 4

3. Large Area Assembly 5

3.1 Tiles with Active Edge Sensors 5

3.2 Post-Processed Tiles 5

3.3 Die-to-Die tiles 6

$\begin{array}{lll}3.4 & \text { Interposer-based Array } & 6\end{array}$

$\begin{array}{lll}\text { 4. } & \text { Prospects } & 7\end{array}$

\section{Introduction}

In the next generation of collider experiments detectors will be challenged by unprecedented luminosity and data volumes. We will need to build large area arrays (100's of meter ${ }^{2}$ ) of highly pixelated detectors with minimal dead area and at reasonable cost. Current fine pitch bump bonding technologies require individual placement of chips on sensors followed by a solder melt cycle. Technologies which provide wafer-scale interconnect offer prospects of lower cost, finer pitch and lower mass interconnects between sensors and readout chips. In addition 3D interconnect technology allows connections through the bulk of the silicon, providing low inductance and capacitance paths for signal and power connections. Table 1 summarizes current and projected costs and yields of various bonding technologies.

In the past pixel sensor arrays have been limited in the module area by:

1. Space needed at the edges of the detectors to reduce the field near the damaged dicing cut regions. These regions act as charge emitters and can cause unacceptably large currents in the edge strips.

2. Die size of the Readout Integrated Circuit (ROIC), determined by the die yield and reticule area

3. The need to provide edge locations for wire bonds for connection to the ROIC. 
The development of active edge sensors by S. Parker, C. Kenney and collaborators has addressed the first problem [1] [2] [3]. Recent work on the large area FEI4 chip [4] has shown that a large pixel chip can be produced with good yield. Processes related to 3D electronics can solve the third problem. The combination of all three can result in a wafer scale fabrication of tiles or arrays which can be integrated into large modules with high yield and relatively low cost. Using such tiles, large area pixelated modules can be assembled with known good integrated sensor/readout die with large pitch backside bump bond interconnects.

Table 1. Current and projected costs and yields for sensor/readout integration technologies.

\begin{tabular}{|c|c|c|c|}
\hline Component & Current or projected cost & Yield & Comment \\
\hline Readout IC & $\$ 8 / \mathrm{cm}^{2}[5]$ & $65-70 \%[6]$ & $\begin{array}{l}\text { Current 3D wafer cost and } \\
\text { FEI4 prototype yield }\end{array}$ \\
\hline Active Edge Sensors & $\$ 53 / \mathrm{cm}^{2}$ & $\approx 90 \%$ & $\begin{array}{l}\text { Current cost for prototype } \\
150 \mathrm{~mm} \text { wafers }\end{array}$ \\
\hline Silicon Strip Sensors & $\$ 10 / \mathrm{cm}^{2}$ & $\approx 100 \%$ & CMS tracker costs \\
\hline $\begin{array}{c}\text { Bump bonding } \\
\text { (2007) }\end{array}$ & $\$ 213 / \mathrm{cm}^{2}$ & $98 \%[7]$ & $\begin{array}{c}\text { CMS forward pixel costs } \\
\text { Yield } \equiv<20 \text { bad bumps/chip }\end{array}$ \\
\hline $\begin{array}{l}\text { Bump bonding } \\
\text { (2012) }\end{array}$ & $\$ 62 / \mathrm{cm}^{2}$ & - & CMS forward pixel upgrade \\
\hline $\begin{array}{c}\text { Wafer scale } \\
\text { DBI bonding }\end{array}$ & $\$ 0.04 / \mathrm{cm}^{2}$ & $\approx 90 \%$ & $\begin{array}{l}\text { Projected by Yole Development [8] } \\
\text { for high volume production }\end{array}$ \\
\hline Target Costs (2020s) & $\$ 10 / \mathrm{cm}^{2}$ & $80 \%$ & $\begin{array}{c}\text { Assuming } 200 \mathrm{~mm} \text { sensor wafers } \\
\text { and batch active edge process }\end{array}$ \\
\hline
\end{tabular}

\section{Technologies}

\subsection{D Circuits}

3D circuitry is the generic term for a set of technologies, including wafer bonding, thinning, and interconnect, which allows vertical interconnection of multiple layers of CMOS electronics [9] [10]. $3 \mathrm{D}$ interconnects have the advantage of reducing inductance and capacitance while increasing circuit density and allowing the integration of heterogeneous device types. The key enabling technology is the Through-Silicon-Via (TSV), a metal-filled hole etched into the wafer bulk silicon which forms the conducting path between tiers of a multi-layer assembly. In high energy physics 3D circuitry would allow us to directly integrate sensors and their readout electronics without the use of expensive and cumbersome fine pitch bump bonds.

We have explored three technologies for 3D devices. Our initial studies were with MITLincoln Labs and used their 0.18 micron three-tier process. This process utilizes oxide bonding to join the tiers and vias are etched through the SOI buried oxide after the tiers are bonded. We developed a demonstration 3-tier ILC vertex chip (VIP) in the MIT-LL process [11]. We have used 
the Ziptronix oxide bonding process with imbedded metal to mate $\mathrm{BTeV}$ FPiX ROIC wafers to sensors fabricated at MIT-Lincoln Labs [12]. Fermilab has also sponsored a two-tier 0.13 micron CMOS 3D IC run with Tezzaron/Global Foundries [13] [14] that features $1.2 \mu$ diameter, $6 \mu$ deep tungsten filled TSVs. In this process wafers were bonded face-to-face utilizing either copper thermocompression or the Ziptronix Direct Bond Interconnect (DBI) oxide bonding process described below. Bond pitch for these wafers was 4 microns. After bonding the top wafer is thinned to expose the tungsten through-silicon-vias and the top is patterned to provide contacts for bonding. The final set of wafers from Tezzaron have been received. The copper-copper wafers suffered from some alignment issues, lowering the yield of good chips, but the DBI wafers have both good alignment and yield.

Other 3D processes are becoming commercially available. IBM and Micron Semiconductor will soon announce a 3D stacked memory product based on IBM's $32 \mathrm{~nm}$ TSV process [15] with copper stud bonding shown in figure 1 . This technology is based on die-to-die bonding, potentially maximizing yield by utilizing known good die from both sensors and ROICS. Combining this process with active edge sensors could provide a very appealing path toward active tiles which could be used to build large area sensors. CEA-LETI is offering "Open 3D" services for prototyping and/or low volume production. Via-last processes, such as provided by LETI, can provide TSVs in processed wafers, with the limitation that the wafers must be thick enough to handle $(>100$ microns) and the TSV aspect ratio is generally limited to less than 20:1.

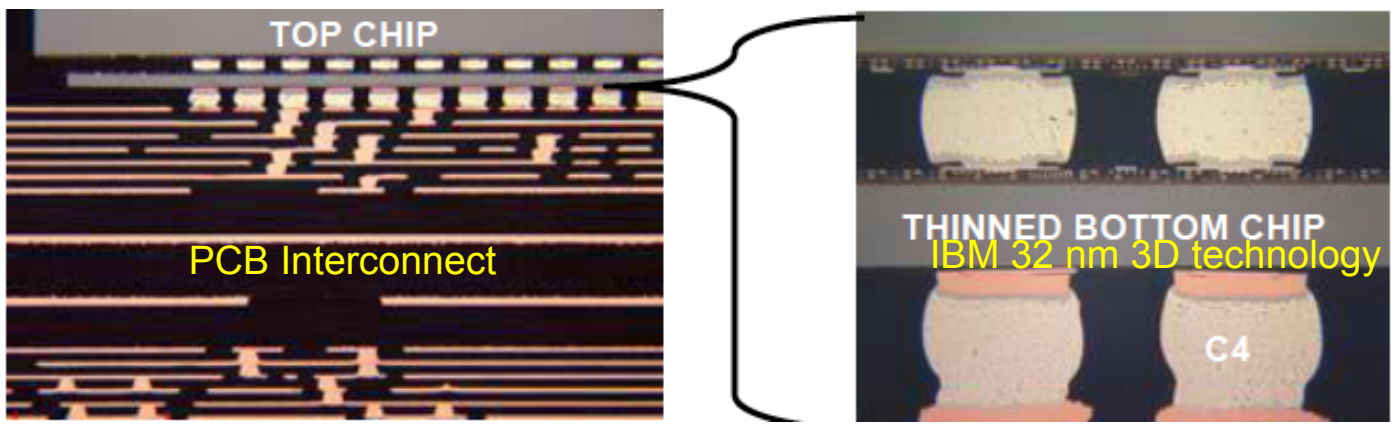

Figure 1. Cross section of a 3D assembly utilizing the IBM $32 \mathrm{~nm}$ copper stud 3D interconnect process.

\subsection{Active Edge Sensors}

Active edge sensors are an outgrowth of work done to develop 3D sensors, which provide good charge collection combined with radiation hardness. The technique utilizes a deep reactive ion etch of silicon to create a nearly vertical trench with smooth edges. The high quality of the trench wall avoids charge generation normally associated with saw-cut edges. The trenches are filled with doped polycrystalline silicon. The dopant is diffused into the surrounding single crystal silicon and annealed for activation. The dopant density gradient will make an electric field in the collection direction. These steps may be done at the same time for the other like-type electrodes. The depth of the doped silicon must be great enough so it is not depleted by the largest applied bias voltage. Mechanical integrity is maintained by bonding the sensor to a support wafer. The oxide bond also forms an etch stop for the trenching and singulation processes. In the case of an oxide bonded 
handle, in a silicon-on-insulator structure, the bond also forms an etch stop for subsequent trenching and singulation processes. An alternative active edge technology based on wafer cleaving and atomic layer deposition [16] has the prospect of achieving similar goals without the additional processing needed in the deep trench process.

\subsection{Oxide Bonding}

Bonding of silicon wafers is a key enabling technology for nanotechnology, micromachining, and 3D electronics. A variety of techniques have been developed, including adhesive bonding, metal eutectic bonding, and bonding based on the silicon oxide surface either grown or deposited on a wafer [17]. Oxide bonding has the advantages of being mechanically robust, chemically inert, and capable of withstanding the high temperatures typical of silicon processing.

The direct oxide bond [18] is formed by bringing together silicon wafers which have been planarized and chemically treated to form a hydrophilic surface. When the wafers are brought together at room temperature a van der Walls bond forms between the hydrogen atoms at the wafer surface. Further annealing above $150^{\circ} \mathrm{C}$ causes the formation of covalent hydrogen bonds and provides a substantial increase in bond strength. The Ziptronix DBI process imbeds nickel or copper in the planarized oxide surface. The metal forms an interconnect to a seed metal layer in the resulting oxide bonded wafers which can be used to build 3D interconnect structures [19]. The process requires good planarity, which is a feature of modern CMOS processes. Dust particles present during the bonding process can produce local bond voids. These unbonded areas limit the large area module yield and are a motivation for active tile development. The DBI process can be used for wafer-to-wafer or chip-to-wafer bonding. However the wafer to wafer process is least expensive and most well suited to scaling to large volumes.

\subsection{Interconnect}

Once bonded to a sensor, connections need to be made to the ROIC, which is now face down. Normally in a bump bonded pixel detector assembly the chip area is larger than that of the sensor with bond pads extending out of the edges. In an oxide bonded assembly signals as well as power need to be brought in through the bulk silicon of the ROIC. If the ROIC has imbedded through silicon bias the wafers can be thinned and vias exposed using the same technique utilized in the $3 \mathrm{DIC}$ run. A non-TSV wafer can be used by thinning the top silicon to $\approx 10 \mu$, etching that silicon to the I/O pad contacts, and depositing a redistribution metal layer to provide the final contacts through what normally would be the bottom of the ROIC. The redistribution layer can provide interconnects between adjacent chips, route power, and bump bond pads. This layer can also provide "stitching" between adjacent reticules to provide a large effective area which can be defined at the dicing stage.

An alternate interconnect approach is to utilize so-called 2.5D interconnect. This technology utilizes a silicon or glass interposer which incorporates TSVs and redistribution of signals. This approach decouples the $3 \mathrm{D}$ processing from the sensors and readout chips and offers a substrate with very fine interconnect capability and coefficient of thermal expansion match to the silicon ROICs and sensors. An interposer-based solution could partially decouple sensor and ROIC pitches, allow for a variety of external interconnects, and allow for conventional bump bonding of ROICS. 


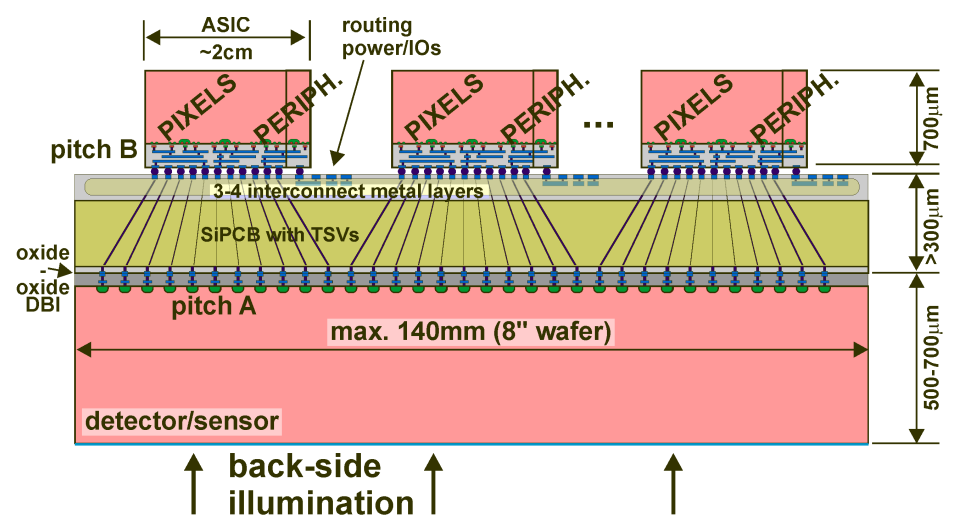

Figure 2. Sketch of an interposer-based large area array.

\section{Large Area Assembly}

We now consider techniques for the fabrication of large area arrays utilizing the tools described in section 2. All are based on wafer-to-wafer bonding of sensors and ROICs utilizing the DBI process.

\subsection{Tiles with Active Edge Sensors}

We are currently exploring a process which utilizes "standard" active edge sensors. These are n-onp devices with 200 micron thick sensors in an SOI stack with a 500 micron thick handle wafer. The tiles are fabricated by DBI bonding the sensor wafer stack to a dummy ROIC which rearranges the signal contact pattern. After bonding contacts on the dummy ROIC wafer are exposed by grinding and etching away the dummy wafer silicon substrate and first layer of oxide to expose what would normally be the bottom surface of the readout pads. This surface is then metalized to provide sites for bump bond placement. The stack is shown in figure 3. Finally the active die must be singulated. This is accomplished by etching the 10 microns of dummy wafer silicon and 3 microns of oxide, followed by an etch of the 200 microns of polysilicon filling the trench. The SOI oxide forms an etch stop in this process. The wafer is then attached to a temporary handle wafer and the original SOI handle is ground away, leaving isolated tiles.

\subsection{Post-Processed Tiles}

A simpler variant on the above process would DBI bond ROIC and untrenched sensor wafers. The tiles are then defined by etching trenches in the backside of the sensor wafer. The etching process is low temperature and should not harm the ROIC. However the resulting edges would not have the doping that normally defines the side electrode in active edge devices. Post-trench doping and annealing would not be possible, since the anneal would require higher temperatures than could be tolerated by the ROIC. In this case a process similar to that explored for slim-edge fabrication, utilizing atomic layer deposition to activate the edges should provide acceptable performance. This process avoids the SOI stack needed for the initial sensor wafer, provides a more planar surface for oxide bonding, and avoids the complex singulation and handle wafer removal necessary with the pre-processed active edge devices. 


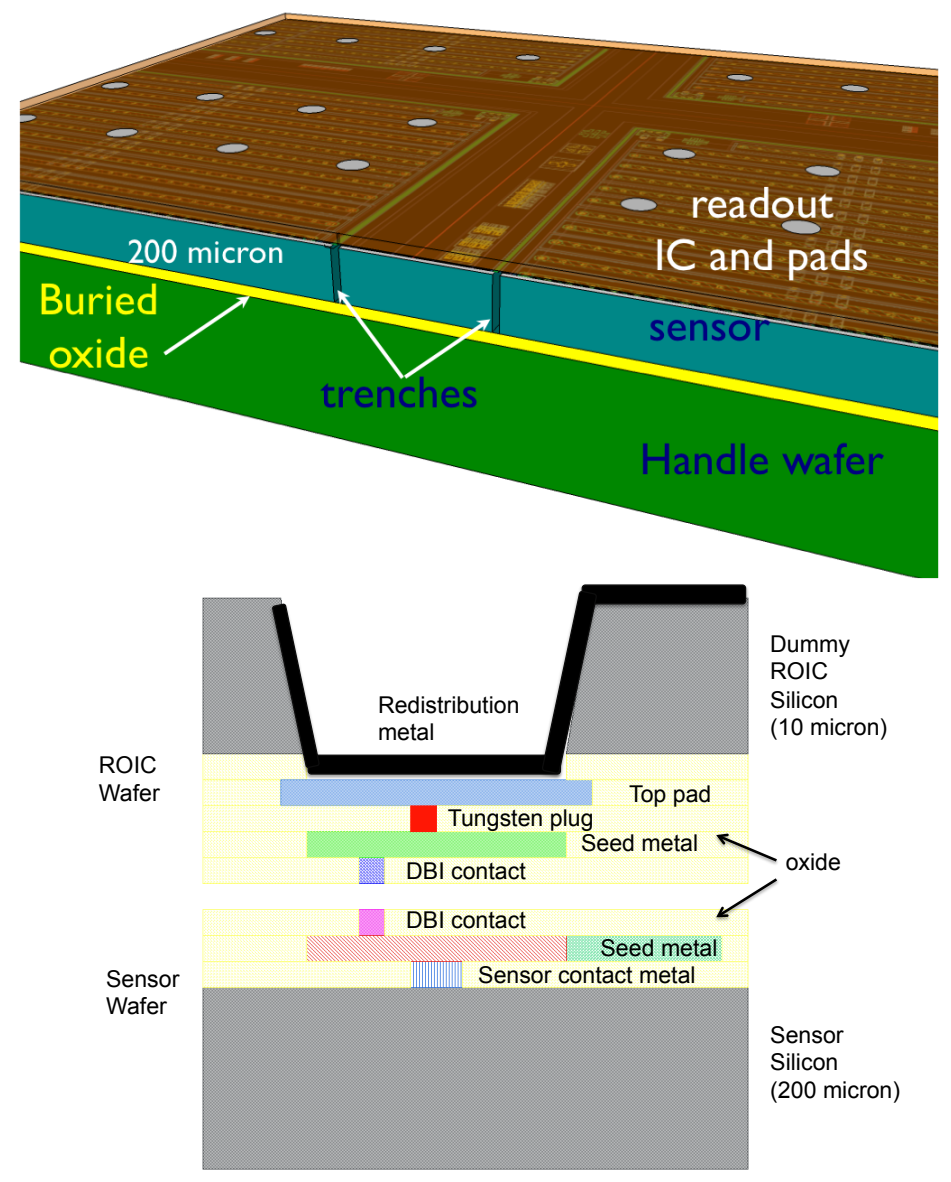

Figure 3. Top - 3D sketch showing a view of the wafer stack, including handle wafer, trenches, and the region between reticules. Bottom- Schematic view of the final stack with DBI contact layers and sensor and top contacts.

This process is very similar to plasma dicing, which utilizes the same deep reactive ion etching process to dice wafers [20], so it should be commercially available for both $200 \mathrm{~mm}$ and $300 \mathrm{~mm}$ diameter wafers.

\subsection{Die-to-Die tiles}

An alternative which utilizes the die-to-die capability of an IBM-style process could bond active edge sensor tiles to ROICs with imbedded TSVs. This probably would not require ROIC and sensor wafers of equal diameter assuming the copper bond preparation process was available for the smaller diameter sensor wafers. Although this process might not have the economies of scale of a wafer level process, improved yields by utilizing tested die might make this process competitive in cost.

\subsection{Interposer-based Array}

If the density of voids in the DBI bonded assembly is sufficiently small an appealing assembly 
would utilize a silicon interposer DBI bonded to the sensor wafer. Such an assembly could, in principle, utilize the largest sensor that can be inscribed in the wafer. The interposers redistributes the signals to ROIC bump bonds on the top of the interposer. This works well in a situation where the area of the ROIC is smaller that that of the associated pixels.

\section{Prospects}

The ability to build large areas of pixelated arrays with complex readout electronics will be crucial to future detectors. Bonding costs and yield will define the limitations of these detectors. We have described a plan to demonstrate pixelated tiles or large area arrays that have the prospect of making such large area devices affordable. To complete this development sensor wafers which match the 8" diameter of the ROIC wafers are needed. Such wafers have been demonstrated in the SOI process by both Lapis/OK [21] and American Semiconductor. We expect that commercial manufacturers will soon establish 8 " high resistivity sensor production.

\section{References}

[1] C. J. Kenney, J. D. Segal, E. Westbrook, S. Parker, J. Hasi, C. Da Via, S. Watts and J. Morse, Active-edge planar radiation sensors, Nucl. Instrum. Meth. A 565, 272 (2006).

[2] C. J. Kenney, S. I. Parker, and E. Walckiers, Results from 3D sensors with wall electrodes: near-cell-edge sensitivity measurements as a preview of active-edge sensors, IEEE Trans. Nucl. Sci. 48 2405-2410 (2001).

[3] Cinzia Da Via, Mario Deile, Jasmine Hasi, Christopher Kenney, Angela Kok, Sherwood Parker, Stephen Watts, et al., 3D Active Edge Silicon Detector Tests with $120 \mathrm{GeV}$ Muons, IEEE Trans. Nucl. Sci. 56505 (2009).

[4] M.Garcia-Sciveres,et. al.,TheFE-I4 pixel readout integrated circuit, Nucl. Instrum. Meth. A 636, 155 (2011).

[5] Current cost for 0.18 micron CMOS wafers with through-siicon vias.

[6] M. Barbero, Vertex 2011 Workshop, Rust - Austria, June 19th - 24th 2011.

[7] P. Merkel et. al. Nucl. Instrum. Meth. A 582771 (2007).

[8] Solid State Technology. http://www.electroiq.com/articles/sst/2008/10/ ziptronix-joins-low-cost-quest-for-true-3d-ic.html

[9] IBM Journal of Research and Development, Volume 52, No. 6, 2008. Issue devoted to 3D technology.

[10] Philip Garrou, Christopher Bower, Peter Ramm, Handbook of 3D Integration Technology and Applications of 3D Integrated Circuits, Wiley-VCH, 2008.

[11] G. Deptuch, M. Demarteau, J. Hoff, F. Khalid, R. Lipton, A. Shenai, M. Trimpl and R. Yarema et al., Vertically integrated pixel readout chip for high energy physics, FERMILAB-CONF-11-020-PPD.

[12] Z. Ye, in Proceedings of the International Linear Collider Workshop (LCWS08 And ILC08) 2008, edited by M. Barone, Y. Torun, N. Varelas, arXiv:0902.2801v1 .

[13] G. Deptuch, M. Demarteau, J. Hoff, R. Lipton, A. Shenai, M. Trimpl, R. Yarema and T. Zimmerman, Vertically Integrated Circuits at Fermilab, IEEE Trans. Nucl. Sci. 57, 2178 (2010). 
[14] R. Yarema, The First Multiproject Wafer Run With Chartered/Tezzaron, 2011 Front End Electronics Meeting Bergamo Italy, https://indico.cern. ch/getFile.py/access?contribId= 34 \&session $I d=1$ \&res $I d=1$ \&material $I d=s l i d e s \& \operatorname{conf} I d=122027$

[15] M. Shapiro, AIDA-Academia Meets Industry, https: / / indico.cern.ch/ contributionDisplay $\cdot$ py?session $I d=5 \&$ contribId=38\& conf Id=209454

[16] R Mori et. al., Charge collection measurements on slim-edge microstrip detectors, JINST 7 P05002 2012.

[17] Q.-Y. Tong and U. GŽsele The Electrochemical Society. ed. Semiconductor Wafer Bonding: Science and Technology (1 ed.). Wiley-Interscience. ISBN 978-0-471-57481-1 1998.

[18] Q.-Y. Tong and E. Schmidt and U. GŽsele and M. Reiche. Hydrophobic silicon wafer bonding. Applied Physics Letters 64 (5): pp. 625-627 1994.

[19] P. Enquist, G. Fountain, C. Petteway, A. Hollingsworth, and H. Grady. Low Cost of Ownership scalable copper Direct Bond Interconnect 3D IC technology for three dimensional integrated circuit applications. In: 3D System Integration, 3DIC 2009;

[20] Wei-Sheng Lei, Ajay Kumar, and Rao Yalamanchili, Die singulation technologies for advanced packaging: A critical review J. Vac. Sci. Technol. B 30, 040801 (2012)

[21] Y. Onuki, H. Katsurayama, Y. Ono, H. Yamamoto, Y. Arai, Y. Fujita, R. Ichiyama and Y. Ikegami et al., SOI detector developments, PoS VERTEX 2011, 043 (2011). 


\title{
3D Technologies for Large Area Trackers
}

\author{
G. Deptuch ${ }^{a}$, U. Heintz ${ }^{b}$, M. Johnson ${ }^{a}$, C. Kenney ${ }^{d}$, R. Lipton ${ }^{a}$, M. Narian ${ }^{b}$, S. \\ Parker $^{e}, \mathbf{A}$. Shenai ${ }^{a}$, L. Spiegel ${ }^{a}$, J. Thom ${ }^{c}$, and Z. Ye ${ }^{f}$ \\ ${ }^{a}$ Fermilab, \\ P.O. Box 500, Batavia, Il , USA \\ ${ }^{b}$ Brown University, \\ Providence, RI , USA \\ ${ }^{c}$ Cornell University, \\ Ithaca, NY, USA \\ d SLAC National Accelerator Laboratory \\ Menlo Park, CA, USA \\ ${ }^{e}$ University of Hawaii, \\ Honolulu, HI, USA \\ ${ }^{f}$ University of Ilinois, Chicago, \\ Chicago, Il, USA \\ E-mail: liptonefnal.gov
}

\begin{abstract}
We describe technologies which can be developed to produce large area, low cost pixelated tracking detectors. These utilize wafer-scale 3D electronics and sensor technologies currently being developed in industry. This can result in fully active sensor/readout chip tiles which can be assembled into large area arrays with good yield and minimal dead area. The ability to connect though the bulk of the device can also provide better electrical performance and lower mass.
\end{abstract}




\section{Contents}

$\begin{array}{ll}\text { 1. Introduction } & 1\end{array}$

2. Technologies 2

2.1 3D Circuits 2

2.2 Active Edge Sensors 3

2.3 Oxide Bonding 4

2.4 Interconnect 4

3. Large Area Assembly 5

3.1 Tiles with Active Edge Sensors 5

3.2 Post-Processed Tiles 5

3.3 Die-to-Die tiles 6

$\begin{array}{lll}3.4 & \text { Interposer-based Array } & 6\end{array}$

$\begin{array}{lll}\text { 4. } & \text { Prospects } & 7\end{array}$

\section{Introduction}

In the next generation of collider experiments detectors will be challenged by unprecedented luminosity and data volumes. We will need to build large area arrays (100's of meter ${ }^{2}$ ) of highly pixelated detectors with minimal dead area and at reasonable cost. Current fine pitch bump bonding technologies require individual placement of chips on sensors followed by a solder melt cycle. Technologies which provide wafer-scale interconnect offer prospects of lower cost, finer pitch and lower mass interconnects between sensors and readout chips. In addition 3D interconnect technology allows connections through the bulk of the silicon, providing low inductance and capacitance paths for signal and power connections. Table 1 summarizes current and projected costs and yields of various bonding technologies.

In the past pixel sensor arrays have been limited in the module area by:

1. Space needed at the edges of the detectors to reduce the field near the damaged dicing cut regions. These regions act as charge emitters and can cause unacceptably large currents in the edge strips.

2. Die size of the Readout Integrated Circuit (ROIC), determined by the die yield and reticule area

3. The need to provide edge locations for wire bonds for connection to the ROIC. 
The development of active edge sensors by C. Kenney, S. Parker and collaborators has addressed the first problem [1] [2] [3]. Recent work on the large area FEI4 chip [4] has shown that a large pixel chip can be produced with good yield. Processes related to 3D electronics can solve the third problem. The combination of all three can result in a wafer scale fabrication of tiles or arrays which can be integrated into large modules with high yield and relatively low cost. Using such tiles, large area pixelated modules can be assembled with known good integrated sensor/readout die with large pitch backside bump bond interconnects.

Table 1. Current and projected costs and yields for sensor/readout integration technologies.

\begin{tabular}{|c|c|c|c|}
\hline Component & Current or projected cost & Yield & Comment \\
\hline Readout IC & $\$ 8 / \mathrm{cm}^{2}[5]$ & $65-70 \%[6]$ & $\begin{array}{l}\text { Current 3D wafer cost and } \\
\text { FEI4 prototype yield }\end{array}$ \\
\hline Active Edge Sensors & $\$ 53 / \mathrm{cm}^{2}$ & $\approx 90 \%$ & $\begin{array}{l}\text { Current cost for prototype } \\
150 \mathrm{~mm} \text { wafers }\end{array}$ \\
\hline Silicon Strip Sensors & $\$ 10 / \mathrm{cm}^{2}$ & $\approx 100 \%$ & CMS tracker costs \\
\hline $\begin{array}{c}\text { Bump bonding } \\
\text { (2007) }\end{array}$ & $\$ 213 / \mathrm{cm}^{2}$ & $98 \%[7]$ & $\begin{array}{c}\text { CMS forward pixel costs } \\
\text { Yield } \equiv<20 \text { bad bumps/chip }\end{array}$ \\
\hline $\begin{array}{l}\text { Bump bonding } \\
\text { (2012) }\end{array}$ & $\$ 62 / \mathrm{cm}^{2}$ & - & CMS forward pixel upgrade \\
\hline $\begin{array}{c}\text { Wafer scale } \\
\text { DBI bonding }\end{array}$ & $\$ 0.04 / \mathrm{cm}^{2}$ & $\approx 90 \%$ & $\begin{array}{l}\text { Projected by Yole Development [8] } \\
\text { for high volume production }\end{array}$ \\
\hline Target Costs (2020s) & $\$ 10 / \mathrm{cm}^{2}$ & $80 \%$ & $\begin{array}{c}\text { Assuming } 200 \mathrm{~mm} \text { sensor wafers } \\
\text { and batch active edge process }\end{array}$ \\
\hline
\end{tabular}

\section{Technologies}

\subsection{D Circuits}

3D circuitry (3DIC) is the generic term for a set of technologies, including wafer bonding, thinning, and interconnect, which allows vertical interconnection of multiple layers of CMOS electronics [9] [10]. 3D interconnects have the advantage of reducing inductance and capacitance while increasing circuit density and allowing the integration of heterogeneous device types. The key enabling technology is the Through-Silicon-Via (TSV), a metal-filled hole etched into the wafer bulk silicon which forms the conducting path between tiers of a multi-layer assembly. In high energy physics 3D circuitry would allow us to directly integrate sensors and their readout electronics without the use of expensive and cumbersome fine pitch bump bonds.

We have explored three technologies for 3D devices. Our initial studies were with MITLincoln Labs and used their 0.18 micron three-tier process. This process utilizes oxide bonding to join the tiers and vias are etched through the SOI buried oxide after the tiers are bonded. We developed a demonstration 3-tier ILC vertex chip (VIP) in the MIT-LL process [11]. We have used 
the Ziptronix oxide bonding process with imbedded metal to mate $\mathrm{BTeV}$ FPiX ROIC wafers to sensors fabricated at MIT-Lincoln Labs [12]. Fermilab has also sponsored a two-tier 0.13 micron CMOS 3D IC run with Tezzaron/Global Foundries [13] [14] that features $1.2 \mu$ diameter, $6 \mu$ deep tungsten filled TSVs. In this process wafers were bonded face-to-face utilizing either copper thermocompression or the Ziptronix Direct Bond Interconnect (DBI) oxide bonding process described below. Bond pitch for these wafers was 4 microns. After bonding the top wafer is thinned to expose the tungsten through-silicon-vias and the top is patterned to provide contacts for bonding. The final set of wafers from Tezzaron have been received. The copper-copper wafers suffered from alignment problems, lowering the yield of good chips, but the DBI wafers have both good alignment and yield.

Other 3D processes are becoming commercially available. IBM and Micron Semiconductor will soon announce a 3D stacked memory product based on IBM's $32 \mathrm{~nm}$ TSV process [15] with copper stud bonding shown in figure 1 . This technology is based on die-to-die bonding, potentially maximizing yield by utilizing known good die from both sensors and ROICS. Combining this process with active edge sensors could provide a very appealing path toward active tiles which could be used to build large area sensors. CEA-LETI is offering "Open 3D" services for prototyping and/or low volume production. Via-last processes, such as provided by LETI, can provide TSVs in processed wafers, with the limitation that the wafers must be thick enough to handle $(>100$ microns) and the TSV aspect ratio is generally limited to less than 20:1.

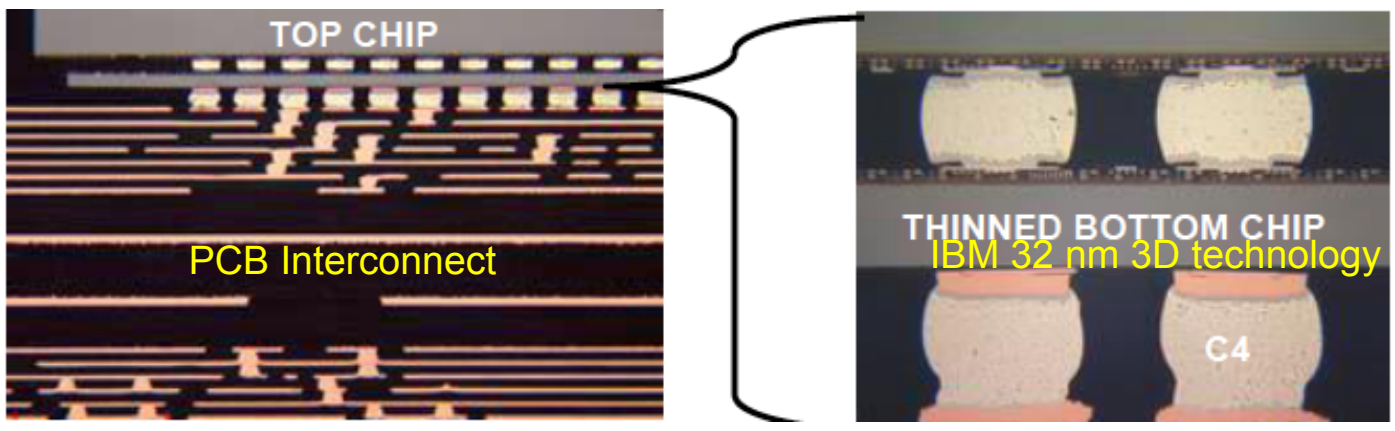

Figure 1. Cross section of a 3D assembly utilizing the IBM $32 \mathrm{~nm}$ copper stud 3D interconnect process.

\subsection{Active Edge Sensors}

Active edge sensors are an outgrowth of work done to develop 3D sensors, which provide good charge collection combined with radiation hardness. The technique utilizes a deep reactive ion etch of silicon to create a nearly vertical trench with smooth edges. The high quality of the trench wall avoids charge generation normally associated with saw-cut edges and allow closer placement of adjacent sensors. The trenches are filled with doped polycrystalline silicon. The dopant is diffused into the surrounding single crystal silicon and annealed for activation. The dopant density gradient will make an electric field in the collection direction. These steps may be done at the same time for the other like-type electrodes. The depth of the doped silicon must be great enough so it is not depleted by the largest applied bias voltage. Mechanical integrity is maintained by bonding the sensor to a support wafer. The oxide bond also forms an etch stop for the trenching and singulation 
processes. In the case of an oxide bonded handle, in a silicon-on-insulator structure, the bond also forms an etch stop for subsequent trenching and singulation processes. An alternative active edge technology based on wafer cleaving and atomic layer deposition [16] has the prospect of achieving similar goals without the additional processing needed in the deep trench process.

\subsection{Oxide Bonding}

Bonding of silicon wafers is a key enabling technology for nanotechnology, micromachining, and 3D electronics. A variety of techniques have been developed, including adhesive bonding, metal eutectic bonding, and bonding based on the silicon oxide surface either grown or deposited on a wafer [17]. Oxide bonding has the advantages of being mechanically robust, chemically inert, and capable of withstanding the high temperatures typical of silicon processing.

The direct oxide bond [18] is formed by bringing together silicon wafers which have been planarized and chemically treated to form a hydrophilic surface. When the wafers are brought together at room temperature a van der Walls bond forms between the hydrogen atoms at the wafer surface. Further annealing above $150^{\circ} \mathrm{C}$ causes the formation of covalent hydrogen bonds and provides a substantial increase in bond strength. The Ziptronix DBI process imbeds nickel or copper in the planarized oxide surface. The metal forms an interconnect to a seed metal layer in the resulting oxide bonded wafers which can be used to build 3D interconnect structures [19]. The process requires good planarity, which is a feature of modern CMOS processes. Dust particles present during the bonding process can produce local bond voids. These unbonded areas limit the large area module yield and are a motivation for active tile development. The DBI process can be used for wafer-to-wafer or chip-to-wafer bonding. However the wafer to wafer process is least expensive and most well suited to scaling to large volumes.

\subsection{Interconnect}

Once bonded to a sensor, connections need to be made to the ROIC, which is now face down. Normally in a bump bonded pixel detector assembly the chip area is larger than that of the sensor with bond pads extending out of the edges. In an oxide bonded assembly signals as well as power need to be brought in through the bulk silicon of the ROIC. If the ROIC has imbedded through silicon vias the wafers can be thinned and vias exposed using the same technique utilized in the Tezzaron two-tier 3D integrated circuit run. A non-TSV wafer can be used by thinning the top silicon to $\approx 10 \mu$, etching that silicon to the I/O pad contacts, and depositing a redistribution metal layer to provide the final contacts through what normally would be the bottom of the ROIC. The redistribution layer can provide interconnects between adjacent chips, route power, and bump bond pads. This layer can also provide "stitching" between adjacent reticules to provide a large effective area which can be defined at the dicing stage.

An alternate interconnect approach is to utilize so-called 2.5D interconnect. This technology utilizes a silicon or glass interposer which incorporates TSVs and redistribution of signals (figure 2. This approach decouples the 3D processing from the sensors and readout chips and offers a substrate with very fine interconnect capability and coefficient of thermal expansion match to the silicon ROICs and sensors. An interposer-based solution could partially decouple sensor and ROIC pitches, allowing for a variety of external interconnects, and allow for conventional bump bonding of ROICS. 


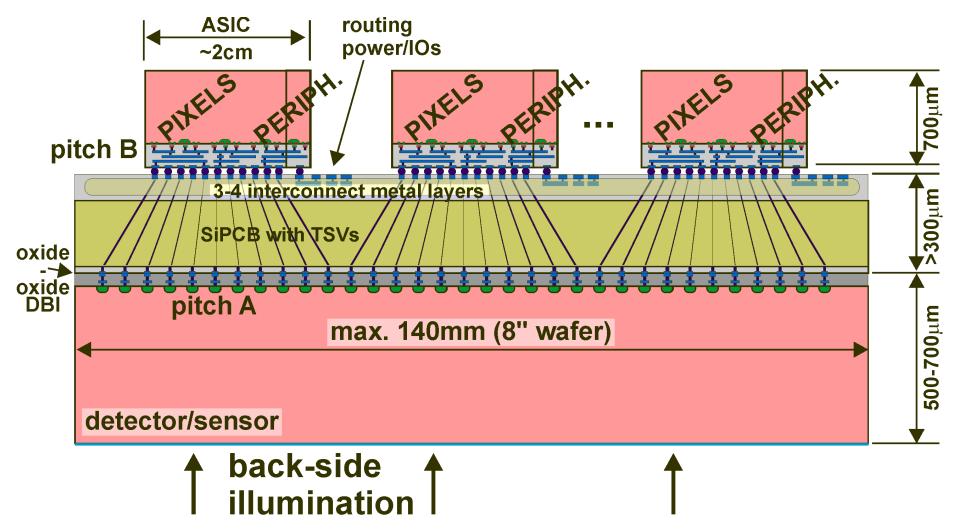

Figure 2. Sketch of an interposer-based large area array.

\section{Large Area Assembly}

We now consider techniques for the fabrication of large area arrays utilizing the tools described in section 2. All are based on wafer-to-wafer bonding of sensors and ROICs utilizing the DBI process.

\subsection{Tiles with Active Edge Sensors}

We are currently exploring a process which utilizes "standard" active edge sensors. These are n-onp devices with 200 micron thick sensors in an SOI stack with a 500 micron thick handle wafer. The tiles are fabricated by DBI bonding the sensor wafer stack to a dummy ROIC which rearranges the signal contact pattern. After bonding contacts on the dummy ROIC wafer are exposed by grinding and etching away the dummy wafer silicon substrate and first layer of oxide to expose what would normally be the bottom surface of the readout pads. This surface is then metalized to provide sites for bump bond placement. The stack is shown in figure 3. Finally the active die must be singulated. This is accomplished by etching the 10 microns of dummy wafer silicon and 3 microns of oxide, followed by an etch of the 200 microns of polysilicon filling the trench. The SOI oxide forms an etch stop in this process. The wafer is then attached to a temporary handle wafer and the original SOI handle is ground away, leaving isolated tiles.

\subsection{Post-Processed Tiles}

A simpler variant on the above process would DBI bond ROIC and untrenched sensor wafers. The tiles are then defined by etching trenches in the backside of the sensor wafer. The etching process is low temperature and should not harm the ROIC. However the resulting edges would not have the doping that normally defines the side electrode in active edge devices. Post-trench doping and annealing would not be possible, since the anneal would require higher temperatures than could be tolerated by the ROIC. In this case a process similar to that explored for slim-edge fabrication, utilizing atomic layer deposition to activate the edges should provide acceptable performance. This process avoids the SOI stack needed for the initial sensor wafer, provides a more planar surface for oxide bonding, and avoids the complex singulation and handle wafer removal necessary with the pre-processed active edge devices. 


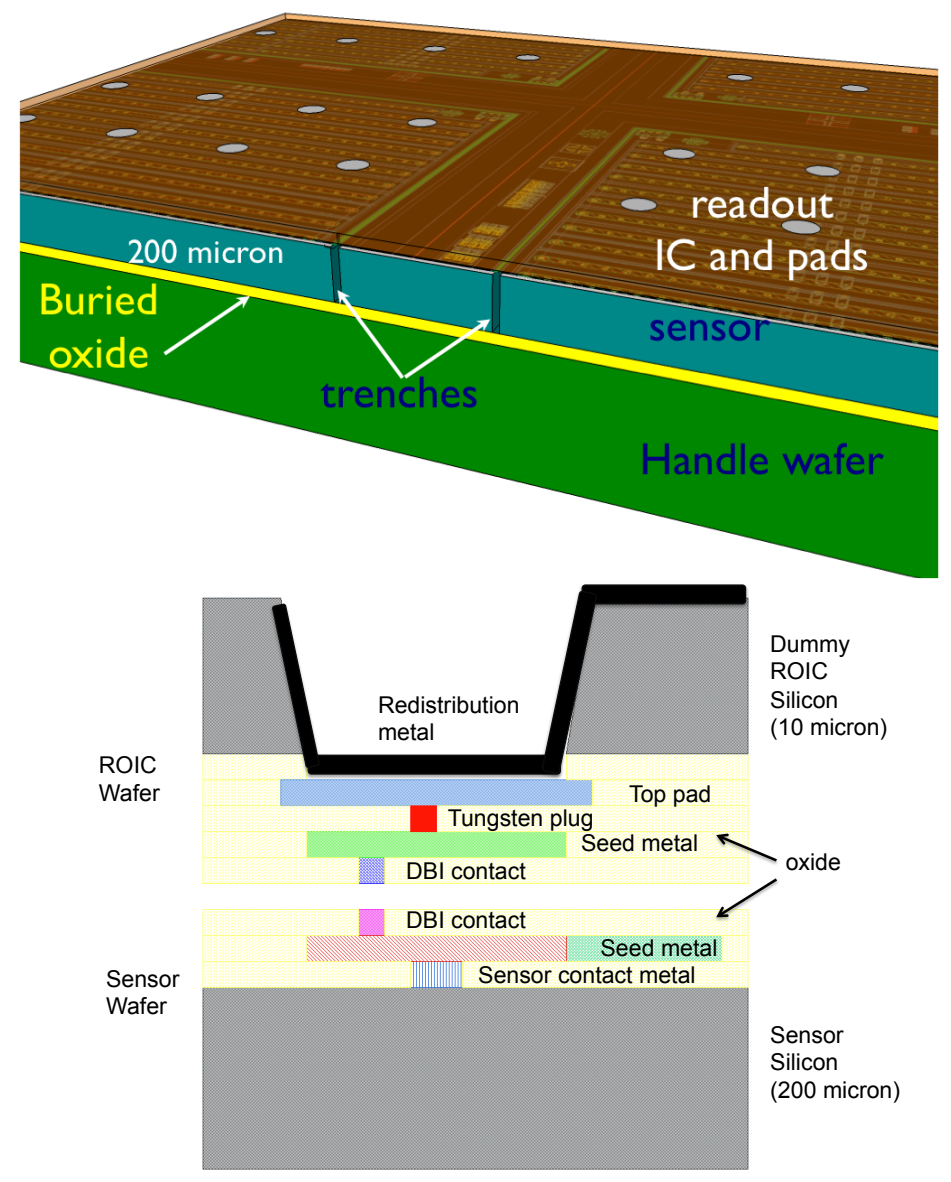

Figure 3. Top - 3D sketch showing a view of the wafer stack, including handle wafer, trenches, and the region between reticules. Bottom- Schematic view of the final stack with DBI contact layers and sensor and top contacts.

This process is very similar to plasma dicing, which utilizes the same deep reactive ion etching process to dice wafers [20], so it should be commercially available for both $200 \mathrm{~mm}$ and $300 \mathrm{~mm}$ diameter wafers.

\subsection{Die-to-Die tiles}

An alternative which utilizes the die-to-die capability of an IBM-style process could bond active edge sensor tiles to ROICs with imbedded TSVs. This probably would not require ROIC and sensor wafers of equal diameter assuming the copper bond preparation process was available for the smaller diameter sensor wafers. Although this process might not have the economies of scale of a wafer level process, improved yields by utilizing tested die might make this process competitive in cost.

\subsection{Interposer-based Array}

If the density of voids in the DBI bonded assembly is sufficiently small an appealing assembly 
would utilize a silicon interposer DBI bonded to the sensor wafer. Such an assembly could, in principle, utilize the largest sensor that can be inscribed in the wafer with good yield, typically about $1 \mathrm{~cm}$ inset from the wafer edge. The interposers redistributes the signals to ROIC bump bonds on the top of the interposer. This works well in a situation where the area of the ROIC is smaller that that of the associated pixels.

\section{Prospects}

The ability to build large areas of pixelated arrays with complex readout electronics will be crucial to future detectors. Bonding costs and yield will define the limitations of these detectors. We have described a plan to demonstrate pixelated tiles or large area arrays that have the prospect of making such large area devices affordable. To complete this development sensor wafers which match the 8 " diameter of the ROIC wafers are needed. Such wafers have been demonstrated in the SOI process by both Lapis/OK [21] and American Semiconductor. We expect that commercial manufacturers will soon establish 8 " high resistivity sensor production.

\section{References}

[1] C. J. Kenney, J. D. Segal, E. Westbrook, S. Parker, J. Hasi, C. Da Via, S. Watts and J. Morse, Active-edge planar radiation sensors, Nucl. Instrum. Meth. A 565, 272 (2006).

[2] C. J. Kenney, S. I. Parker, and E. Walckiers, Results from 3D sensors with wall electrodes: near-cell-edge sensitivity measurements as a preview of active-edge sensors, IEEE Trans. Nucl. Sci. 48 2405-2410 (2001) .

[3] Cinzia Da Via, Mario Deile, Jasmine Hasi, Christopher Kenney, Angela Kok, Sherwood Parker, Stephen Watts, et al., 3D Active Edge Silicon Detector Tests with 120 GeV Muons, IEEE Trans. Nucl. Sci. 56505 (2009).

[4] M.Garcia-Sciveres,et. al.,The FE-I4 pixel readout integrated circuit, Nucl. Instrum. Meth. A 636, 155 (2011).

[5] Current cost for 0.18 micron CMOS wafers with through-siicon vias.

[6] M. Barbero, Vertex 2011 Workshop, Rust - Austria, June 19th - 24th 2011.

[7] P. Merkel et. al. Nucl. Instrum. Meth. A 582771 (2007).

[8] Solid State Technology. http://www.electroiq.com/articles/sst/2008/10/ ziptronix-joins-low-cost-quest-for-true-3d-ic.html

[9] IBM Journal of Research and Development, Volume 52, No. 6, 2008. Issue devoted to 3D technology.

[10] Philip Garrou, Christopher Bower, Peter Ramm, Handbook of 3D Integration Technology and Applications of 3D Integrated Circuits, Wiley-VCH, 2008.

[11] G. Deptuch, M. Demarteau, J. Hoff, F. Khalid, R. Lipton, A. Shenai, M. Trimpl and R. Yarema et al., Vertically integrated pixel readout chip for high energy physics, FERMILAB-CONF-11-020-PPD.

[12] Z. Ye, in Proceedings of the International Linear Collider Workshop (LCWS08 And ILC08) 2008, edited by M. Barone, Y. Torun, N. Varelas, arXiv:0902.2801v1 .

[13] G. Deptuch, M. Demarteau, J. Hoff, R. Lipton, A. Shenai, M. Trimpl, R. Yarema and T. Zimmerman, Vertically Integrated Circuits at Fermilab, IEEE Trans. Nucl. Sci. 57, 2178 (2010). 
[14] R. Yarema, The First Multiproject Wafer Run With Chartered/Tezzaron, 2011 Front End Electronics Meeting Bergamo Italy, https://indico.cern. ch/getFile.py/access?contribId= 34 \&session $I d=1$ \&res $I d=1$ \&material $I d=s l i d e s \& \operatorname{conf} I d=122027$

[15] M. Shapiro, AIDA-Academia Meets Industry, https: / / indico.cern.ch/ contributionDisplay $\cdot$ py?session $I d=5 \&$ contribId=38\& conf Id=209454

[16] R Mori et. al., Charge collection measurements on slim-edge microstrip detectors, JINST 7 P05002 2012.

[17] Q.-Y. Tong and U. GŽsele The Electrochemical Society. ed. Semiconductor Wafer Bonding: Science and Technology (1 ed.). Wiley-Interscience. ISBN 978-0-471-57481-1 1998.

[18] Q.-Y. Tong and E. Schmidt and U. GŽsele and M. Reiche. Hydrophobic silicon wafer bonding. Applied Physics Letters 64 (5): pp. 625-627 1994.

[19] P. Enquist, G. Fountain, C. Petteway, A. Hollingsworth, and H. Grady. Low Cost of Ownership scalable copper Direct Bond Interconnect 3D IC technology for three dimensional integrated circuit applications. In: 3D System Integration, 3DIC 2009;

[20] Wei-Sheng Lei, Ajay Kumar, and Rao Yalamanchili, Die singulation technologies for advanced packaging: A critical review J. Vac. Sci. Technol. B 30, 040801 (2012)

[21] Y. Onuki, H. Katsurayama, Y. Ono, H. Yamamoto, Y. Arai, Y. Fujita, R. Ichiyama and Y. Ikegami et al., SOI detector developments, PoS VERTEX 2011, 043 (2011). 


\section{D Geometry Sensors}

Planned upgrades to the LHC will result in increasingly harsh radiation environments close to the interaction point. Existing semiconductor sensor technologies can not operate effectively over the duration of an experiment under these radiation conditions. One path to meet this challenge is through the use of 3D or non-planar geometry sensors.

Development of such sensors has been on-going since the 1990s and is still an active area of study with a growing impact beyond the Energy Frontier. The selection of 3Dgeometry sensors for the ATLAS insertable B-layer of pixels during the 2013-2014 shutdown is an indication of the promise of such technologies. Such sensors can also offer faster charge collection, when for example structured as parallel trenches, than traditional planar sensors. Fast charge-collection maybe useful for beam monitors or in time-of-flight systems.

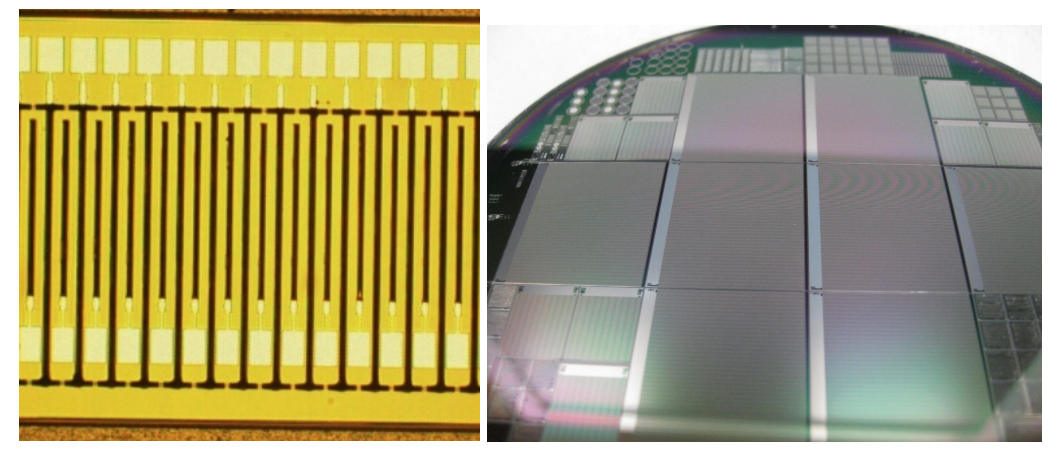

Figure 1. Fast interdigitated trenches with both $\mathrm{n}$ and $\mathrm{p}$ type readout (left) and a wafer with ATLAS FE-I4 3D sensors (right).

A significant improvement in radiation tolerance may be achievable by incorporating limited internal gain into the sensor via avalanche multiplication. This would be accomplished by performing extensive calculations to explore the device geometry, biasing, and diffusion parameter space. Prototype runs to confirm the simulation results with follow-on irradiations and beamtests to qualify the concept and final sensors would be need.

There are a number of applications for an imager, which can record the intensity of light within multiple color bands and with high quantum efficiency. One motivation is widearea optical surveys for cosmology, such as SDSS, DES, LSST, and JDEM, all utilizing silicon optical sensors, CCDs or CMOS imagers combined with optical filters to determine some information about the spectral of galaxies. SDSS uses five optical filters and so for each field of view takes five separate images. One important use of this kind of spectral information, relevant for studies in cosmology, is to determine the redshift or z of distant galaxies using the photometry of the five colors (photo-z). Of course, the need to take multiple images of the same field, each with a narrow-band filter, reduces the throughput of the telescope by the number of colors. This factor could be partially recovered with an optical sensor sensitive to color, where each pixel consisted of multiple color bands and multiple readout channels. Such a sensor would be equivalent to increasing the aperture of the telescope by a factor of 4 or 5 for instance. The significant benefits of such an increase make a color-sensitive sensor very attractive. 
The absorption coefficient for visible light in silicon is highly wavelength dependent, so a color-sensitive optical sensor is possible if each pixel is segmented along the depth of the silicon and individually read out. A possible arrangement with 3 layers is shown in Figure 2.

At present 3D-geometry sensors offer a viable way forward to meet the challenges of vertexing in the ultra-high-luminosity era and obtaining Photo-z measurements in largescale optical telescope surveys in astrophysics. More particle physics applications of nonplanar geometry sensors and semiconductor micro-machining doubtless await.
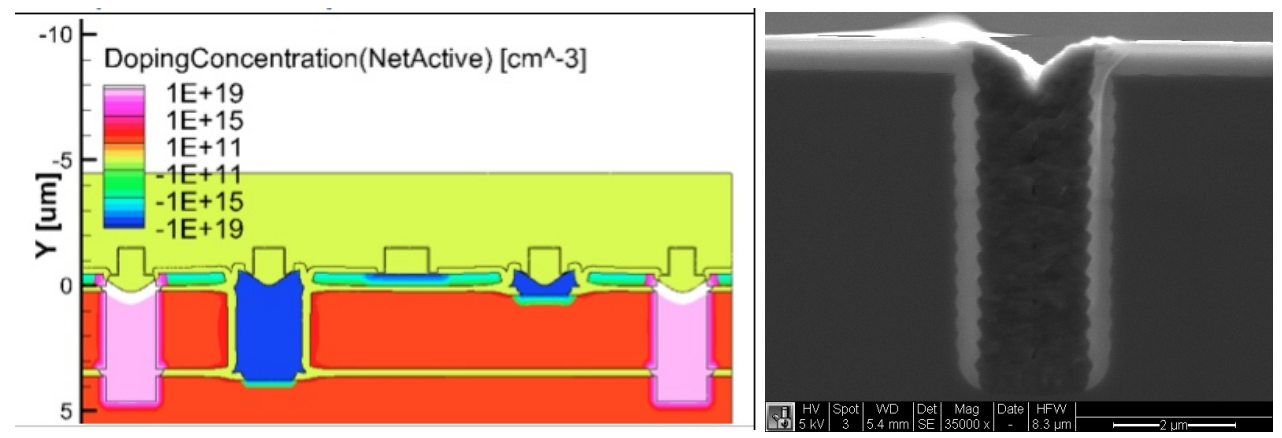

Fig.2 Process simulation for a poly-chromic CCD (left), via with bottom contact and sidewall dielectric for multi-layer CCD (right). 


\title{
Use of Flat Panel Microchannel Plates in Sampling Calorimeters with Timing
}

\author{
Anatoly Ronzhin (Fermilab) and Henry Frisch (EFI, Univ. of Chicago)
}

Electron multipliers were first proposed as the active elements in calorimeters in 1990 [1]. The attractive properties of such calorimeters are: 1) a 2-dimensional map of energy at each sampling depth; 2) timing information at each depth; and 3) possible separation of electromagnetic and hadronic energy based on the differences in timing and spectrum of Cherenkov and scintillation light [2,3].

An electron-multiplier-based sampling calorimeter requires thin planar detectors at an affordable cost. Large-area photodetectors based on ALD-functionalized 20-cm-square borosilicate glass capillary micro-channel plates (MCPs), currently being developed by the LAPPD Collaboration [4], are candidates for the active elements.

Here we propose using large-area MCPs assembled without the usual bialkali photocathodes as the active element in sampling calorimeters, as described in Ref. [1]. LAPPD modules without photocathodes can be economically assembled in a glove box and then pumped and sealed using the process to construct photomultipliers, bypassing the slow and expensive vacuum-transfer process required by bialkali photocathodes[5].

The first use of electron multipliers, in this case MCPs, as an active element in multi-layer sampling calorimeters is described in Ref. [1]. A chevron of two MCPs in vacuum (left-hand panel of Fig. 1) was located behind a tungsten plate on which $5 \mathrm{GeV}$ and $26 \mathrm{GeV}$ electrons were incident as shown in the middle panel of Fig. 1. The amplitude of the MCP signal (right-hand panel) as a function of the tungsten thickness was measured. The primary electrons produce a shower of secondary particles, including positron-electron pairs and gammas; the response of the MCPs is proportional to the number of particles in the shower, which is dependent on the initial particle energy.
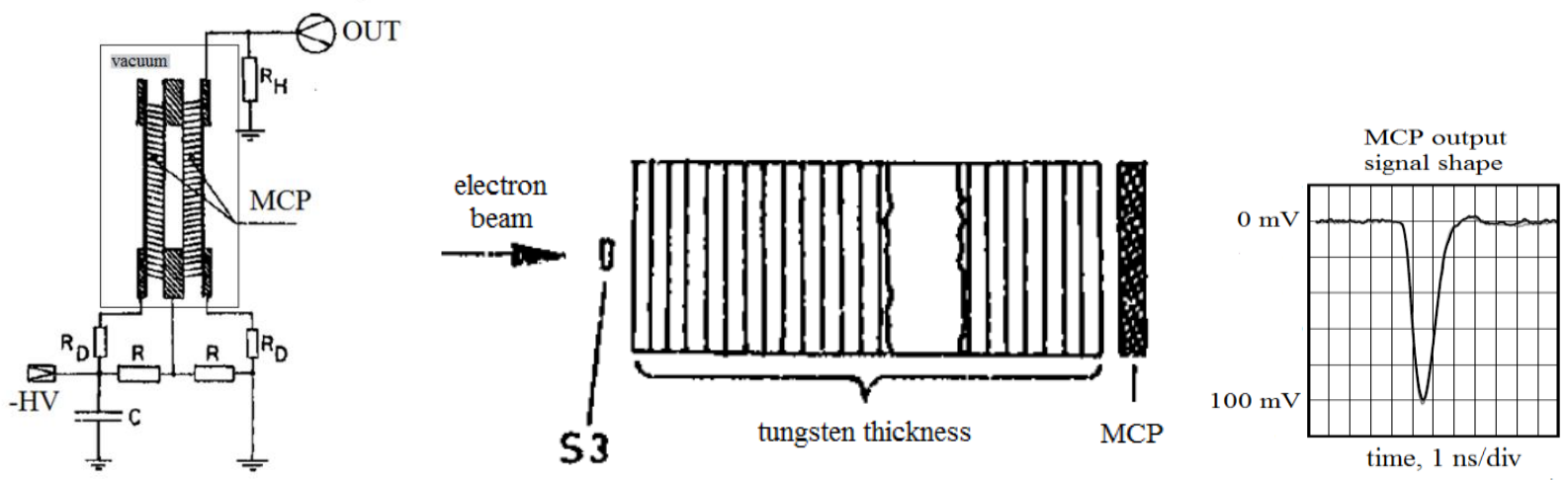

Fig 1. The electrical schematic of the tested device based on 2 MCPs (Left), the beam setup (Middle) and a typical signal trace (Right) . The output signal is very short ( 2.5 ns). The signal amplitude depends on the thickness of the tungsten absorber. 

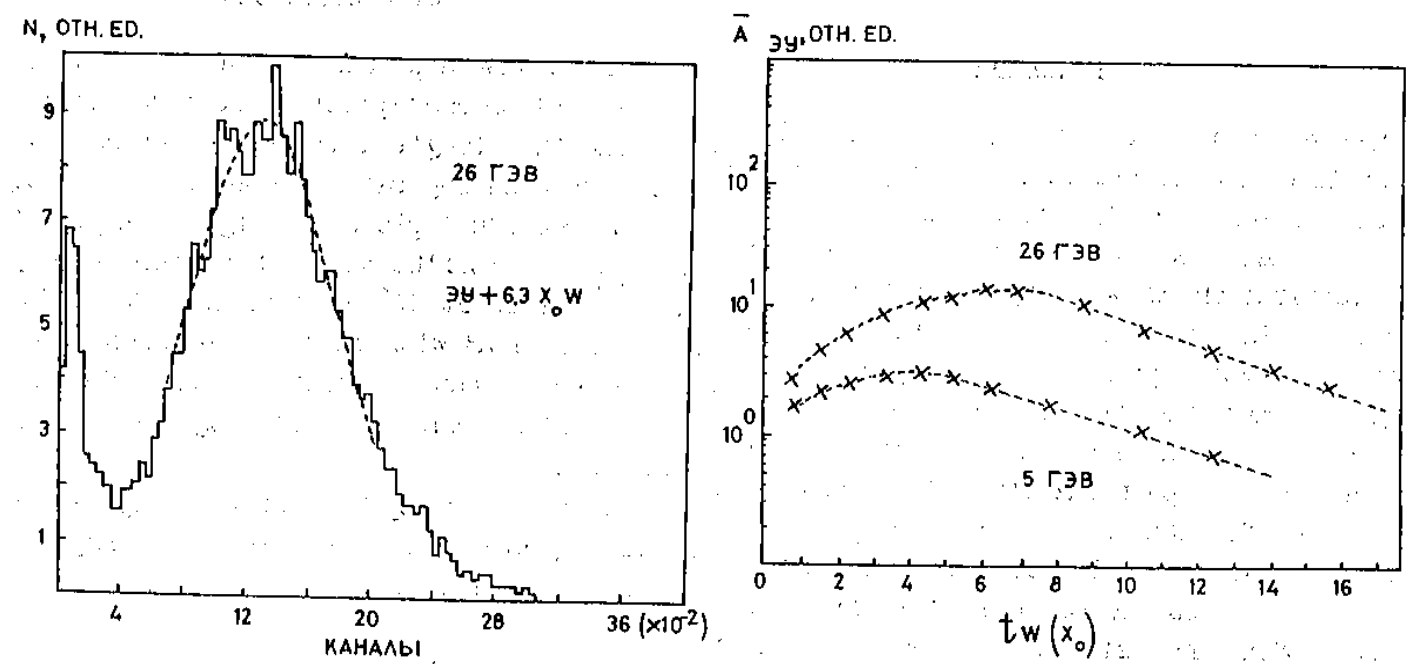

Fig 2. Left: the measured pulse height spectrum from the MCP chevron pair for $26 \mathrm{GeV}$ electrons after 6.3 radiation lengths of tungsten absorber. Right: the measured shower profile for 26 (top) and $5 \mathrm{GeV}$ (bottom) electrons versus depth in radiation length (from Ref. 1).

We propose to investigate the possibility of producing an electromagnetic calorimeter based on $\mathrm{W}$ and $\mathrm{Pb}$ absorber plates sandwiched with LAPPD detectors. Measurements can be made with bare plates, Cherenkov radiators, and/or scintillator. The LAPPD Collaboration has developed a thin, flat, system package that provides both 2D energy maps and correlated timing, including a vertical slice of a complete DAQ system [6]. Tests will be made with a fast laser, radioactive sources, and test beams at Fermilab and/or SLAC. If the results are promising we would like to construct and test a full-size electromagnetic calorimeter module large enough to contain high-energy showers, although this will require investment in additional chips, boards, and person-power.

We thank our all our colleagues in LAPPD, with special thanks to Joe Gregar of the ANL glass shop for his superlative glass work, on which this development depends.

Bibliography:

1. A. A. Derevshchikov, V. Yu. Khodyrev, V.I. Kryshkin, V.E. Rakhmatov, A. I. Ronzhin, "On possibility to make a new type of calorimeter: radiation resistant and fast". Preprint IFVE 90-99, Protvino, Russia, 1990.

2. H. J. Frisch, "The Development of Large-Area Thin Planar Psec Photodetectors", IEEE Nuclear Science Symposium and Medical Imaging Conference, Knoxville Ky, Oct. 2010

3. Frisch H. J.; Genat, J-F; Grabas,H; Kao, C-M; Chen, C-T; Kim, H. ; Tang, F; Pending US Patent Application 13/044,442 USE OF FLAT PANEL MICROCHANNEL PHOTOMULTIPLIERS IN SAMPLING CALORIMETERS WITH TIMING

4. The LAPPD Collaboration: see http://psec.uchicago.edu. The link to the Library leads to links to the Document, Figure, and Image Libraries, as well as Workshops on Fast Timing, Photocathodes, and applications.

5. The highly-reactive nature of bialkali photocathodes precludes exposure to air.

6. E. Oberla, "A Fast Waveform Digitization ASIC-based DAQ for a Position- and Time-Sensing Large Area Photodetector System, PHOTDET2012, LAL Orsay, June 2012. 
Title: Combination of Active Edge and 3D Electronics Technologies

Authors: R. Lipton, C. Kenney, S. Parker, L. Spiegel, J. Thom

Time Frame: Intermediate

Physics Justification: Future detectors for LHC, lepton colliders, and Intensity Frontier experiments will require large areas $\left(200 \mathrm{~m}^{2}\right.$ in CMS) of highly pixelated sensors with pixel sizes as small as $20 \times 20$ microns. These sensors must be low cost, have low mass and low power dissipation. Current technologies are too expensive and specialized for deployment in such large area systems.

Technical Capabilities: The natural size of a pixelated silicon detector with CMOS integrated circuit readout is set by the size of the IC reticule, usually about $2 \times 3 \mathrm{~cm}$. Larger area devices must be fabricated with arrays of these ICs bonded to a larger sensor. Sensor edges must typically have dead regions for field shaping 2-3 times the sensor thickness. This limits the tiling of sensors with standard edge processing. There are several problems with this approach:

- Access to interconnects is typically only be wirebonds at the periphery. This limits the layout of the chip arrays to two columns. It also requires significant peripheral dead area needed for wirebonded interconnects and for power and signal busses on the base board.

- Fine pitch bump bonds are expensive. Solder bump pitch is typically limited to a few hundred microns.

- There is a placement yield for each IC, so for a sensor tiled with 25 readout chips the overall yield may be unacceptable. This is especially true if unconventional bonding techniques are used which may not be reworkable.

Technical Requirements: 3D technologies being developed by the IC industry promise low cost, very fine pitch (4 micron) wafer-scale bonding technologies. 3D circuit technology with through-silicon vias allow all contacts to be made through the backside with coarse pitch bump bonds, eliminating area lost to bond pads. We can combine this wafer-scale bonding technology with with active edge sensor technology developed for 3D sensors to form 4 side buttable tiles. Active edges are fabricated utilizing Deep Reactive Ion Etch technology (DRIE) to provide smooth, defect free, sensor edges which limit the leakage currents due to fields that extend to the sensor edge. Such tiles could be individually tested to decouple IC device and placement yields for large area modules.

We are currently in the process of a demonstration of this technology utilizing 200 micron thick sensor wafers fabricated on a Silicon-on-Insulation (SOI) stack by VTT. These sensors will be bonded using the Ziptronix Direct Bond Interconnect (DBI) wafer bonding technology to a dummy readout wafer being fabricated at Cornell. Ziptronix will thin the resulting stack to expose backside pads for bump bonding. The resulting stack will be singulated at SLAC and tested for efficiency as a tiled matrix.

Industrial Involvement: Initial R\&D utilizes wafer-to-wafer DBI oxide bonding technology pioneered by Ziptronix. Full scale utilization of these technologies will require sensor wafers which match the 8 " diameter integrated circuit wafers. Infineon is currently developing such 
wafers. The DRIE for singulating these tiles will be developed at SLAC and could be industrialized at a company such as Novati, which has 8" MEMS capabilities and could also fabricate the sensor wafers.

Key Motivations for this Detector R\&D: Lower cost, finer pitch, more efficient pixelated arrays.

\section{References:}

1. R Lipton et. al., Combining the two 3Ds, JINST 7 C12010 2012

2. C.J. Kenney et al. 2006 Active-edge planar radiation sensors, Nucl. Instrum. Meth. A 565272

3. Q.-Y. Tong, E. Schmidt, U. Gösele and M. Reiche 1994 Hydrophobic silicon wafer bonding, App. Phys. Lett. 64625

4. Z. Ye, 2008 Sensor/ROIC Integration using Oxide Bonding, in proceedings of the International Linear Collider Workshop (LCWS08 And ILC08), M. Barone, Y. Torun and N. Varelas eds., [arXiv:0902.2801] 


\section{Diamond Sensors}

\section{Harris Kagan (OSU), William Trischuk (Toronto)}

Luminosity monitors, beam monitors and tracking detectors in future collider experiments, including LHC upgrades must operate in radiation environments several orders of magnitude harsher than those of any current detector. As the environment becomes harsher detectors not segmented, either spatially or in time, become challenged to separate signal from background. Present tracking detectors close to the interaction region are based on highly segmented silicon sensor technology. Chemical Vapour Deposition (CVD) diamond has a number of properties that make it an attractive alternative for high-energy physics detector applications. Its large band-gap (5.5 eV) and large displacement energy (42 $\mathrm{eV} /$ atom) make it a material that is inherently radiation tolerant with very low leakage currents and can be operated near room temperature simplifying the mechanical support and cooling necessary. The drawback of using diamond is that it only provides $60 \%$ of the number of charge carriers, per unit radiation length of thickness, relative to silicon. However the steady luminosity increase at colliders, in particular at the LHC, justify considering diamond as a replacement for $\mathrm{Si}$ in the innermost layer(s) of the vertex trackers. CVD diamond is being investigated by a number of groups for use very close to LHC interaction regions, where the most extreme radiation conditions are found. Here we describe the requirements for inner tracking sensors at future colliders, highlight the R\&D on diamond sensors underway and conclude with a discussion of scalability and cost.

\begin{tabular}{|c|c|c|}
\hline In order to continue the & Requirement & Specification \\
\hline physics studies at the LHC & Sensor size & $5 \times 5 \mathrm{~cm}^{2}$ \\
\hline it is imperative that & Sensor Thickness & 400 microns \\
\hline precision tracking continue & Minimum charge mean free path & 250 microns \\
\hline to be present at the & Minimum average charge before irradiation & 9000 electrons \\
\hline $\begin{array}{l}\text { smallest possible radii. In } \\
\text { practice, this means the }\end{array}$ & $\begin{array}{l}\text { Minimum mean free path/ } \\
\text { charge after } 10^{16} \mathrm{~cm}^{-2}\end{array}$ & $\begin{array}{l}150 \text { microns / } \\
5400 \text { electrons }\end{array}$ \\
\hline sensors must continue $\mathrm{t}$ & Minimum signal/threshold after $10^{16} \mathrm{~cm}^{-2}$ & 3 \\
\hline operate effici & Single hit efficiency & $>99.9 \%$ \\
\hline fluences & Spatial resolution & $<14 \mu \mathrm{m}$ \\
\hline & Maximum operating voltage & $1000 \mathrm{~V}$ \\
\hline & Maximum total leakage current (@1000 V) & $100 \mathrm{nA}$ \\
\hline
\end{tabular}

tracker is paramount as Table 1: Sensor material requirements for inner-most layers of final LHC trackers. excess scattering and

pair conversion significantly compromise pattern recognition and further exacerbates occupancies. Diamond sensors do not require extensive cooling and, being low Z, present less material per unit thickness than silicon or other solid-state tracking media. Of course, to be viable it must be possible to pixelate the diamond sensors, read them out with integrated VLSI electronics and produce reasonably large arrays affordably. Table 1 shows a list of sensor requirements that would make diamond a viable alternative for future energy frontier trackers. In what follows we describe the R\&D aimed at demonstrating that diamond is a viable sensor material for these applications. Although diamond sensors have recently been targeted at collider detector applications, their first use in HEP was in Bfactory background monitoring. They continue to be good candidates for beam diagnostics at intensity frontier machines. 
Significant progress in diamond sensor R\&D has been made over the last five years as the LHC experiments have come on line. All four LHC experiments include diamond beam monitors to protect them from un-stable beam conditions. The most sophisticated of these, in the ATLAS experiment, are also used as the default luminosity counters providing collision and background counting rates that have been stable to better than $1 \%$ over the first three years of LHC operation. Reading out the diamond with sub-ns shaping-time electronics has been crucial to achieving this stability in ATLAS as it allows a clear distinction between signals in-time with bunch crossings and background particles that arrive out-of-time. The RD42 collaboration has performed extensive irradiations of CVD diamond material with protons ranging from $26 \mathrm{MeV}$ to $24 \mathrm{GeV}$, pions at $300 \mathrm{MeV}$ and reactor neutrons. Figure 1 shows the change of carrier mean free path (signal produced when MIPs traverse the sensor) in state-of-the-art CVD diamond material (typical mean free paths of 300 microns in pCVD material) showing superior radiation tolerance to fluences

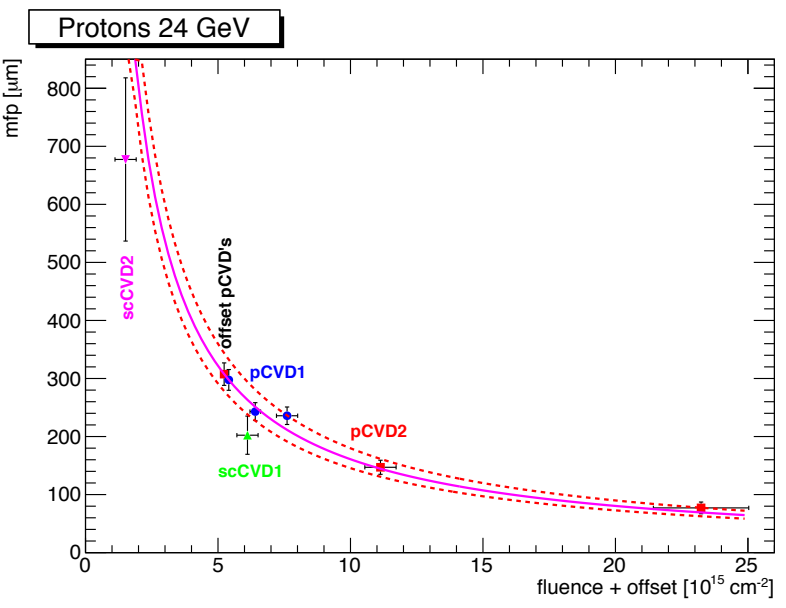

Figure 1: Mean free path of charge carriers in CVD diamond sensors vs. fluence of $24 \mathrm{GeV}$ protons. All types follow a common degradation due to the appearance of additional traps. pCVD material is offset to reflect the level of traps in its as-grown state. beyond $10^{16}$.

Over the last five years there has also been good progress on scaling up the growth of CVD diamond material. Several manufacturers have demonstrated the capability of growing wafers $10 \mathrm{~cm}$ in diameter (or larger) with charge collection distances that are suitable for single MIP tracker applications. As a result prices have started to come down although they still remain higher than for silicon sensors although silicon sensors that might survive the dose necessary to survive in future LHC pixel detectors are more expensive due to the elaborate processing they require. In addition to learning how to grow the materials manufacturers have learned that surface preparation is important to the overall quality of the sensors and the long-term survival of electrical contacts to the resulting sensors. The CMS experiment is preparing a set of forward pixel telescopes, based on the largest single crystal CVD diamond sensors currently available $\left(0.5 \times 0.5 \mathrm{~cm}^{2}\right)$ for installation during the current LHC machine stop. In turn ATLAS is preparing a pixel Diamond Beam Monitor using larger poly-crystalline CVD diamond sensors $\left(1.8 \times 2.1 \mathrm{~cm}^{2}\right)$ on a similar timescale. Both of these projects have demonstrated routine

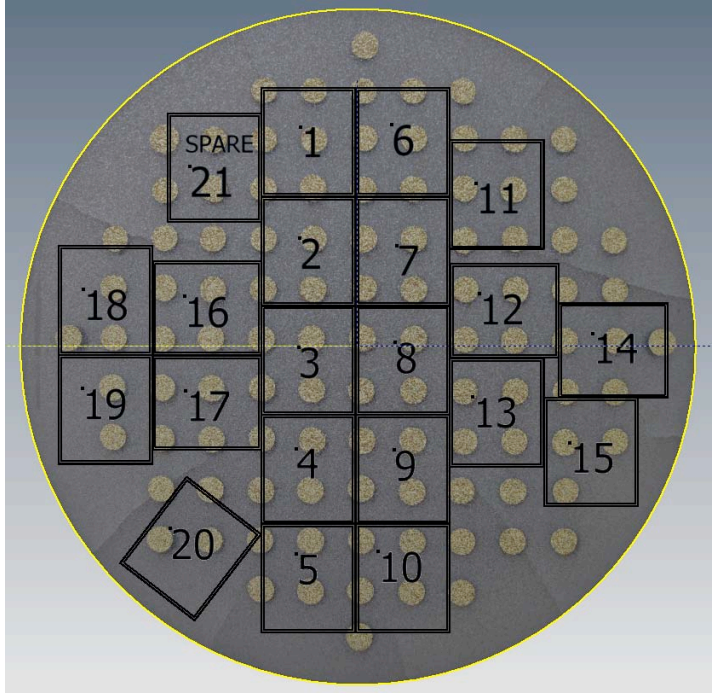

Figure 2: A poly-CVD diamond wafer from II-VI Inc. for the ATLAS Diamond Beam Monitor, showing 21 sensor substrates prior to dicing. 
large-scale bump-bonding of VLSI pixel readout chips to diamond sensors and aim to demonstrate routine tracking in the most demanding environment yet explored, less than 5 $\mathrm{cm}$ from the LHC beamline and $1 \mathrm{~m}$ from their, respective, LHC interaction points. A number of studies have been performed to characterise the tracking performance with diamond sensors. Charge sharing has been demonstrated with the diamond strip trackers giving position resolutions of better than $9 \mu \mathrm{m}$ [1] and similar resolution has been seen in $50 \mu \mathrm{m}$ wide pixels in small single crystal pixel devices [2]. Resolution has been shown to improve in polycrystalline CVD sensors after irradiation as the introduction of defects improves the material uniformity [3].

The development of diamond sensors for HEP applications has piggy backed on industrial production capabilities for other applications. CVD substrates have applications in thermal management, X-ray windows and, potentially, dosimetry (with Carbon being a better match for human tissue than other solid-state sensor materials). Over the past ten years the RD42 collaboration has worked with a dozen different manufacturers. At the present time we have two viable suppliers (Element6 and II-VI Ltd.). Both have the capability to produce tracker quality substrates and have demonstrated the capacity to produce several hundred $\mathrm{cm}^{2}$ per year of sensor material. Figure 3 shows the size of the various diamond systems that have been installed over the last 10 years. The current capacity, and the potential manufacturers have to increase that capacity, should be sufficient to build a two or three tracking layers at radii up to $10 \mathrm{~cm}$ in both the multi-purpose LHC experiments prior to the ultimate luminosity running in the next decade.

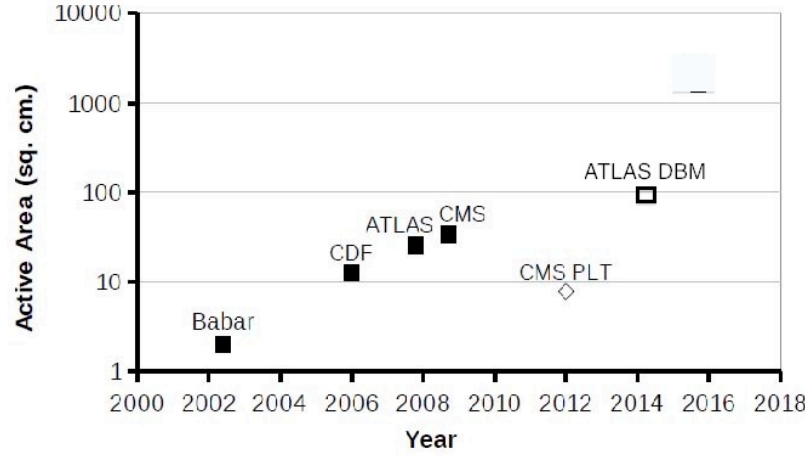

Figure 3: Active area of various diamond detector systems that have come in to operation over the last ten years. The open symbols show tracking devices that are currently under construction.

Diamond sensors have proven highly reliable replacements for silicon in every HEP application they've been used in over the past 10 years. In each case they've allowed measurements that were un-expected when they were first proposed. We expect that they will continue to revolutionize the way we do tracking in high occupancy, high radiation environments at the high luminosity LHC and beyond.

[1] W. Adam et al., Nucl. Inst. Meth. A565 (2006) p278.

[2] M. Mathes et al., JInst 3 (2008) P12002.

[3] W. Adam et al., Nucl. Inst. Meth. A514 (2003) p79. 
April 16, 2013

\section{A Differential Time-of-Flight Technique for Collider Detectors

\author{
Henry J. Frisch \\ Enrico Fermi Institute; University of Chicago
}

For the LAPPD Collaboration

The growth of capabilities of large particle detectors at high-energy collider accelerators is truly remarkable- the contrast in capabilities between ATLAS and CMS, and UA1, UA2, or Mark-I, for example, is a testament to the technical and managerial strengths of the field of HEP. However, while the development of the measurement of particle 3-vectors has been the subject of a large investment, the measurement of 4 -vectors has languished. These big detectors are precision tools, and are capable of reconstructing invariant masses at great precision from combinations of tracks. The particle mass for a given track is the only information other than spin that remains to be measured. To get our money's worth we should extract all the information available from each and every collision.

In addition, for hadron colliders, as the achievable luminosity rises the problem of the association of particles with the correct one of many vertices becomes severe, and can limit the precision as well as lead to errors in track and event reconstruction. Associating photons with vertices, as one might want to in events with high $P_{T}$ photons in possible Higgs, KK, or gauge-mediated SUSY signatures, has been even more difficult. Psec-resolution timing adds another, strong, constraint on the vertex position for both charged particles and photons.
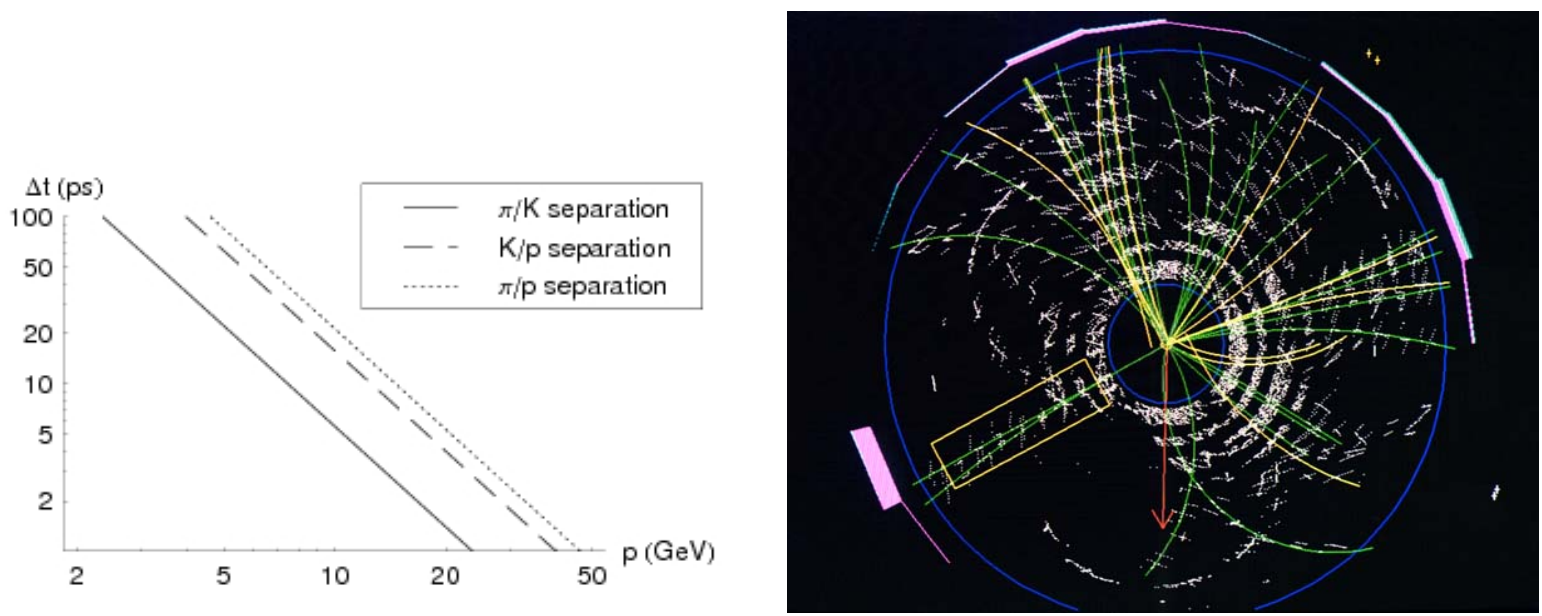

Figure 1: Left: The delay in arrival in psec of pions, kaons, and protons compared to the photon arrival time, vs the log of the transverse momentum. Right: A top event from CDF showing the tracks and photons arriving at the outer radius of the solenoid coil. The differential timing proposal for a collider detector would implement fast timing 'tiles', with the local (space and time) calibration being given by the difference in arrival times of charged particles and the nearby photons or electrons used for calibration for a given vertex.

In addition to precision reconstruction and parton flavor-tracking, precise timing allows searches for new heavy or metastable particles that arise in the many models of new physics. 
The recent work on psec or sub-psec timing opens the possibility of large-area TOF systems for colliders $[1,2,3,4]$. A resolution of 1 psec gives $1-\sigma$ separation of pions and kaons at $P_{T}=21 \mathrm{GeV}$, as shown in Figure 1 .

With large signals, such as one would expect from a Cherenkov radiator faceplate traversed by a charged particle at a collider, the LAPPD Collaboration has achieved a resolution of $\leq 5 \mathrm{psec}$ on the difference of pulse arrival times at opposite ends of an 8" 'frugal' glass anode [5], driven by a pair of MCP plates with $20 \mu$ pores [6]. Improvements in pulse fitting and noise reduction in the present setup should reduce this to a few psec or less.

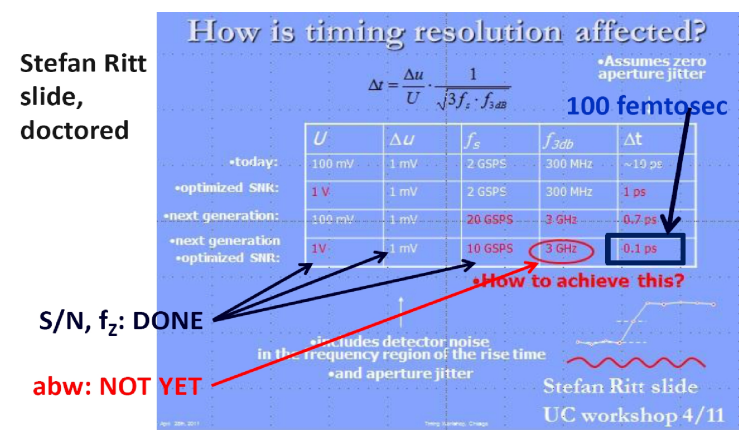

Figure 2: A simple but intuitive parameterization by Ritt [7] of the time-of-flight precision versus the analog bandwidth, signal-to-noise, and waveform sampling rate.

Figure 2 shows a simple but well-motivated parameterization by Ritt [7] of the time-offlight precision versus the analog bandwidth, signal-to-noise, and waveform sampling rate. If one holds the number of samples constant, this 'rule-of-thumb' translates into the resolution depending linearly on the risetime and inversely on the S/N-ratio. Risetimes of $60 \mathrm{psec}$ have been achieved [8], and signal-to-noise ratios appreciably greater than 100 have been measured with sampling rates exceeding $10 \mathrm{GS} / \mathrm{sec}$ [9]. Looking at the bottom row of the table in Figure 2, the extrapolated timing resolution is significantly less than 1 psec [10]

Beyond this, we have now a good understanding of the determining factors for better time resolution [11, 12]. Higher-bandwidth anodes [13], faster rise-times (i.e. higher bandwidths) from smaller pores [8], and customized secondary-emitting structures [14] have the potential of moving solidly into the regime of sub-picosecond TOF resolution.

However, even if this can be achieved locally, it will be very difficult to build a large TOF system that has a precision of 1 psec or better over long times and large detector subsystems. The proposal here is 'differential TOF', in which the time-of-arrival of particles is measured relative to the first particles from the same vertex to arrive nearby at the TOF counters. Thus the first particles should be photons and electrons, followed by muons, pions, kaons, protons, etc., depending on momentum. The differences in timing are small, as shown in Figure 1; the goal is to measure them directly. This reduces both the requirements on stability vs time of the clock reference and of the cross-calibration over the detector, as the former are short, and the latter are local and involve only a local, shared, clock.

The first step in testing the concept will be in a test beam, with several stations of LAPPD or equivalent counters in close proximity. The next step, presumably, would be to test several LAPPD tiles in either a test beam with a target to measure TOF with multiple particles from a common vertex, or as a test in an active experiment [15].

I thank all my LAPPD collaborators for their essential contributions on which the 
idea of differential TOF at colliders rests.

\section{References}

[1] Visions of Experimental Particle Physics - Where Are We Going? (Invited Summary Talk, Aspen Winter Conference; January 26, 2003).

[2] T. Credo (IMSA), H. Frisch, H. Sanders, R. Schroll, and F. Tang; Picosecond Timeof-Flight Measurement for Colliders Using Cherenkov Light; Proceedings of the IEEE, Rome, Italy, Oct. 2004; Nuclear Science Symposium Conference Record, 2004 IEEE, Volume: 1 Date: 16-22 Oct. 2004.

[3] K. Inami, N. Kishimoto, Y. Enari, M. Nagamine, and T. Ohshima; Nucl. Instr. Meth. A560, p.303, 2006

[4] The original LAPPD institutions include ANL, Arradiance Inc., the Univ. of Chicago, Fermilab, the Univ. of Hawaii, Muons,Inc, SLAC, SSL/UCB, and Synkera Corporation. More detail can be found at http://psec.uchicago.edu/.

[5] RF strip-line anodes for Psec large-area MCP-based photodetectors; H. Grabas, R. Obaid, E. Oberla, H. Frisch, J.-F. Genat, R. Northrop, F.Tang, D. McGinnis, B. Adams, M. Wetstein; Nucl. Instr. Meth, Feb., 2013

[6] The glass capillary substrates are produced by Incom Inc. Charlton Mass. See http://www.incomusa.com/.

[7] S. Ritt, in The Factors that Limit Time Resolution in Photodetectors; Workshop, Univ. of Chicago, Chicago, IL; 28-29 April 2011. See http://psec.uchicago.edu/workshops/ Note that of the values needed of the four parameters to achieve a time resolution of $100 \mathrm{fsec}$ (the bottom row of the table of extrapolations), we have achieved or exceeded three: sampling rate, noise, and signal size. Only the analog bandwidth falls short at present.

[8] J. Milnes and J. Howorth (Photek Ltd.), "Advances in Time Response Characteristics of Micro-channel Plate PMT Detectors". See http://www.photek.com/support/technical_papers.htm (website says: 'Ref. not yet available').

[9] E. Oberla, A Fast Waveform-Digitizing ASIC-based DAQ for a Position \& Time Sensing Large-Area Photo-Detector System; Photodet2012, LAL Orsay, France; June, 2012

[10] At the predicted level of 100 fsec many new system factors will come into play; the point is that the intrinsic resolution of the detector itself will be very small, and substantial effort should be devoted to the other limiting factors.

[11] J.-F. Genat, G. S. Varner, F. Tang, H. Frisch; Signal Processing for Pico-second Resolution Timing Measurements, Nucl. Instr. Meth. A607, p387, Oct. 2009; e-Print: arXiv:0810.5590 
[12] For a discussion of the factors that determine time and space resolution in MCPbased detectors, see the talks at: The Factors that Limit Time Resolution in Photodetectors; Workshop, Univ. of Chicago, Chicago, IL; 28-29 April 2011. See http://psec.uchicago.edu/workshops/

[13] L. Sadwick, R. J. Hwu, H. Frisch; A High Bandwidth LAPPD Anode; 2012 DOE STTR.

[14] J.W. Elam, J. A. Libera, M.J. Pellin, and P.C. Stair, "Spatially Controlled Atomic Layer Deposition in Porous Materials", Applied Physics Lett., 200791 (24)

[15] A short test measuring TOF of converted photons to reconstruct pizero decays at KOTO at JPARC would be very interesting, as the photons would give large signals and hence large $\mathrm{S} / \mathrm{N}$. 


\section{Future Developments in Gigasample-per-second Waveform Sampling Application Specific Integrated Circuits}

Names: Eric Oberla, Kurtis Nishimura

Time Frame: Intermediate term

Physics Justification: Modern high energy physics and astrophysics experiments are increasingly making use of waveform sampling (WFS) technologies. In contrast to previous generations of readout electronics where one TDC and one ADC value might be stored per channel, recording full waveforms allows experimenters to perform real-time or offline digital signal processing, pulse shape analysis, cross-talk correction, and precision timing extraction. While many experiments can utilize commercial digitizers (typically flash ADCs), such components may not viable if the experiment calls for some combination of multi-GSa/s sampling rates, low-power, or very high channel density.

Custom WFS ASICs fill this void in the available portfolio of front-end readout electronics. Such ASICs provide a novel approach to meeting these experimental challenges. They are capable of analog sampling at rates of several $\mathrm{GSa} / \mathrm{s}$ to over $10 \mathrm{GSa} / \mathrm{s}$ at power levels of tens of $\mathrm{mW}$ per channel during sampling. The input analog bandwidths of these ASIC designs span a range of $\sim 100 \mathrm{MHz}$ to over $1 \mathrm{GHz}$.

Such electronics are already in use at the cosmic [1-3], intensity [4-5], and instrumentation [6] frontiers. For example, this technology has served as the key enabling component for the ANITA experiment's search for ultra-high energy cosmogenic neutrinos, due to its tightly constrained power budget and its need for timing resolutions at the $\sim 10 \mathrm{ps}$ level [7].

The cost of WFS ASICs is also relatively low, from tens or hundreds of dollars per channel in prototype quantities, down to a few dollars per channel when produced in large quantities.

\section{Technical limitations:}

The current generation of WFS ASICs have several technical challenges. Two of the primary design challenges are presented:

1) Buffer Depth: These waveform samplers are effectively 'analog down-conversion' devices, in which a waveform is recorded at a rate of several-GSa/s and data is read off-chip at a slower rate, typically 10-100 MHz. Several designs incorporate an on-chip ADC that adds an intermediate latency step. Therefore, the 'event' waveform sample depth is limited by the on-chip buffering architecture.

Many of these custom WFS ASICs are limited to short buffer depths, typically less than 1 us, and in some cases as short as $25 \mathrm{~ns}$. These ASICs are usually operated in a triggered mode, in which a system trigger initiates conversion and data readout. This experimental trigger latency sets the minimum required buffer depth of the front-end WFS ASIC.

WFS ASIC designs with very deep buffers, up to tens of microseconds, are currently being investigated. While this promises the possibility of accommodating long trigger latencies and effectively dead time-less operation below a given event rate, this gain may be offset by an increased nonlinearity, more calibration constants, higher power, and increased die size.

2) Calibrations: The typical technique of using delay lines to generate the fast effective sampling rates imposes a large number of timing calibration constants on the data from these ASICs. These calibrations must performed be if optimal timing resolution is to be obtained. While techniques exist to deal with these issues, they impose a significant burden on the analysis of such data. 
Other calibrations, such as nonlinearity and pedestal corrections, are also required for optimal ASIC performance. Though these calibrations are relatively straightforward, the number of calibration constants will scale with the increased ASIC analog buffer.

Technical Capabilities: A few programs in the US are actively involved in designing and characterizing fast WFS ASICs. Most development is centered around expertise at LBNL / UC Irvine, the University of Chicago, and the University of Hawaii. Fabrication of ASICs is typically brokered through MOSIS, either through multi-project wafers for prototype runs, or dedicated runs for larger quantities.

Key R\&D Directions: Continued work is required to fully characterize the tradeoffs that are available in terms of sampling rates, buffer depths, power consumption, and other features. The first long buffer depth (many $\mu \mathrm{s}$ ) WFS ASICs are being developed for experiments that will begin within the next few years.

There is an ongoing effort targeted at developing calibration and characterization techniques for these ASICs. This work is particularly important, as it dramatically reduces a significant barrier to entry for those interested in adopting fast waveform sampling technology for their experiments.

A further evolutionary step would be to build in features to these ASICs that can perform calibration at the hardware level, thus allowing downstream systems to deal with a significantly simplified data stream. This work is just beginning, and if successful, could dramatically widen the potential applications for these ASICs.

Additional developers of WFS ASICs, support electronics, and calibration systems that can be implemented in hardware and software, would be particularly helpful to ensure that these developments can be fully explored and realized in a timely manner.

\section{Bibliography:}

[1] E. Delagnes, et al. "SAM: A new GHz sampling ASIC for the H.E.S.S.-II Front-End Electronics", Nucl. Instr. Meth A 567 (2006) 21-26.

[2] S.A. Kleinfelder, "Multi-GHz Waveform Sampling and Digitization with Real-Time Pattern-Matching Trigger Generation”, SORMA 2012 Proceedings (2012).

[3] K. Nishimura, et al, "A low-resolution, GSa/s streaming digitizer for a correlation-based trigger system”, arXiv:1203.4178v1 (19 March 2012)

[4] G.S. Varner, et al, "The Large Analog Bandwidth Recorder and Digitizer With Ordered Readout (LABRADOR) ASIC", Nucl. Instr. Meth A 583 (2007) 447-460.

[5] S. Ritt, "The DRS Chip: Cheap Waveform Digitization in the GHz Range”, Nucl. Instr. Meth. A 518 (2004) 470-471.

[6] E. Oberla, et al, "A $15 \mathrm{GSa} / \mathrm{s}, 1.5 \mathrm{GHz}$ Bandwidth Waveform Digitizing ASIC”, submitted to Nucl. Instr. Meth A (2013). (pdf available: http://psec.uchicago.edu/library/doclib/documents/218/sendit) [7] P. Gorham, et al "The Antarctic Impulsive Transient Antenna Ultra-high Neutrino Detector Design, Performance, and Sensitivity for 2006-2007 Balloon Flight”, Astropart.Phys. 32 (2009) 10-41. 


\title{
Opportunities for HEP Technology Evaluation in Smaller-Scale NP Experiments
}

\author{
P. Nadel-Turonski (JLAB) and G. Varner (University of Hawaii)
}

Needs: The relatively small number of new HEP experiments and their very long development and operation timescales mean that few opportunities exist to develop technologies needed for future experiments. At the same time, oversubscription of the limited number of test beamlines and the need for careful characterization of detector events, in ways best done in a more complete experiment, are increasingly important.

Opportunity: Experiments in NP have similar needs in terms of detector technologies but tend to be smaller in size and materialize more frequently and on a shorter time scales. This opens creates a potential for synergies. While the initial stages of detector $R \& D$ include work in the lab and with test beams, the ultimate test is to use the detectors in a real experiment. To first use detectors planned for a large-scale HEP experiment in a smaller NP experiment would demonstrate the viability of the concept, allow for incorporating lessons learned before final construction, give students working on the project a chance to see actual data taken as a result of their efforts, and create a very tangible project milestone. The benefits for the NP experiment could also be significant, as it could make it easier for experiments to select new technologies instead of more conventional systems, benefiting from the R\&D that had been undertaken. One could also imagine that there would be mutual benefits where funding for the NP experiment could be combined with funding for HEP prototype construction, allowing a more efficient global allocation of DOE resources. While such a close collaboration between HEP and NP may not work for all projects, it would definitely be worthwhile to take advantage of emerging opportunities.

Time Frame: Current, Intermediate, and Long

A Concrete Example: A concrete example of such cooperation between HEP and NP has been the Time Of Propagation detector for Belle II. This advanced particle identification detector has been prototyped as part of the upgrade of the Belle detector. The LEPS collaboration at SPring-8 is also interested in this technology as part of the LEPS-2 upgrade. To gain experience with the technology involved, a set of collaborative beam tests have been undertaken together at SPring8, where the prototype devices used well reconstructed positrons in the LEPS spectrometer as a test beam source. Excellent test results have been obtained, which are essential for demonstrating that the detector is ready for production for Belle II, and a variant of the detector will be constructed to improve the particle identification of the LEPS-2 detector.

Resource Requirement: Some mechanism for fostering a dialog between HEP and NP experimenters on the possible opportunities for such collaboration. 


\title{
Applications of Laser Interferometry in Fundamental Physics Experiments
}

\begin{abstract}
Laser interferometry provides a new and uniquely powerful tool to probe certain aspects of physics, such as exquisitely precise differential position measurements, and controlled, coherent macroscopic quantum states of electromagnetic fields. Although developed primarily for gravitational wave studies, they can be extended with additional R\&D to study the fundamental quantum nature of geometry, or interactions of electromagnetic fields with new types of fields, such as axion-like particles.
\end{abstract}

"Particle Physics" and "High Energy Physics" have outgrown their names. The fundamental nature of the physical world depends on relationships between matter, energy, space and time; some of the most interesting aspects of this relationship depend on spatially extended states (that is, waves and not particles, as in neutrino oscillations), while others, most notably cosmic Dark Energy, also depend on physics at the very lowest energy densities.

The advanced technology of laser interferometry developed for gravitational wave research in recent years provides a new set of experimental tools that can be adapted for experiments in fundamental physics. The capabilities include the ability to measure position with extreme (attometer-scale) precision; to prepare and maintain macroscopic (>meter-scale) systems in coherent quantum-mechanical configuration states; and to create large-scale, coherent states of electromagnetic fields, to study their possible interactions with new forms of matter and energy, such as axion-like particles.

A state of light in a laser cavity shapes the modes of the electromagnetic fields in a particular way: they are confined in space by boundary conditions imposed by mirrors, and their states are entangled with the positions of those macroscopic bodies. The particles or "photons" in these systems are extended in more than one spatial dimension, with a shape that can be controlled. Their quantum state can also be squeezed in phase space. These capabilities provide unique tools to study new physics.

As one example, they can be used to constrain quantum effects of space-time arising at the Planck scale, $c t_{P} \equiv \sqrt{\hbar G / c^{3}}=1.616 \times 10^{-35} \mathrm{~m}$. In standard quantum field theory, space-time is self-consistently assumed to behave classically. However, new quantum effects of geometry originating at the Planck scale - from geometrical degrees of freedom not included in standard field theory - could have effects on macroscopic scales.

The possibility of new quantum-geometrical degrees of freedom is suggested from several theoretical directions. Quantum physics is experimentally proven to violate the principle of locality on which classical spacetime is based. Gravitational theory suggests that quantum states of space-time systems have much less information than predicted by quantum field theory, and suggests that space-time and gravity are approximate statistical behaviors of a quantum system with a holographic information content. [1, 2]
A typical uncertainty in wave mechanics, if information about transverse position is transmitted nonlocally with a bandwidth limit, is the scale familiar from diffractionlimited imaging: the geometric mean of inverse bandwidth and apparatus size. For separations on a laboratory scale, a Planck scale frequency limit leads to a transverse uncertainty in position on the order of attometers. Quantum geometry may be detectable as a new source of quantum-geometrical noise in an interferometer that coherently measures the positions of massive bodies in two directions over a macroscopic volume.[3-5] The Fermilab Holometer experiment (E-990), funded largely through an Early Career Award to Aaron Chou at Fermilab, is designed to detect or rule out quantum-geometrical noise with these properties. [6]

Much of the Holometer technology has been developed by LIGO and other projects to measure displacements due to gravitational radiation. The quantum-geometrical measurement however calls for application of the technology in a new experimental design, with new challenges. If holographic noise is found, a significantly expanded experimental program can be pursued to obtain high precision results and map out the spatiotemporal properties of quantum geometry.

Similarly, spatially extended laser cavities can greatly amplify, via quantum coherence, the interaction of electromagnetic fields with new low-mass fields, such as axion-like particles. In this case, laser cavities may be employed together with large magnets to explore mediating interactions with the new particles.

Profound mysteries such as Dark Energy probably involve new physics that is both non-local and low-energy in character - a departure from the field theory framework that will require new kinds of techniques to study. Laser interferometry has already started to show its unique value and should be developed further by the HEP community.

[1] T. Jacobson, Phys. Rev. Lett. 75, 1260 (1995)

[2] E. P. Verlinde, JHEP 1104, 029 (2011)

[3] C. Hogan, arXiv:1208.3703 [quant-ph].

[4] C. J. Hogan, arXiv:1204.5948 [gr-qc].

[5] C. J. Hogan, Phys. Rev. D 85, 064007 (2012)

[6] http://holometer.fnal.gov 


\section{Large arrays of SiPMs for HEP and industry}

The Instrumentation Frontier (IF) working group has identified and opportunity for the US to lead the development of large arrays of Si photo-multipliers (SiPM) for high energy physics (HEP) experiments with possible future applications in everyday imaging.

Private companies are vigorously pursuing the development of SiPM technology for the medical industry, night vision and lidar. The resources available to this sector are much larger than those available to the basic research community. However, these are focused on addressing the very specific needs of a "product" and are constrained by market pressures. The HEP community needs to be agile taking advantages of these developments, adapting them to the science needs and building on them for the next generation of experiments. These science-driven developments are likely to contribute in the longer term to new applications for everyday life.

Possible future applications for large arrays of SiPM in the cosmic frontier include gamma ray telescopes[1], air shower fluorescence telescopes for [2][3], optical astronomy[4] and direct DM detection on TPCs[5]. The intensity frontier is also considering large arrays of SiPM for the next generation experiments [6]. The effort of several relatively small groups in the US and abroad has produced steady progress towards these applications. However, a coordinated program to advance this technology for HEP is required to ensure its availability for the next generation of experiments. The main areas in need of R\&D are the development of low power readout systems for large arrays, fully digital SiPM and high rate DAQ. A strong program of SiPM R\&D in HEP, would also push the manufacturers of these devices to address some features that are important for these experiments (detection efficiency, afterpulsing, crosstalk, etc).

The US has currently a few companies competing in the manufacturing of these photodetectors [7]. Several university groups are doing research on these sensors, and our national labs have been investing in R\&D for these sensors. For this reason we believe that there is enough critical mass in the US to be a major contributor to this technology.

\section{References}

[1] Talk by Weinstein@ANL and white paper

[2] Talk by Privitera@ANL)

[3] Extreme Imaging Workshop at University of Chicago (2013)

[4] Talk by J.Estrada at the Cosmic Frontier meeting at SLAC

[5] arXiv:1206.6199

[6] arXiv:1305.7245

[12] http://www.excelitas.com; http://voxtel-inc.com/; http://www.amplificationtechnologies.com/products.htm; http://www.rmdinc.com/; http://www.lightspintech.com/ 


\title{
Snowmass 2013 White Paper
}

\section{Ultra-Low Index and Rad-Hard Total Internal Reflection Claddings for High Numerical Aperture Scintillating, Waveshifting, Cerenkov, or Clear Optical Fibers, Capillaries, and Plates}

\author{
C. Sanzeni, G. Jennings, D.R. Winn* \\ Fairfield University \\ Fairfield, CT 06880 USA
}

\author{
*Corresponding Author \\ winn@fairfield.edu \\ $1+203.984 .3993$
}

\begin{abstract}
Novel low index $(\mathrm{n} \leq 1.3) /$ high light trapping cladding films consisting of nanoporous alumina (sapphire), as formed by controlled anodization of aluminum, are described. These films are mechanically hard, intrinsically very rad-hard, and have an index of refraction $\mathrm{n}$ sufficiently small to triple the light capture of waveshifting or scintillating fibers and transparent plastic, glass or quartz core fibers. The low indices enable light-piping of water Cerenkov light. Applications are in Energy, Intensity, and Cosmic/Astroparticle detectors.
\end{abstract}

Introduction: Optical fiber and light-transmitting Total Internal Reflection (TIR) technology is frequently used in High Energy, Astroparticle, and Nuclear Physics research. Uses include organic scintillating (SciFi) and wavelength shifting (WLS) fibers, quartz Cerenkov fibers, and clear fibers, used to transport analog signals. They are used in calorimetry, rpreradiators and tracking, often in high magnetic fields, and others, including in telescope spectroscopy image planes. Generally, except for quartz core/doped quartz claddingsfibers, they have low radiation hardness, and a modest numerical aperture (N.A.), especially doped quartz cladded fibers(NA 0.2). We propose to extend radiation hardness to ultra-low index (high $\mathrm{NA}>0.8$ ) claddings for superior light capture, increased by as much as a factor of 3 in transmitted light fractions. This cladding technology is easily applied to scintillating, WLS or Cerenkov plates, fibers or capillaries.

Low Index Rad-Hard Fiber Claddings: An extensive review of fibers used in HEP experimentation is given by H.Leutz ${ }^{1}$, CERN. Typical plastic fiber performance is shown in the figures below.
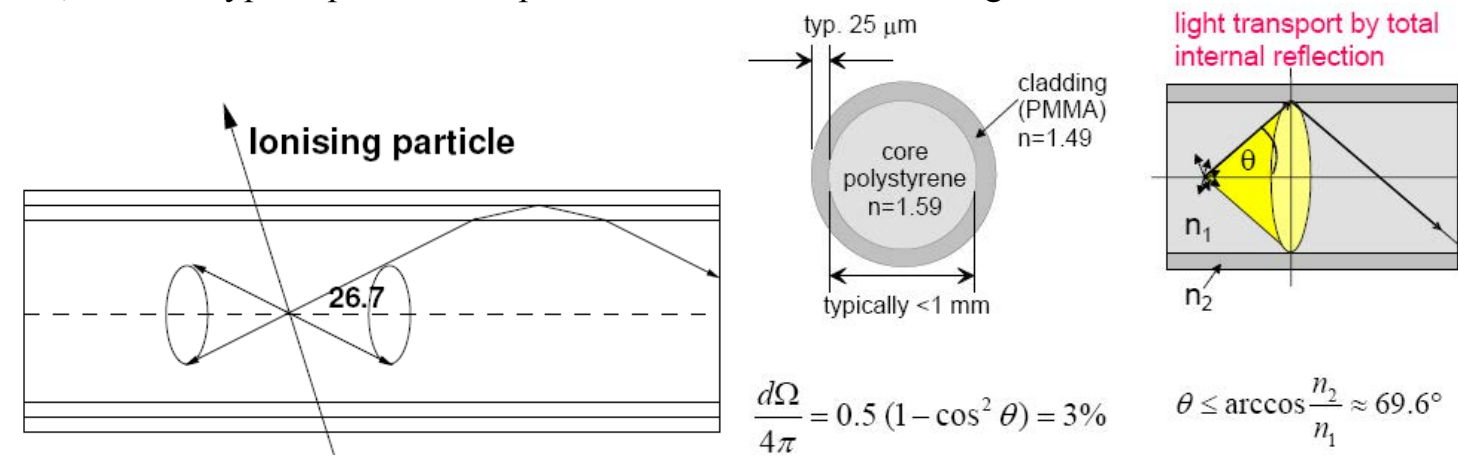

Fig. 1: Cartoons of light trapping in a typical plastic WLS fiber. The angle $\theta$ is the trapped cone angle.

The fraction of light piped to each end of a fiber is shown in the cartoon of Fig.1. The fraction $\mathrm{f}$ piped is given by the cladding $\mathrm{n}_{2}$ and the core $\mathrm{n}_{1}$, the critical angle $\alpha$ and the Numerical Aperture NA by:

$$
\begin{aligned}
& \mathrm{f}=1 / 2\left(1-\mathrm{n}_{2} / \mathrm{n}_{1}\right) . \\
& \sin \alpha=\mathrm{n}_{2} / \mathrm{n}_{1} \\
& \mathrm{NA}=\left(\mathrm{n}_{1}{ }^{2}-\mathrm{n}_{2}^{2}\right)^{1 / 2}=\sin \theta
\end{aligned}
$$


Typical fractions per end are $\mathrm{f} \sim 3 \%$ for commercial plastic fibers. If the cladding could be made to have an index of 1.3, the fraction trapped would rise to $\sim 9 \%$, tripling the trapped light fraction. A cladding index of 1.4 would double the fraction of trapped light, to $\sim 6 \%$, as compared to the example with a polystyrene core and a PMMA cladding as shown above. The number of reflections in terms of the diameter core $\mathrm{d}$ length of fiber $\mathrm{L}$ critical angle $\alpha$ by: $\mathrm{N}=\cot \alpha \mathrm{L} / \mathrm{d}$. For small diameter fibers, the imperfections in the walls dominate. The reflection loss length $L_{R}$ over which the injected intensity is reduced by $1 / \mathrm{e}$ from reflection imperfection losses $0<\mathrm{q}<1$ (i.e $\mathrm{q}$ is the effective the reflectivity back into the trapped region) is given by: $L_{R} \sim 1.5 /(1-q)\left(n_{1} / N A\right) d$. For many scintillators and claddings this will dominate over scintillation light self-absorption for small diameter fibers. In general, $\mathrm{L}$, the $1 / \mathrm{e}$ total attenuation length is given by: $\mathrm{L}^{-1}=\mathrm{L}_{\mathrm{A}}^{-1}+\mathrm{L}_{\mathrm{R}}^{-1}+\mathrm{L}_{\mathrm{s}}^{-1}$, where $\mathrm{L}_{\mathrm{s}}, \mathrm{L}_{\mathrm{A}}$ are the scattering and absorption lengths. The cladding must be $>4$ wavelengths thick.

\section{Novel Low Index of Refraction Radiation-Hard Hardcoating Technology for Optical Material Total Internal Reflection Claddings.}

To obtain a very low index cladding film, we have developed a novel method to anodize completely metal films applied to insulators, so that no metal remains, except for an arbitrarily small connection line at an edge of the work piece. The controlled anodization of metals under proper conditions results in a hard metal-oxide film with an ordered nanoporosity, up to $\sim 70 \%$ porous in hexagonal close packing, with the pore diameters well-less than $100 \mathrm{~nm}<\lambda_{\text {light, }}$, and the pore lengths up to $\sim 100$ times longer than the diameters (i.e. porous film thickness $\sim \mu \mathrm{m}$ 's $\sim$ several optical $\lambda$ ). The resulting index $\mathrm{n}$ is dominated by the index of the air in the pores, and thus is low (see below).

The formation of arrays of high aspect ratio nanopores during the anodic oxidation of ultra-pure aluminium is a well-known phenomenon. The occurrence of a near-periodic pore arrangement in porous anodic alumina was first reported by Masuda et al. in the early 1990's (see refs ${ }^{2}$ and loc. cite). The areal density, size, and regularity of these pores is controllable over a remarkably wide range with the electrolyte chemistry, temperature, current density, and the electric field during the anodization. Recent work is shown in Figures 3. Films of $\mathrm{Al} \sim 3 \mu \mathrm{m}$ thick resulted in uniform porous structures, the pore diameter being in the range of 10-30 nm and their density of the order of $8-9 \times 10^{10}$ pores $\mathrm{cm}^{-2}$. Pore widening with phosphoric $\operatorname{acid}^{3}$ can easily increase the open area to above $70 \% \mathbf{i}$.

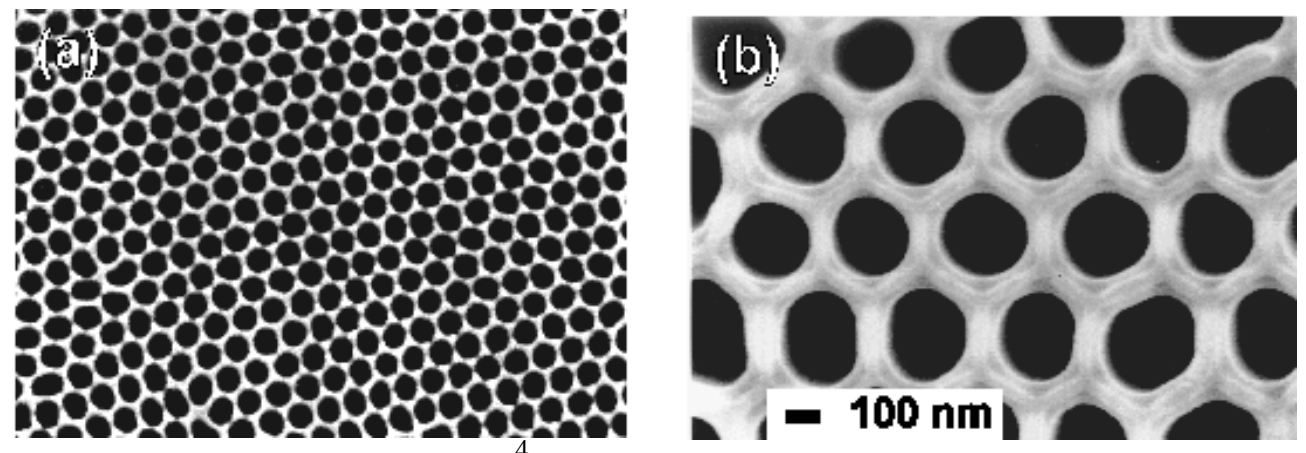

Figure 3a, b: Anodic alumina nanoporous films ${ }^{4}$. Left $-30 \mathrm{~nm}$ pores. Right $\sim 130 \mathrm{~nm}$ pores.

\footnotetext{
i As an aside, filling the high aspect pores with metal or other conductor forms a "z-axis" connector, an anisotropic conducting film or sheet which has very low resistance through the film(z-direction) and very high resistance in the $\mathrm{x}-\mathrm{y}$ plane of the film. Thus 2 planar VLSI circuits can be connected by sandwiching such a film between 2 silicon substates, without top/bottom registration, if the pores are smaller and more dense than the circuit features.
} 

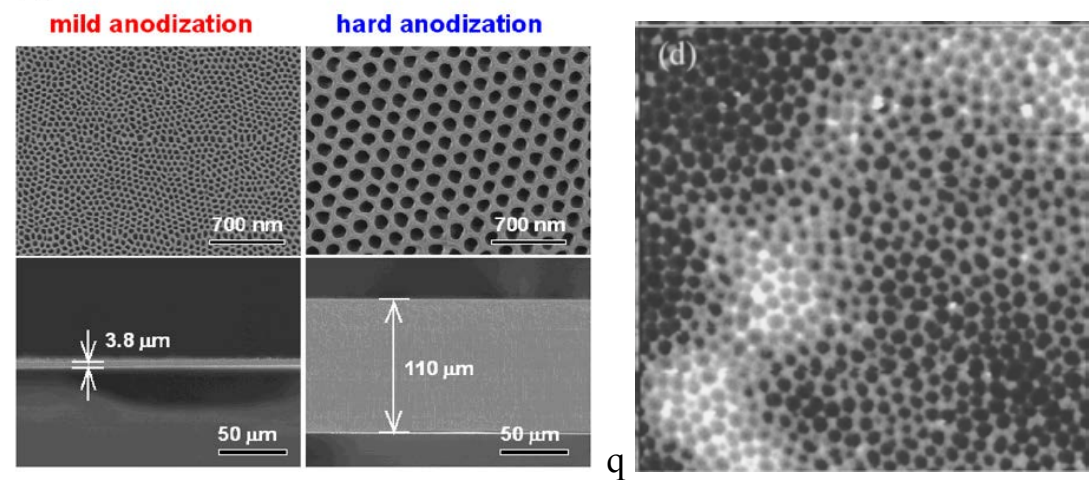

Figure 3c(Left): Ultra-thin porous anodic alumina films with 2 anodization protocols, with self-ordered cylindrical vertical pores on a silicon substrate ${ }^{5}$ Note the film thicknesses. The pores sizes are as compared with the $700 \mathrm{~nm}$ fiducials. Either film can be pore-widened with post anodization isotropic etch. Fig. 3d(Right): A $2 \mu \mathrm{m}$ x $2 \mu \mathrm{m}$ square SEM field of anodic alumina. The pores are $\sim 50 \mathrm{~nm},<$ optical wavelengths.

Because the pores are far smaller than visible wavelengths, the resultant heterogenous films can be considered uniform for the optical properties of visible light, and have an index of refraction for heterogeneous media given by several possible formulae ${ }^{6}$ which bracket the possible extremes - (a) Parallel: $\mathrm{n} \sim \mathrm{fn}_{\mathrm{p}}+(1-\mathrm{f}) \mathrm{n}_{\mathrm{o}}$; (b) Series: $1 / \mathrm{n} \sim \mathrm{f} / \mathrm{n}_{\mathrm{p}}+(1-\mathrm{f}) / \mathrm{n}_{\mathrm{o}}$; or (c) Drude: $\mathrm{n}^{2} \sim \mathrm{fn}^{2} \mathrm{p}+(1-\mathrm{f}) \mathrm{n}^{2}{ }_{\mathrm{o}}$ where $\mathrm{f}$ is the porous fraction of the film, $\mathrm{n}_{\mathrm{p}}$ is the pore index, equal to that of air $\left(\right.$ i.e. $\left.\mathrm{n}_{\mathrm{p}}=1\right)$ unless filled, and $\mathrm{n}_{\mathrm{o}}$ is the oxide matrix index. For the form of Aluminum Oxide here (boehmite, $\gamma$-alumina), for example, $\mathrm{n}_{\mathrm{o}} \sim 1.75$, and so for $\mathrm{f}=65 \%$ porosity, easily achieved, the index of the film is bracketed by $\mathrm{n}$ in the ranges $1.26 / 1.18 / 1.3$, respectively as calculated for the 3 formulae above, low enough to capture significantly more light in fibers with such a cladding. NOTE: For $\mathrm{n}<1.33$, such a material could pipe light from a water core $\mathrm{n}=1.33$ (if the pores were sealed with an obliquely applied film, as commonly used in anodic products, or coated with an ALD hydrophobic film), a significant additional tool for large neutrino \& proton decay detectors. As discussed above, this low cladding increases the trapping fraction significantly and enables far better detection even with the thinnest fibers ${ }^{7}$. At cladding $n=1.3$ with a core index of 1.6, the $N A=0.93$, and $9 \%$ of the light is transmitted, a factor of 3 over the best plastic fibers. For quartz cores $(n=1.54), 8 \%$ of the light is transmitted. For a water-core light guide, with a cladding of $1.26,2.7 \%$ of light is transmitted.

Normally a metal film-on-insulator, or any metal, when immersed in an anodization bath and connected to an anode power supply anodizes to form an insulator (metal oxide) layer at the surfaces in contact with the electrolyte. The anodized layer, even if porous, then prevents further anodization from occurring, unless new metal is exposed to the electrolyte, and a conducting connection is supplied to the remaining metal. Even in the case of porous anodization, a boundary layer of metal must be left; as the metal anodizes, the ability to conduct current is reduced to near zero, leaving metal surrounded by anodized metal. Using the standard anodizing technique, it would be impossible to anodize a metal film on an insulator, such as a quartz fiber or plate completely - i.e. leaving no unanodized metal. For example, an aluminum film on an insulator would anodize until either islands of metal remained, or a continuous film of metal remained on the insulator. Even if a through-insulator connection to the back side of the metal were provided, isolated islands of unanodized metal would result.

However, we have demonstrated a simple method to anodize a metal film on an insulator or thin plate of aluminum completely. This method can also be used to control the currents on the sample surface by limiting the area of aluminum presented to the electrolyte in the anodization process. In principle, the technique can be implemented in several ways. The method we have demonstrated in our lab is shown schematically in Fig.4 below: 


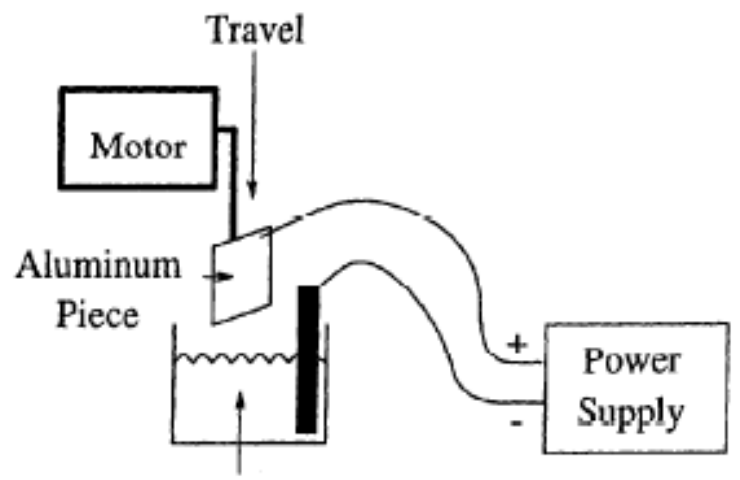

Anodization bath

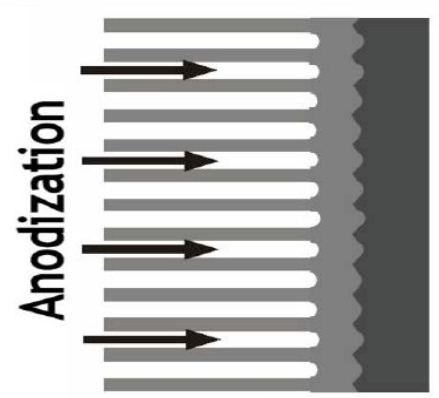

Figure 4: Schematic view of the edge anodization technique where aluminum pieces are controllably submerged into the anodization bath, in our experiment by a feed-back driven stepper motor. The cathode connection to the electrolyte (anodization bath) is represented by the thick electrode, usually a lead or carbon sheet.

The thin aluminum piece (i.e foils) or aluminum film on an insulator, such as a transparent plastic scintillator or WLS fiber core, is mounted on a motorized stage which allows the sample to be lowered into the anodization bath at a controlled velocity. The key idea is that the section of the piece just above the anodization bath acts as the anode terminal connection, an edge connection equal at all parts of the cross-section through the thickness of the aluminum piece. As the piece is "adiabatically" lowered into the bath, in effect the anode terminal is presented equally to all parts of the unanodized metal in the bath, unlike the situation if the anodization starts on a fully immersed film, where metal not in direct contact with the electrolyte becomes insulated by the barrier oxide formed on the metal surrounding it. Only the thin stripe of aluminum which is just below the bath surface is unanodized, but connected to the same potential. The entire metal film is thus able to be anodized as the metal is immersed methodically into the electrolyte. We call this technique Edge Anodization or EA.

The key technical and scientific challenge is to provide control of the insertion motion to anodize the film with uniform quality. This motion can be controlled with feedback on the anodization current or current density. There are several constraints on the sample velocity. If the velocity is set too high, then the cell current/voltage (I/V) characteristic will be dominated by the current drawn as the virgin aluminum surface develops its oxide barrier. In the limiting case of high velocity, it is the same as anodizing the entire surface at once. If the velocity is too low, then the quality of the aluminum surface and the electrolyte/ aluminum interface will determine the quality of the anodized film. Extremely low velocities and unclean surfaces could lead to unanodized areas of the aluminum surface.

Anodized aluminum and the formation of alumina and sapphire-like films: The anodization of aluminum is a large subject, beyond the scope of this brief review. In general, aluminum anodizes to form a porous film of amorphous (non-crystalline) phases alumina (boehmite, $\gamma$-alumina - $\mathrm{Al}_{2} \mathrm{O}_{3}$ ) on the aluminum. The film is composed of essentially the same materials-base as sapphire and alumina ceramics. These materials are among the most intrinsically hard known, and are also transparent. Remarkably, the size of the resulting porous structure is essentially unchanged from the parent aluminum. Aluminum is not removed - the incorporation of oxygen during anodization produces a molecular structure smaller than the original aluminum, thereby providing the driving mechanism to create pores in the anodized aluminum. Micro/Nano/porous alumina $\left(\mathrm{Al}_{2} \mathrm{O}_{3}\right)$ thin sheets or films exhibit a highly anisotropic and uniform pore structure consisting of channels perpendicular to the film surface, whose size and spatial distribution can be controlled by changes in the anodization process parameters 89,10 . The channel position is quasiregular, on a quasi-regular hexagonal matrix. The areal density, size and regularity of these pores is controllable over a remarkably wide range with the electrolyte chemistry, temperature, current density, 
and the electric field during the anodization ${ }^{11}, 12,13,14,15$. The pores typically range in size from $\sim 5 \mathrm{~nm}$ $200 \mathrm{~nm}$ in size, and vary in areal open pore density from $<1 \%$ of the alumina surface up to $\sim 68-70 \%$. The pores terminate in a non-porous thin boundary layer of alumina at the aluminum alumina interface, the thickness of which is also determined by the anodization conditions. Figure $5 \mathrm{a}, \mathrm{b}$ shows a schematic of the anodization process and an SEM of an anodized aluminum film produced by this process(see refs in figs). These porous structures have wide application in technology (filters for example), and are the basis of colored anodized aluminum by incorporation of dyes into the pores.
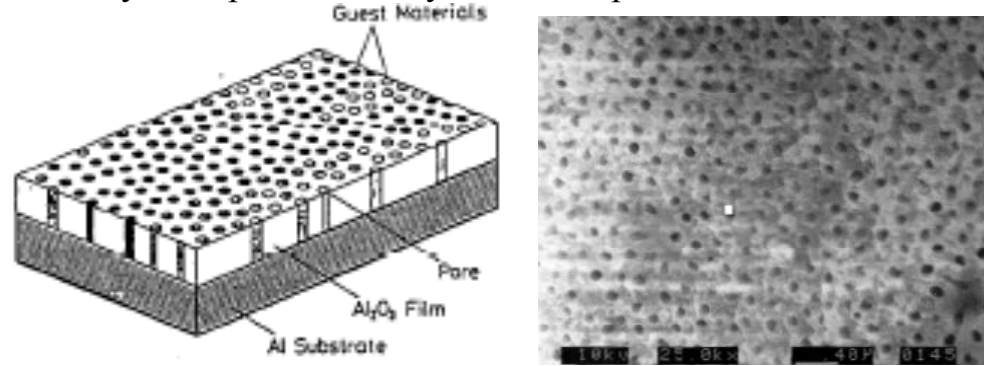

Figure 5a(L): Schematic of an anodized aluminum film produced by a total immersion method. ${ }^{\mathbf{1 6}}$ Figure $\mathbf{5 b}$ : SEM of a porous alumina film, produced by the edge anodization technique (EA). The nanoscale of the resulting hard film is evident. The fiducial is $400 \mathrm{~nm}$ in size and the pores are $\sim 50-100 \mathrm{~nm}$ in size, smaller than relevant optical wavelengths. ${ }^{17}$

The physics behind the Edge Anodization technique (EA) is shown in Fig.6. As the aluminum piece is lowered into the bath, the resistance may be modeled as a parallel network of resistors. There are three distinct regions in the bath that characterize the resistor network. The first region is near the bath surface. This region will have the lowest resistance because new aluminum surface is constantly being introduced into the electrolyte. In fact, the only appreciable resistance presented by this region is the resistance of the native oxide present on the aluminum surface.

The second region lies below the bath surface where the initial solid alumina (A12O3) barrier layer has formed. This region will present the highest resistance in the cell for the thin layer anodization because this is where the low conductance oxide is thickest. The third region is the porous alumina region which will have a resistance intermediate to the top two regions because the barrier oxide at the bottom of the pores is thinner than the oxide in the second region.

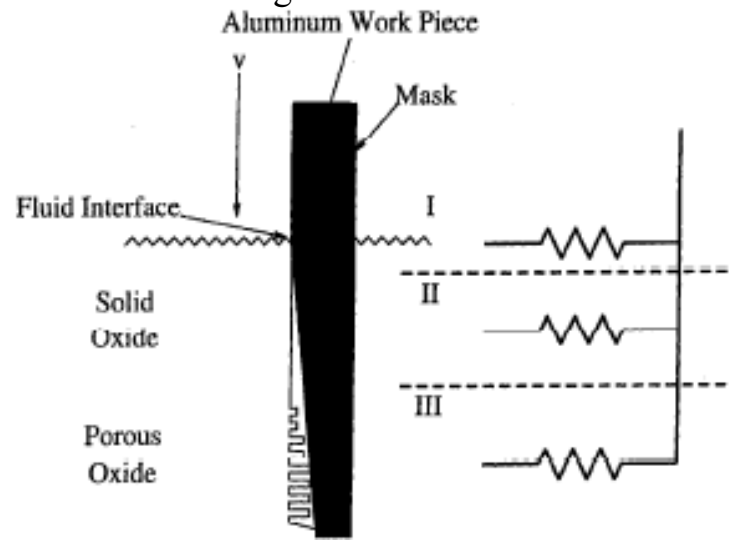

Figure 6: Schematic illustration of the anodization cell resistance. Note the three distinct regions on the sample representing the three stages of anodization as discussed in the text.

As the sample is lowered into the bath the size of the first region will remain unchanged. The velocity of the sample entry will determine the size of the other 2 regions. Higher velocities will allow the solid barrier region to be larger since we have determined that a finite time is required for the intial oxide barrier to reach its maximum thickness. The higher the initial velocity of the sample, the larger this region 
will become because more of the sample will be introduced during the period of time required for the barrier to reach maximum thickness. Conversely, lower velocities will result in a smaller solid barrier region.

To first order, the size of the porous region will depend on the overall size of the area A to be anodized. Large area samples will inevitably have a porous region develop near the leading edge of the sample first introduced into the bath. The size of this region is also dependent on the sample velocity to second order, since the size of this region is the size of the entire submerged portion of the sample, less the first and second regions. That is, for long times $t$ :

$$
A_{\text {porous }}=A_{\text {submerged }}-A_{\text {interface }}-A_{\text {barrier }}(1)
$$

or, by substituting the size of the other regions

$$
\mathrm{A}_{\text {porous }}=\left(\mathrm{v}_{\mathrm{tw}}-\mathrm{v}\right) \mathrm{t}_{\mathrm{req}} \mathrm{W}-\mathrm{A}_{\text {barrier }},(2)
$$

where $\mathrm{v}$ is the velocity, $\mathrm{w}$ is the sample width at the electrolyte surface, and $t_{\text {req }}$ is the time required for the initial oxide barrier to reach its maximum thickness.

We have demonstrated this EA technique to obtain complete anodization of aluminum on glass, with sputtered and with evaporated aluminum films, varying from about $0.5-4 \mu \mathrm{m}$ thick, roughly following the schematic of Fig. 5,6. A stepper motor was programmed with step size and step period to insert the slide into an anodization bath, starting from the bottom, and a programmable power supply was programmed with a current profile.

Figure 7a shows a photograph of an early test of an aluminized glass slide after anodization, and back illuminated. There are 4 regions from top to bottom, corresponding to 3 different anodization regions. The 3 regions starting from the bottom were created by 3 different insertion velocities (stepsize/step periods) and cell voltages.
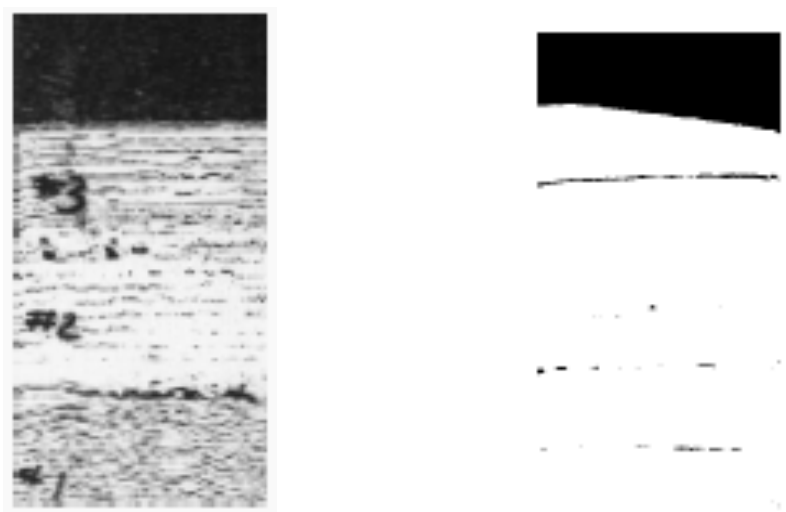

Fig.7a (left): Back-illuminated photograph of an aluminized and then edge anodized glass slide in early tests of edge anodization technique (EA), in 3 regions starting from the bottom. The 4th top region shows the opaque unanodized aluminum film used as the edge anode contact, as in Fig.2. The region second from the bottom (labeled with hand drawn " \#2 ") demonstrates nearly complete anodization but shows that the stepper is not being run smoothly enough. Regions 1 and 3 were anodized with rates and currents which left larger and more numerous islands of aluminum unconverted to alumina. The wavy aluminum lines are due mainly to the unstable creep of the meniscus (or wetting line) as the work piece is inserted into the electrolyte anodization bath.

Fig.7b (right): Photograph of a back-illuminated (resting on a light box) aluminized glass slide in tests of complete edge anodization (ET), in 6 regions with different current density and insertion rates, starting from the bottom. The top region shows the opaque unanodized aluminum film used as the edge anode contact, as in Fig.2. Nearly all of the aluminum below it was converted to transparent alumina. At 4 of the boundaries between regions where the stepper changes speed and the current is changes, some aluminum lines are left. A hardened steel scribe did not scratch these surfaces, whereas a carbide scribe scratched. 
The opaque top region shows the unanodized aluminum which was not inserted into the bath. The region second from the bottom shows evidence of nearly complete anodization of the aluminum. The banded regions at the bottom and third from the bottom reflect the stepper step size and anodization and insertion speed not optimal for complete anodization. Fig.7b shows the results of refined programming of the EA rig, showing nearly complete anodization in 6 regions of different EA conditions - the boundaries of some regions are nearly invisible.The transparent hard films appeared to be highly scratch resistant with metal tools, and required a carbide scribe. At some larger thicknesses, coloured interference fringes were evident. Angular reflection and transmission data taken with a HeNe laser and a Si detector indicated $\mathrm{n}<1.4$.

Figure 7c shows a photograph of a free-standing alumina film created by edge anodization of a standard aluminum foil about $10 \mu \mathrm{m}$ thick obtained from a local supermarket. It was anodized completely over a length of about $2.5 \mathrm{~cm}$ from the far end. To demonstrate its flexibility and clarity, it was bent or curled to rest conformally against a curled dollar bill and flash photographed. George Washington's face is clearly seen through the anodized foil end. The index was measured to be $\mathrm{n}=1.37$.

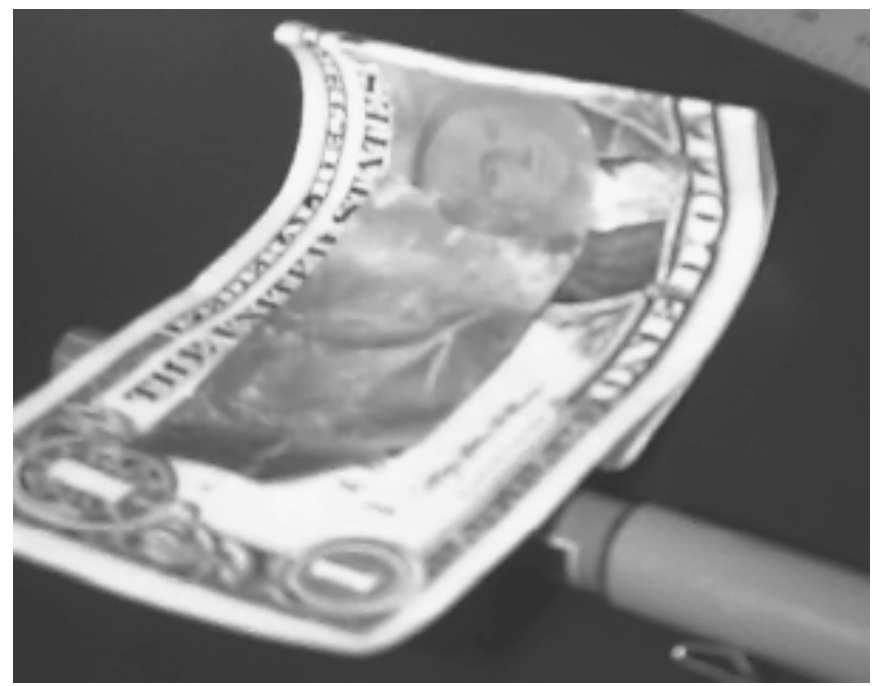

Figure 7c: Photograph of a George Washington's head on a dollar bill through a piece of free-standing alumina film, created from a strip of off-the-shelf Alcoa aluminum foil about $4 \mathrm{~cm}$ wide and $\sim 10 \mathrm{~cm}$ long. The clear anodized film area is about $\sim 2.5 \mathrm{~cm}$ long $\mathrm{x} \sim 3 \mathrm{~cm}$ wide starting at the edge of George Washington's face, covering his whole face and neck. The unanodized aluminum strip attached to it blocks an area $\sim 5 \mathrm{~cm} \mathrm{x} 3 \mathrm{~cm}$ starting at the right shoulder of the president and nicking the oval surrounding the " 1 " in the lower left of the picture. A pen is included for scale.

Such an immersion technique (EA) to form transparent nanoporous alumina films can be made to work on Al films deposited on fibers or plates provided: (i) sufficiently large aluminization "bell jar" is obtained to aluminize the work piece- such bell jars are common for glazing at least in sizes of 4' $\mathrm{x}$ 8', or tubular for fiber pulling; and (ii) sufficiently large anodization bath is obtained in which to edge immerse the work piece.

A possible disadvantage of the immersion technique is that the pores formed at the leading edge of the work-piece are guaranteed to be deeper and/or wider than those formed near the solution interface because they are exposed to the low $\mathrm{pH}$ electrolyte longer, and etch. The extent of this non-uniformity is not known and needs study. We predict that the anodizing of the pores shuts off once the aluminum is removed, and predict further removal of the alumina by the chemistry of the bath would not be a serious problem for work pieces $\sim 1 \mathrm{~m}$ or less. However, if the pores at the start begin to become too large and 
then merge during the process, the piece would periodically be withdrawn after a set distance of immersion and the pores protected from the anodization bath by a spin-on polymer (as used in VLSI processing) which could then be stripped off after full processing. The piece would then be re-immersed to the edge of the protected region (i.e. back to the bare aluminum film) and anodization continued. For a fiber work-piece, the lower end of the fiber could simply be guided to exit the bath after a sufficient anodizing time. A more complicated remedy for large plates would be a form-fitting elastic-lined flap/slot at the bottom of the tank, allowing entrance to a much diluted anodizing bath below the anodizing bath. We emphasize that a post-anodizing isotropic etch (phosphoric acid or similar) can widen the pores so that porosity exceeding $70 \%$ is possible.

Application of Edge Anodization(EA) of Aluminum Films to Form Transparent Ultra-Low Index Cladding Hard Coatings on Scintillating/Cerenkov Plates:

The application of the EA to transparent hard coatings on flat optical substrates is straightforward. Aluminum films of a few microns thickness are applied industrially to large area materials in sheets, films and highly complex shapes, most commonly using e-beam evaporators, and has been successfully scaled to continuous line production (eg mylar party balloons etc). Aluminum has a low melting temperature compared transparent with hard coatings, and adheres strongly to many clean plastic surfaces, including lexan and lucite and other polycarboates and acrylics. The aluminum is then converted by the EA to alumina/alumina-like materials. The alumina film produced by EA is essentially an amorphous sapphire or ceramic, one of the hardest materials. The Hardness of these materials varies between $10-20 \mathrm{GPa}^{18} \mathrm{On}$ hard substrates, the Knoop Microhardness of $\mathrm{Al}_{2} \mathrm{O}_{3}$ films is quoted ${ }^{19}$ typically as $\sim 1,000 \mathrm{~kg} / \mathrm{mm}^{2}$.

Application of Edge Anodization(EA) of Aluminum to Form Cylindrical Transparent Ultra-Low Index Cladding Hard Coatings on Scintillating, Cerenkov or WLS Fibers or Capillaries:

Coating thin films of $\mathrm{Al}$ and many other materials on fibers, or on the inside or outside of capillaries is standard practice (flow-through MOCVD, ALD, or flood MBE, and various forms of PVD, often with rotating susceptors). The growth of parallel pores on a cylindrical surface by EA is a possible issue. There are 2 points:

(a) The pores do follow the electric field, which in the fiber/capillary case will be radial - this is ok - if the pores are slightly further apart at the outside over the $3+$ micron thick film, it makes little difference in the index.

(b) The thickness of the aluminum film applied to the fibers is, say, $\geq 3$ micron thick. If the fiber or capillary cores are minimum of $150 \mu \mathrm{m}$, then the E-field varies by $\sim 2 \%$ or less over the thickness to be anodized, and locally the E-field is essentially flat to a few $\%$.

Adhesion and Surface finish: Adhesion of the alumina film may be an issue. On glass or quartz, this did no seem to be an issue (Figs 7a,b). The pores are fine enough that scratches to the surface of the pores is negligible, on the scale of optical wavelengths.

\section{REFERENCES}

${ }^{1}$ H.Leutz, Scintillating Fibers, NIM, Vol 364, 422 (1995)

2 - C.R.Martin, Science, Vol. 266, 1966 (1994)

- M.Saito et al., J.Applied Physics 75,4744 (1994)

- S. Nakuramura et al., Jpn.J.Appl.Phys.,Vol. 31, 3589 (1992)

- F.Keller, M.Hunter, D.Robinson, Structural Feature of Oxide Coating on Al", J.Electrochem.Soc.100, 411 (1953)

${ }^{3}$ J.Zhang et al., Materials Chem. \& Phys. 122395 (2010)

${ }_{5}^{4} \mathrm{http}: / /$ www.mpi-halle.mpg.de/department2/research-areas/ordered-porous-

${ }_{6}^{5}$ M Kokonou et al 2005 Nanotechnology 16 103-106

${ }^{6} \mathrm{http}: / /$ www.seas.ucla.edu/ pilon/OpticsNanoporous.html 
${ }^{7}$ For example: Patent title: Zero-order diffractive filter Inventors: Harald Walter Gilbert Gugler Alexander Stuck Robert Beer Marc Pauchard Agents: WEINGARTEN, SCHURGIN, GAGNEBIN \& LEBOVICI LLP Assignees: Origin: BOSTON, MA US IPC8 Class: AG02B2742FI USPC Class: 359558

${ }^{8}$ C.Foss et al., J.Phys.Chem. 96, 7497(1992) and loc.cite.

$9 \quad$,ibid. 98, 2963 (1994)

${ }^{10}$ A.Despic and V.Parkhutic, in Modern Aspects of Electrochemistry, Vol.20, Chap.6 (1989) J.Bockris et al., Eds., Plenum, NY

${ }^{11}$ C.R.Martin, Science, Vol. 266, 1966 (1994)

${ }^{12}$ M.Saito et al., J.Applied Physics 75,4744 (1994)

${ }^{13}$ S. Nakuramura et al., Jpn.J.Appl.Phys.,Vol. 31, 3589 (1992)

14 ibid.

${ }^{15}$ F.Keller, M.Hunter and D.Robinson, Structural Feature of Oxide Coating on Aluminum", J.Electrochem.Soc. 100, $411(1953)$

${ }_{16}$ C.Alpert et al., J.Am.Ceram.Soc. 71, C-371 (1988)

${ }^{17}$ A.Memarian et al., J.Vac.Sci.Tech. A3, 2434 (1985)

${ }^{18}$ C.Alpert et al., J.Am.Ceram.Soc. 71, C-371 (1988)

${ }^{19}$ A.Memarian et al., J.Vac.Sci.Tech. A3, 2434 (1985) 


\section{Monolithic Pixel Sensors}

The standard figure of merit for vertex tracking accuracy is the accuracy on the impact parameter, $\mathrm{S}_{\mathrm{IP}}$, defined as the distance of closest approach of the particle track to the colliding beam position. This can be parametrised as: $\mathrm{s}_{\mathrm{IP}}=\mathrm{a}+\mathrm{b} / \mathrm{p} \sin ^{\mathrm{k}} \mathrm{q}$, where $\mathrm{p}$ is the particle momentum, $\mathrm{q}$ the track polar angle and $\mathrm{k}$ $=3 / 2$ for the R-f and $5 / 2$ for the $\mathrm{z}$ projection. The identification of hadronic jets originating from heavy quarks is best achieved by a topological reconstruction of the displaced vertex structure and the kinematics associated to their decays. The ability in reconstructing these sequences of primary, secondary and tertiary vertices depends on the impact parameter resolution. This is related to the single point resolution of the sensors and their geometry. The figure of merit for the resolution is the distribution of the impact parameter values for charged particles originating from $b$ hadron decays. It is useful to recall that the impact parameter does not depend, to a first approximation, on the $b$ hadron energy, since the angle of production of the track in the lab frame is proportional to (bg) ${ }^{-1}$, which compensates the bg term in the $b$ hadron decay length. The average impact parameter value is $\sim 220 \mathrm{~mm}$, with a $0.15(0.24)$ fraction of tracks having impact parameter below $15(35) \mathrm{mm}$. The asymptotic resolution a is typically proportional to the sensor point resolution, the tracking lever arm and, to a lesser extent, the radial position, $\mathrm{R}$, of the first layer. The multiple scattering coefficient, $\mathrm{b}$, scales with sqrt(d) $R / p$, where $d$ is essentially the material thickness of the beam pipe and the first layer. The tracking lever arm is constrained by detector volume, number of electronics channels and cost. The minimum detector radius is typically defined by radiation or particle density conditions from particle fluence and beam-induced backgrounds and it is accelerator dependant. This was 63mm at LEP and $25 \mathrm{~mm}$ at SLC, is $30-50 \mathrm{~mm}$ at the LHC, with plans to further reduce it, and should be $15-30 \mathrm{~mm}$ at future lepton colliders.

The two main drivers for the sensor pixel pitch, $\mathrm{P}$, are single point resolution, scaling as $\mathrm{P}$ and local occupancy, scaling as the pixel area (i.e. proportional to $\mathrm{P}^{2}$ for square pixels) times the readout time. As a result of this, starting with the current LHC detectors, fast time-stamping, required by occupancy, has become part of the overall optimisation and the space-time granularity has $t$ be considered as the appropriate parameter space for characterising any future vertex detectors.

Monolithic pixel sensors, where the Si sensitive volume and the charge sensing and possibly part of the readout circuitry are implanted in the same Si wafer, have emerged at the end of the '90s in the framework of the R\&D towards a future linear collider as the way forward towards thin Si sensors with very high granularity, well beyond the technological capabilities for the hybrid pixel sensors the under development for application in the LHC experiments. This development was made possible by the progress in deep sub-micron CMOS processes and the availability of commercial processes with thick epitaxial layers aimed at sensors for optical imaging and consumer digital cameras.

Monolithic pixels offer less mass than hybrid pixel detectors, lower capacitance and a single chip solution without bump bonding $\backslash$ cite $\{$ Fossum1997, Turchetta2001\}. The fact that monolithic CMOS pixels combine particle detection and (at least part of their) front-end electronics (FEE) in the same device is a prominent advantage, but turns into a limitation as their industrial manufacturing relies on procedures optimised for commercial items which may depart substantially from those needed for charged particle detection. Howevere, CMOS industry has evolved in a direction which allows CMOS pixels to progressively approach their real potential. Developments towards future vertex detector systems rely on the further advances in the CMOS technology to cope with the demanding requirements of future collider experiments. To this purpose, many R\&D paths are being pursued in the vertex detector community, with the US playing an important, although not a leading, role The shrinking of the feature size of CMOS transistors is the most apparent outcome of the evolution of 
microelectronics. This is being exploited in the design of new pixels where advanced functions can be performed in the circuitry implemented in each pixel to provide the required signal-to-noise ratio and to handle the high data rate. These functions include amplification, filtering, calibration, discriminator threshold adjustment, data sparsification and time stamping.

CMOS sensors for particle tracking were initially designed following the classical guidelines of the NMOS-only 3-transistor cell of image sensors to collect the charge generated in an almost field-free epitaxial layer. Now, thanks to CMOS scaling, more functions are being included in the pixel cell, including correlated double sampling and hit binary information from a discriminator. This leads itself to achieve zero suppression at the pixel level, which is a highly desirable feature given the large number of channels foreseen and the fast readout of the pixel matrix. New strategies are also being devised in terms of the needed readout architecture, introducing a higher degree of parallelism to speed up the transfer of information from pixels to the chip periphery. Another way to comply with a high hit rate is to adopt the solutions developed for hybrid pixel sensors. This requires that the classical continuous-time analog signal processing chain (charge-sensitive preamplifier, shaping filter) is integrated in the pixel cell, together with the digital blocks needed for a high-speed sparsified readout of the matrix $>$ cite $\{$ Traversi:2007\}. The implementation of complex circuitry in the pixel cell requires a technique to insulate the transistors from the collection volume, to avoid parasitic charge collection. This can be done using large deep $\mathrm{N}$-well electrodes as sensing elements or by profiting of the thin oxide insulating layer of the silicon-on-insulator (SOI) technology $\backslash$ cite $\{$ Kucewicz2005,Arai2010\} to use full CMOS capabilities, without degradation of the charge collection efficiency.

These technologies make it possible to implement advanced readout architectures, that can be tailored to different applications. However, special care needs to be taken to avoid analog-digital coupling. In the last few years, successful results have been reported for the 130nm CMOS technology node $\sim$ cite $\{$ Neri:2010\} and the $200 \mathrm{~nm}$ SOI process $\sim$ cite $\{$ Arai2010\}. Recently, the 180nm CMOS process featuring a nearly $20 \mathrm{~mm}$ thick epitaxial layer with resistivity exceeding $1 \mathrm{~kW} \mathrm{~cm}, 6$ to 7 metallisation layers and additional P-wells offers the possibility to use both types of transistors inside the pixels thus allowing for a substantial increase of the in-pixel micro-circuit complexity /cite $\{$ Zucca2012\}. The 90nm and 65nm CMOS technology nodes are currently being evaluated as a promising solution to the integration of high density circuitry and high speed functionalities in a small pixel. High-voltage CMOS pixels $\sim$ cite \{peric2011\} were pioneered several years ago with a larger feature size process $\sim$ cite $\{$ Kenney 1994$\}$ and their development is currently pursued on smaller feature size in the perspective of tracker upgrades at LHC.

It is worth mentioning that, besides device scaling, advanced CMOS technologies bring along other desirable features that pixel R\&D projects are presently exploring. Metal interconnections are isolated by low-k dielectrics to reduce parasitic capacitance and time constants of transmission lines. This may be exploited by minimising the electronic functions in the pixel cell and by connecting each pixel to signal processing blocks in the chip periphery. Thanks to the very high integration density of the CMOS process, this peripheral readout strategy may require just a small silicon area outside the pixel matrix itself. New strategies are also being devised in terms of the needed readout architecture, introducing a higher degree of parallelism to speed up the transfer of information from pixels to the chip periphery.

Monolithic CMOS and DEPFET technologies offer us sensors with thin active layers, pixel cells of $\mathrm{O}(10 \mathrm{x} 10) \mathrm{mm}^{2}$ in size with a typical capacitance of $\mathrm{O}(1) \mathrm{fF}$ and 10-40 e ENC of noise and significant data processing capabilities implemented in-pixel or on-chip. An active thickness of $15-20 \mathrm{~mm}$ is sufficient for achieving a detection efficiency $>99 \%$ with CMOS pixels, even at operating temperatures in excess to $40^{\circ}$. Back-thinning technologies, such as grinding and chemical etching now commonly 
available as commercial service, have been successfully applied to obtain 40-50 mm-thick CMOS sensors for the STAR HFT at RHIC ${ }^{\mathrm{ii}}$ and in the R\&D for linear colliders ${ }^{\mathrm{iii}}$ and B-factories ${ }^{\text {iv }}$. Finally, the monolithic pixel technology is gaining ground in applications at the LHC, for example in the ALICE upgrade where the baseline design is based on monolithic pixels to obtain ladders with an overall material budget $0.3-0.4 \% \mathrm{X}_{0} /$ layer and the sensor contributing $\sim 15 \%$. DEPFET sensors can be thinned to less than $100 \mathrm{~mm}$ in the sensitive region, using wafer bonding technology, retaining a frame which ensures sufficient stiffness of the mechanical module ${ }^{\mathrm{vi}}$. Thin DEPFETs are currently under development for BELLE-II, where the target thickness is $75 \mathrm{~mm}$, and first results have already been obtained for prototypes successfully thinned to $50 \mathrm{~mm}^{\mathrm{vii}}$.

The use of a moderate- to high-resistivity substrate is the latest step in the process development exploited by monolithic pixel sensors. The use of a depleted sensitive layer enhances the amount of collected charge in monolithic pixel sensors and the collection time compared to the almost field-free epitaxial layer of the standard CMOS active pixel sensors, without compromising the sensor competitive thickness. Given that the reverse bias voltage, $\mathrm{V}_{\mathrm{d}}$, required to depleted a given thickness $d$ scales as $d^{2} / \mathrm{r}$, where $\mathrm{r}$ is the Si resistivity, a modest $\mathrm{V}_{\mathrm{d}}$ of $\mathrm{O}(10 \mathrm{~V})$ is sufficient to fully deplete a thin layer of high resistivity Si. Operating the detector fully depleted has advantages both in terms of signal charge and of collection time, thus improving the performance after irradiation by more than an order of magnitude. This can be achieved either with specialised CMOS processes featuring a resistivity in excess of $1 \mathrm{~kW} \mathrm{~cm}$, or with the SOI process on high resistivity handle wafer. Both approaches have been successfully exploited in monolithic pixel R\&D ensuring signal-to-noise values of 30-40 from minimum ionising particles at room temperature. The beam telescope developed within the EUDET EU project uses sensors based on high resistivity CMOS pixels thinned to $50 \mathrm{~mm} \sim$ cite $\{$ Aguilar:2012ez . They provide a $\sim 3 \mathrm{~mm}$ single point resolution at a rate of $\sim 10^{4}$ frames/s over an active surface of about $2 \mathrm{~cm}^{2}$ paved with 670,000 pixels and can cope with a flux of particle exceeding $10^{6} / \mathrm{cm}^{2} \mathrm{~s}$. The SiliconOn-Insulator process with an high-resistivity handle wafer, developed by an international consortium to which both LBNL and FNAL contribute significantly, offers further appealing opportunities by removing the inherent limitations of bulk CMOS processes for the polarity of the transistors employed in the pixel cell. After the pioneering effort in a $3 \mathrm{~mm}$ CMOS process of $\sim$ cite $\{$ krakow $\}$, a commercial $0.2 \mathrm{~mm}$ SOI process has been made available since a few years by a collaboration of KEK with Lapis Semiconductor Inc. (formerly OKI Semiconductors), Japan, $\sim$ cite $\{$ soi\}. The high resistivity sensitive volume ensures large signals with no interconnections, low collection electrode capacitance and a lowpower, potentially radiation tolerant device $\backslash$ cite $\{$ Battaglia:2007eq,soi2\}. The R $\backslash \& D$ has successfully solved the transistor back-gating problem. Prototype SOI pixels recently tested with a $200 \sim \mathrm{GeV} \mathrm{p}$ beam at CERN have been successfully operated with a depletion voltage up to $70 \sim \mathrm{V}$, giving a preliminary result of $(1.2+/-0.05) \mathrm{mm}$ single point resolution and particle detection efficiency above 98\% /cite $\{$ soi-pixel2010\}.

Based on a more specialised technology, DEPFET pixels have been developed since the late 80 's $\sim$ cite $\{$ Lutz1987\} and integrate a p-MOS transistor in each pixel on the fully depleted bulk. Electrons, collected in the internal gate, modulate the transistor current. Their low input capacitance ensures low noise operation and makes them suitable for a broad range of applications from collider detectors $\sim$ cite $\{$ Rummel:2010\} to X-ray astronomy $\sim$ cite $\{$ Stefanescu2010aa $\}$.

The design of the electronics for monolithic pixels has to be adapted to the limited amount of collected charge and the need to minimise power. In principle, for monolithic pixel sensors collecting charge from the thin epitaxial layer, the $\mathrm{Q} / \mathrm{C}$ ratio and power consumption might not be better compared to hybrid pixel detectors. The reduction in power consumption and $\mathrm{Q} / \mathrm{C}$ improvement can be achieved by minimising $\mathrm{C}$, decreasing the size of the collection electrode, and by increasing the charge collection 
and depletion depth, by applying reverse bias voltage with a moderate- to high-resistivity substrate. Analog and digital power consumption typically differ by less than an order of magnitude in pixel detectors for HEP applications. Analog power reduction by an improved Q/C or by power pulsing has therefore to be matched by a similar reduction in digital power. Power pulsing schemes also for the digital readout are considered. However also architectures limiting the off-chip data rate and minimising the number of operations are under study. As an example, in the LePix project pursued by an European collaboration centred at CERN $\sim$ cite $\{$ Mattiazzo2012\}, the in-pixel circuitry is reduced to minimise $\mathrm{C}$ and the data from different pixels is combined in different projections and immediately transmitted to the readout electronics in the periphery of the chip. The different projections are chosen carefully to minimise ambiguities and for a practical example with 4 projections, simulations indicate that occupancies of $\sim 50 \sim$ hits $/ \mathrm{cm}^{2}$ can be handled while only $4 \mathrm{~N}$ signals need to be treated at the periphery for an $\mathrm{NxN}$ pixel matrix. An important component of the power consumption is the offdetector data transmission, and any progress to optimise power/data rate in this area will be beneficial to almost all new applications.

$3 \mathrm{D}$ integration technologies promise to provide a very elegant way for implementing advanced readout architectures of a pixel matrix $\sim$ cite $\{$ Yarema2010\}. It offers a solution to the conflict between high pixel granularity and fast readout requirements by integrating heterogeneous technologies with individual tiers dedicated to charge collection and the others devoted to signal processing with dedicated architectures. Digital electronics can be removed from the silicon layer where the sensing electrodes and the analog front-end circuits are located, eliminating interference due to substrate coupling. In a purely digital layer, the designer is allowed to integrate various advanced readout concepts, such as time-ordered readout of hit pixels, in a "data push" or triggered way, enabling a pixel matrix to deal with a hit rate of $100 \mathrm{MHz} / \mathrm{cm}^{2}$ with a readout efficiency close to $100 \% \sim$ cite $\{$ Gabrielli2011\}.

There are several approaches to 3D integration differing in terms of the minimum allowed pitch of bonding pads between different layers and of vertical TSVs across the silicon substrate. The HEP community focused a large effort on the design of 3D integrated circuits with the "via first" technology, where the TSVs are etched in the silicon wafers in the early stages of CMOS fabrication $\sim$ cite $\left\{\right.$ Yarema2010\}, provided by Tezzaron/GlobalFoundries ${ }^{1}$. Even though this has only led to a fully functional device after several cycles, it remains a very promising approach. However, even with less aggressive 3D technologies, such as the so-called "via last" processes where TSVs are fabricated on fully processed CMOS wafers, a significant advantage can be gained in most cases, as only one or two connections are needed between the analog and digital blocks of a single pixel cell, and the digital layer can use low-density peripheral TSVs to reach backside bonding pads for external connection. Devices based on the "via last" technique are being studied within the AIDA EU project $\sim$ cite $\{$ Traversi2012\}.

Sensors of the thickness achievable with monolithic technologies and back-thinning, $50 \mathrm{~mm}$, are compatible with achieving a material budget of $\sim 0.1 \% \mathrm{X}_{0} /$ layer, needed to meet the value of $\mathrm{b} \sim$ $10 \sim \mathrm{mm} / \mathrm{GeV}$ for the multiple scattering term in the impact parameter resolution, identified as a requirement at a linear collider and also the b-factory requirements. For sensors of this thickness, the ladder support is of major importance for counteracting the chip warping and insuring the module stability and planarity. In the design of thin ladders for applications at B-factories, STAR and the linear collider with thin sensors, as well as those for an LHC upgrade, the Si ceases to be the largest single factor in the overall layer material budget which becomes the cable routing signals, power and clocks.

1 Tezzaron Semiconductor, Naperville, IL, USA; GlobalFoundries Inc.\Milpitas, CA, USA 
Therefore, in HEP applications there seems to be limited interest in pushing the sensor thickness below $\sim 50 \mathrm{~mm}$. In order to progress towards modules with overall smaller total material budget, sensor stitching, with clocks and signals routed on metal lines in the chip, appears a promising path. The development of very light double-sided ladders, which are advantageous mechanically and offer hit correlation to reject low energy particles from beam background, appears promising. The PLUME collaboration is addressing some of the challenges related to ladder based on thin monolithic pixel sensors ${ }^{\text {viii }}$.

i $\quad$ M. Winter, Nucl. Instrum. Meth. A623 (2010) 192.

ii $\quad$ L. Greiner et al., Nucl. Instrum. Meth. A650 (2011) 68.

iii M. Battaglia et al., Nucl. Instrum. Meth. A579 (2007) 675.

iv $\quad$ G. Rizzo et al., Nucl. Instrum. Meth. A650 (2011) 169.

v ALICE Collaboration, CERN-LHCC-2012-012.

vi L. Andricek et al., IEEE Trans. Nucl. Sci. NS 51 (2004) 1117.

vii P. Fischer et al., Nucl. Instrum. Meth. A582 (2007) 843 and C. Marinas et al., JINST 7 (2012) C02029.

viii A. Nomerotski et al., Nucl. Instrum. Meth. A650 (2011) 208. 


\title{
Snowmass 2013 White Paper \\ Novel Compact Accelerator-Based Neutron and Gamma Sources for Detector Calibration
}

\author{
G. Jennings, C. Sanzeni, D.R. Winn* \\ Fairfield University, Fairfield CT 06824
}

*Corresponding Author winn@fairfield.edu 1+203.984.3993

\begin{abstract}
Novel ultra-compact, electrically switchable/pulsed, 1-14 MeV-level neutron and photon generators that have application embedded into large detector systems, especially calorimeters, for calibration. The small sizes are applicable to designed in-situ deployment, or by insertable channels into large high energy physics detector systems. For testing of prototypes, or for detector module production tests, these devices are convenient for lab bench tests.
\end{abstract}

\section{Introduction:}

Calibration of large experiments is an ongoing issue in HEP experimentation. Convenient sources of radiation which could be turned on or off remotely, with temporal modulation, and when off have little or no radiation, offer distinct advantages, especially over radioactive sources which must be mechanically inserted (for example, the source wires used in the CMS hadron calorimeter), and which have no timing properties. Much larger neutron generators have been used in off-line calorimeter calibration ${ }^{1}{ }^{2}$. We review two novel devices which have application in the energy, intensity and cosmic frontiers for high energy physics experiments.

Ultra-Compact Neutron Accelerator Source: Sandia National Labs(SNL) has announced a miniaturized neutron generator dubbed the "Neutristor", using ceramics with an ion drift region of a few $\mathrm{mm}^{3}$. The DT or DD neutristor operates in CW (for 100's hours) or pulsed operation, and produces up to $\sim 10^{4-5} 14.1$ $\mathrm{MeV}$ DT n/s, with a lower(x0.01) rate of $2.5 \mathrm{MeV}$ DD neutrons. The deuterium-tritium base reaction pulsed neutron generator is packaged in a flat computer chip shape of $1.54 \mathrm{~cm}(0.600 \mathrm{in})$ wide by 3.175 $\mathrm{cm}$ ( $1.25 \mathrm{in})$ long and $0.3 \mathrm{~cm}$ thick has demonstrated $10^{3}$ neutrons per pulse $(14 \mathrm{MeV})$ in a $500 \mathrm{~ns}$ pulse.

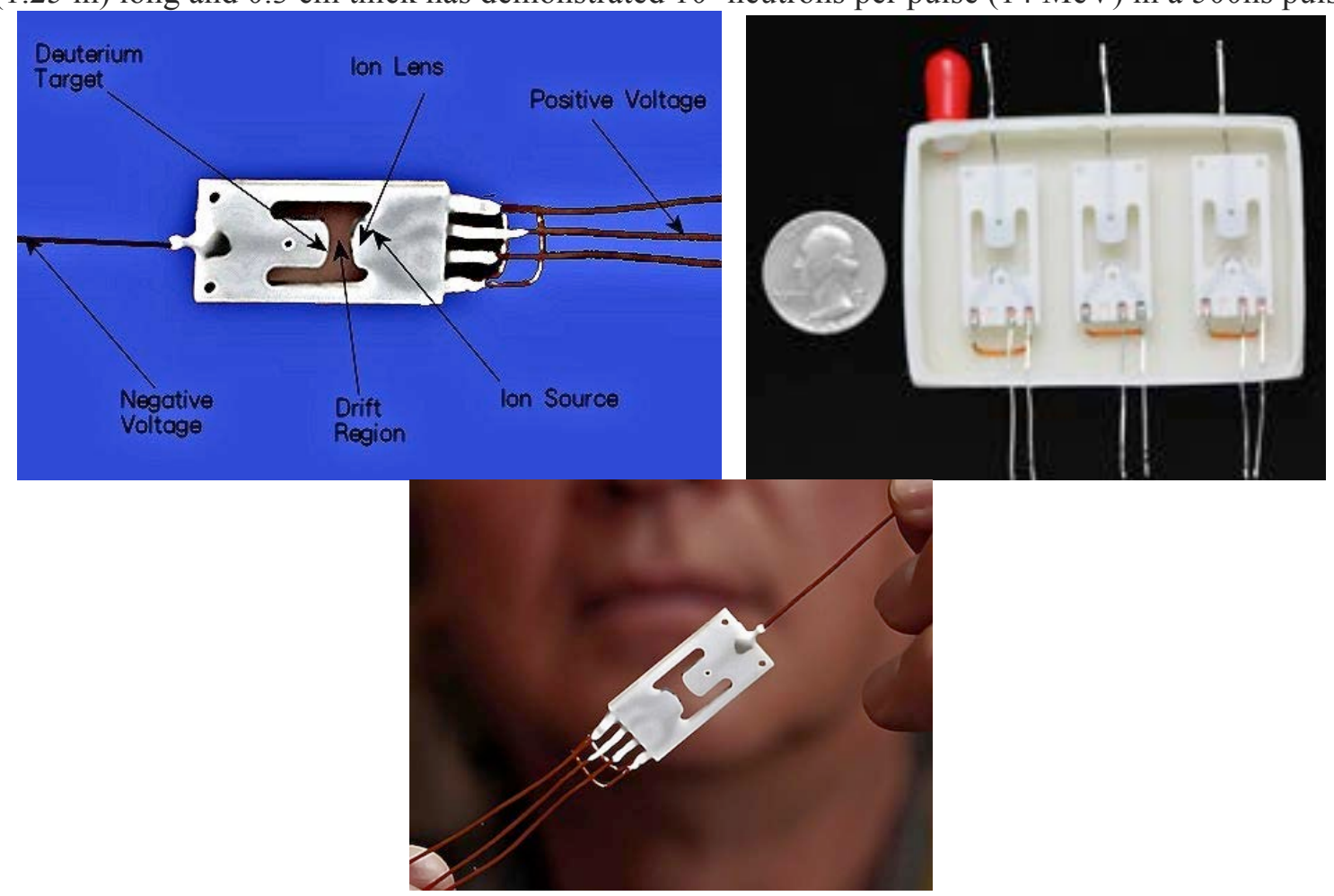

Figs 1: The Sandia Neutristor n-generators - size 1.25" x 0.6" x 0.13 " which generate 1000n per 500ns pulses. 
The Neutristor neutron generator is based on a deuterium ion beam accelerated to impact a tritium-loaded target. The accelerating voltage is in the $15-$ to $20-\mathrm{kV}$ range with a $3-\mathrm{mm}$ gap, and the ion beam is shaped by using a lens design to produce a flat ion beam that conforms to the flat rectangular target. The ion source is a simple surface-mounted deuterium-filled titanium film with a fused gap that operates at a current-voltage design to release the deuterium during a pulselength of $\leq 1 \mu \mathrm{s}$. The 4-terminal neutristor could easily be slotted into or assembled into large calorimeters or other detector systems, depending on the application, and switched on between fills in LHC, for example. The estimated production cost for neutristors is in the neighborhood of US $\$ 2,000$ and are assumed to be disposable if necessary. Sandia is working on neutristors $\sim 2$ orders of magnitude smaller, fabricated using MEMS.

Compact 11.7 MeV Gamma Source: Drs. Ka-Ngo Leung of Lawrence Berkeley Lab(LBNL) and Arlyn Antolak of Sandia Lab(SNL) have invented a novel neutron/gamma generator in which the electrostatic acceleration column is eliminated via a plasma generator ${ }^{4}$.

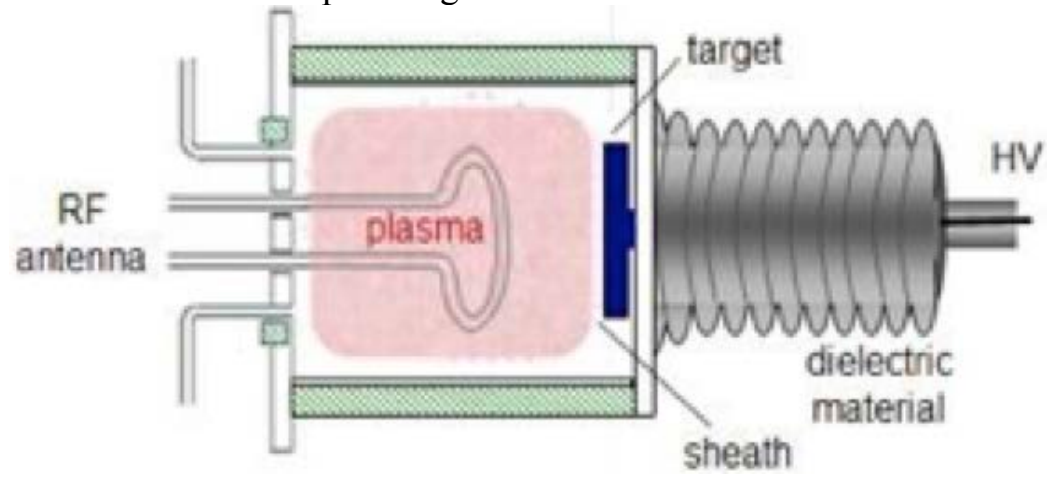

Fig 2: Schematic of a compact plama driven n/gamma accelerator, $5 \mathrm{~cm}$ long $x 1.5 \mathrm{~cm}$ diameter for generating $10^{6} 11.6 \mathrm{MeV}$ gamma/s.

Due to the elimination of the electrostatic accelerator column, the Berkeley neutron/gamma generator can be operated at higher pressures than previous D-T or similar neutron tubes, so it is ideal for sealed tube operation. As a result, the generator can be very compact in size - as small as 1.5 centimeters in diameter and 5 centimeters in length. When operated with $\mathrm{a} \mathrm{LaB}_{6}$ target the $\mathrm{p}+\mathrm{B}^{11}$ gamma source generates up to $\sim 1$ million $11.7 \mathrm{MeV}$ gamma per s, via the ${ }^{11} \mathrm{~B}(\mathrm{p}, \gamma){ }^{12} \mathrm{C}$ reaction. D-D, D-T, or T-T neutrons can be generated at high current density. The generator consists of a sealed chamber, an RF antenna that can be placed either inside the ion source chamber or on the external surface of the chamber wall, a gas source, the target, a power source, and permanent magnets surrounding the target to suppress electron-generated $\mathrm{x}$-rays. After a plasma is formed, a series of high voltage pulses are applied to the target, forming a plasma sheath that serves as an accelerating gap. For creating either a neutron beam or a gamma beam, the design is essentially the same, with changes in appropriate source gases and target material. Other gamma generators can be converted from neutron generators via the reaction ${ }^{19} \mathrm{~F}(\mathrm{n}, \mathrm{\alpha})^{16} \mathrm{~N}$, followed ${ }^{16} \mathrm{~N}$ decay yielding a $6.13 \mathrm{MeV}$ photon.

SUMMARY: Recent developments in ultra-compact, time-resolvable, sealed tube neutron and gamma generators are applicable to in-situ detector calibration, particularly calorimeters, and to testing prototypes and production modules, and merit development, especially for shorter than 500ns pulsewidths.

1 J. M. Bauer, Absolute Energy Calibration with a Neutron-Activated Liquid-Source, arXiv:physics/0312128

2 DR Winn, Calibration of High Energy Physics Detectors Using Neutrons Generated from Compact Accelerator D-D and D-T Reactions, IEEE Trans.Nuc.Sci. 33.1, 213 (Feb 1986)

${ }^{3}$ Elizondo-Decanini, J.M. et al., IEEE Trans. Plasma Sci.Vol. 40 Issue: 9

${ }^{4}$ http://www.lbl.gov/tt/techs/lbnl2456.html 


\section{The Next Generation of Photo-Detectors for Science in the Cosmic, Intensity and Energy Frontiers}

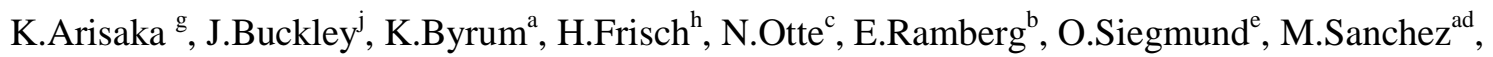
G.Varner ${ }^{\mathrm{i}}$, J.Va'vra ${ }^{\mathrm{f}}$, R.G.Wagner ${ }^{\mathrm{a}}$, M.Wetstein ${ }^{\mathrm{h}}$

Argonne National Lab ${ }^{\mathrm{a}}$, Fermi National Lab ${ }^{\mathrm{b}}$, Georiga Institute of Technology ${ }^{\mathrm{c}}$, Iowa State University ${ }^{\mathrm{d}}$, Space Sciences Laboratory at Berkeley ${ }^{\mathrm{e}}$, Stanford Accelerator National Lab ${ }^{\mathrm{f}}$, University of California at Los Angeles $^{\mathrm{g}}$, University of Chicago ${ }^{\mathrm{h}}$, University of Hawaii ${ }^{\mathrm{i}}$, Washington University ${ }^{\mathrm{j}}$

Photodetectors are an important component for experiments at all frontiers of science. To date, vacuum technology has been the only way to achieve large areas while maintaining high gains and photon counting operation. A R\&D program which includes new developments in photodetectors is crucial for HEP.

At the Energy Frontier, the development of large area photo-detectors capable of measuring time-ofarrival of relativistic particles with resolutions below a few picoseconds would provide new capabilities such as photon vertexing, multiple-interaction separation, and charge-particle identification an order of magnitude beyond what is achievable today. This is a crucial capability for the High Luminosity upgrade for the LHC, where several hundred interactions can pileup within $300 \mathrm{psec}$ in a single beam crossing.

Within the Intensity Frontier, both water based and liquid argon time projection chambers would greatly benefit from large area economical photodetectors with higher quantum efficiency and precision spatial and timing resolution. A vertexing capability for photons can be an important component for rare neutral kaon decay experiments, where the background from a decay into pi zero's can be significantly reduced by associating time-of-arrival of photons in a calorimeter with a pulsed incoming beam.

In the Cosmic frontier, both indirect and direct dark matter experiments will benefit from R\&D of new photodetector instrumentation. New economical large area and higher quantum efficiency photodetectors could provide huge savings to Cherenkov cameras. Radioactive free photodetectors would have a huge impact on direct dark matter experiments, such as liquid noble gas detectors.

A promising new approach combining modern semiconductor technology with vacuum devices has emerged in the last few years. For example, new cathode structures (in some cases semiconductor heterostructures engineered to promote efficient electron transport and other features such as intrinsic high gain or fast timing) are being combined with new kinds of electron multipliers that lend themselves to very large areas, and mass fabrication. Designs for these electron multipliers range from silicon and ceramic microchannel plates and semiconductor nanostructures to gas electron multipliers (GEMs) aimed at dramatically reducing the cost per unit area. New techniques for direct deposition of cathode surfaces on silicon microchannels could result in dramatic improvements. The funding for all of these developments falls short of what is required to maintain credible efforts at universities and national laboratories.

\section{Large Area Photo-detectors Using Microchannel Plate Technology}

The development of new large area photodetectors based on borosilicate glass capillary array microchannel plates (MCP) which have been functionalized via a process of atomic layer deposition promises to have a transformational effect on the realization of new economical large area photodetectors. 
This research is still in its infancy. However looking forward, continued R\&D for specific applications like high rate or purity environments as well as continued $R \& D$ on photocathode properties and quantum efficiencies is the next step to take advantage of the recent developments.

\section{Silicon Photomultipliers}

Semiconductor photon detectors are an attractive alternative to traditional vacuum tube based photomultipliers. Silicon Photomultiplier (SiPM), which are Geiger-mode APDs, are comprised of an array of avalanche photodiodes, typically $10^{2-4}$ per square millimeter. The SiPM offers many advantages such as low power, low voltage, compactness, excellent timing resolution, good linearity, robustness and insensitivity to magnetic fields.

\section{Hybrid Photomultipliers}

Hybrid photomultipliers such as those that merge a conventional bialkali photocathode with a gain structure such as focusing to a SiPM, MCP, or APD look to be useful for some specific applications or to solve a particular constraint for a detector. Hybrid Avalanche Photo Diodes with GaAsP high quantum efficiency photocathodes entirely made by synthetic fused silica (quartz) offers the promise of a photodetector free from any radioactivity. These photodetectors are well suitied for future direct dark matter and double beta decay experiments. Further research in to such types of detector would widen the alternatives available for experiments.

To summarize, participation of many HEP groups in the research and development of a new generation of photodetectors is currently underway. This R\&D will benefit all HEP science frontiers as well as US industry and other areas of science where photodetection is important. 


\title{
Snowmass White Paper
}

\section{Secondary Emission Calorimetry: Fast and Radiation-Hard}

\section{Fairfield University ${ }^{1}$ - University of Iowa ${ }^{2}$ - Argonne $^{3}$ Collaboration}

\author{
A.Albayrak-Yetkin ${ }^{2}$, B.Bilki ${ }^{2,3}$, J.Corso ${ }^{2}$, P.Debbins ${ }^{2}$, G.Jennings ${ }^{1}$, V.Khristenko ${ }^{2}$, A.Mestvirisvilli ${ }^{2}$, \\ Y.Onel $^{2}$, I.Schmidt ${ }^{2}$, C.Sanzeni ${ }^{1}$, D.Southwick ${ }^{2}$, D.R.Winn ${ }^{1}$, T.Yetkin ${ }^{2}$ \\ *Contact/PI - winn@,fairfield.edu \\ cell/txt/vm 1+203.984.3993
}

Abstract: A novel calorimeter sensor for electron, photon and hadron energy measurement based on Secondary Emission(SE) to measure ionization is described, using sheet-dynodes directly as the active detection medium; the shower particles in an SE calorimeter cause direct secondary emission from dynode arrays comprising the sampling or absorbing medium. Data is presented on prototype tests and Monte Carlo simulations. This sensor can be made radiation hard at GigaRad levels, is easily transversely segmentable at the $\mathrm{mm}$ scale, and in a calorimeter has energy signal rise-times and integration comparable to or better than plastic scintillation/PMT calorimeters. Applications are mainly in the energy and intensity frontiers.

Introduction: Secondary Emission ${ }^{1}(\mathrm{SE})$ is extraordinarily radiation resistant, and a fast process occurring in less than $1 \mathrm{~ns}$ in metal oxides. The SE beam monitors used at accelerators have no change in operation up to $10^{21}$ protons $/ \mathrm{cm}^{2}$ and use a simple native metal oxide film a few nm thick (alumina or titania) $)^{2}$. In PMT dynodes, the submicron thick metal-oxide coatings on the dynodes survive 10's GRad of heavily ionizing electron bombardment with tolerable degradation ${ }^{3}$. Essentially an SE calorimeter is composed of a monotonous array of PMT dynodes without a photocathode, which can self-operate at the Gain-BW of PMT, $\left(\sim 10^{6} \times 100 \mathrm{MHz}\right)$. Dynode arrays can cycle to air without degradation, such as those used in massspectrometry detectors ${ }^{4}$, and do not require thin film control as in photocathodes, making construction far easier and cheaper than PMT. Detectors in the forward regions at present and future colliders would greatly benefit from this device. Precision calorimetry at very high rates for lepton number violation would also benefit.

In a Secondary Emission (SE) calorimeter ionization detector module, Secondary Emission electrons (SEe) are generated from an SE surface/cathode/"dynodes", when charged hadronic or electromagnetic particles (shower particles) penetrate an SE sampling module either placed between absorber materials $(\mathrm{Fe}, \mathrm{Cu}, \mathrm{Pb}, \mathrm{W}$ etc) in calorimeters, or as a homogeneous calorimeter consisting entirely of dynode sheets as the absorbers. An SE cathode - as on PMT dynodes - is a thin (10-50 nm thick) film. These films are typically simple metal-oxides $\mathrm{Al}_{2} \mathrm{O}_{3}, \mathrm{MgO}, \mathrm{CuO} / \mathrm{BeO}$, or other higher yield materials. The simple asfound native oxide on $\mathrm{Al}$ produces $6 \mathrm{SEe} /$ per incident at the peak of the SE yield parametrization as shown in Figs 2 below.

On the inner surface of a metal plate in vacuum, which serves as the entrance "window" to a compact vacuum vessel (metal or metal-ceramic), an SE film cathode is analogous to a photocathode, and the shower particles are similar to photons incident. The SEe produced from the top SE surface by the passage of shower particles, as well as the SEe produced from the passage of the shower particles through the dynodes, are similar to p.e. The SEe are then amplified by sheets of dynodes - metal-meshes or other planar dynodes (see Figs 2c, 3 below). The SEe yield $\delta$ is a strong function of momentum, following $\mathrm{dE} / \mathrm{dx}$ as in the Sternglass ${ }^{5}$ formulae. The yield follows a universal curve when normalized to the peak yield, as shown in Fig.1. As shown in Fig. 2 from CERN data, yields from a MIP on robust materials like alumina or titania films are only 1.10-1.2, requiring many dynodes for a MIP signal. On the other hand, as the shower is fully absorbed, those yields rise to about 6-7 at low energies. This variation with particle 
energy gives rise to quasi-compensation effects as the low energy nuclear fragments of hadron showers have high yields; for example $1 \mathrm{MeV}$ alpha particle produces $\sim 20 \mathrm{SEe}$.

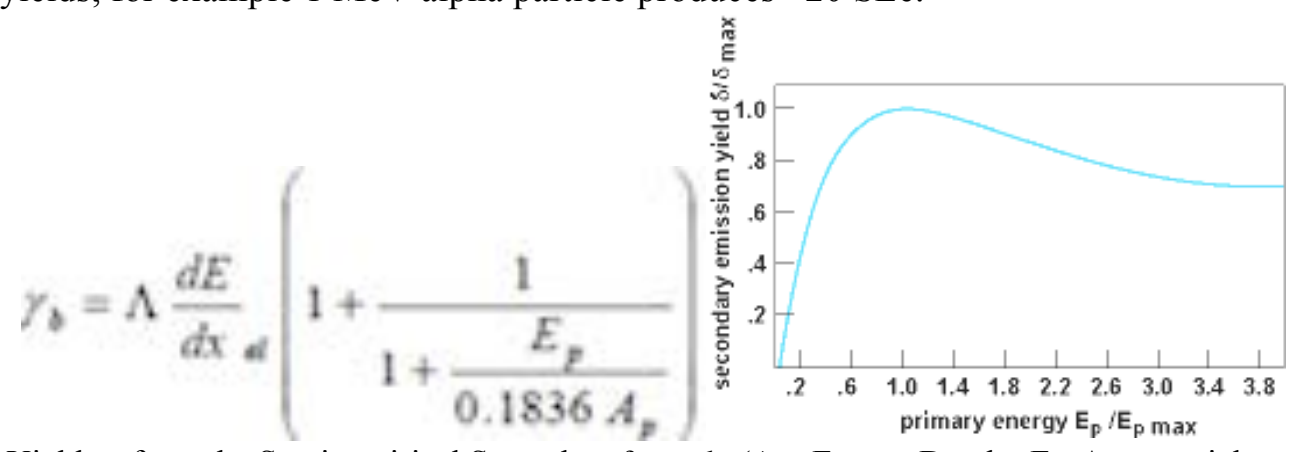

Figs. 1: SE Yield $\gamma_{\mathbf{b}}$ from the Semiempirical Sternglass formula ( $\Lambda=$ Escape Depth, $\mathrm{E}_{\mathrm{p}}, \mathrm{A}_{\mathrm{p}}=$ particle energy, mass) together with the universal curve of SE emission from metal oxides: the yield normalized by the maximum yield vs the particle energy normalized to the voltage/energy of the maximum yield emission.
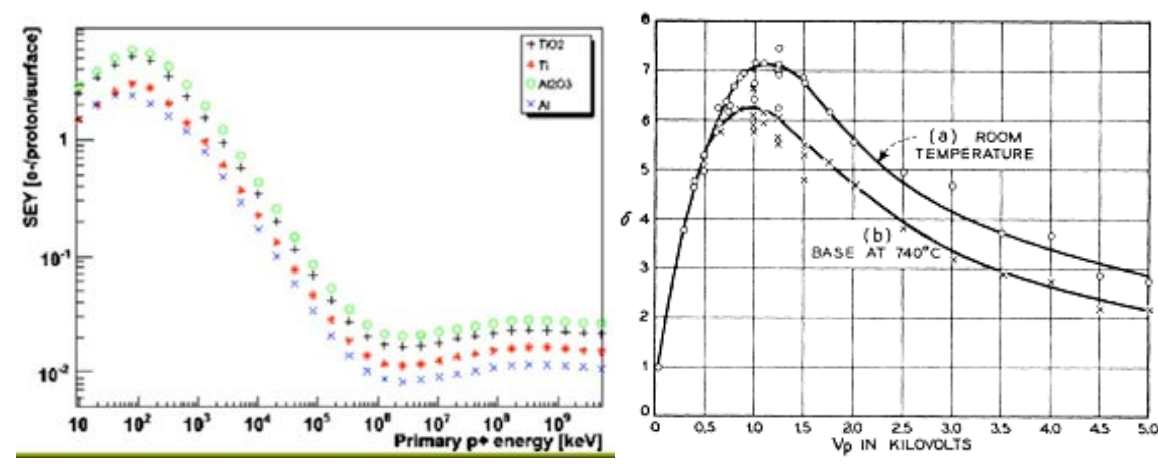

Fig 2a,b: Yield vs proton energy for titania and alumina and for the bare metals; Yield for $\mathrm{MgO}$ vs electron energy.

\section{Sensitivity of SEM detector}

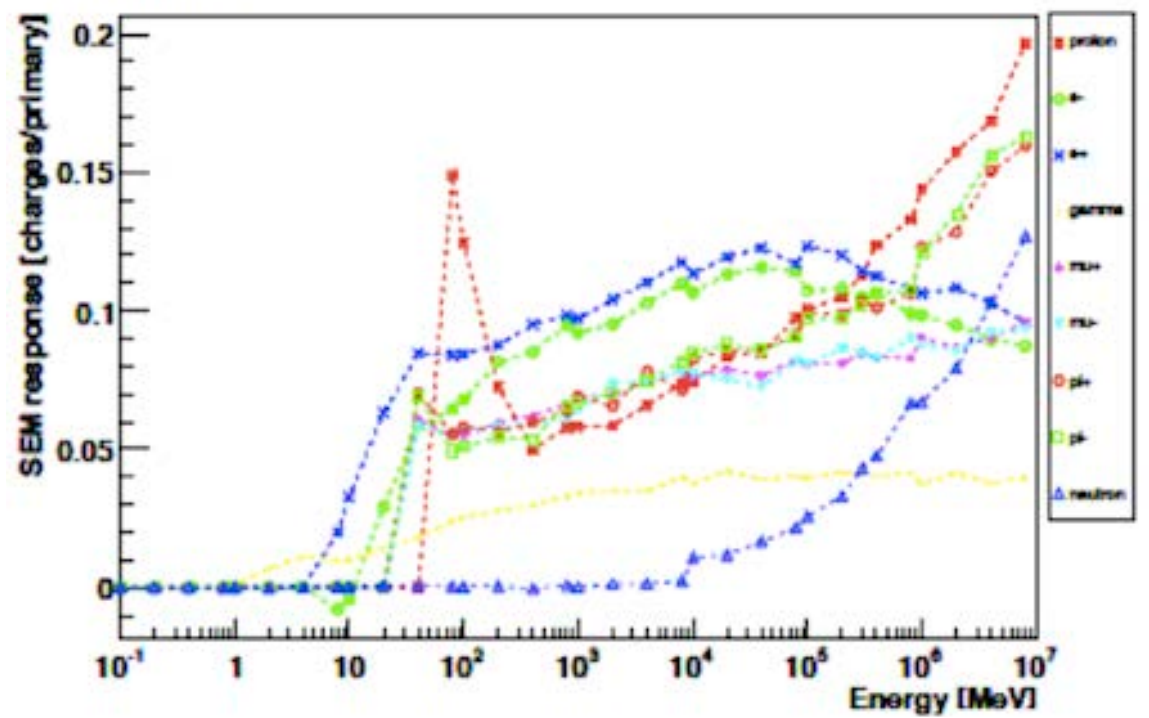

Fig. 2c: The number of SE electrons per primary collected in the CERN BLMS system test cell $(10 \mathrm{~nm}$ thick alumina SE surface) vs primary energy- $\mathrm{p}$ (red square), e-, e+( green-solid, blue-x), $\mu+, \mu$-(purple, magenta), pi + , pi(red-circle, green-open box), $\mathrm{n}$ (blue triangle), gamma(yellow) primaries. The higher yields above $\sim 10 \mathrm{GeV}$ are speculated to be from delta-rays. From $\operatorname{Ref}^{2}$ B. Dehning et al. ACHTUNG! The energy bins $<\sim 30 \mathrm{MeV}$ have a 
threshold from the thick metal entrance window - the regions below $1 \mathrm{MeV}$ have the highest yields; the negative signal at $\sim 8 \mathrm{MeV}$ is absorption of e- inside the signal electrode.

SE sensor modules can make use of electrochemically etched/machined or laser-cut metal mesh dynode sheets, as large as $\sim 30 \mathrm{~cm}$ square or more, to amplify the Secondary Emission Electrons (SEe), much like those that compact metal mesh or mesh dynode PMT's use to amplify p.e.'s. The secondary emission yield follows $\mathrm{dE} / \mathrm{dx}$ vs E or $\mathrm{p}$. MIPs typically saturate at $0.15 \mathrm{SEe} / \mathrm{mip}$, and rise by a factor of 20 at the low energy peak, typically 4-7 SE/incident particle, although thin film synthetic diamond can emit up to $120 \mathrm{SEe}$ at $3 \mathrm{KV}$ incident. We have studied this using GEANT4 ${ }^{6}$ to $\mathrm{MC}$ as below.
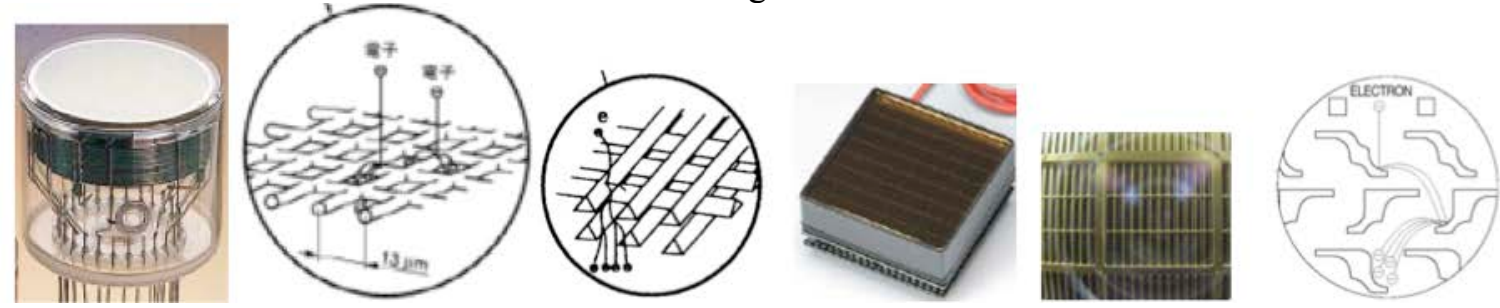

Figures 3a: Left to right top - mesh dynode PMT with typical meshes - note the $11 \mu \mathrm{m}$ scale of the mesh - and PMT using etched metal dynodes, as formed in sheets.

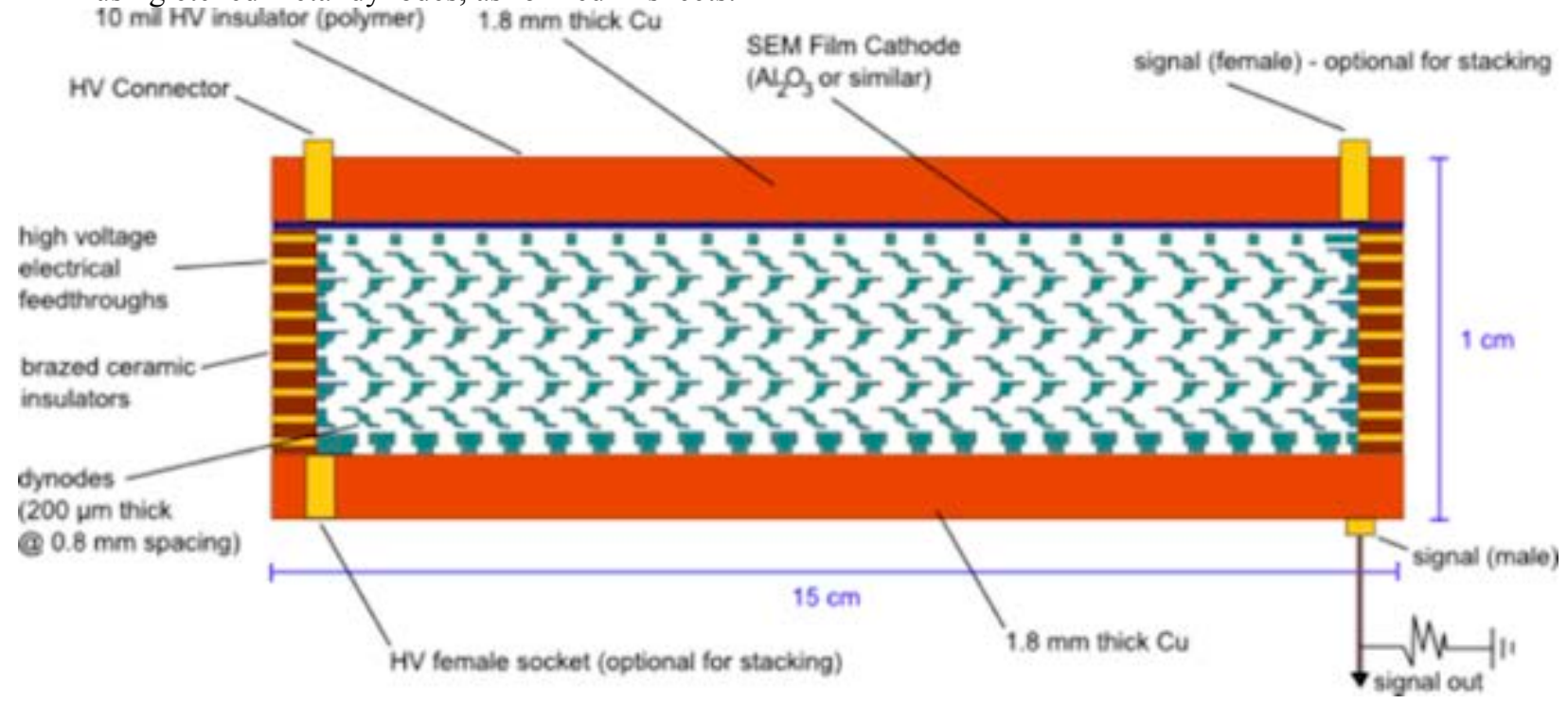

Figure 3b: A cartoon of a Secondary Emission calorimeter module, using etched metal sheet dynodes similar to those used by Hamamatsu. Commercial $\mathrm{Cu}-\mathrm{Be}$ meshes are also possible and low cost $\left(\$ 20 / \mathrm{m}^{2}\right.$ for appropriate fine metal woven sheets).
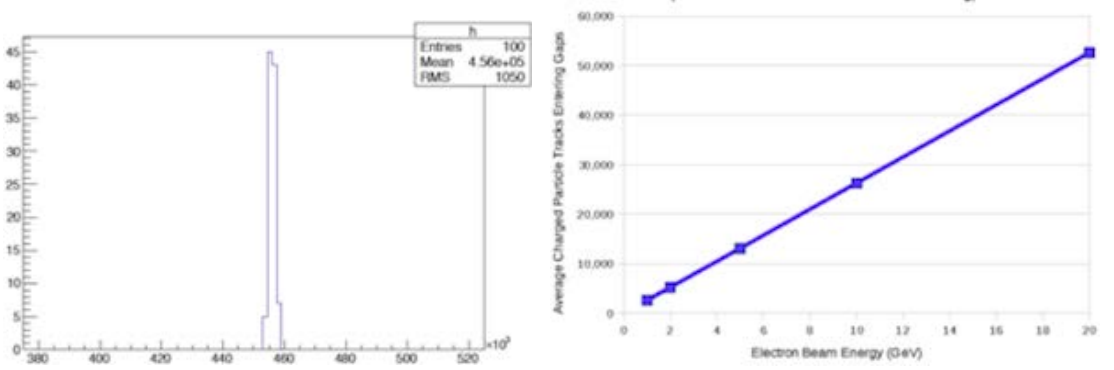

Figures 4: (Left) GEANT4-predicted histogram of the number of secondary emitted electrons by shower particles in an e-m showers initiated by $100 \mathrm{GeV}$ electrons (100 events), in a homogeneous mesh (no absorber plates) calorimeter consisting of $10 \mu \mathrm{m}$ wire $\mathrm{W}-\mathrm{mesh}, 30 \%$ transparent, spaced at $10 \mu \mathrm{m}$ calorimeter using the Sternglass formula for SEe yields, and assuming an SE yield for $100 \mathrm{eV}$ electrons of 
3. The stochastic term inferred is less than $1 \% / \sqrt{\mathrm{E}}(\mathrm{GeV})$ - (no systematics nor various noise issues are in the $\mathrm{MC}$ to date). The linearity (right) is outstanding. The density is about $40 \%$ of pure $\mathrm{W}$.

The construction requirements for an SE Sensor Module are much easier than a PMT, since:

1. the entire final assembly can be done in air;

2. there are no critical controlled thin film vacuum depositions for a photocathode, cesiation or other oxygen-excluded processes or other required vacuum activation is not necessary (although possibly desired for enhanced performance).

3. bake-out can be a refractory temperatures, unlike a photocathode which degrades at $\mathrm{T}>300^{\circ} \mathrm{C}$;

4. the SE module is sealed by normal vacuum techniques (welding, brazing, diffusion-bonding or other high temperature joinings), with a simple final heated vacuum pump-out and tip-off.

The modules envisioned are compact, high gain, high speed, exceptionally radiation damage resistant, rugged, and cost effective, and can be fabricated in arbitrary tileable shapes. Mesh dynodes will work at $10 \%$ gain in $1.2 \mathrm{~T}$ at $45^{\circ}$ to the B-field ${ }^{7}$. The SE sensor module anodes can be segmented transversely to sizes appropriate to reconstruct electromagnetic shower cores with high precision. The GEANT4 and existing calorimeter data estimated calorimeter response performance is between 35-50 Secondary Emission electrons (SEe) per $\mathrm{GeV}$ of electromagnetic deposition, in a calorimeter with $1 \mathrm{~cm}$ thick $\mathrm{Cu}$ absorbers interspersed with 15 planes of $\mathrm{Cu}$ mesh dynodes, with a gain per SEe of $\sim 10^{5}$ per SEe, and an $\mathrm{e} / \mathrm{pi}<1.2$. The calorimeter pulse width $10 \%-10 \%(90 \%)$ is estimated to be $<11 \mathrm{~ns}$ based on shower length, secondary electron transit time estimate, and mesh PMT TTS. A MC of a homogeneous (i.e. no absorber except dynode sheets) calorimeter using $10 \mu \mathrm{m} \mathrm{W}$ mesh sheets at $10 \mu \mathrm{m}$ spacing (about $40 \%$ of the density of $\mathrm{W}$ ) generates in excess of $40,000 \mathrm{SEe}$ per $\mathrm{GeV}$, and a stochastic term inferred of less than $1 \% / \sqrt{\mathrm{E}}(\mathrm{GeV})$, assuming each SEe is amplified by the downstream mesh to a conveniently detectable level $\left(\mathrm{g}>4 \times 10^{4}\right.$ per SEe). We emphasize that an SEe is treated exactly like a p.e. in a scintillator calorimeter. In a scintillator calorimeter, many photons are created, but typically $0.1-1 \%$ are collected and converted to p.e. by a PMT of SiPM; by contrast, in an SE calorimeter, relatively few SE electrons are created, but almost all are collected and amplified by the dynode stacks as SEe.

Initial Tests of SE Calorimetry: We have tested this concept at a CERN test beam in 2011 - using metal mesh PMT as shown in Fig 4 below. The photocathode was disabled by using a +HV base, operating the anode at $\sim+2 \mathrm{KV}, \mathrm{D} 1$ at ground, and the photocathode at small positive voltages. Blue LED showed that the photocathode was insensitized. The tube was operated with particles entering from the base side, with the photocathode window blackened. The tube was exposed to beams of $225 \mathrm{GeV}$ muons and $100 \mathrm{GeV}$ electrons entering a $3 \mathrm{~cm} \mathrm{~Pb}$ radiator, to simulate shower max.

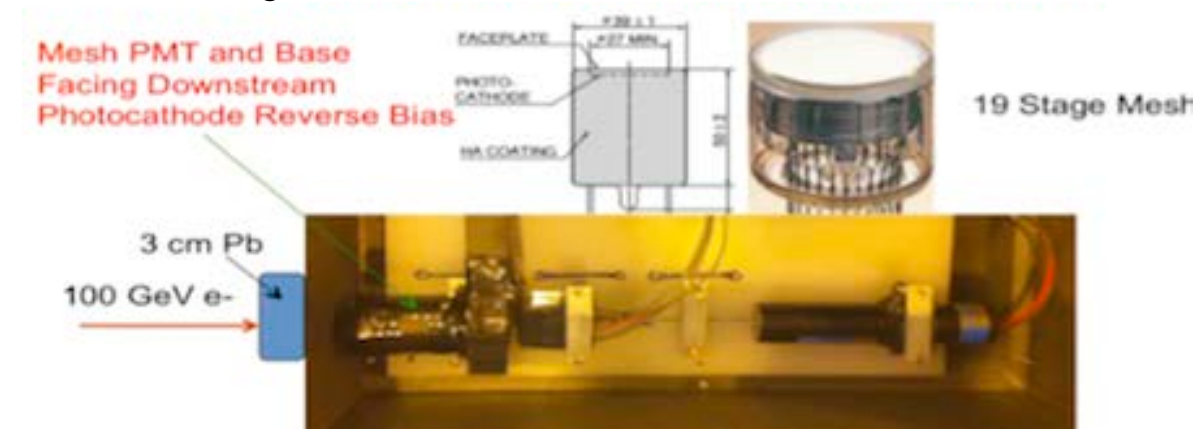

Fig 5: Beam Test Configuration for SE emission signals: Hamamatsu 19 stage mesh PMT in the CERN test beamline, in the phototube test box. $225 \mathrm{GeV} \mu+$ or $100 \mathrm{GeV} \mathrm{e}^{-}$hitting $3 \mathrm{~cm}$ of Pb radiator incident from left. The photocathode was disabled using a $+\mathrm{HV}$ base, anode at $+2.1 \mathrm{KV}$, Gain $=8 \times 10^{4}, \mathrm{D} 1=$ ground; Photocathode $=+10 \mathrm{~V}$ through $400 \mathrm{k} \Omega$ to ground. 

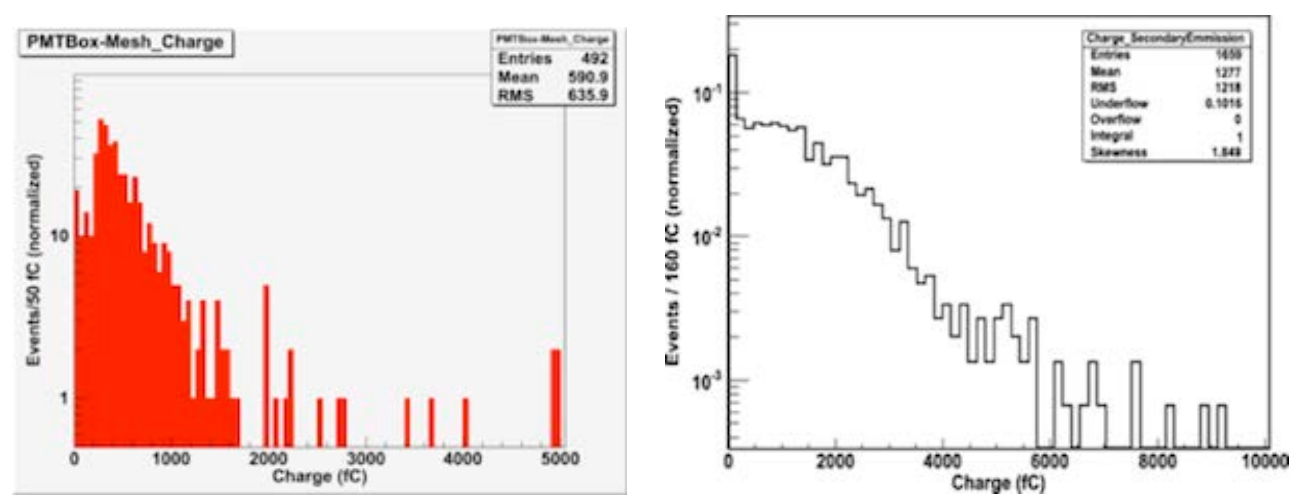

Fig 6(L): Beam test results of Mesh SEe "sensor": $100 \mathrm{GeV}$ electrons into $3 \mathrm{~cm} \mathrm{~Pb}$, with one SE sampling "cell". Peak in mesh PMT signal corresponds to 41 SE electrons (mesh gain $\sim 10^{5}$ ) at about shower max. The large width is essentially entirely consistent with shower width fluctuations on a single shower sampling after $3 \mathrm{~cm} \mathrm{~Pb}$. Fig 6(R): Detection efficiency - Muon Efficiency as selected by wire chambers; the peak at zero is below threshold indicating: $75 \%$ detection efficiency for a single particle through at most the first 5 meshes - after that, the gain falls below threshold. (Cu-Be Mesh PMT: 19 stages, g 2 x106; 1 st 5 mesh $\sim 80 \%$ effy at $20 \% /$ mesh). We infer that the yield on a MIP is 1.11 per SE surface in line with measurements from CERN as in Figs 2a, 2c.

The peak of the signal in the mesh PMT signal corresponds to $41 \mathrm{SE}$ electrons (mesh gain $\sim 10^{5}$ ) at about shower max. The large width is essentially consistent with shower fluctuations on a single shower sampling after $3 \mathrm{~cm} \mathrm{~Pb}$, over the 1.25" PMT face. This implies $>600 \mathrm{SEe} / \mathrm{GeV}$ is possible (i.e treated the same as $\sim 600 \mathrm{pe} / \mathrm{GeV}$ in a scintillator calorimeter) with a full calorimeter.

The detected muon signal was $74 \%$ efficient, consistent with 3 pe, consistent with MIP delta rays/SE electrons $>50 \mathrm{eV}$ from the photocathode surface, and SE from the 19 dynodes. The electron shower max was fully efficient, with a mean number of SEe of $\sim 30-40$, within a factor of 2 expected for a sample of a $100 \mathrm{GeV}$ shower at 5.5 Lrad and the dynode stack diameter. This implies with homogeneous mesh stack calorimeter (no other absorber) that $>600 \mathrm{SEe}$ per $\mathrm{GeV}$ are possible with the particular mesh used in the mesh PMT, consistent with the MC for this $\mathrm{Cu} / \mathrm{Be}$ mesh, and a resolution of $\sim 4 \% / \sqrt{\mathrm{E}}$.

Another test beam was run at CERN in 2012 using an array of metal mesh (19 stage) PMT with the Photocathode shut off, as assembled in groups of 9 into SE sensor modules. These modules are shown in the figure below. The SE sensor modules were interspersed with 1.75Xo Fe slabs as shown below. A variable length of Fe slabs (0-9Xo)were placed in front, and data taken with each thickness in front. The ADC histogram data with the MC ADC histogram of the set-up are shown below, and the MC wellmatches the ADC response of the SE sensor modules.

SE Module Beam Test
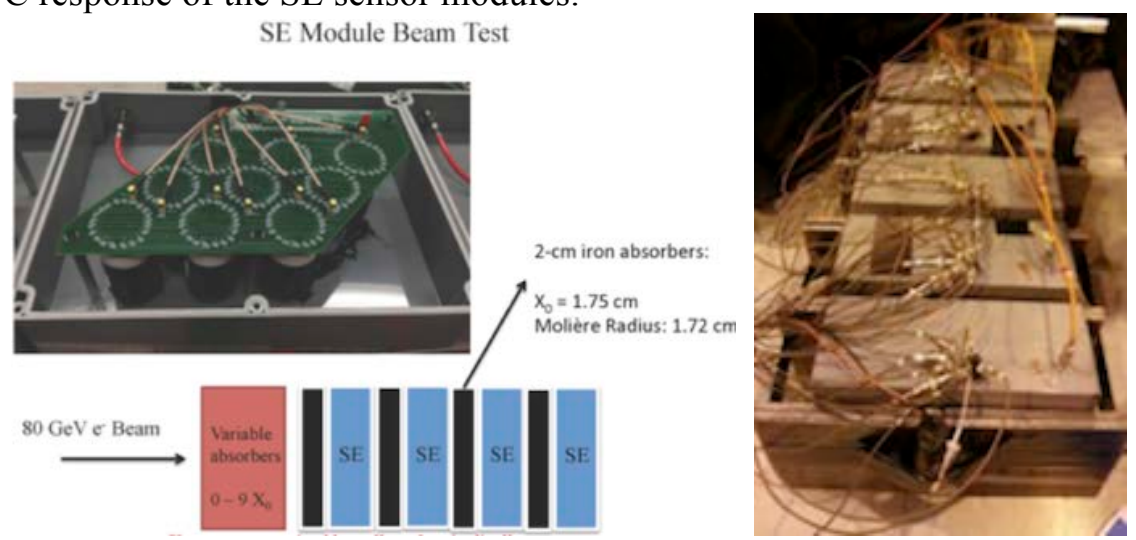

Figures 7: Left - an SE sensor module using 9 mesh dynode tubes with the photocathode shut off. Right: 5 sensor modules (as at left) stacked between 1.75Xo Fe absorbers. As shown above, data were taken with $80 \mathrm{GeV}$ electrons incident, varying the absorber thickness ahead of the modules, in order to simulate a full calorimeter. 
SE Module Simulation
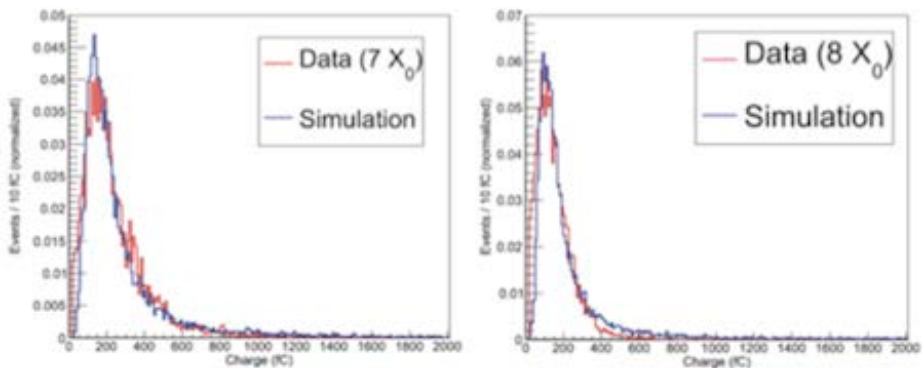

Figures 8: Data compared with MC of the ADC histogram response of the SE sensors to $80 \mathrm{GeV}$ electrons for showers after $7 \mathrm{Xo}$ and after $8 \mathrm{Xo}$ in front of the SEsensor stacks. Good agreement with the simulation.

We then used the MC to predict the energy resolution of a possible full calorimeter for electrons using this crude calorimeter $(1.75 \mathrm{Xo}$, with only the partial areal coverage per module of the showers as shown by the module construction above) sampling, and assuming enough modules to cover $30 \mathrm{Xo}$. These MC predictions are shown below.
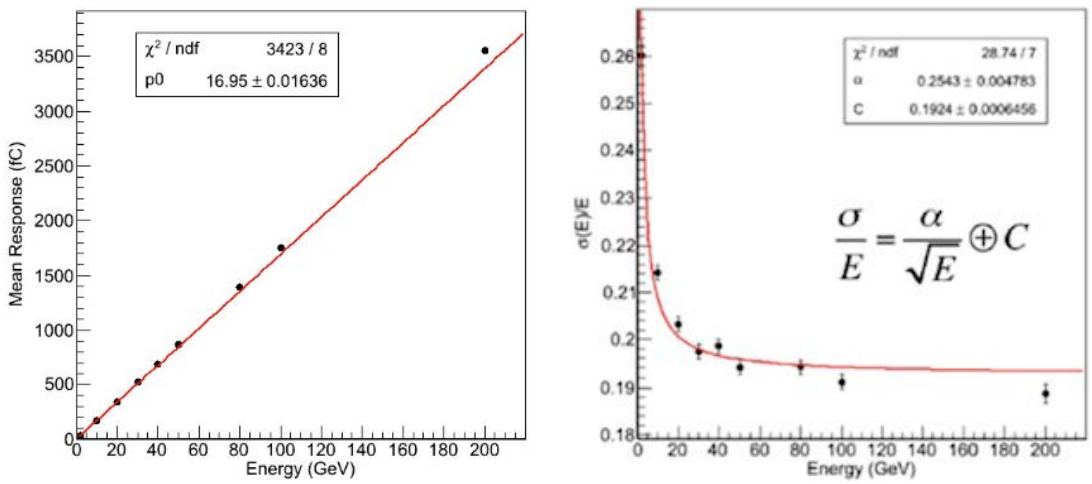

Fig. 9: Electron linearity and energy resolution in GEANT4 simulations of a 1.75 Xo FE sampling SEsensor calorimeter.

GEANT4 was tuned to simulation a "homogeneous" SE Calorimeter - a stack of W or Cu dynodes made of mesh, $10 \mu \mathrm{m}$ in thickness spaced at $10 \mu \mathrm{m}$ (about $40 \%$ of the density of $\mathrm{W}$ or $\mathrm{Cu}$ ), with no other absorber. The predicted resolution is $\sim 1-3 \% / \sqrt{ } \mathrm{E}$, assuming each $\mathrm{SEe}$ is treated like a p.e.
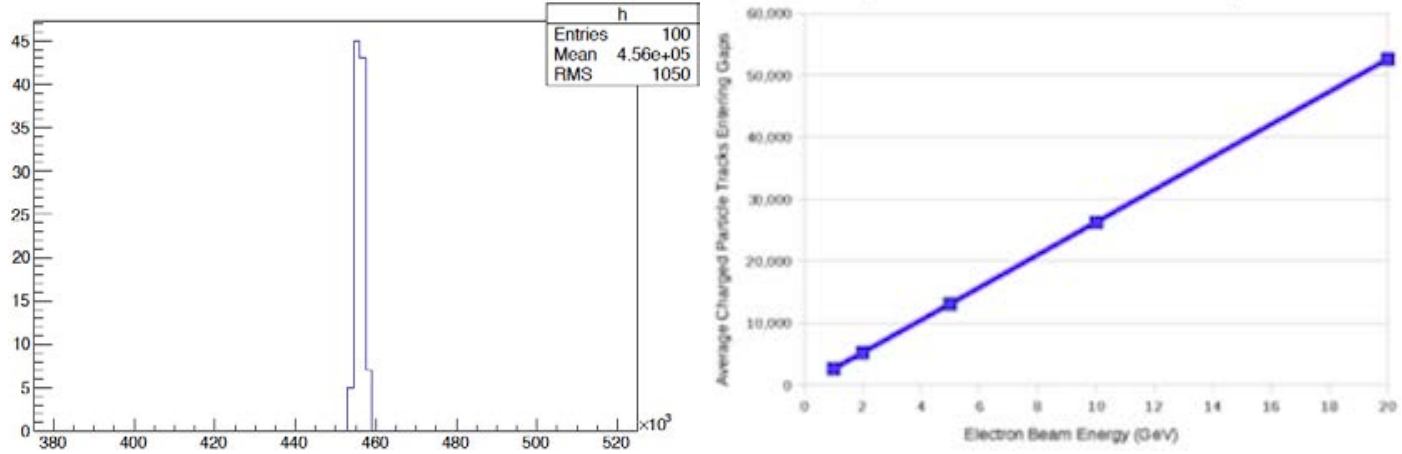

Figs. 10 (as 4 above): Left: GEANT4-predicted histogram of the number of secondary emitted electrons created by shower particles ( $\mathrm{e}^{-}$or $\mathrm{e}^{+}$) in e-m showers of $100 \mathrm{GeV}$ electrons (100 events), incident on a $10 \mu \mathrm{m} \mathrm{W}$-wire mesh spaced by $10 \mu \mathrm{m}$ - a homogeneous - no absorber plates - calorimeter, using the Sternglass formula for SEe yields on an oxide film with a peak yield of 3 at $100 \mathrm{~V}$. Right: GEANT4 predicted linearity on e-m showers for a homogeneous W-mesh calorimeter with $40 \%$ of the density of W. 


\section{Future Directions:}

Neutrons: Neutrons are an especially interesting application since the dynodes arranged as a dense stack can be individually coated on the back-side with a 1-10 micron thick $\mathrm{Li}^{6} \mathrm{~B}^{10}$ film. The film thickness would be adjusted to optimize alpha yield into vacuum from the absorption of neutrons in the $\mathrm{LiB}$, a tradeoff between thin so that the alpha resulting from neutron absorption escapes to cause secondary emission in neighboring dynodes, and thick to absorb neutrons. Alpha particles of 0.1-5 MeV have very high secondary yield $(\sim 20-80)$ as the specific ionization in the oxide films is very high $^{8}$. This has the potential for a unique neutron detector, and for HEP calorimetry where enhanced neutron detection may result in improved compensation.

High Secondary Yield Materials: We show classic high SE yield materials below ${ }^{9}$ (Fig.11). Synthetic diamond films have extraordinary secondary yields ${ }^{10}$, and are an obvious choice for higher performance, with a minimal number of dynode sheets(Fig 12).

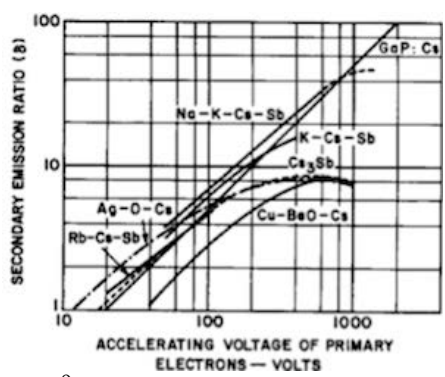

Fig. 11: Secondary yield of common materials 9 .
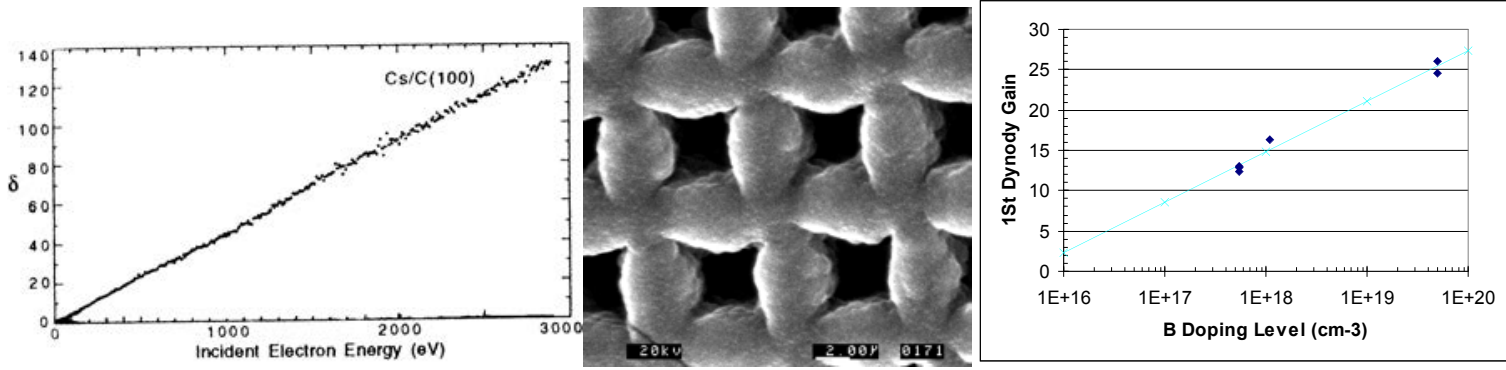

Figs 12: Synthetic Diamond SE yields: Left - Up to 130 at $3 \mathrm{KV}$ (data from Naval Research Labs); Middle - Mesh coated with $1 \mu \mathrm{m}$ of diamond. Center-center $=6 \mu \mathrm{m}$. Right - Diamond Dynode SE yield at $300 \mathrm{~V}$ vs B-doping level. $\sim 25$ per incident electron is easily available. (Data from NanoSciences, Oxford, CT and from Burle Electro-Optics)

Summary: The purpose of secondary emission calorimeters is particle (e, gamma, n, p, meson, nuclei) energy measurements in extreme radiation environments, and at very high rates. Benefits include fine segmentation, close packing and low cost. The modules envisioned are densely compact, high gain, high speed, exceptionally radiation damage resistant, rugged, and cost effective, and can be fabricated in arbitrary tileable shapes, to form large nearly hermetic detector arrays. The SE sensor module anodes can be segmented transversely to sizes appropriate to reconstruct incident gamma or neutron direction, with sufficient readout. Neutron detection may be enhanced by thin film coatings. Additional benefits outside of HEP include operation in harsh environments (space environs, high temperature/pressure environs e.g. boreholes $>1 \mathrm{~km}$ deep, high g-forces), imaging detectors in medicine and industry, radiocontraband detection, and beyond.

\section{REFERENCES:}

${ }^{1}$ R.G. Lye and A.J. Dekker, Theory of Secondary Emission, Phys. Rev. 107, 977-981 (1957) 
${ }^{2}$ B. Dehning et al., LHC BEAM LOSS DETECTOR DESIGN: SIMULATION AND MEASUREMENTS, FRPMN072 Proceedings of PAC07

${ }^{3}$ This is estimated from data in the Hamamatsu Photomultiplier Handbook, 1992.

${ }^{4} \mathrm{http}: / /$ www.colorado.edu/chemistry/chem5181/Lectures07/2007_MS4_Detectors_SNR.pdf

${ }^{5}$ Sternglass, EJ, Sci. Pap. 1772. Pittsburgh, PA: Westinghouse Research Laboratory, (1954).

${ }_{7}^{6} \mathrm{http}: / /$ geant4.cern.ch

${ }^{7}$ arXiv: 1207.4909

${ }^{8}$ J.N. Anno, Secondary Electron Production from Alpha Particles, J. Appl. Phys. 34, 3495 (1963)

${ }^{9}$ RCA PMT Handbook, 1978

${ }^{10}$ A.Shih et al., Secondary electron emission from diamond surfaces, J. Appl. Phys. 82, 1860 (1997) 


\title{
Snowmass 2013 White Paper \\ Quartz Capillary Cladding Anthracene and Polycyclic Aromatic Hydrocarbon(PAH)-Core Scintillating/WLS Fibers for High Rates and Radiation Damage Resistance
}

\author{
Fairfield University ${ }^{1}$ - University of Iowa ${ }^{2}$ - Argonne ${ }^{3}$ Collaboration \\ A.Albayrak-Yetkin ${ }^{2}$, B.Bilki ${ }^{2,3}$, J.Corso ${ }^{2}$, G.Jennings ${ }^{1}$, A.Mestvirisvilli ${ }^{2}$, Y.Onel ${ }^{2}$, I.Schmidt ${ }^{2}$, C.Sanzeni $^{1}$, \\ D.R.Winn ${ }^{1 *}$, T.Yetkin ${ }^{2}$ \\ *Contact/PI - winn@,fairfield.edu \\ cell/txt/vm 1+203.984.3993
}

\begin{abstract}
Quartz capillary tube/fibers have been filled with anthracene by a melt and vacuum inbibition process to fabricate a scintillating core fiber. Other polcyclic aromatic hydrocarbons(PAH)such as pTP, stilbene or Naphthalene are also well-suited to scintillating/shifting fiber cores. The resulting scintillating core with quartz cladding capillary fibers $(250-750 \mu \mathrm{m}$ cores) had a high specific light output when tested with muons ( 8 p.e. per MIP). These PAH core quartz capillary cladding scintillating/shifting optical fibers have the potential of high radiation resistance, fast response, and are applicable to many energy and intensity frontier experiments.
\end{abstract}

Introduction: Scintillating or wavelength-shifting (WLS) fibers ${ }^{1}$ suitable for fast and rad-hard detectors were constructed with the following precepts:

(1) PAH (polycyclic aromatic hydrocarbon) organic scintillators/WLS, such as anthracene, are rad-hard because of the strong bonds between benzene-rings, and the scintillation mechanism which de-excites a pair of excited molecules via vibrations (see the cartoon below for anthracene, as an example) ) $^{\mathbf{2} 3}$. The soft material in the solidified state is easily annealed, and not amenable to permanent color centers.

(2) Many PAH have decay constants $\sim 25 \mathrm{~ns}$ or less, matched to LHC (and beyond) interaction rates. These include pTerphenyl, stilbene, naphthalene and others.

(3)The peak light emission wavelengths are $\sim 380-520 \mathrm{~nm}$, good for long transmission \& PMT Q.E.

(4) The index of refraction is typically $\sim 1.62$, good for total internal reflection waveguiding with quartz $(\mathrm{n}=1.52)$ capillaries or tubes as a cladding (quartz is rad-hard). NA $=0.43$ for Anthracene/Quartz clad and the light trapping is $>3 \%$ of generated light.

(5) The melting points are modest, between $80^{\circ} \mathrm{C}-220^{\circ} \mathrm{C}$, but must be done under vacuum or inert atmosphere to prevent oxidation - in the present work we use a partial vacuum.

(6)The confining and impervious quartz-capillary:

a) forces the freezing liquid core to a continuous amorphous but nearly ordered solid fiber;

b) keeps the Gibbs free energy negative, for the reverse reaction to occur after any radiolysis; and

c) prevents interactions with $\mathrm{O}_{2}$.

These properties allow amorphous or microcrystalline soft-material based core fibers to be used for calorimetry or preradiators in high radiation environments. 
The meltable scintillating/shifting core materials include:

Anthracene:

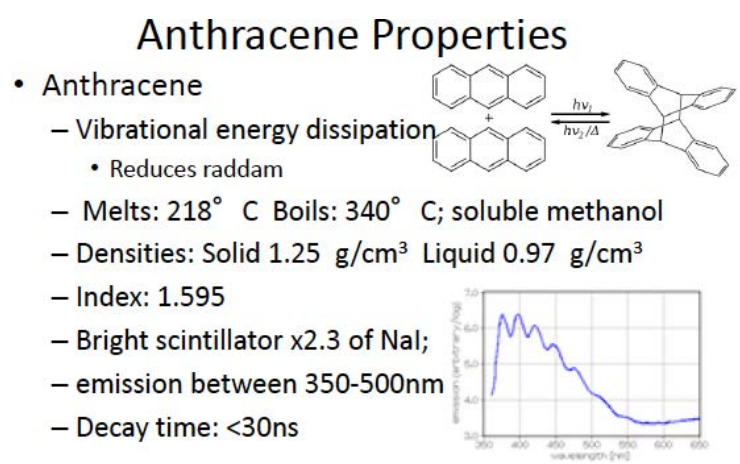

Stilbene(trans): $70 \%$ anthracene, $\tau_{\text {decay }} \sim 3.5 \mathrm{~ns}, \mathrm{n}=1.64, \lambda \sim 375-450 \mathrm{~nm}, \rho=1.22, \mathrm{~T}_{\mathrm{m}}=124^{\circ} \mathrm{C}$

pTerphenyl(pTP): $120 \%$ anthracene, $\tau_{\text {decay }} \sim 3.0 \mathrm{~ns}, \mathrm{n}=1.64, \lambda \sim 375-450 \mathrm{~nm}, \rho=1.22, \mathrm{~T}_{\mathrm{m}}=214^{\circ} \mathrm{C} \backslash \backslash \backslash \backslash \square$

Napthalene: rugged -2 benzene rings; $\tau_{\text {decay }} \sim 5 \mathrm{~ns} ; \mathrm{n} \sim 1.5 ; \lambda \sim$ "purple, $\mathrm{T}_{\mathrm{m}}=80^{\circ} \mathrm{C}$

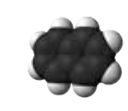

Figure 1: Properties of polycyclic aromatic hydrocarbons (PAH) suitable for quartz capillary scintillating cores.

Prototypes: Quartz capillaries were obtained from Polymicro ${ }^{4}$, with core diameters between 250-750 $\mu \mathrm{m}$, with an external low index doped quartz cladding. A vacuum tube furnace was assembled with temperature control as shown in Fig. 2 below. An inner pyrex vacuum burette with re-entrant parts for anthracene melt was used to hold the capillaries, with one end resting in anthracene powder. The partial vacuum was $\sim 2-3 \times 10^{-3}$ Bar. We recommend better vacuum, and prefilling with nitrogen or Argon. After a temperature threshold was crossed, typically about $5-8^{\circ} \mathrm{C}$ past the melting point, the liquid anthracene uniformly filled the fibers, even energetically fountaining from the top, presumably due to capillary action. More elevated temperatures caused the anthracene to evacuate the fibers, or to begin to oxidize. The temperature was brought down over $\sim 30$ minutes. Optimization of the process requires further work. Examination of the resulting fibers with a microscope showed uniform cores with essentially no inclusions, bubbles, or defects in most fibers.

Test Results: Strong scintillation was observed with a UV light on one end, with the scintillation light shining from the far ends (Figs. 4). A ${ }^{137}$ Cs source also evidenced scintillation detected by a PMT. The fibers were then mounted as a close-packed 2 layer ribbon in an Al holder, and read on each end by an $8 \mathrm{~mm}$ diameter Hamamatsu metal envelope PMT. They were exposed to a muon beam in the CERN H2 test beam, as defined by a wire hodoscope and muon trigger. The ADC spectra are shown in the Figures below. Remarkably, $\sim 9-10$ p.e. per end was measured as shown in Figs. 5,6; this rivals or exceeds existing plastic scintillating fibers, and was consistent with estimates (see the figures). The fibers were too short $(\sim 25 \mathrm{~cm})$ to observe attenuation effects. 


\section{Rig: Vacuum Tube Furnace - Anthracene "Double Boiler"}

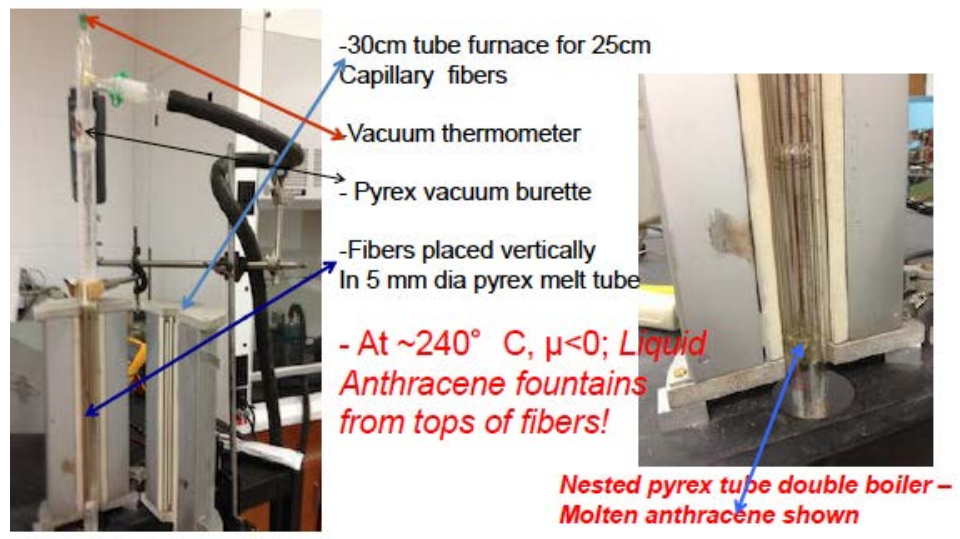

Fig. 2: The vacuum tube furnace used to fill quartz capillaries with anthracene cores. The capillary action at $\sim 240^{\circ} \mathrm{C}$ fountains the molten anthracene out from the tops of the quartz capillaries, and then a tapered cooling cycle forms perfect scintillating core fibers in the capillaries.

\section{$25 \mathrm{~cm}$ Anthracene Core Quartz Capillary Fibers}

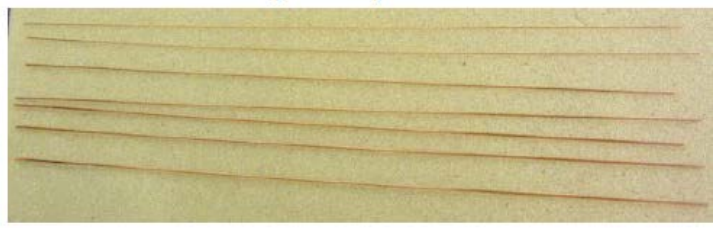

\section{$\mathrm{NA}=0.43$}

Core Diameters: $250-750 \mu \mathrm{m}$

Fig. 3: Fabricated anthracene core fibers with quartz capillary claddings.

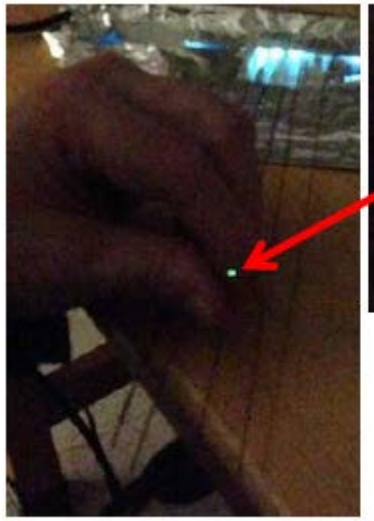

7 fibers pumped by $\mathrm{Hg}$ UV Pen Source, With one fiber bent up to camera
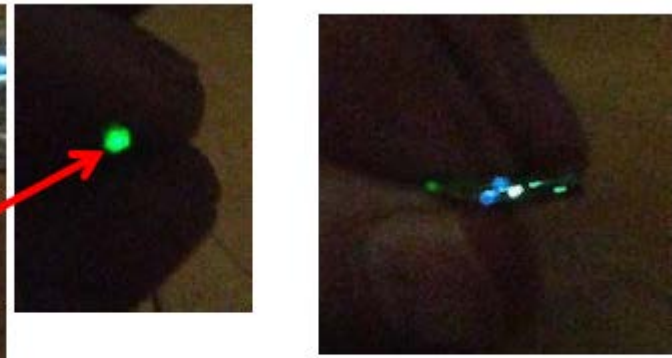

Fibers bent toward camera

Fibers cores: $250 \mu \mathrm{m}-750 \mu \mathrm{m}$

Fig. 4: Preliminary tests of the WLS and scintillation properties of the anthracene core quartz capillary optical fibers, using a UV light source to pump one end of the fibers, the photographs show the far end scintillation. ${ }^{137} \mathrm{Cs}$ sources also showed scintillation, but were too weak to photograph; a PMT was integrated with a fA meter. The fibers were inspected with a microscope for obvious core defects. 


\section{Expected Anthracene Fiber Pulse: $\sim 200 \mathrm{KeV} / \mathrm{mm} \times 0.25 \mathrm{~mm} \times 40$ photons $/ \mathrm{KeV}$ x $2 \%$ transmission $\times 20 \%$ QE 8 p.e. Typical Observed Pulse: \\ $\sim 8-9$ p.e.}

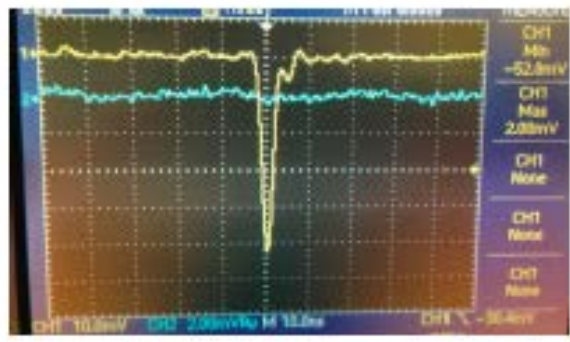

Typical pulse in $80 \mathrm{GeV}$ e- beam

Fig.5: Typical pulses from one end of the anthracene fibers exposed to MIPS. Pulse width $10 \%-10 \%<10 \mathrm{~ns}$.

Both charges $>20 \mathrm{fC}$

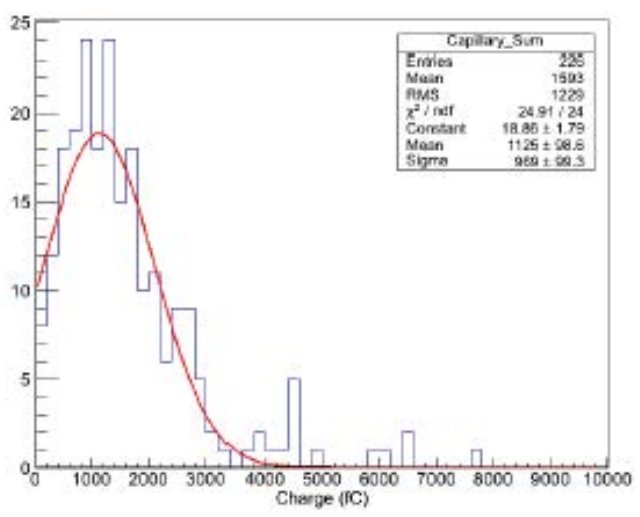

Fig. 6: The electron beam of $2 \times 2 \mathrm{~cm}$ was perpendicular to the fiber 2 layer ribbon (fibers as in Fig. 3), which were arranged as shown at left, and read out from both fiber ends with $8 \mathrm{~mm}$ diameter Hamamatsu PMT. The RH figure shows the ADC distribution from the sum of both fiber ends, which corresponds to a mean of about 18-20 p.e. or $\sim 9$-10p.e. per end. (The threshold cut of $20 \mathrm{fC}$ per end corresponds to $\sim 1 / 4$ p.e. per end.)

\section{Summary: Anthracene Core Quartz-Cladding Fibers:}

These novel fibers show great promise, with a high light output, exceeding many existing fibers. Future work is planned to include radiation damage studies, the fabrication of longer fibers, and attenuation length measurements. We request support for continuing this development.

\section{REFERENCES:}

${ }^{1}$ For a review, see H.Leutz, Scintillating Fibers, NIM, Vol 364, 422 (1995)

${ }^{2}$ H B Clarke et al 1962 The Scintillation Phenomenon in Anthracene I. Radiation Damage Proc. Phys. Soc. 79366 doi:10.1088/0370-1328/79/2/315

${ }^{3}$ GA Simpson et al., Radiation Induced Paramagnetic Centers in Anthracene. Molecular Crystals, Vol. 5165 (1968)

${ }^{4} \mathrm{http}$ ://www.molex.com/molex/products/group?key=polymicro\&channel=products 


\section{Micro-Pattern Gas Detectors for Calorimetry}

J. Yu (UTA), M. Hohlmann (Florida Inst. of Tech.), V. Polychronakos (BNL) and A. White

Physics Driver: All physics requiring precision energy measurements and triggering in future high-energy lepton colliders (ILC, CLIC, muon collider, etc).

Time Frame: Long

Physics Justification: Precise determination of the properties of the Higgs and any other newly discovered particles at the $\mathrm{LHC}$ require unprecedented jet energy resolution of $3 \% \Delta \mathrm{E} / \sqrt{\mathrm{E}}$ so that the invariant masses of $\mathrm{W}$ and $\mathrm{Z}$ particle in their two jet final states can be distinguished. A good technique for meeting this requirement is the particle flow algorithm (PFA) which utilizes the momentum measured in the tracking system to replace the charged particle energy deposit in the calorimeter. The calorimeter measures the energy deposit from the neutral hadrons and any electromagnetic particles resulting from secondary interactions. PFA is already in use in the CMS experiment to improve the experiment's jet energy resolution.

Technical Requirements: An important technical requirement for a successful PFA is the ability to match and subtract the charged particle energy deposit and to minimize confusion. A PFA requires a fine-granularity calorimeter for tracking individual shower particles. The calorimeter cell size must be optimized to meet this requirement. Micro-pattern gas detectors (MPGDs) are a promising candidate technology for such a tracking and imaging calorimeter. Economic construction of large-area MPGD detectors, low-cost electronic readout, highest tolerance against radiation damage, high-rate capability of $\mathscr{\odot}\left(100 \mathrm{MHz} / \mathrm{cm}^{2}\right)$ are some of the technical requirements. Suitable low-cost readout electronics are necessary to accommodate the large number of channels.

Current Technical Capabilities: A prototype RPC based calorimeter with one-bit digital readout electronics system was constructed and has demonstrated feasibility of an imaging calorimeter. Tracking detectors based on MPGD's such as Gas Electron Multipliers and Micromegas are operating with good stability in current experiments at particle rates up to 12 $\mathrm{MHz} / \mathrm{cm}^{2}$ (TOTEM). Single-hit efficiencies of typically $98 \%$ or better have been seen in current experiments and various beam test experiments. Best track position resolution and timing resolution achieved are on the order of $50 \mu \mathrm{m}$ (COMPASS) and 3-4 ns (LHCb). The flexibility of MPGD's allows various shapes of detectors. MPGD's can be made thin, typically $1 \mathrm{~cm}$ or less. Anode patterns take many forms from simple micro-strips to complex two-dimensional patterns. Advanced MPGDs are now reaching sizes of 1-2m length at the prototype level (ATLAS and CMS). On-board electronics for rapid pre-amplification are being developed.

\section{Key R\&D Directions:}

- Optimal structures and sizes of the readout anode for precisely matching tracks to energy deposition

- Cost effective on-board readout electronics and number of readout digitization bits

- Innovative signal induction structures to reduce readout cost and to improve performance

- Structures with resistive layers (e.g. Thick-GEM or Glass GEM) for discharge control 
- Integration of flex-circuit readout electronics directly into the MPGD structure

- Detector structures that minimize dead area

- Development of large-scale MPGD base material (GEM foils and Micromegas) construction techniques for (industrial) mass production

- Development of an industry base with MPGD mass production capabilities within the US 